\title{
Impact of the Object Constraint Language in UML-based Development
}

By

Han Daphne Yan-Bondoc

A thesis submitted to the Faculty of Graduate Studies and Research in partial fulfillment of the requirements of the degree of

Master of Computer Science

Ottawa-Carleton Institute of Computer Science

School of Computer Science

Carleton University

Ottawa, Ontario, K1S 5B6

Canada

May 2004

Copyright (C) 2004 by Han Daphne Yan-Bondoc 


\author{
National Library \\ of Canada \\ Acquisitions and \\ Bibliographic Services \\ 395 Wellington Street \\ Ottawa ON K1A ON4 \\ Canada
}

Bibliothèque nationale

du Canada

Acquisisitons et services bibliographiques

395 , rue Wellington Ottawa ON K1A ON4 Canada
Your file Votre référence ISBN: 0-612-93963-4

Ourfile Notre référence ISBN: 0-612-93963-4
The author has granted a nonexclusive licence allowing the National Library of Canada to reproduce, loan, distribute or sell copies of this thesis in microform, paper or electronic formats.

The author retains ownership of the copyright in this thesis. Neither the thesis nor substantial extracts from it may be printed or otherwise reproduced without the author's permission.
L'auteur a accordé une licence non exclusive permettant à la Bibliothèque nationale du Canada de reproduire, prêter, distribuer ou vendre des copies de cette thèse sous la forme de microfiche/film, de reproduction sur papier ou sur format électronique.

L'auteur conserve la propriété du droit d'auteur qui protège cette thèse. $\mathrm{Ni}$ la thèse ni des extraits substantiels de celle-ci ne doivent être imprimés ou aturement reproduits sans son autorisation.
In compliance with the Canadian Privacy Act some supporting forms may have been removed from this dissertation.

While these forms may be included in the document page count, their removal does not represent any loss of content from the dissertation.
Conformément à la loi canadienne sur la protection de la vie privée, quelques formulaires secondaires ont été enlevés de ce manuscrit.

Bien que ces formulaires aient inclus dans la pagination, il n'y aura aucun contenu manquant. 
PAGINATION ERROR.

TEXI COMPLETE.
ERREOR DE PAGINATION.

LE TEXTE EST COMPLET. 


\begin{abstract}
The Object Constraint Language (OCL) was introduced as part of the Unified Modeling Language (UML). Its main purpose is to make UML models more precise and unambiguous by providing a constraint language describing constraints that the UML model alone does not convey, including operation contracts and statechart guard conditions. There is an ongoing debate regarding the usefulness of using OCL in UMLbased development, questioning whether the additional effort and formality is worth the benefit. It is argued that natural language may be sufficient, and using OCL may not bring any tangible benefits. This thesis presents the results of a controlled experiment investigating the impact of using OCL on defect detection, comprehension and maintenance of UML analysis documents. The results show that, under certain conditions, significant benefits can be obtained by using OCL in combination with UML analysis.
\end{abstract}




\section{Acknowledgements}

Many people have contributed their helpful assistance to the completion of this thesis. This thesis would have never been possible without them. I would like to first thank Dr. Lionel Briand and Dr. Yvan Labiche for their greatly appreciated guidance and encouragement.

My husband Nick for his editorial help, his inspirational and moral support.

Last but certainly not least, I would like to show my gratitude to my mother Hong Yan, my grandparents Zhaonong Wang and Haojing Yan, who have been generous with their encouragement throughout. 


\section{Table of Contents}

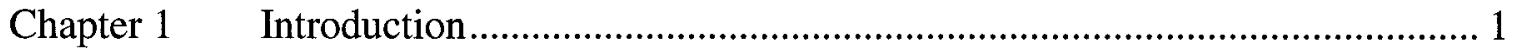

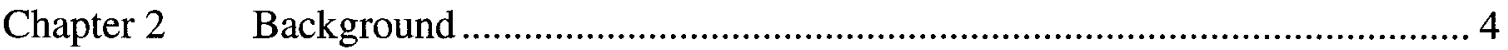

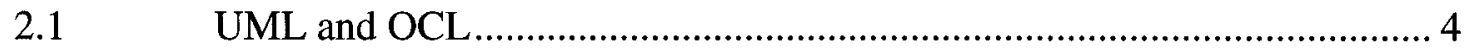

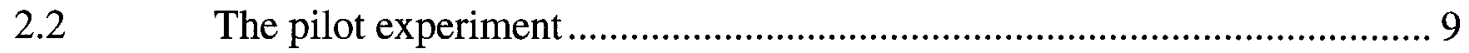

2.3 Experimental design terminology and principles ............................... 12

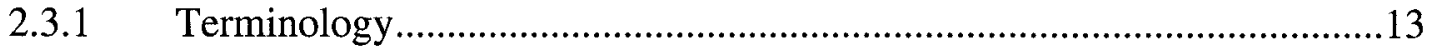

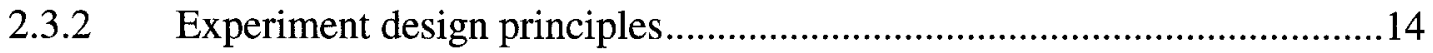

Overview of the statistics tests used …................................................... 16

2.4.1 Student's t-test, Mann-Whitney-Wilcoxon test, and Paired t-test .............16

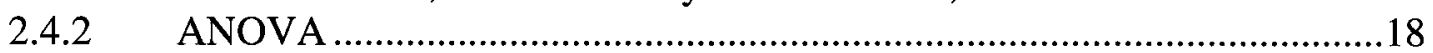

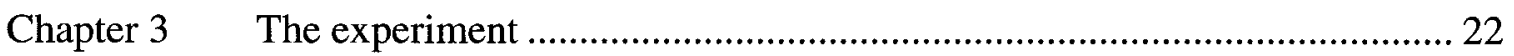

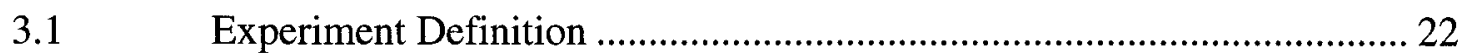

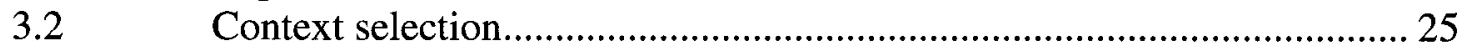

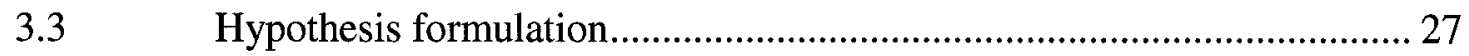

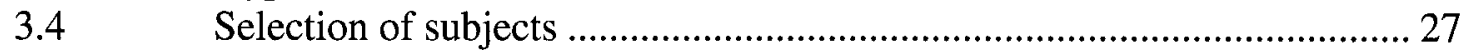

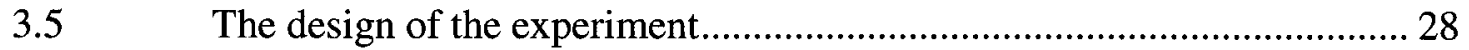

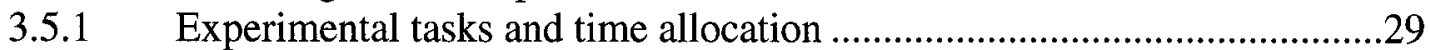

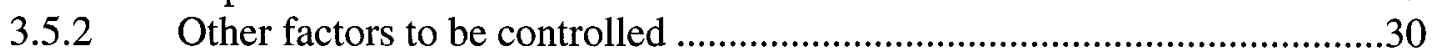

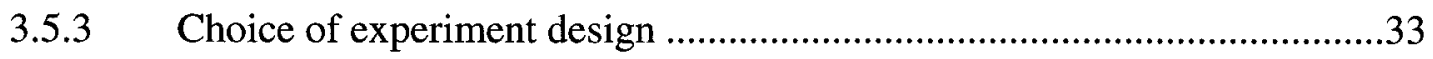

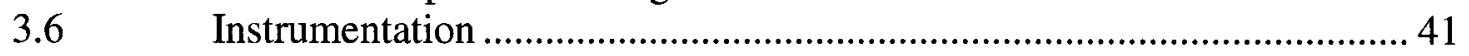

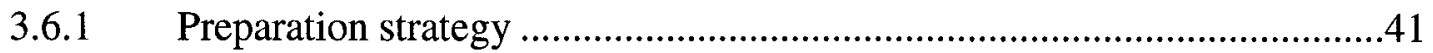

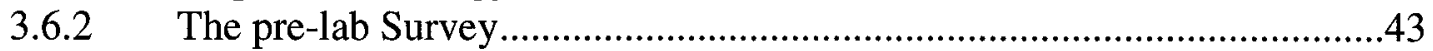

3.6.3 The UML system analysis documents .................................................43

3.6.4 Documents with seeded defect.............................................................44

3.6.5 Questionnaires for the Comprehension and the Maintenance tasks ...........46

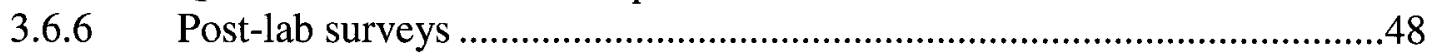

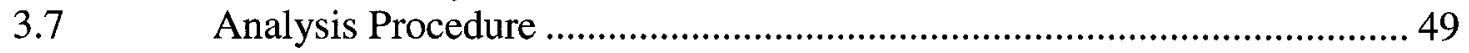

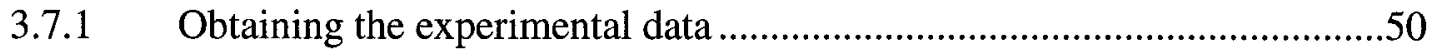

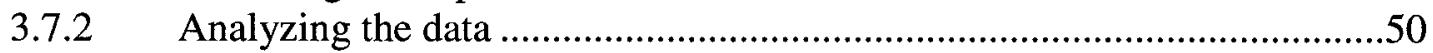

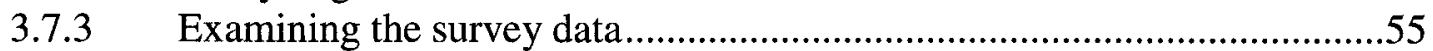

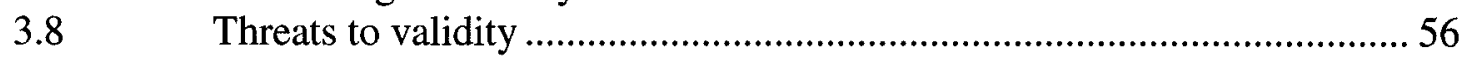

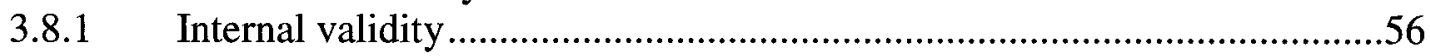

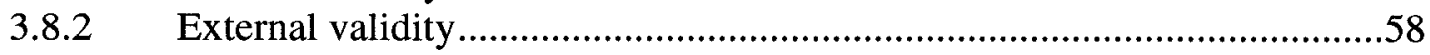

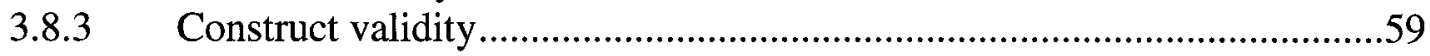

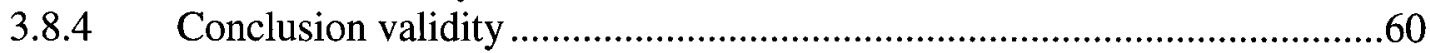

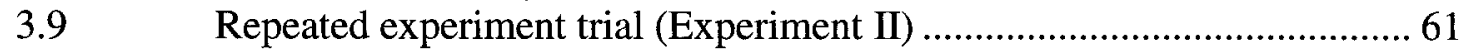

3.9.1 Differences in Experiment II ...........................................................61

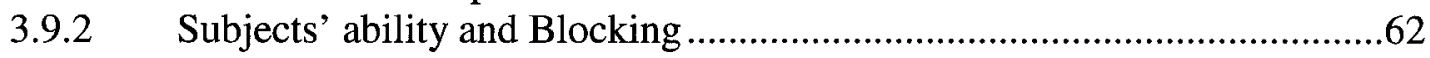




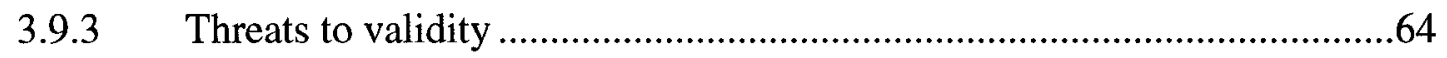

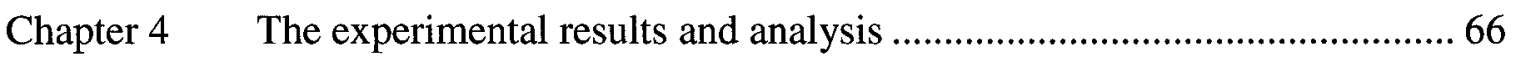

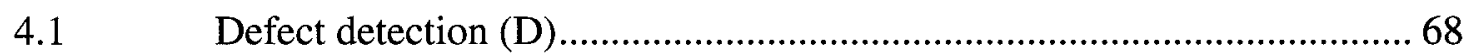

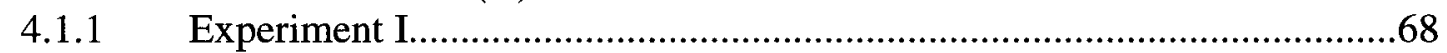

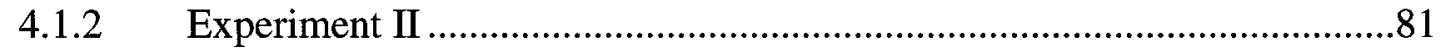

4.1.3 Experiment I and Experiment II ........................................................91

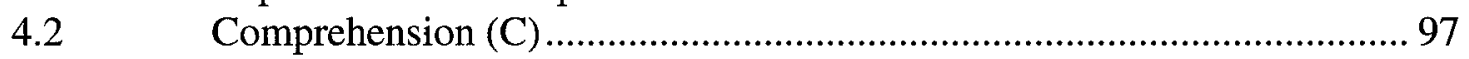

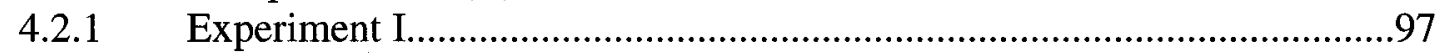

4.2.2 Experiment II ......................................................................................107

4.2.3 Experiment I and Experiment II ..........................................................115

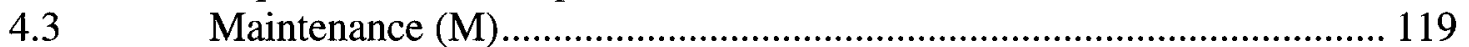

4.3.1 Experiment I............................................................................. 119

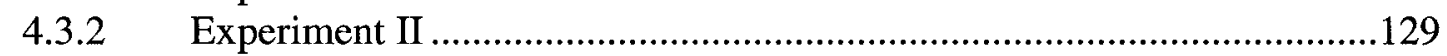

4.3.3 Experiment I and Experiment II ........................................................138

$4.4 \quad$ Discussion of results ....................................................................... 142

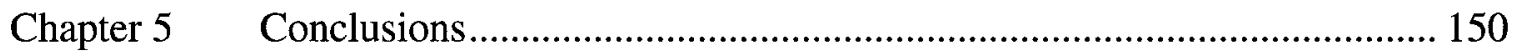

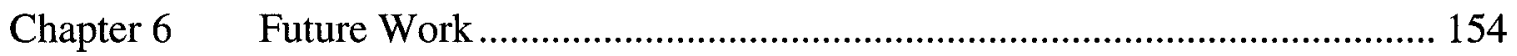

6.1 Issues encountered in the experiment ………………………............... 154

6.2 Considerations for future experiments .................................................... 155

$6.3 \quad$ Other aspects of interest.................................................................... 156

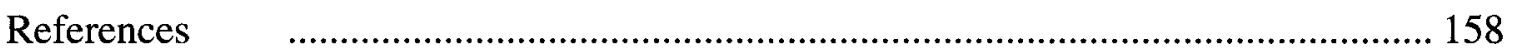

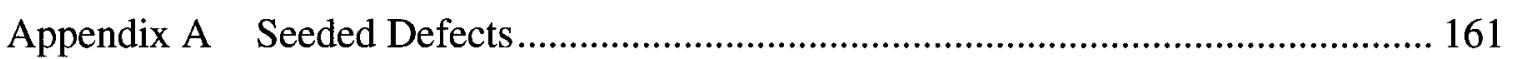

Appendix B Questionnaires............................................................................... 164

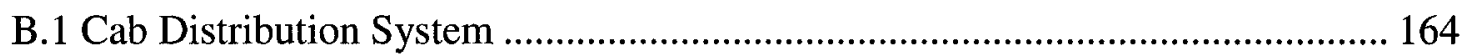

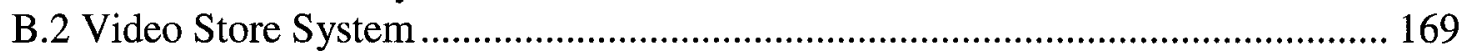

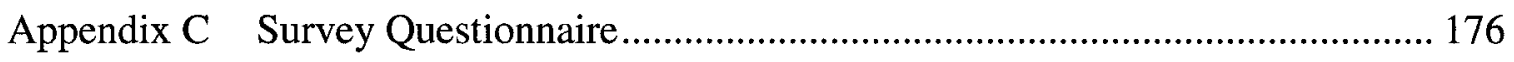

C.1 Pre-lab Survey Questionnaire............................................................................ 176

C.2 Defect Detection Survey Questionnaire ........................................................... 177

D.3 Comprehension and Maintenance Survey Questionnaire .................................... 177

Appendix D Survey Results ............................................................................. 179

Appendix E Assessing equal variance ............................................................. 192 


\section{List of Figures}

Figure 1. An example of UML operation specification ........................................... 5

Figure 2. Pre- / Post-condition of ReservationControl::cancelReservation in OCL......... 7

Figure 3. Example defects seeded in the VS system analysis document...................... 45

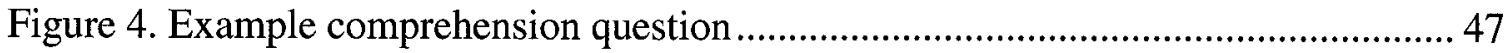

Figure 5. Example Maintenance task question .................................................. 47

Figure 6. A survey questionnaire example .................................................... 49

Figure 7. Graph of means by Method and Ability (example) ........................................ 55

Figure 8. Grade distribution of Experiment I...................................................... 58

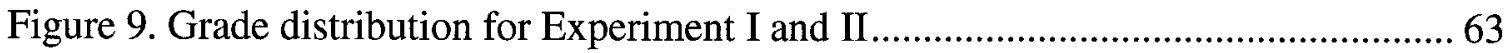

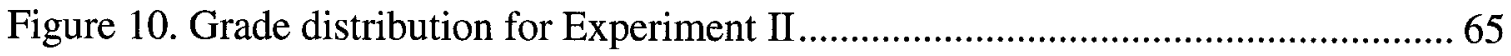

Figure 11. Graph of means by Method and Ability - D-1-I .................................... 73

Figure 12. Graph of Means by Method and System - D-1-I ...................................... 74

Figure 13. Graph of means (Method \& Ability) - D-3-I .......................................... 76

Figure 14. Graph of Means (Method \& System) - D-3-I ......................................... 78

Figure 15. Graph of Means by Method and Ability - D-1-II .................................... 85

Figure 16. Graph of Means by Method and System - D-1-II...................................... 86

Figure 17. Graph of Means by Method and Ability - D-3-II ...................................... 89

Figure 18. Graph of Means by Method and System - D-3-II....................................... 89

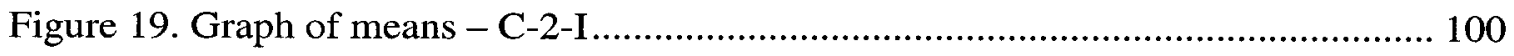

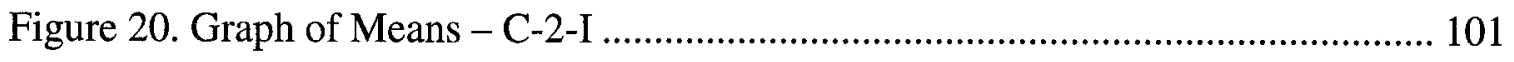

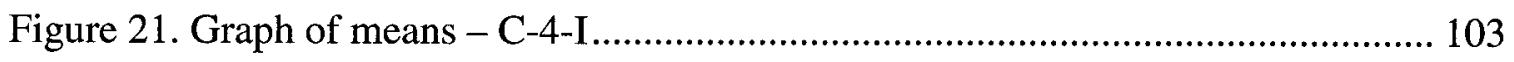




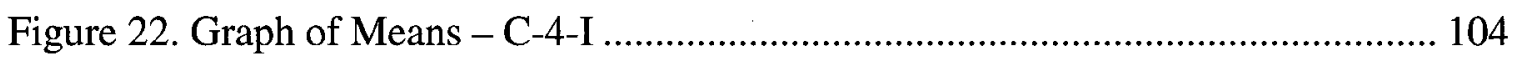

Figure 23. Graph of Means by Method and Ability - C-2-II .................................... 110

Figure 24. Graph of Means by Method and System - C-2-II ..................................... 111

Figure 25. Graph of Means by Method and Ability - C-4-II ...................................... 113

Figure 26. Graph of Means by Method and System - C-4-II ................................... 113

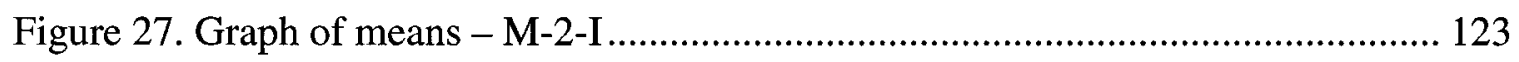

Figure 28. Graph of Means - M-2-I.................................................................. 124

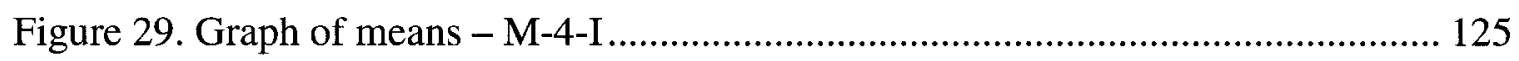

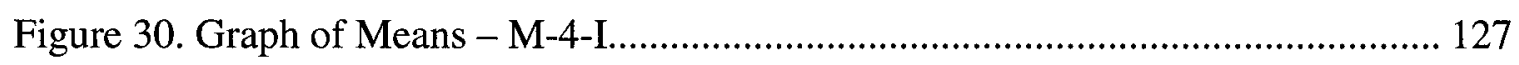

Figure 31. Graph of Means by Method and Ability - M-2-II................................... 133

Figure 32. Graph of Means by Method and System - M-2-II .................................... 133

Figure 33. Graph of Means by Method and Ability - M-4-II....................................... 136

Figure 34. Graph of Means by Method and System - M-4-II ................................... 136

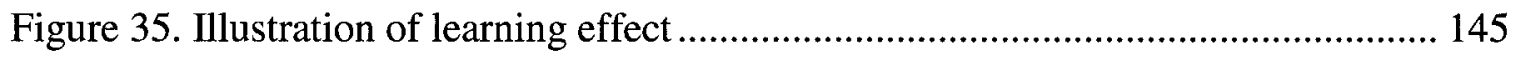




\section{List of Tables}

Table 1. Design of the pilot experiment.................................................................... 10

Table 2. A typical $t$-test/Wilcoxon test table ........................................................... 18

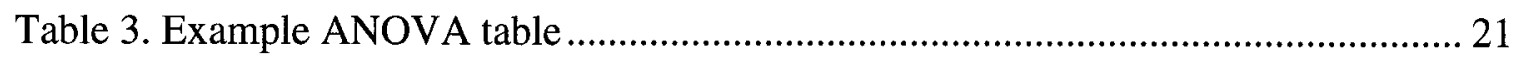

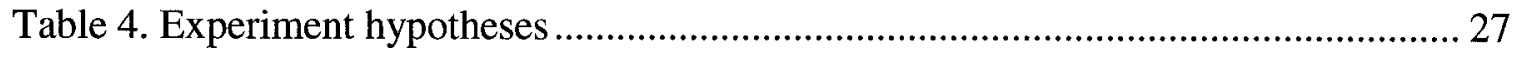

Table 5 Preliminary one-factor experiment design with repeated measurements ........... 34

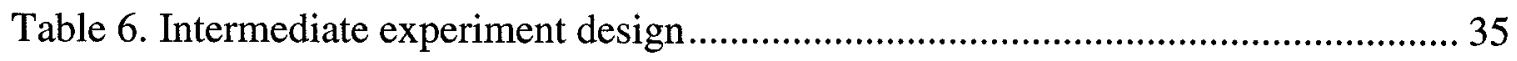

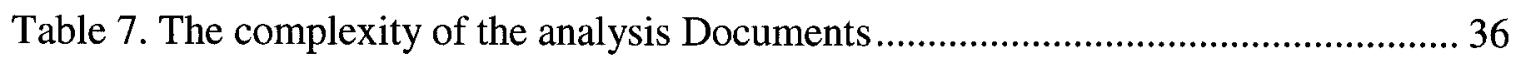

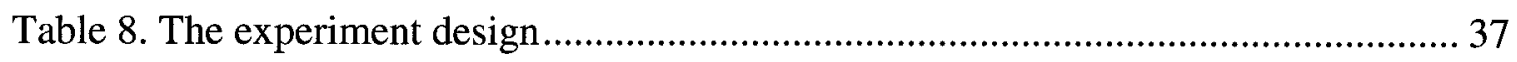

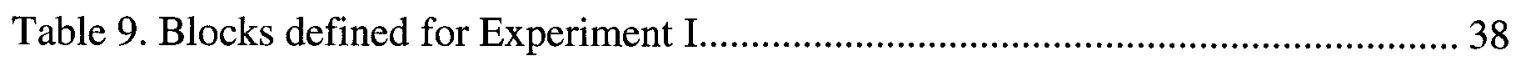

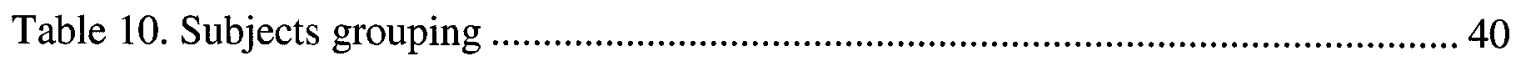

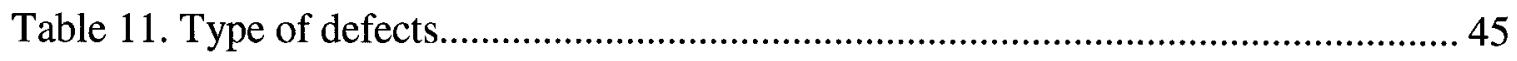

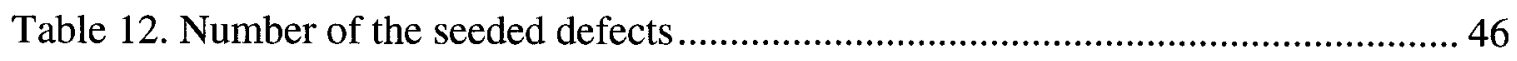

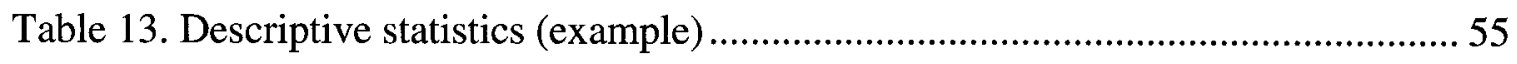

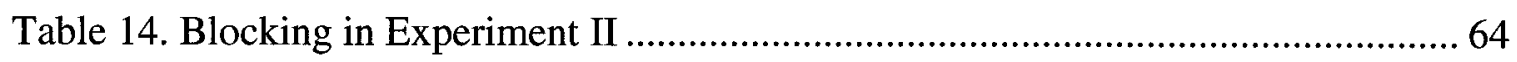

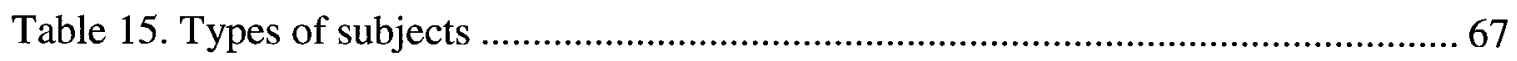

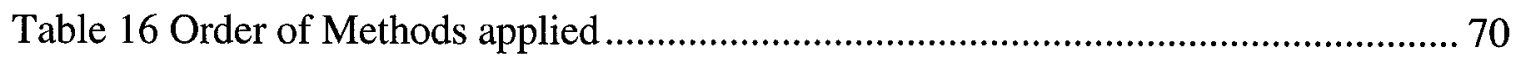

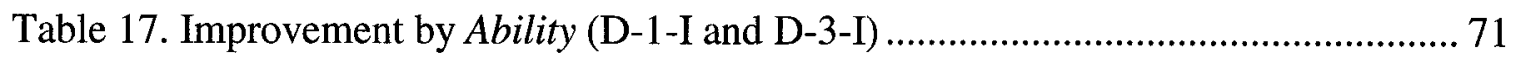

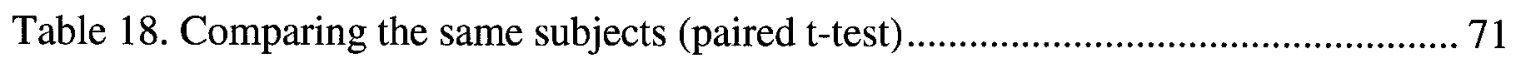

Table 19. Two-way ANOVA (Method \& Ability) - D-1-I …........................................ 73

Table 20. Descriptive statistics (Method \& Ability) - D-1-I......................................... 73

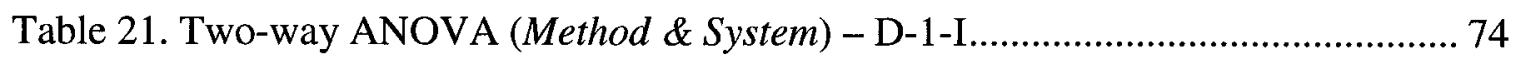


Table 22. Descriptive statistics (Method \& System) - D-1-I ...................................... 74

Table 23. Two-way ANOVA (Method \& Ability) - D-3-I ............................................ 76

Table 24. Descriptive statistics (Method \& Ability) - D-3-I........................................ 76

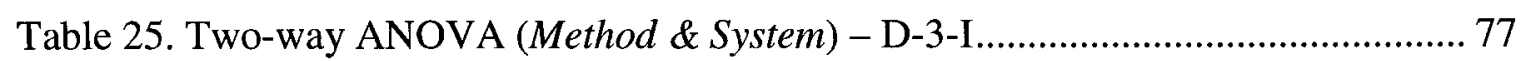

Table 26. Descriptive Statistics (Method \& System) - D-3-I........................................... 77

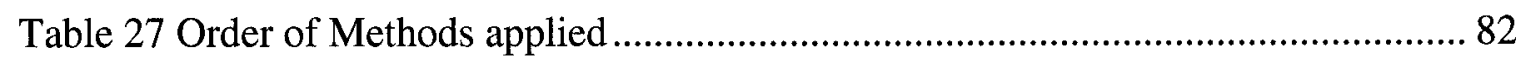

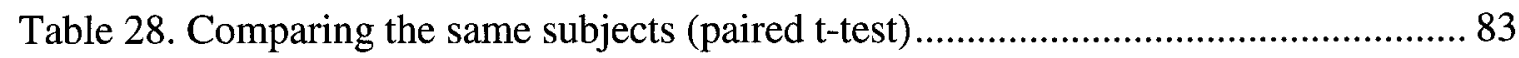

Table 29. Three-way ANOVA (Method, Ability \& System) - D-1-II............................ 85

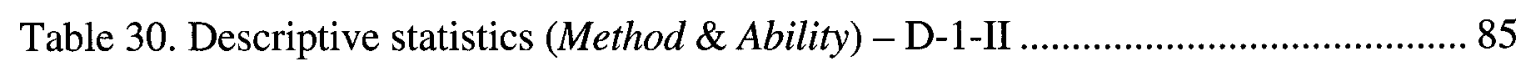

Table 31. Three-way ANOVA (Method, Ability \& System) - D-3-II.............................. 88

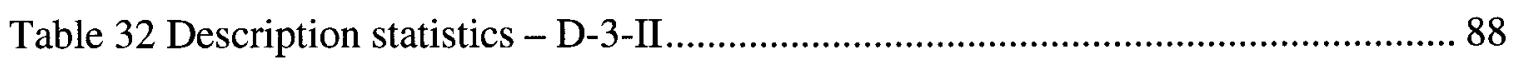

Table 33. All observation analysis of both experiment trials ......................................92

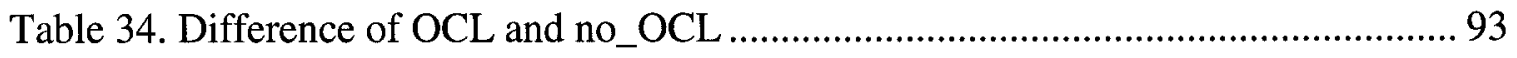

Table 35. Comparing Experiment I and Experiment II - Main effects .........................95

Table 36. Comparing Experiment I and Experiment II - Interaction effects ................. 95

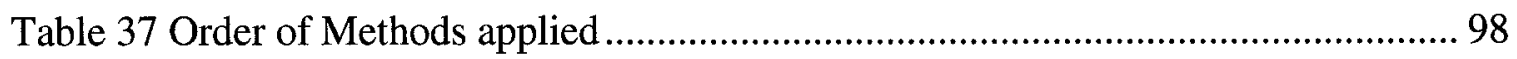

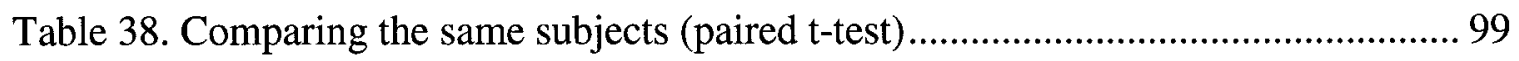

Table 39. Two-way ANOVA (Method and Ability) - C-2-I....................................... 100

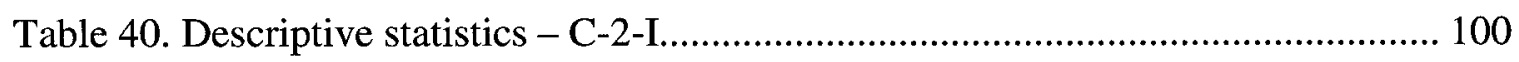

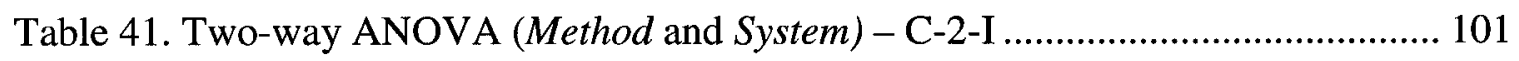

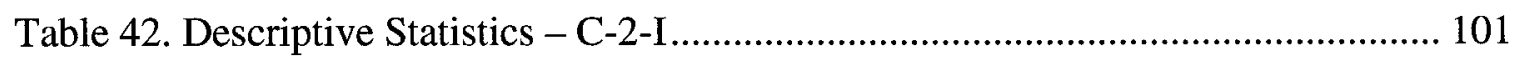

Table 43. Two-way ANOVA (Method \& Ability) - C-4-I ........................................ 103

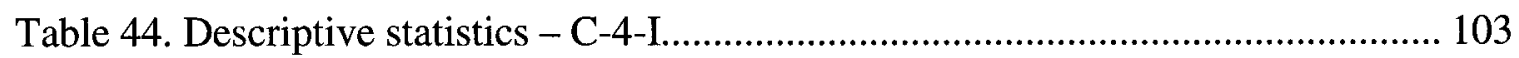

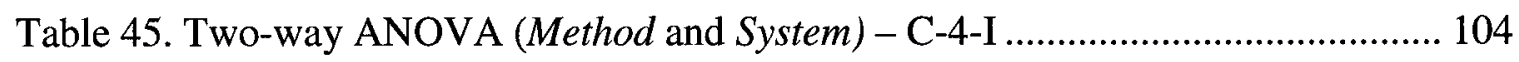


Table 46. Descriptive statistics - C-4-I................................................................ 104

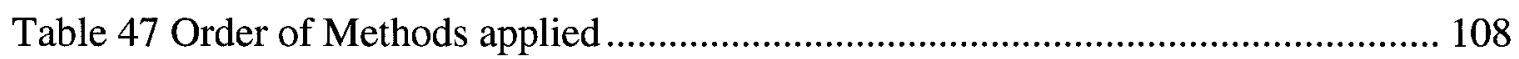

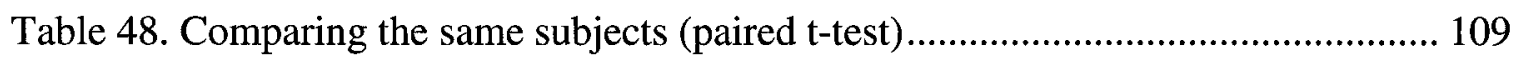

Table 49. Three-way ANOVA (Method, Ability \& System) - C-2-II.......................... 110

Table 50. Descriptive statistics - C-2-II ..................................................... 110

Table 51. Three-way ANOVA (Method, Ability \& System) - C-4-II ............................ 112

Table 52. Descriptive statistics - C-4-II .......................................................... 112

Table 53. All observations analysis of both experiment trials................................... 116

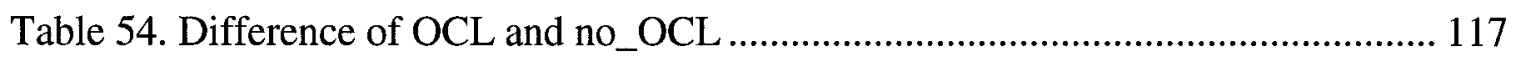

Table 55. Comparing Experiment I and Experiment II ........................................... 118

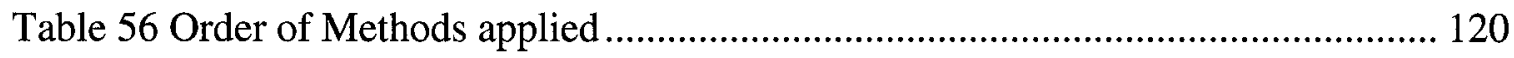

Table 57. Comparing the same subject (paired t-test) ........................................... 121

Table 58. Two-way ANOVA (Method and Ability) - M-2-I....................................... 122

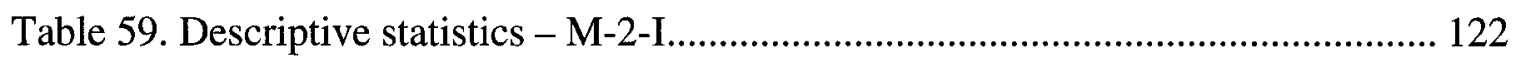

Table 60. Two-way ANOVA (Method \& System) - M-2-I ....................................... 123

Table 61. Descriptive Statistics- M-2-I............................................................ 123

Table 62. Two-way ANOVA (Method \& Ability) - M-4-I ........................................... 125

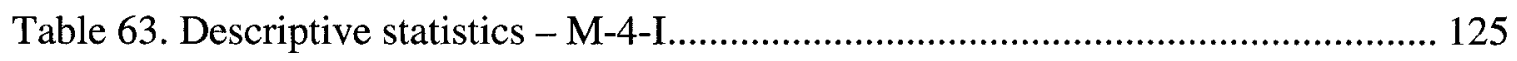

Table 64. Two-way ANOVA (Method and System) - M-4-I ................................ 126

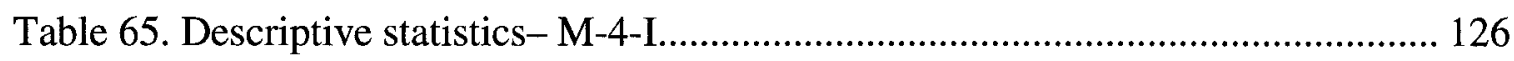

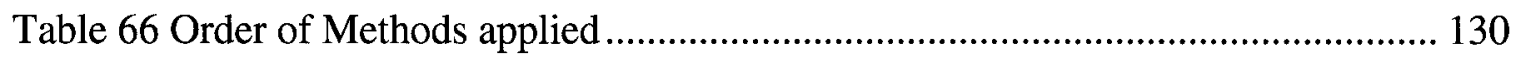

Table 67. Comparing the same subject (paired t-test) ......................................... 130

Table 68. Three-way ANOVA (Method, Ability \& System) - M-2-II ........................... 132

Table 69. Descriptive statistics- M-2-II ............................................................. 133 
Table 70. Three-way ANOVA (Method Ability \& System) - M-4-II ............................ 135

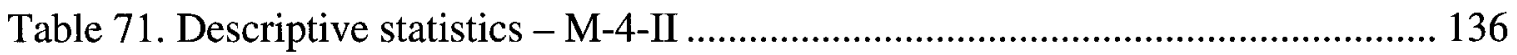

Table 72. All observations analysis of both experiment trials................................ 139

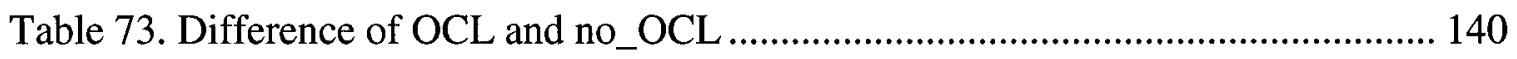

Table 74. Comparing Maintenance I and Maintenance II - Main effects .................... 141

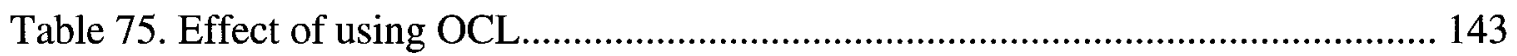

Table 76. Significant improvement by the subjects............................................... 146

Table 77 Summary of the significant effects .................................................. 147

Table 78. Defects seeded in the Cab Distribution System....................................... 161

Table 79. Defect seeded in the Video Store System ............................................... 162

Table 80. Defect Detection-1-I by Ability ...................................................... 180

Table 81. Defect Detection-3-I by Ability \& Method ................................................. 180

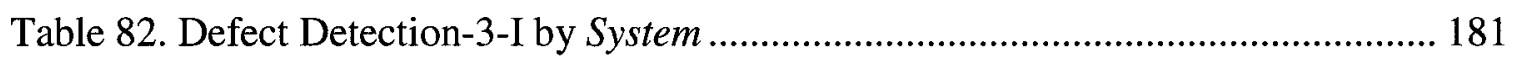

Table 83. Comparing Defect Detection-1-I and Defect Detection-3-I .......................... 181

Table 84. Comparing D-1-I and D-3-I - attitudes of OCL .................................... 181

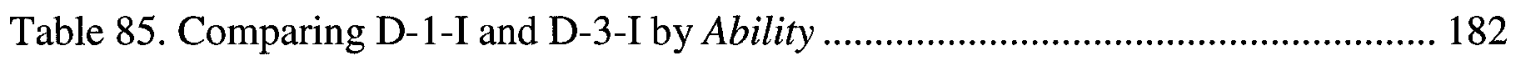

Table 86. Comparing D-1-I and D-3-I - attitudes of OCL by Ability ........................... 182

Table 87. Defect Detection-1-II by Ability ............................................................. 182

Table 88. Defect Detection-1-II by System ............................................................ 183

Table 89 Defect Detection-3-II by Method ............................................................. 183

Table 90 Defect Detection-3-II by Ability ............................................................... 183

Table 91 Defect Detection-3-II - attitudes of OCL by Ability ..................................... 184

Table 92 Comparing Defect Detection-1-II and Defect Detection-3-II........................ 184

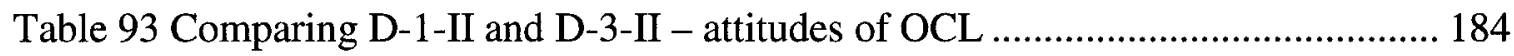


Table 94 Comparing D-1-II and D-3-II by Ability....................................................... 184

Table 95 Comparing D-1-II and D-3-II - attitudes of OCL by Ability............................ 185

Table 96. Comprehesnion-2-I and Maintenance-2-I by Method .................................... 185

Table 97. Comprehesnion-2-I and Maintenance-2-I by System........................................ 186

Table 98. Comprehesnion-4-I and Maintenance-4-I by Method ……............................. 186

Table 99. Comprehesnion-4-I and Maintenance-4-I by Ability ..................................... 187

Table 100. Comprehesnion-4-I and Maintenance-4-I by System...................................... 187

Table 101. Comparing 2-I and 4-I ......................................................................... 188

Table 102. Comparing 2-I and 4-I - attitudes of OCL ................................................. 188

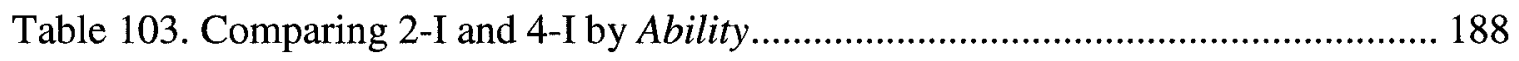

Table 104. Comparing 2-I and 4-I - attitudes of OCL by Ability.................................... 189

Table 105. Comprehesnion-2-II and Maintenance-2-II by Method .................................. 189

Table 106. Comprehesnion-2-II and Maintenance-2-II by System .................................. 189

Table 107. Comprehesnion-4-II and Maintenance-4-II by Method................................. 190

Table 108. Comprehesnion-4-II and Maintenance-4-II by Ability ................................. 190

Table 109. Comprehesnion-4-II and Maintenance-4-II by System .................................. 191

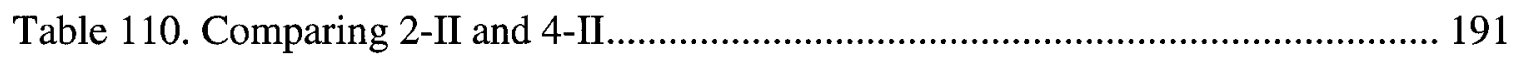

Table 111. Comparing 2-II and 4-II - attitudes of OCL................................................ 191

Table 112. Standard deviations and testing equal variance …….................................... 192 


\section{CHAPTER 1 INTRODUCTION}

The Object Constraint Language (OCL) $[13,18]$ was proposed as a way to bring additional precision to system analysis documents and/or design models described using the Unified Modeling Language (UML) notation. OCL is currently part of the UML standard $[1,14]$. However, a number of researchers recommend against using OCL $[11]^{1}$ or leave it out of their proposed methodologies [7]. Some recommend using OCL only during low-level design [4]. Very few industrial organizations, currently employing UML, make use of OCL.

Though it is clear that OCL brings precision to UML models and offers a number of potential benefits $[3,5,10]$, the question of whether the additional effort and formality associated with OCL bring any tangible benefits in practice, still remains to be answered. This is akin to the old, still on-going debate in software engineering regarding the degree of formality required in the early phases of high-quality software development.

This thesis reports on a controlled experiment performed in a university setting, in which whether the additional effort and formality of OCL brings tangible benefits in practice is assessed. The subjects are $4^{\text {th }}$ year computer and software engineering students who have been trained in UML-based software development over several courses. As further

\footnotetext{
1 "Unless there is a compelling practical reason to require people to learn and use the OCL, keep things simple and use natural language." [11]
} 
discussed below, the choice of performing a controlled experiment was made to control extraneous factors which could affect the results (e.g., students' ability) and to ensure that the subjects have sufficient knowledge of UML system analysis and OCL.

The need for a more scientific approach to Software Engineering was first emphasized in 1986 [26], as new techniques, methods, and languages were published and marketed without objective, quantified evaluation [23]. Scientific experimentation, which has been a common way to evaluate human-based activities in social and behavioral sciences for many years, can be applied to Software Engineering, as Software Engineering employs intensive human effort. Significant empirical research has been performed in the last twenty years $[20,23]$ to better understand issues and technologies related to large scale software development. In the present research context, only experimentation could provide a systematic, disciplined, quantifiable and controlled way of evaluating the impact of using OCL.

The experiment results show that the use of OCL, combined with UML, offers significant benefits, in terms of performing software engineering tasks effectively, such as defect detection, comprehension, and maintenance of UML analysis documents. However, the significant benefits are obtained only after a certain learning period.

This thesis is structured as follows: Chapter 2 provides background information regarding UML and OCL, an introduction to the concept and terminology of controlled experimentation and descriptions of the statistical tests used. An overview regarding a 
pilot experiment conducted to prepare for the actual experiment is provided as well. Chapter 3 provides details of the design of the experiment. Chapter 4 reports the results obtained and provides plausible interpretations. Conclusions in terms of significance of the results are drawn in Chapter 5, and future work is described in Chapter 6. 


\section{CHAPTER 2 BACKGROUND}

This chapter provides an overview of relevant concept related to the experiment reported herein. Section 2.1 introduces background information regarding UML \& OCL, and how the experimental idea originated. Section 2.2 introduces a pilot experiment, conducted to gain first hand experience with the material and the design of the experiment. Section 2.3 introduces general terminology and principles associated with experimental work. An overview of statistical techniques used in data analysis is provided in Section 2.4.

\subsection{UML and OCL}

OCL was first developed in 1995 by IBM during a business modeling project, and introduced together with UML in 1997 [18]. UML provides a standard modeling notation for object-oriented analysis and design activities. OCL, as part of UML, is a formal language specifying imposed constraints on modeled objects [18], so that functionalities of objects can be established clearly and unambiguously. Typical constraints include: invariants, operation contracts (pre-conditions and post-conditions) [24], formal path conditions in a sequence diagram, formal guard conditions in a statechart, etc. Invariants are conditions that must always be met by all instances of corresponding classes, types, or interfaces. Pre- and post-conditions must be true before and after an operation is 
executed, respectively. Other constraints, such as path conditions and guard conditions must be met whenever corresponding sequences and/or transitions occur.

Figure 1 shows an example of a typical UML operation specification in the context of a Video Store system. Operation cancelReservation of class ReservationControl cancels a video rental reservation. The operation takes one parameter reservationNo, which identifies the video rental reservation to be cancelled, and does not have any return value. However, such UML description does not show what type of reservation can be cancelled (e.g., can it be extended to canceling a reservation which is already cancelled?), nor does it show precise results of the operation (e.g., in terms of objects and relationships changed).

Figure 1. An example of UML operation specification

\section{ReservationControl::cancelReservation(reservationNo: String)}

Description: Executes the CancelReservation use case: a reservation will transit to state Cancelled

Parameter-List: reservationNo: a string uniquely representing the Reservation to cancel Return-List: None

OCL allows pre- and post-conditions to be described so that operations are defined more precisely. Pre- and post-conditions of the cancelReservation operation of the ReservationControl class can be described in OCL, as shown in Figure 2. Lines 1 to 4 describe the pre-conditions of the operation: there must exist a reservation that is in state \#Pending or \#Outstanding prior to the execution of the cancelReservation operation. Lines 5 to 34 describe the post-conditions of the operation: Lines 5 to 8 require that the reservation's state, after the execution of the 
operation, should be \#Cancelled. Lines 9 to 17 specify that if the cancelled reservation was the OldestPending reservation and there existed another pending reservation prior to the execution of the operation, the next pending reservation should become the oldestPending reservation after the execution of the operation. If no other pending reservation existed prior to the execution of the operation, the OldestPending reservation associated to the video title should be cleared after the execution of the operation. Lines 18 to 34 specify the conditions that must be met after the execution of the operation, if the cancelled reservation was an \#Outstanding reservation prior to the execution of the operation: if the associated video title had an oldestPending reservation, this reservation should be \#Outstanding after the execution of the operation, and its associated video copy should be set to the copy that used to be associated to the cancelled reservation. Meanwhile, if there existed a secondoldestPending reservation prior to the execution of the operation, the SecondoldestPending reservation should be the OldestPending reservation after the execution of the operation; otherwise the OldestPending reservation associated to the video title should be cleared once the operation is executed. If the cancelled reservation was not \#Outstanding prior to the execution of the operation, the associated video copy's state should be \#ForRent after the execution of the operation. 
Figure 2. Pre- / Post-condition of ReservationControl::cancelReservation in OCL

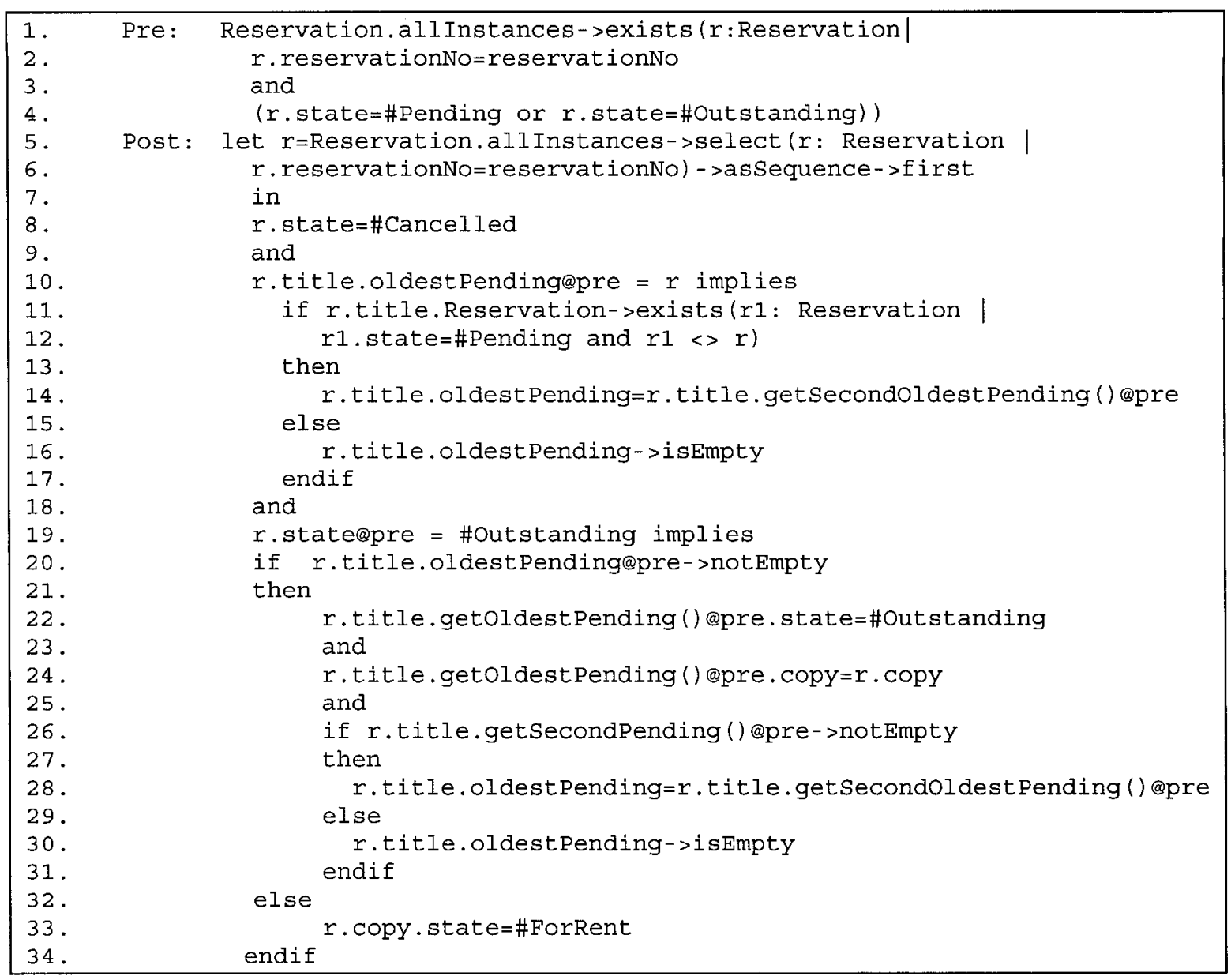

Similar information may be obtained from other parts of the UML document even if OCL is not used. For example, from the statecharts, one should be able to determine whether both \#Pending reservation and \#Outstanding reservation can be cancelled. Further, statecharts often specify what operations should be executed, after a state transition, by providing actions. Descriptions of operations for actions are often specified in a data dictionary. All the information, which is provided by OCL expressions, can be documented in a UML analysis document using natural language. However, this is not usually done. The precision and the level of details depend on the 
individual engineers analyzing and/or designing the system. Using OCL forces engineers to document UML system analysis more precisely by adding formality thereto , e.g., rather than being implicit, the entry criteria and consequence of an operation are unambiguously described by documenting pre- and post-conditions using OCL.

However, Figure 2 also implies that there would be an additional (possibly substantial) amount of effort to be put into system analysis, if OCL were used. Extra effort is required to learn, apply and insert OCL expressions in UML system analysis document.

Herein lies the on-going debate on whether or not the effort is worth the benefit. There are two aspects of this debate: (1) whether using OCL brings any tangible benefits in performing software engineering activities; and (2) whether the benefits are larger than the cost of using OCL. For the first aspect, it is possible that a UML system analysis document without OCL constraints is clear enough, so that there is no benefit in using OCL; it is also possible that the precision and formality of OCL allow engineers to spend less time in understanding the analysis and design of a system, maintaining the system, and detecting defects. For the second aspects, the cost of using OCL may or may not be justified, depending on whether it is less than the benefits derived from using OCL. The only way to settle the question, one way or the other, is through scientific experimentation. No known experiment has yet been conducted toward these ends. Thus, an experiment was performed to examine the usefulness of OCL to investigate the first aspect of the debate. This thesis reports on the design of the experiment and the results. More experiments should be carried out to investigate the second aspect of the debate. 


\subsection{The pilot experiment}

Prior to the formal experiment, a pilot experiment was conducted as part of a fourth year Computer and Software Engineering course to gain first-hand experience in using the material developed and in assessing the experiment design.

100 students worked in pairs on two UML analysis documents describing a Cab Distribution system (CD) and a Video Store system (VS). These UML system analysis documents include sets of consistent diagrams, such as Use Case diagrams, Class diagrams, Sequence diagrams, Statechart diagrams. Two analysis documents were used for each system: one version with OCL constraints (referred to as the UML+OCL version), and the other without (referred to as the UML-only version). For the UML+OCL version of the system analysis document, OCL was used to document the following aspects of the UML models: operation pre-conditions and post-conditions, class invariants, guard conditions in statecharts, and path conditions in sequence diagrams. For the UML-only version of the system analysis document, the abovementioned aspects were documented using natural language, or remained implicit.

All pairs of students participated in two laboratories. In each laboratory, the impact of using OCL was examined on three software engineering activities: detecting defects in the documents (Defect Detection task), understanding of the system analyses (Comprehension task), and maintaining the systems (Maintenance task). Defects were 
seeded in the system analysis documents for the Defect Detection task, and questions were prescribed for the Comprehension and the Maintenance tasks.

50 pairs of students were divided into two groups, so that they could work on a different system in a particular laboratory. As illustrated in Table 1, the experiment design ensures that all students have the opportunity to work on both systems, and to work without OCL first and then with OCL. After the two laboratories, a brief survey questionnaire was given to collect feedback from the students in terms of the usefulness of OCL on a scale of 1 to $5: 1$ being perceived useful and 5 being perceived useless.

Table 1. Design of the pilot experiment

\begin{tabular}{|c|c|c|}
\hline & Group 1 & Group 2 \\
\hline Lab 1 & Cab Distribution (without OCL) & Video Store (without OCL) \\
\hline Lab 2 & Video Store ( with OCL) & Cab Distribution (with OCL) \\
\hline
\end{tabular}

The performance of each pair of students was measured by evaluating their answers. Statistical tests (t-tests [6]) were used to analyze the performance data. (An overview of the statistical tests used in this thesis is provided in Section 2.4.) Results show that, for the Video Store system, the students performed better with the UML+OCL version of the analysis documents for the Comprehension and the Defect Detection tasks, while fared worse for the Maintenance task. For the Cab Distribution system, the students performed better with the UML+OCL version of the analysis documents for the Comprehension task. Using OCL did not make any difference between using UML+OCL and UML-only versions for the Maintenance and the Defect Detection tasks. 
The results of the pilot experiment are difficult to interpret due to problems with the experiment design and operation. These problems are discussed next and lead to change the setup of the experiment.

\section{Experiment design}

The experiment design, as shown in Table 1 , only enables limited statistical testing performed: testing the differences between using UML+OCL \& UML-only versions in each system (CD \& VS). It is difficult to determine if the improvement from first attempt at the Comprehension task to the second attempt seen is due to more UML knowledge gained over time, or to the use of OCL. Moreover, the results for the Cab Distribution system are different from for the Video Store system. It is difficult to determine whether the difference is due to differences in system characteristics or any other reason.

\section{The subjects}

The students worked in pairs. The disadvantage of this set-up is that the levels of students' ability can not be fully controlled. Some students may be more skilled in UML system analysis than others. When a more skilled student pairs with a less skilled student, whether such pair of students should be considered as being more skilled or less skilled cannot be determined with certainty. Thus, the possible interaction between students' skill levels and using OCL cannot be easily studied. Furthermore, some students changed partners between the laboratories despite close monitoring, and thus their performance data could not be used. 


\section{Time allocation}

Each laboratory was three hours long. The students used 90 minutes to detect defects, and 90 minutes to go through the Comprehension and Maintenance questionnaires. Many students did not finish the Maintenance questionnaire; some did not even finish the Comprehension questionnaire. The students did not seem to have enough time to digest the materials and finish the tasks. Thus the results may not represent the subjects' true performance, especially for the Maintenance task.

\section{Post-lab survey}

The post-lab survey was unfortunately too brief. The only conclusion from the data was that the students consider OCL to be useful overall. The survey does not provide information to help interpret the data analysis results.

\subsection{Experimental design terminology and principles}

Incorporating the lessons learned from the pilot experiment, an experiment was designed with two trials, as reported in this thesis. Section 2.3.1 introduces the experiment terminology [20] used in this thesis in the context of the pilot experiment mentioned above. Other terms will be introduced as needed. Important experiment design principles are introduced in Section 2.3.2. 


\subsubsection{Terminology}

The purpose of an experiment is to study the outcome of a process, when input variables are varied. The outcome of the process consists of dependent variables, while the inputs to the process are referred to as independent variables, or factors. The dependent variables' values may change according to the variation in the factors' values, the change being denoted as effect(s). The experiment therefore is conducted to study the effect of the factors on the dependent variables. In the pilot experiment, the studied factor is whether or not OCL is used, which is denoted as Method. The dependent variables are the participants' effectiveness at detecting defects, understanding the system, and maintaining the system, which is measured by the participants' performance.

Each factor has different values, which are referred to as treatments or levels. For example, using $O C L(O C L)$ and not using $O C L$ (no_OCL) are the two treatments of the factor Method. The subjects are experiment participants, who are selected from a studied population. The subjects are assigned to different groups, to which different treatments are assigned respectively. The subjects perform different tasks (e.g., detecting defects) on experiment objects, so that the effect of the factors can be observed (observations). In the pilot experiment, the studied population is the new B. Eng. graduates entering the software engineering profession; the subjects are the students who participate in the laboratories; and the objects are the UML system analysis documents. The assessment of 
the performance of each pair of students for every task is an observation. The number of observations is called the sample size.

\subsubsection{Experiment design principles}

In conducting an experiment, it is important to ensure that the intention of the experiment is fulfilled through an appropriate design of the experiment. When designing an experiment, the following principles must be considered: Randomization, Blocking, and Balancing [20, 23].

\subsubsection{Randomization}

Randomization is one of the most important experiment design principles. Undesirable effects of factors, other than the studied factors, may exist and may impact the experiment results. Randomization is used to ensure the characteristics of different treatment groups are similar, so that other factors' effects can be averaged out. Randomization should be applied on the allocation of: subjects, objects, order in which treatments are assigned, etc. For example, subjects should be assigned to treatment groups randomly. 


\subsubsection{Blocking}

Blocking is a technique used in scientific experiments to ensure that the effects of anticipated extraneous factors, that may be confounded with the effect of the studied factor, are controlled [20]. Although Randomization can also be used for this purpose, when the sample size is small, there still is a chance that the characteristics of different treatment groups may not be similar, which can be addressed by Blocking. Factors, whose effects are blocked, are called Blocking factors. Blocks are defined for each Blocking factor, according to the categorized characteristics. Subjects are then assigned to treatment groups randomly from each block. In the example of the pilot experiment, some students may be more skilled in UML system analysis than others. The students can be categorized as more-skilled and less-skilled. The more-skilled students can be randomly assigned to treatment groups first, then the less-skilled students. The number of more-skilled and less-skilled students should be similar in each treatment group.

\subsubsection{Balancing}

Balancing is another experiment design principle. It is not mandatory, but it simplifies and strengthens the statistical analysis of the data (further discussed in next section). Balancing is achieved via having equal or similar sample sizes for each treatment group. 


\subsection{Overview of the statistics tests used}

Hypothesis testing is used to determine whether the effect of a factor on dependent variables is statistically significant [21]. A null hypothesis and an alternative hypothesis are usually defined. The null hypothesis typically assumes that the effect does not exist, in other words, that there is no difference between the observations from subjects with different treatments. The alternative hypothesis assumes that the effect exists, e.g., that there is a difference between the observations from the subjects with different treatments (two-tailed hypothesis), or that the subjects with one treatment are better than the others (one-tailed hypothesis). The corresponding tests are called two-tailed tests, or one-tailed tests, respectively, depending on the type of the alternative hypothesis defined. If the null hypothesis can be rejected statistically, then the effect of the factor on the dependent variables is considered / referred to as statistically significant.

The following statistical tests are used to test the hypotheses in this experiment: the Student's t-test, the Mann-Whitney-Wilcoxon test, the paired t-test and ANOVA [21]. These are introduced in Sections 2.4.1 and 2.4.2.

\subsubsection{Student's t-test, Mann-Whitney-Wilcoxon test, and Paired t-test}

The Student's t-test, often simply called the $t$-test, is the most common statistical test for comparing the means of observations from two different treatment groups. Three statistical assumptions need to be satisfied when using this test: 
1. Independent groups of observations:

The groups of observations should be different random samples of the studied population.

2. Normal distribution:

The observations should have approximately bell curved distributions.

3. Equal variances:

The two groups of observations should have equal variances. In reality, the variances $\sigma$ of the two groups may not be exactly the same. The equality of the two variances can be tested by computing the ratio of the two variances. This ratio follows the F-distribution [9].

When it is not certain whether the above assumptions can be met, the Mann-WhitneyWilcoxon test, can be used [20]. This test will be referred to as the Wilcoxon test hereafter.

When assigning two different treatments to the same subjects, there are two observations for each subject, denoted as paired observations, which can be analyzed by testing differences between them using the paired t-test. Neither the t-test nor the Wilcoxon test can be used, as they require independent observations.

The $t$-test/Wilcoxon test and the paired t-test can be used to test a one-tailed hypothesis or a two-tailed hypothesis. In accordance with a typical $t$-test/Wilcoxon test or a paired $t$-test table, as shown in Table 2, the mean of the observations for each testing group of subjects and a p-value are reported, " $\mathrm{X}$ " is the mean value of observations of Group A, 
"Y" is the mean value of observations of Group B. (Note that the subjects of Group A and Group B must be the same in order to apply the paired t-test, but different in order to apply the $t$-test/Wilcoxon test.) A p-value is a measure of the probability that a difference between the groups happened by chance. For example, a $p$-value of 0.5 means there is a probability of $50 \%$ that the result occurred by chance. The lower the $p$-value, the more likely it is that the difference between groups is caused by the considered treatment. A pvalue of 0.05 is usually used as a significance threshold $(\alpha)$, in other words, $p$-values $\leq$ 0.05 are considered to be statistically significant.

Table 2. A typical t-test/Wilcoxon test table

\begin{tabular}{|c|c|c|}
\hline Mean (Group A) & Mean (Group B) & p-value \\
\hline X & Y & Z \\
\hline
\end{tabular}

\subsubsection{ANOVA}

When there is more than one factor to be studied, the researcher is often also interested in the interactions between the factors, which can only be studied via Analysis of Variance (ANOVA) [9].

ANOVA is used to assess whether observed differences among two or more sample means are statistically significant [9]. It cannot be used to test which group is better, however. If only one factor is studied, the corresponding ANOVA is a one-way ANOVA. If two or more factors are studied, say $\mathrm{n}$ factors, the corresponding ANOVA is an n-way ANOVA. 
ANOVA is a good supplement to a simple $t$-test, when more than one factor is studied. In the context of the pilot experiment, using OCL may have a different effect on moreskilled subjects than on less-skilled subjects, the interaction between using OCL and the subjects' skill level may be of interest. ANOVA allows an investigation of interaction effects between factors. Further, when ANOVA is used, the variance explained by the students' skill level is removed, and the analysis of the effect of using OCL becomes more powerful as a result, e.g., it is more likely to see a significant effect if there is one. Moreover, ANOVA is known to be very resilient to departures from the assumptions of independent groups, normally distributed observations, and equal variances [8], especially when the sample sizes are equal for each combination of factor treatments (e.g., more-skilled subjects who use OCL). If the differences in sample sizes are not too large, unequal sample sizes do not prevent the use of ANOVA, rather just more complex to interpret its results. The results may depend on the order in which factors are included in ANOVA. To overcome the disadvantage of having unequal sample sizes, the most important factor should be the last one to be included in ANOVA [27], so that the variance explained by the more important factors is calculated after the variation explained by the less important factors has been derived.

In applying the ANOVA analysis, the observations can be expressed in the following form: Observation $=$ sample mean + treatment effect + residual, where residual is the variation not explained by any factors or their interactions. Corresponding Sums of Squares (SS) of observations satisfy the same decomposition: SS(observation) = 
$S S($ sample mean $)+S S($ treatment effect $)+S S($ residual $)$. If there are no differences between the means of the observations for the different treatments, SS(treatment effect) and SS(residual) should be approximately equal. The ratio between the two, divided by their degrees of freedom (d.f.), respectively, follows the F-distribution. Therefore, an Ftest rejects the hypothesis of no difference in the means at significance threshold $\alpha$, if $\mathrm{F}=$ $\frac{S S(t r) / d . f .(t r)}{S S(r e s) / d . f .(\text { res })}>F_{\text {d.f.(tr), d.f.(res) }}(\alpha)$, where 'tr' denotes treatment effect, 'res' denotes residual [9].

A typical ANOVA table is shown in Table 3. Column Source lists all studied factors, their interactions, and the residual. From Table 3, the studied factors are Ability and Method. Their interaction is denoted as Ability * Method. Column $d . f$. lists the degrees of freedom of the corresponding sources. Column Sum of Square lists the sum of squares for each source. Values of column Mean Square are obtained by dividing each Sum of squares value by the corresponding d.f. values. The ratios between the $S S(t r)$ and the $S S($ res $)$ values divided by the corresponding d.f. values are listed in column $F$ Ratio. Column Prob $>F$ lists the $p$-values of the corresponding $F$ ratios. Effects of individual factors are referred to as Main effects. Effects of interactions between factors are referred to as Interaction effects. When a p-value is smaller than a pre-determined significance threshold $\alpha$, say 0.05 , the effect of corresponding factor or interaction is statistically significant at $\alpha=0.05$. In the example of Table 3, Ability has a significant main effect (p- 
value $\leq 0.05$ ). Method does not have a significant main effect ( $\mathrm{p}$-value $>0.05$ ). Ability and Method have a significant interaction effect between them ( $\mathrm{p}$-value $\leq 0.05$ ).

Table 3. Example ANOVA table

\begin{tabular}{|l|l|l|l|l|l|l|}
\hline Source & DF & Sum of Squares & Mean Square & F Ratio & Prob $>$ F & $E^{2}$ \\
\hline Ability & 1 & 0.04858585 & 0.04858585 & 6.2976 & $\mathbf{0 . 0 2 1}$ & 0.20 \\
\hline Method & 1 & 0.00001799 & 0.00001799 & 0.0023 & 0.962 & 0.0001 \\
\hline Ability*Method & 1 & 0.04607644 & 0.04607644 & 5.9723 & $\mathbf{0 . 0 2 4}$ & 0.19 \\
\hline Residual & 20 & 0.15430028 & 0.007715 & & & 0.64 \\
\hline Total & 23 & 0.2418253 & & & & \\
\hline
\end{tabular}

Another standard statistical measurement is the correlation ratio $\left(\mathrm{E}^{2}\right)$. It measures the strength of the relationship between each of the factors and the dependent variables, representing the relative improvement in the predictability of the value of the dependent variables when it is known what group an observation belongs to. In other words, the correlation ratio tells how much of the variation in the dependent variables is explained by a particular factor [8]. The correlation ratio of a factor can be derived from ANOVA table by dividing the Sum of Square value of the corresponding factor by the total Sum of Square value. 


\section{CHAPTER 3 THE EXPERIMENT}

The structure of this chapter follows the template provided in Experimentation in Software Engineering - An Introduction [20], a well-known textbook on software engineering experimentation. Two experiment trials have been conducted. The first trial is referred to as Experiment I, the second as Experiment II. Sections 3.1 to 3.8 describe Experiment I, while differences between Experiment $I$ and Experiment $I I$ are discussed in Section 3.9. Section 3.1 gives a definition of the experiment. Section 3.2 introduces the context of the experiment. Section 3.3 concerns the tested hypotheses. Section 3.4 shows how subjects are selected. Section 3.5 describes the design of the experiment. Section 3.6 introduces the materials used in the experiment. Section 3.7 describes the data analysis procedure. Section 3.8 discusses the validity of the experiment design.

\subsection{Experiment Definition}

The purpose of the experiment is to evaluate the impact of using OCL in combination with UML analysis (class diagrams, statecharts, sequence diagrams, etc.), on the effectiveness of software engineering activities.

The studied factor concerns the use of OCL, denoted as Method. There are two treatments of Method: $O C L$ and no_OCL, corresponding to OCL expressions being present in the UML analysis documents or not. Three typical software engineering 
activities are performed ${ }^{2}$ : understanding the system analysis document, modifying the system analysis document $[4,12]$, and detecting defects in the system analysis document.

\section{Understanding a system analysis document}

Once the system analysis phase has been completed, software development moves on to design and implementation phases. Unless the project is very small, it is unlikely that the engineers who performed the system analysis will be the only ones doing design and coding. Other engineers have to understand the system analysis, and clarify ambiguities before actual system design can begin. The discussion presented in section 2.1 shows that clarity is not always achieved by employing the UML notation alone. If OCL expressions help engineers to understand the system in a shorter time, then using OCL may save the clarification effort, ensuring that the system analysis is understood from the very beginning; which is always desirable from a project management's point of view. Furthermore, analysis errors, and/or misunderstanding of the analysis, are very expensive if these are not caught at the early stage of development.

\footnotetext{
${ }^{2}$ There are other software engineering activities, such as quality assurance, which are not studied in this experiment.
} 


\section{Modifying a system analysis document}

During software development, it is common for system requirements to change. The system analysis needs to be modified accordingly to facilitate implementation changes. A small change in a class may lead to many other related changes, such as changing states of other classes, pre- / post-conditions of operations, etc. Having a clear picture of the impact of a change reduces the chance of introducing faults. Since UML system analysis alone does not always clarify ambiguities, the impact of a change may not be identified easily. If the presence of OCL expressions helps to point out the impact of a change easily, correctly, and quickly, then the effort of documenting OCL expressions would be justified.

\section{Detecting defects}

Defects may exist in a UML system analysis document, even if the analysis document is produced by experienced engineers. It could be due to misunderstanding of the system requirements, miscommunication among team members, or human mistakes. Usually, the UML system analysis document is inspected by other engineers for correctness and completeness. From project management's perspective, it costs less to detect defects during early analysis stages than during subsequent development stages. If the existence of OCL expressions in a UML system analysis document helps inspecting engineers to detect more defects within time constraints, the effort of inserting OCL expressions in the UML system analysis document would be justified. 
Three dependent variables are defined to measure the effectiveness of the three abovementioned software engineering activities. The effect of the factor Method (using OCL or not) on these three dependent variables is the objective of this experiment:

- Comprehension $(C)$ : The Comprehension dependent variable captures the level of understanding of the system analysis;

- Maintainability $(M)$ : The Maintenance dependent variable captures the extent to which software engineers can identify the impacts of a change in a correct and complete manner; and

- Defect Detection (D): The Defection Detection dependent variable captures the extent to which software engineers can detect defects in a system analysis document.

\subsection{Context selection}

The context of the experiment is a fourth year Computer and Software Engineering course at Carleton University, Ottawa, Canada. The students have been trained in UMLbased object-oriented software development for at least three previous courses, with an increasing focus on software modeling. The focus of this course is to apply what has been learned in the context of a well-defined software development process, including system analysis and design phases.

Experimental tasks are performed on system analysis documents. System analysis documents are chosen because these represent the first UML models to be produced in 
accordance with most software development methodologies. System analysis documents are different from subsequent design documents in that architectural information is not provided therein while focusing mostly on the system logical structure and behavior. However, system analysis documents describe an emerging class structure which represents the starting point of the design phase. (These system analysis documents are typically incomplete as only application domain classes are documented.) System analysis is considered to be a critical stage of software development as all subsequent design and implementation phases depend on it.

The two system analysis documents relate to two real world applications, but are not further developed for any real use beyond the experiment, because of the experiment time constraints. The two analysis documents do not contain a complete analysis model: not all Use Cases have sequence diagrams present in the analysis documents. However, they address the following real life problems: the Cab Distribution (CD) system distributes cabs to customers, and the Video Store (VS) system handles video rentals and sales. Both systems were selected because they have an adequate level of complexity and represent familiar application domains for the subjects. All relevant aspects of a UML system analysis are touched upon for the purpose of the experiment including: Use Case Diagrams with all use cases and Use Case Descriptions; Sequence Diagrams of a subset of the use cases; a subset of Class Diagrams, including all relevant operations and their data dictionary used in the Sequence Diagrams; and a subset of Statecharts and their descriptions. 


\subsection{Hypothesis formulation}

The following null hypothesis $\left(\mathrm{H}_{0}\right)$ is formulated for testing the effect of Method on each dependent variable: There is no difference in Comprehension $(C)$, Maintenance $(M)$, and Defect Detection (D) effectiveness between the subjects working on UML system analysis documents using or not using OCL. The alternative hypothesis $\left(\mathrm{H}_{\mathrm{a}}\right)$ is that using OCL improves effectiveness for all three dependent variables. The formal definition is provided in Table 4. The rows present the three studied dependent variables. The columns present the null hypothesis and the alternative hypothesis for the corresponding dependent variable.

Table 4. Experiment hypotheses

\begin{tabular}{|l|l|l|}
\hline Dependent variable & Null hypothesis & Alternative hypothesis \\
\hline Comprehension & $H_{0}: C(O C L)=C($ no_OCL $)$ & $H_{a}: C(O C L)>C\left(n o \_O C L\right)$ \\
\hline Maintenance & $H_{0}: M(O C L)=M($ no_OCL $)$ & $H_{a}: M(O C L)>M\left(n o \_O C L\right)$ \\
\hline Defect Detection & $H_{0}: D(O C L)=D\left(n o \_O C L\right)$ & $H_{a}: D(O C L)>D\left(n o \_O C L\right)$ \\
\hline
\end{tabular}

\subsection{Selection of subjects}

The subjects are selected as the students registered in this last, most advanced software engineering course, as it is desired that the subjects possess a reasonable level of technical maturity and knowledge for UML-based, object-oriented software development. The students are familiar with concepts such as pre- and post-conditions, state invariants, and class invariants. There is no need to screen students further as variations in ability are also present in industrial settings. As most of these students will actually become certified 
engineers within a year, they represent the population of new graduates entering the software engineering profession.

The experiment is part of a series of compulsory laboratory exercises. The students are told that they are not graded on performance but that they are expected to perform their tasks individually in a professional manner to obtain the points assigned to the laboratory. All the students are monitored by the experimenters as the tasks are performed. The students are aware that the attempt is to evaluate the impact of using OCL as well as to improve their practical training in UML and OCL, but they are not aware of the exact hypotheses tested or what particular dependent variables are of interest. The subjects are not allowed to communicate with each other during the laboratories, and are required to hand in all experimental materials after each laboratory.

\subsection{The design of the experiment}

The experiment is carefully designed, incorporating lessons learned from the pilot experiment. Section 3.5.1 defines the tasks performed and time allocated. Section 3.5.2 discusses other undesirable factors, whose effects may confound with the effect of using OCL. Section 3.5.3 describes the experiment design and how the extraneous factors are controlled. 


\subsubsection{Experimental tasks and time allocation}

Three tasks are defined so that the dependent variables can be studied respectively: Defect Detection, Comprehension, and Maintenance tasks. Since it was observed that the subjects did not have enough time to complete the tasks during the pilot experiment, more time is allocated to each task in this experiment. The tasks and their time allocations are discussed below.

The Defect Detection Task is formulated to study the dependent variable Defect Detection. Thirty defects are seeded in each system analysis document, and the subjects are asked to find all the seeded defects during a time period of 150 minutes without knowing how many defects there are. The subjects' performance for this task is measured as the percentage of seeded defects detected.

The Comprehension Task is formulated to study the dependent variable Comprehension. Twenty comprehension multiple choice questions are formulated for each system. The subjects are asked to select one correct choice for each question during a time period of 90 minutes. The subjects' performance for this task is measured as the percentage of correctly answered comprehension questions.

The Maintenance Task is formulated to study the dependent variable Maintenance. A list of system changes is prescribed for each system. 71 model elements are affected in the Cab Distribution system, while 60 model elements are affected in the Video Store system. The subjects are asked to identify all the model elements affected by the system 
changes during a time period of 60 minutes, without knowing how many of the modeled elements should be affected. The subjects' performance for this task is measured as the percentage of affected model elements correctly identified, according to the prescribed system changes.

The Comprehension task is believed to be simpler than the Defect Detection and the Maintenance tasks, and more fundamental, as the latter two tasks require understanding of the systems. As the subjects are required to perform the three tasks on the same system document, Defect Detection has to be performed first, so that the subjects will use the document version with seeded defects before using the correct version.

Due to time limitations imposed on each laboratory, the Defect Detection task is completed in one laboratory, while the Comprehension and Maintenance tasks are completed in another, with the Comprehension task being performed before the Maintenance task. More time is allocated to the Comprehension task (90 minutes) than to the Maintenance task (60 minutes), as it is assumed that a thorough understanding of each system would help the subjects in performing the subsequent Maintenance task.

\subsubsection{Other factors to be controlled}

Method is the only factor of interest in this experiment. However, other factors may also impact the subjects' performance in an undesirable way, as these extraneous factors may 
confound Method's effect. Extraneous factors need to be controlled so that only the effect due to the factor Method, if there is any, is discernable.

\subsubsection{The subjects' academic background and OCL experience}

Some subjects may come from a program, such as Electrical Engineering, where the software development training received may not have been as intensive compared to that received by subjects coming from a Software/Computer Engineering program. Further, some subjects may have learned OCL prior to this course, while others may have not. Differences in subjects' academic background and the level of prior OCL knowledge may have an impact on the subjects' performance, e.g., Electrical Engineering students who were never exposed to OCL before, are likely to perform worse than others. These factors are denoted as Academic background and OCL experience, respectively.

\subsubsection{The ability of the subjects}

The subjects may have different levels of understanding of UML, OCL, and objectoriented system analysis. Their capabilities of performing the tasks are different. In addition, some subjects may be more dedicated than others. Subjects with better understanding of UML, OCL, and object oriented analysis, and more dedication are likely to perform better than others. This factor is denoted as Ability. 


\subsubsection{The order in which the subjects use and not use OCL when performing the tasks}

As indicated in relation to the pilot experiment, the order in which the subjects are exposed to OCL in a system analysis may result in an artificial interaction effect between the system used and the method used. For example, when subjects perform a task for the first time without using OCL and then perform the same task again using OCL, if there is any improvement observed in connection with the second attempt, compared to the first attempt, the improvement can be due to using OCL, or simply an increase of the subjects' knowledge with respect to general system analysis. This can be studied by considering the order in which the subjects apply the method [23]: using OCL first then not using OCL, or the reverse. This factor is denoted as Order of Method hereafter.

\subsubsection{The analyzed systems}

If there is only one analyzed system, the subjects' performance may depend on the depth of their knowledge in the field of the application. For example, if a subject knows more about Cab Distribution than other subjects, the subject is likely to perform better than the others, regardless whether OCL is used or not. Further, system complexity may also have confounding effect with the Method factor. For example, the effect of the use of OCL may be more discernable in a more complex system, or the other way around. This factor is denoted as System. 


\subsubsection{Choice of experiment design}

This section presents the design of the experiment in the context of Experiment I. As only the Method factor is of interest, a one-factor randomized design [20] can be used, in which the subjects are assigned to the treatment groups randomly. However, since our sample size is not large, there is a chance that the characteristics of the two treatment groups may be different, e.g. one group may have more high ability subjects than the other, resulting in confounding the effect of using OCL. Thus, Blocking is also used to assure that the anticipated extraneous factors are controlled, e.g. each treatment group has similar number of high ability subjects and low ability subjects. Blocks are then defined, so that the subjects can be assigned to treatment groups randomly from each block. Therefore, the design of this experiment is a one-factor block design [20]. The formation of the one-factor randomized design is presented in Section 3.5.3.1, and the Blocks used in this experiment are described in Section 3.5.3.2. Section 3.5.3.3 discusses another effect that is associated with the experiment design.

\subsubsection{One-factor randomized design}

A one-factor randomized experiment design is typically used, when there is only one factor of interest, in association with which the subjects are randomly assigned to different groups to perform tasks related to different treatments [20]. The studied factor Method has two treatments: $O C L$ and no_OCL. Thus two groups are a priori needed. It is also desirable to have the subjects performing the same tasks more than once (repeated 
measurements) using and not using OCL. The advantage is to maximize the number of observations, and to enable using the paired t-test to remove individual variations [21]. Table 5 shows a one-factor randomized experiment design with repeated measurements. The subjects are divided into two groups: Group A and Group B. Treatment OCL is assigned to Group A, and treatment no_OCL to Group B. Both groups perform the Defect Detection task in Lab 1, and the Comprehension and Maintenance tasks in Lab 2 (first attempt). The subjects repeat the same tasks with different treatments in Lab 3 and Lab 4 (second attempt). The effect of Order of Method is also controlled through this setup, as the subjects are assigned to different treatments in different orders.

Table 5 Preliminary one-factor experiment design with repeated measurements

\begin{tabular}{|c|c|c|c|}
\hline & Task & Group A & Group B \\
\hline Lab 1 & Defect Detection & OCL & no_OCL \\
\hline Lab 2 & Comprehension, Maintenance & OCL & no_OCL \\
\hline Lab 3 & Defect Detection & no_OCL & OCL \\
\hline Lab 4 & Comprehension, Maintenance & no_OCL & OCL \\
\hline
\end{tabular}

As there is no value to repeat the tasks on a same system, two different systems are used: the Cab Distribution system (CD) and the Video Store system (VS). One possible experimental design is to have all the subjects working on the CD system in Lab 1 and Lab 2, while on the VS system in Lab 3 and Lab 4. This setting has a disadvantage: the paired t-test cannot be performed, as the subjects' response to different systems may confound the effects of using OCL.

Thus, an experimental design shown in Table 6 is set up: Group A is further divided into Group 1 and Group 3, while Group B into Group 2 and Group 4. Group 1 and Group 2 
use the Cab Distribution system analysis document in Lab 1 and Lab 2, and the Video Store system in Lab 3 and Lab 4. Group 3 and Group 4 use the system analysis documents in reverse. The treatment $O C L$ is assigned to Group 1 and Group 3 in Lab 1 and Lab 2, and treatment no_OCL in Lab 3 and Lab 4. The treatments are assigned to Group 2 and Group 4 in reverse.

Table 6. Intermediate experiment design

\begin{tabular}{|c|c|c|c|c|c|}
\hline & Task & Group 1 & Group 2 & Group 3 & Group 4 \\
\hline Lab 1 & Defect Detection & $\mathrm{CD}(\mathrm{OCL})$ & $\mathrm{CD}($ no_OCL) & VS(OCL) & VS(no_OCL) \\
\hline Lab 2 & $\begin{array}{c}\text { Comprehension, } \\
\text { Maintenance }\end{array}$ & $\mathrm{CD}(\mathrm{OCL})$ & $\mathrm{CD}$ (no_OCL) & VS(OCL) & VS(no_OCL) \\
\hline Lab 3 & Defect Detection & VS(no_OCL) & VS(OCL) & CD(no_OCL) & CD(OCL) \\
\hline Lab 4 & $\begin{array}{c}\text { Comprehension, } \\
\text { Maintenance }\end{array}$ & VS(no_OCL) & VS(OCL) & CD(no_OCL) & CD(OCL) \\
\hline
\end{tabular}

It is also observed that the Video Store system is somewhat more complex than the Cab Distribution system, having, in comparison, substantially more associations, attributes and OCL clauses, as shown in Table 7. 
Table 7. The complexity of the analysis Documents

\begin{tabular}{|l|c|c|}
\hline & CD & VS \\
\hline \# of actors & 7 & 8 \\
\hline \# of use cases & 10 & 12 \\
\hline \# of classes & 20 & 18 \\
\hline \# of classes with inheritance & 1 & 0 \\
\hline \# of inheritance depth & 1 & 0 \\
\hline \# of associations & $\mathbf{5}$ & $\mathbf{8}$ \\
\hline \# of inheritance relationship & 2 & 0 \\
\hline \# of operations & 55 & 66 \\
\hline \# of attributes & $\mathbf{2 3}$ & $\mathbf{4 0}$ \\
\hline \# of states & 9 & 10 \\
\hline \# of state transitions & 16 & 15 \\
\hline \# messages & 48 & 39 \\
\hline \# of OCL clauses & $\mathbf{1 7 4}$ & $\mathbf{2 9 1}$ \\
\hline \# of page (with OCL) & 41 & 47 \\
\hline \# of page (without OCL) & 37 & 39 \\
\hline
\end{tabular}

OCL constraints are Boolean expressions [18], composed of OCL clauses connected by logical operators, such as and, or, imply. Each OCL clause may contain OCL sub-clauses connected by logical operators. \# of OCL clauses is the total number of OCL clauses / OCL sub-clauses without any logical operators documented in the system analysis documents.

The experiment design presented in Table 6 ensures that System does not have any confounding effect with Method. However, the design has a problem. Group 2 and Group 4 would use OCL two laboratories later than Group 1 and Group 3. Groups 2 and 4 would have more time to gain additional experience in UML and OCL due to activities prescribed in the course curriculum. The experiment design therefore may favor Group 2 and Group 4. On the other hand, the OCL knowledge that Group 1 and Group 3 gained in 
the first two laboratories (first attempt) may help them perform better in the later laboratories (second attempt), e.g. using OCL may help the subjects to gain more knowledge of the system analysis. In other words, the order in which Method treatments are applied within each attempt may also undesirably impact the results.

The experiment design is then modified, as shown in Table 8. Each subject works individually on each of the two systems, using UML+OCL and UML-only respectively. Each subject performs all the Defect detection, Comprehension, and Maintenance tasks on both systems in different laboratories, which are held once a week. The laboratories are spread over a four week period.

\section{Table 8. The experiment design}

\begin{tabular}{|l|l|l|l|l|l|l|}
\hline & Task & Group 1 & Group 2 & Group 3 & Group 4 & Week \\
\hline Lab 1 & $\begin{array}{l}\text { Defect } \\
\text { Detection }\end{array}$ & $\begin{array}{l}\text { CD } \\
\text { (OCL) }\end{array}$ & $\begin{array}{l}\text { CD } \\
\text { (no_OCL) }\end{array}$ & $\begin{array}{l}\text { VS } \\
\text { (OCL) }\end{array}$ & $\begin{array}{l}\text { VS } \\
\text { (no_OCL) }\end{array}$ & 1 \\
\hline Lab 2 & $\begin{array}{l}\text { Comprehension, } \\
\text { Maintenance }\end{array}$ & $\begin{array}{l}\text { CD } \\
\text { (no_OCL) }\end{array}$ & $\begin{array}{l}\text { CD } \\
\text { (OCL) }\end{array}$ & $\begin{array}{l}\text { VS } \\
\text { (no_OCL) }\end{array}$ & $\begin{array}{l}\text { VS } \\
\text { (OCL) }\end{array}$ & 2 \\
\hline Lab 3 & $\begin{array}{l}\text { Defect } \\
\text { Detection }\end{array}$ & $\begin{array}{l}\text { VS } \\
\text { (no_OCL) }\end{array}$ & $\begin{array}{l}\text { VS } \\
\text { (OCL) }\end{array}$ & $\begin{array}{l}\text { CD } \\
\text { (no_OCL) }\end{array}$ & $\begin{array}{l}\text { CD } \\
\text { (OCL) }\end{array}$ & 3 \\
\hline Lab 4 & $\begin{array}{l}\text { Comprehension, } \\
\text { Maintenance }\end{array}$ & $\begin{array}{l}\text { VS } \\
\text { (OCL) }\end{array}$ & $\begin{array}{l}\text { VS } \\
\text { (no_OCL) }\end{array}$ & $\begin{array}{l}\text { CD } \\
\text { (OCL) }\end{array}$ & $\begin{array}{l}\text { CD } \\
\text { (no_OCL) }\end{array}$ & 4 \\
\hline
\end{tabular}

Thus, the experiment design eliminates the undesirable effects of the Order of method and System factors on the dependent variables.

To satisfy the Randomization design principle, the method applied, the systems used, and in which order the method is applied, are assigned to the four groups randomly. The subjects are then randomly assigned to the four groups. 


\subsubsection{One factor block design}

Three other anticipated factors have to be controlled also: Academic background, $O C L$ experience, and Ability. They cannot be controlled by the experiment design, as they are characteristics of individual subject, thus cannot be assigned. They could be controlled by Randomization, if the sample size is large. In this experiment, Blocking is applied to ensure they are adequately controlled, so that the characteristics of the treatment groups are similar to the extent possible. Table 9 shows the blocks defined.

Table 9. Blocks defined for Experiment I

\begin{tabular}{|l|l|c|}
\hline Blocking factors & Blocks & Size of the blocks \\
\hline Academic background & Software engineering and Computer systems & 37 \\
\cline { 2 - 3 } & Electrical engineering & 1 \\
\hline \multirow{2}{*}{ OCL experience } & Learned OCL in the prerequisite course & 19 \\
\cline { 2 - 3 } & Learn OCL in the concurrent course & 19 \\
\hline $\begin{array}{l}\text { Ability } \\
\text { (Grade of prerequisite } \\
\text { course) }\end{array}$ & B- and above & 20 \\
\cline { 2 - 3 } & Below B- & 18 \\
\hline
\end{tabular}

The subjects are from Software Engineering, Computer Systems, and Electrical Engineering programs. The software engineering training in the Electrical Engineering program may not be as intensive as that provided in the Software Engineering program. Electrical Engineering students are likely to have less experience with, and less motivation to master, UML and OCL, since their motivation may be just to pass a mandatory course, rather than to apply the acquired knowledge in the future as software engineers. On the other hand, since software engineering is a critical part of the Computer Systems program, the subjects from the Computer Systems program are trained in software engineering just as much as those from the Software Engineering program. 
Thus, two blocks are formed for the Academic background factor: Software Engineering and Computer Systems, and Electrical Engineering. This blocking addresses the problem of having experiment groups with different levels of software engineering strength. In Experiment I, however, there is only one subject coming from the Electrical Engineering program, thus blocking of the Academic background factor is of little use, but should not be forgotten in case where larger proportions of subjects come from different programs.

Some subjects have learned OCL in prerequisite course, others have to learn OCL as part of the concurrent course. There is only one lecture allocated for introduction of OCL in the concurrent course, wherein training is provided without any practice. Subjects who learned OCL in the prerequisite course are likely to have a better understanding of OCL than the others, as they have had more time to mature their OCL knowledge. Thus two blocks are formed for the OCL experience factor: Learned $O C L$ in the prerequisite course, and Learn OCL in the concurrent course. This blocking assures the OCL experience distribution is similar for all groups.

As discussed in section 3.5.2.2, the Ability factor represents the subjects' UML system analysis knowledge and their dedication to master UML and OCL. Although not perfect, grades are a relatively good representation of each subjects' ability. Students with better grades gained in a previous (prerequisite) course teaching UML notation are expected to be better at using UML than others. Two blocks are formed for the Ability factor: grade B- and above (High Ability), and below grade B- (Low Ability). The reason why a grade of B- was chosen is that it was found to be the median grade of subjects. 
All subjects are blocked according to the above blocks. Only two blocks are formed for each blocking factor, as the sample size is not very large, and more blocks may not be appropriate. These blockings eliminate possible confounding effects between factors Academic background, OCL experience, Ability and the studied factor Method.

Thus, 38 students are assigned to four groups randomly from each block. The sample sizes for the treatment groups are approximately equal to satisfy the experiment design principle Balancing. Table 10 shows the distribution of $O C L$ experience and Ability within each group.

Table 10. Subjects grouping

\begin{tabular}{|c|c|c|c|c|}
\hline & \multicolumn{2}{|c|}{ OCL Experience } & \multicolumn{2}{c|}{ Ability } \\
\hline & $\begin{array}{c}\text { Learned prior } \\
\text { to the course }\end{array}$ & $\begin{array}{c}\text { Learned in } \\
\text { the course }\end{array}$ & High & Low \\
\hline Group 1 & 5 & 5 & 5 & 5 \\
\hline Group 2 & 4 & 5 & 5 & 4 \\
\hline Group 3 & 5 & 5 & 5 & 5 \\
\hline Group 4 & 5 & 4 & 5 & 4 \\
\hline Total & 19 & 19 & 20 & 18 \\
\hline
\end{tabular}

\subsubsection{Learning effects}

The experiment design causes another effect that may also confound the effect of using OCL: a learning effect, which is resulted from a repeated task performance: the subjects perform the three tasks twice, and use UML analysis documents of two different systems. The subjects are likely to learn from the first attempt at the tasks, resulting in a performance improvement in the second attempt. The learning effect may confound the 
effect of using OCL. For instance, for those subjects who do not use OCL during the first attempt at a task, and use OCL during the second attempt, if a performance improvement is observed between the first attempt and second attempt, the improvement may be due to the effect of using OCL. However, it may also be due to the learning effect, or to the effects of both using OCL and learning. Thus, the learning effect must be taken into account when interpreting the data analysis results to avoid false conclusions.

\subsection{Instrumentation}

The materials used in an experiment are referred to as the instruments. An instrument preparation strategy is introduced in Section 3.6.1. In this experiment, the instruments comprise: a pre-lab survey questionnaire (Section 3.6.2), UML system analysis documents (Section 3.6.3), UML system analysis documents with seeded defects (Section 3.6.4), questionnaires for the Comprehension and maintenance tasks (Section 3.6.5), and a post-lab survey questionnaire (Section 3.6.6).

\subsubsection{Preparation strategy}

All seeded defects, comprehension questions, and maintenance questions are derived in accordance with the following criteria:

- must cover different aspects/parts of the analyzed systems to the largest extent possible; 
- $\quad$ should not be trivial;

- should not be nearly impossible to address based on the available information in the allocated time;

- should be relevant to what software engineers would encounter in performing system analysis. For example, maintenance changes had to be plausible changes;

- should not relate to implementation details. For example, how to traverse a linked list is not a concern; and

- could be addressed with or without OCL in order to yield conservative results. Conservative results are desirable as they represent lower bounds of benefits brought by OCL. If there are some Comprehension and Maintenance questions that can only be answered in the presence of OCL expressions, obviously, then the performance for those who use OCL would be better, therefore biasing the results. Similarly, defects related to OCL syntax are not considered, as they cannot be detected from analysis documents without OCL. Thus, defects are inserted first into the UML-only version of the analysis documents, and then in the UML+OCL version, modifying OCL expressions where necessary. This ensures that the defects can be detected in both versions of the documents.

Standard techniques of phrasing subjective questions and designing survey questionnaires [15] are followed, so as to avoid bias and to increase reliability of responses: the questions should be simple, open / closing questions should be used appropriately, and answering choices should not overlap each other whenever a range is to be selected. 


\subsubsection{The pre-lab Survey}

A pre-lab survey questionnaire is administered to obtain information about the background of the subjects, e.g., their previous UML experience, the undergraduate program they belong to, etc. Information from the pre-lab survey can be used to verify if that the blocks used (Section 3.5.3.2) are appropriate.

\subsubsection{The UML system analysis documents}

There are two systems to be analyzed in this experiment: the Cab Distribution system (CD), and the Video Store system (VS). There are two versions for each of the system documents, one version includes OCL expressions, and the other version does not:

- correct CD with OCL (CD_OCL),

- correct CD without OCL (CD_no_OCL),

- correct VS with OCL(VS_OCL), and

- correct VS without OCL(VS_no_OCL).

All documents were reviewed in depth by the experimenters. Each document is about fourty pages long, as any larger document is believed to be unmanageable within the time constraints. The documents above are used for the Comprehension and the Maintenance tasks. 


\subsubsection{Documents with seeded defect}

Thirty defects are seeded in corresponding versions of each of the above system analysis documents for the Defect Detection task. The subjects are asked to identify all the seeded defects and submit the findings via e-mail. Answer sheets are not provided, as it may be difficult for the experimenter to read handwriting, which may cause unexpected misunderstanding. The subjects' performance is measured as the percentage of seeded defects correctly identified. The measurement is objective and repeatable. The system analysis documents with seeded detects include:

- CD with OCL and seeded defect (CD_E_OCL),

- CD without OCL but with seeded defect (CD_E_no_OCL),

- VS with OCL and seeded defect (VS_E_OCL), and

- VS without OCL but with seeded defect (VS_E_no_OCL).

Selected defects are seeded in class diagrams, data dictionaries, sequence diagrams, and statecharts. Different types of seeded defects usually encountered in software engineering are considered. We use the taxonomy shown in Table 11. 
Table 11. Type of defects

\begin{tabular}{|l|l|}
\hline & Type of defects \\
\hline Class diagram & Missing / wrong associations, such as wrong role name, wrong cardinality \\
\hline Data dictionary & $\begin{array}{l}\text { Inconsistencies with statecharts, such as missing states } \\
\text { Inconsistencies with class diagrams, such as wrong associations } \\
\text { Inconsistencies with sequence diagrams, such as wrong operation's } \\
\text { functionality }\end{array}$ \\
\hline Sequence diagram & $\begin{array}{l}\text { Missing / wrong sequences, such as missing operations } \\
\text { Missing / wrong guard conditions }\end{array}$ \\
\hline Statechart & $\begin{array}{l}\text { Missing / wrong states } \\
\text { Missing / wrong state transitions } \\
\text { Missing / wrong state transition conditions } \\
\text { Missing / wrong state transition actions }\end{array}$ \\
\hline
\end{tabular}

The complete list of the seeded defects can be found in Appendix A. Examples of defects seeded in the UML analysis document for the VS system are shown in Figure 3

Figure 3. Example defects seeded in the VS system analysis document

\section{Class Diagram}

Remove association constraint "Subset" between 2 associations connecting classes Reservation and Title

Data Dictionary

Operation PrebookedJob::verifyPreJob(): change description and return-list from "... confirmed job..." to " ... dispatched job..."

Sequence Diagram (Rent Video use case)

Remove condition "...(s2=1...)" from messages 1.2.11, 1.2.12, 1.2.13

StateChart (class Reservation)

Remove the transition from state "Pending" to "Cancelled"

All seeded defects can be detected based on the UML model alone, and do not require OCL, though OCL is expected to facilitate detection. The distribution of the seeded defects is similar in the two systems, as shown in Table 12. 
Table 12. Number of the seeded defects

\begin{tabular}{|c|c|c|}
\hline & CD & VS \\
\hline Class diagram & 5 & 5 \\
\hline Data dictionary & 10 & 10 \\
\hline Sequence diagram & 8 & 7 \\
\hline Statechart & 7 & 8 \\
\hline Total & 30 & 30 \\
\hline
\end{tabular}

\subsubsection{Questionnaires for the Comprehension and the Maintenance tasks}

During the Comprehension and the Maintenance tasks, the correct system analysis documents with OCL or without OCL are provided to the subjects. Questionnaires are distributed to ask specific questions about systems. All questions could be answered with or without OCL. Complete lists of the questions can be found in Appendix B.

\subsubsection{The Comprehension task questionnaire}

There are twenty questions for the Comprehension task for each analysis system. The questions are multiple choice. The subjects are asked to select the correct choice on the answer sheet. It is an easy way to quickly evaluate the extent to which a subject understands the system internal logic and functionality, which is measured as the percentage of all questions correctly answered. Another advantage of using multiple choice questions relates to ensuring that the evaluation would be objective and the measurement repeatable, as there is no room for interpreting which choice is correct for each question. Figure 4 shows an example of a multiple choice question used for the VS system. 
Figure 4. Example comprehension question
What is the OnHold
B. ForRent
C. OnHold or ForRent
D. Other answer:

\subsubsection{The Maintenance task questionnaire}

There are six and five prescribed system changes to the Cab Distribution system and the Video Store system respectively. The related questions focus on impact analysis. There are 71 model elements affected for the CD system, and 60 for the VS system. The subjects are asked to list model elements that would have to undergo change and submit their answers via e-mail (for the same reason discussed in Section 3.6.4). The subjects' performance is measured as the percentage of the total number of affected model elements that have been correctly identified. The measurement is objective and repeatable. Figure 5 shows an example of such a question for the VS system.

\section{Figure 5. Example Maintenance task question}

The video store company decides to limit the maximum number of unfulfilled reservations that a Member can perform in a day. Each member may have a different limit.

1. Which class(es) must be modified?

2. Which modifications (addition, change of attributes, operations, and/or operations' contracts) must be made in this (these) class(es)? 


\subsubsection{Post-lab surveys}

In addition, survey questionnaires are distributed after the execution of each laboratory to gain additional insight and help explain the results. The Comprehension and Maintenance tasks share the same survey questionnaire. Some of the questions apply to both tasks, while others are answered in the context of the specific task. The questionnaires focus on capturing the perception of the subjects regarding the tasks and the usefulness of OCL. The subjects are asked about whether they had enough time to perform the tasks, how easy the tasks were to perform, whether they understood the system's functionality, or whether the lab instructions were clear and easy to follow, etc. Moreover, when UML+OCL documents were used, the subjects were asked about the percentage of time spent reading and using OCL expressions, how easy the OCL expressions were to understand, and how helpful were they perceived to be.

Most questions are answered on a Likert scales [15]: the subjects are asked if, and to what extent, they agree or disagree with a statement: 1 being Strongly agree; 2 being Agree; 3 being Not certain; 4 being Disagree; and 5 being Strongly disagree. Complete survey question lists can be found in Appendix C. An example of a questionnaire for the Defect Detection task is shown in Figure 6. 
Figure 6. A survey questionnaire example

You are required to rate your level of agreement of the following statements. There are 5 levels of agreement:

1 - Strongly agree $\quad 2$ - Agree $\quad 3$ - Not certain $\quad 4$ - Disagree 5 - Strongly disagree

Please mark the corresponding box dark to provide your level of agreement of each statement.

1. I had enough time to read through the analysis document.

2. I had no problems to find errors in the document.

3. I would have found more errors if there would have been more time.

4. I fully understood what the system is trying to do.

5. I was not sure what type of errors I was looking for.

6. The system analysis was fairly complex.

7. The lab instruction is clear and easy to follow.

8. The OCL expressions in the document were easy to understand.

9. The OCL expression in the document helped me to find errors.

10.How much time did you spend in understanding the OCL expressions during this lab?
A. $<25 \%$
B. $>=25 \%$ and $<50 \%$
C. $>=50 \%$ and $<75 \%$
D. $>=75 \%$

The questionnaires are not part of the experiment proper, but rather gather information about the experiment. The output from the survey questionnaires is just meant to support and/or explain the quantitative results by providing qualitative insight.

\subsection{Analysis Procedure}

After each laboratory, both responses for the tasks and the survey questionnaire are collected and analyzed. Obtaining the experiment data is discussed in Section 3.7.1. A two-step experiment data analysis is described in Section 3.7.2. Section 3.7.3 provides an overview of how the survey data is analyzed. 


\subsubsection{Obtaining the experimental data}

Once the subjects performed the tasks, and submitted the answers, the correctness of each answer is evaluated. The measurement of each task is then put into spreadsheet for each subject. This measurement is defined to be as objective and repeatable as possible, as described in Sections 3.6.3 and 3.6.4.

\subsubsection{Analyzing the data}

A two-step data analysis is performed on subjects' performance data. The first step accounts for all the observations of both attempts at the tasks for each dependent variable (Section 3.7.2.1). However, because it is also necessary to consider possible ordering effects, which cannot be avoided, and to determine whether they interfere with the overall analysis (e.g., learning effect); whether the difference between UML+OCL and UMLOnly measurements can be explained by whether OCL is used during the first or the second attempt at a task must be tested. If this is the case, the data regarding each laboratory may have to be analyzed independently. Thus, a second analysis step is performed on each dependent variable for each laboratory (Section 3.7.2.2).

As discussed in Section 2.4, a number of assumptions have to be satisfied to enable statistical testing. A detailed analysis shows that differences in variance are not statistically significant, as shown in Appendix E. Departures from the normality assumption exist, but these are deemed to be very mild (when present), and the dependent 
variable distributions do not show extreme outliers or multi-modal distributions. The issue of departure from the assumptions can be addressed by having nearly equal samples sizes (Section 2.4). Differences in sample sizes exist, but these are rather small (see Tables in Chapter 4). The Wilcoxon test is also used as an alternative to the t-test, as the Wilcoxon test is resilient to strong departures from the t-test assumptions (as discussed Section 2.4.1). In this thesis, the results from the Wilcoxon test are only reported when they are different from the results from the t-test.

\subsubsection{Step 1: Analyzing data of both attempts at a task}

\section{All observations analysis}

As all the subjects perform the tasks twice, using and not using OCL, the one-tailed paired t-test [6] is performed for each task to test the corresponding hypothesis defined in Section 3.3 , using all the observations for that task. If the derived $p$-value is $\leq 0.05$, it can be concluded that using OCL has a positive effect on the corresponding dependent variable.

\section{Testing effect of Order of Method}

Half of the subjects apply the no_OCL treatment first and then the $O C L$ treatment (referred to as order N_O), while others apply these treatments in the reverse order (referred to as order O_N). If the effect of Order of Method exists, it would be necessary to study the effects of Method for each attempt at the tasks. 
In order to study the effect of Order of Method, the measurement differences between observations of the subjects using and not using OCL are calculated, formally: Diff $x=$

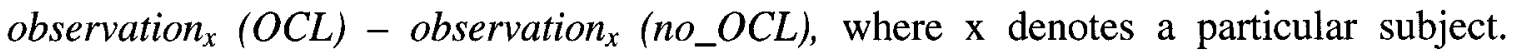
$\operatorname{Diff}\left(N_{-} O\right)$ denotes the performance difference (Diff) of the subjects who work on the system documents in the order of N_O, while Diff $\left(O \_N\right)$ denotes the performance difference of the subjects who work on the system documents in the order of O_N. The one-tailed t-test [6] is performed to test whether $\operatorname{Diff}\left(N_{-} O\right)$ is larger than Diff $\left(O \_N\right)$. The corresponding one-tailed Wilcoxon test is also performed. Diff $\left(N_{-} O\right)$ is expected to be larger than Diff( $\left.O_{-} N\right)$, as the subjects' ability to use OCL may improve through training received between laboratories, formally: $H_{0}: \operatorname{Diff}\left(N \_O\right)=\operatorname{Diff}\left(O \_N\right), H_{a}: \operatorname{Diff}\left(N \_O\right)>$ $\operatorname{Diff}\left(O \_N\right)$. A p-value of $\leq 0.05$ indicates that using OCL during the second attempt brings significantly larger performance difference between using and not using OCL than during the first attempt. (There are different ways of calculating the score difference between OCL and no_OCL groups. Statistics tests are also performed on Diff $f_{x}=$ Observation $_{x}(\operatorname{Ln}(O C L)) / \operatorname{Observation}_{x}(\operatorname{Ln}($ no_OCL $))$, which do not yield any difference.)

\section{Performance comparison between the first and the second attempt at a task}

In order to further understand the effect of Order of Method and the learning effect, the one-tailed paired t-tests are also performed to compare the subjects' performance between the first and the second attempt at a task. It is expected that the subjects' performance improves over time since the subjects are likely to gain experience from the 
first attempt at a task. A p-value of $\leq 0.05$ indicates that the subjects perform significantly better during the second attempt than during the first.

\subsubsection{Step 2: Data analysis per laboratory}

When ordering effects exist, it is necessary to look into the data one laboratory at a time. To study the effect of Method in each laboratory, a one-tailed $t$-test $[5,8]$ for the Method factor would do. The one-tailed t-test is then used to test the hypotheses defined in Section 3.3 for each laboratory. If the derived $p$-values are $\leq 0.05$, it can be concluded that using OCL has a positive effect on the dependent variables.

The effect of other factors may also be of interest. For example, the subjects' ability is likely to have impact on their performance. Further, Method may have a different effect (interaction) at different levels of subjects' ability. The interactions between factors can be studied with an Analysis of Variance (ANOVA). In this experiment, the effect of Ability, System and their interactions with Method are studied to refine the data analysis. To assure the robustness of ANOVA (Section 2.4.2), the factors are included in ANOVA in order of increasing importance, i.e., System being the first, Ability being the second, and Method being the last. Any $p$-value $\leq 0.05$ leads to the conclusion that the corresponding factor has a significant effect, or that it interacts with other factors significantly.

To study the effect of abovementioned three factors, three-way ANOVA should be used. Unfortunately, in the first experiment trial, the available data does not permit three-way 
ANOVA as the sample size is too small to yield valid results. Instead, two-way ANOVA is performed for Method \& Ability, and Method \& System, respectively. A three-way ANOVA is performed for Method, Ability and System in the second experiment trial, as the sample size is large enough (further discussed below).

As discussed in Section 2.4.2, ANOVA cannot be used to test which group performs better with respect to another, but can only tell whether groups have significantly different performances. A Descriptive statistics table and a Graph of means diagram are then used, in addition to the ANOVA table, to visually illustrate which group performs better.

Table 13 shows an example of a Descriptive statistics table. The rows of the table represent the two treatments of Method: OCL and no_OCL, while the columns of the table represent the two levels of Ability: High and Low. For each pair of two treatments (levels) of two different factors, such as High Ability and $O C L$, observation Mean values and Sample sizes are given. The last row represents the High and the Low Ability subjects' observations mean values and sample sizes without taking Method into account. Similarly, the last two columns represent the observation mean values and sample sizes for the subjects using OCL and not using OCL (no_OCL), respectively, without taking Ability into account. Corresponding Graphs of Means are then created to illustrate the observation means of each group for both factors, as shown in Figure 7. Non parallel mean value lines indicate possible interactions between the corresponding factors. For example, the two mean lines are crossing in Figure 7, suggesting that, when OCL is used, the high ability subjects perform better, while the low ability subjects perform worse. 
Table 13. Descriptive statistics (example)

\begin{tabular}{|c|c|c|c|c|c|c|}
\hline \multirow[b]{3}{*}{ Method } & \multicolumn{6}{|c|}{ Ability } \\
\hline & \multicolumn{2}{|c|}{ High } & \multicolumn{2}{|c|}{ Low } & \multicolumn{2}{|c|}{ All } \\
\hline & Mean & Size & Mean & Size & Mean & Size \\
\hline OCL & $11.43 \%$ & 7 & $4.07 \%$ & 9 & $7.3 \%$ & 16 \\
\hline No_OCL & $8.33 \%$ & 8 & $5.7 \%$ & 7 & $7.1 \%$ & 15 \\
\hline All Methods & $9.77 \%$ & 15 & $4.8 \%$ & 16 & $7.2 \%$ & 31 \\
\hline
\end{tabular}

Figure 7. Graph of means by Method and Ability (example)

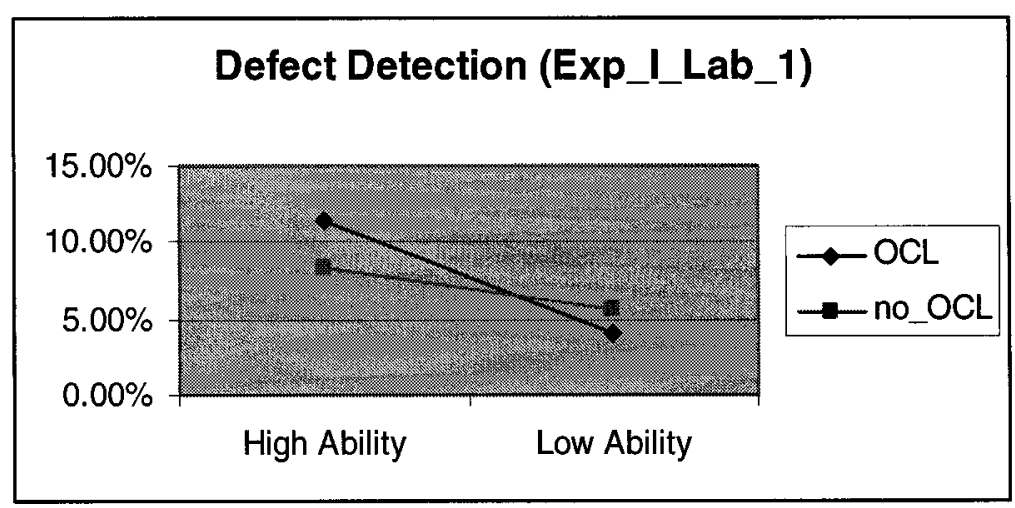

\subsubsection{Examining the survey data}

The $t$-test is used to examine the survey data. The t-test is one-tailed, as using OCL and the learning effect are expected to have effects in a specific direction (e.g., questions are easier to answer and therefore exhibit better scores with OCL). The equivalent one-tailed Wilcoxon test is also used in case the t-test statistical assumptions do not hold. Note that the two tests do not yield many differences in results.

When comparing differences in questionnaire data between subsequent laboratories, the paired t-test is used for paired samples to monitor the learning effect and explain 
differences in results that may be observed. The paired t-test is also one-tailed as the learning effect is expected over subsequent laboratories, leading to improvement in performance, and therefore to a change in questionnaire scores, e.g., a subject may find a task difficult the first time it is performed, but not any more the second time.

\subsection{Threats to validity}

Results from an experiment should be valid for the population of interest (Validity), which is a fundamental concern that has to be addressed in experimentation. There are four classification schemes for different types of threats to the validity of an experiment, in order of decreasing priority: internal, external, construct and conclusion validity [19, 22].

\subsubsection{Internal validity}

Internal validity concerns the threats that can affect the factors with respect to causality without the researcher's knowledge, e.g. subject's ability may jeopardize the experiment results if it is not controlled. Any confounding effects due to the system being used, the ability of the subjects, or the order of performing the tasks, are prevented. One possible issue relating to the experiment design (Table 8) is the possible information exchange among the subjects between the laboratories. This is prevented in several ways. First, the subjects are not aware of the detailed plan for the laboratories, and therefore do not know 
that they have to perform the same tasks as their fellow students of other groups at a later time. Further, the subjects do not have access to the lab material between laboratories (not to the system descriptions, nor to the questionnaires). Lastly, all laboratories are closely monitored, so that individuals' abnormal behavior can be observed and their data discarded if necessary.

Another concern relates to the fact that the subjects perform the three tasks on the same system document in two subsequent laboratories, some with OCL first, the others later (Table 8): for instance, Group 1 subjects perform the Defect Detection task on the Cab Distribution System with OCL in Laboratory 1, and the Comprehension and Maintenance tasks on the same system without OCL in Laboratory 2 . If the subjects remembered the OCL expressions from the first laboratory, it may help them to perform better in the following laboratory. A possible solution is to switch the documents used by Group 1 and Group 3 in Laboratory 2. If the threat is real, a performance difference should be observed between Group 1 and Group 2, as the former group uses two different systems, while the latter uses the same system in Laboratories 1 and 2. However, Group 1 and Group 3 will see both systems in the first two laboratories. Their levels of understanding of the systems may be different from their fellow subjects of Group 2 and Group 4 in the subsequent laboratories. Thus, such document assignment is not desirable. On the other hand, it is unlikely that the subjects would remember the OCL expressions from the first laboratory: the laboratories are one week apart in Experiment I, and two weeks apart in 
Experiment II; again the subjects do not know what to expect in the next laboratory, and have no access to lab material between the laboratories.

Another issue relating to internal validity is that the absence or presence of the subjects cannot be fully controlled, as the experiment is conducted in the context of a university course. Students may drop out of the course, or simply not show up. If the absent subjects are of a specific category, e.g., high ability subject, the similarity in the treatment groups' characteristic may not hold anymore. Fortunately, the grade distributions in the four laboratories are not very different from each other, as shown in Figure 8.

Figure 8. Grade distribution of Experiment I

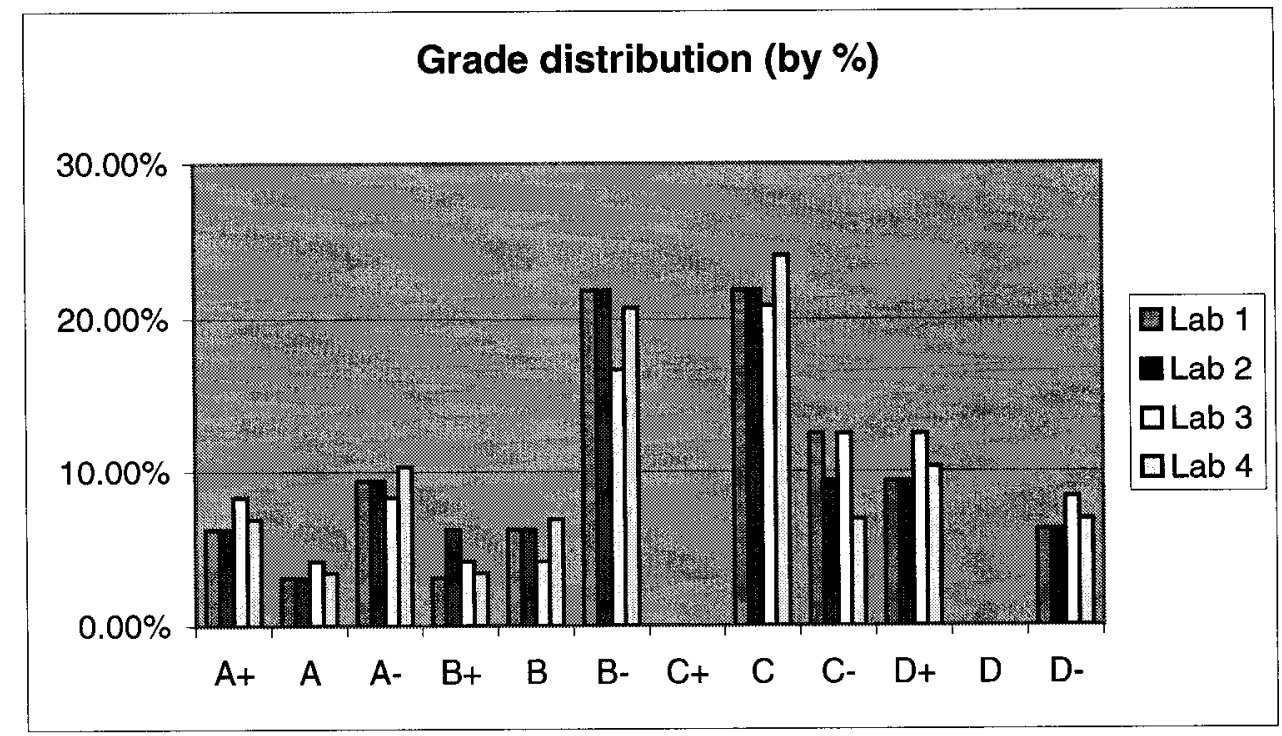

\subsubsection{External validity}

The main threats to validity in this experiment relate to external validity, which concerns generalizing the experiment results to a broader population. Like any academic 
experiments, the issue of whether the subjects are representative of software professionals arises. Recall that the $4^{\text {th }}$ year engineering students involved in this experiment are well trained at software modeling using UML and OCL. If there is an issue, it is not the lack of UML knowledge of the subjects, but rather that they represent the ideal, unusual case of subjects having been through a thorough UML training, but without having practiced their knowledge in an industrial environment. However, the difference between students and professionals is not always a clear cut, as reported in a recent experiment [1]: the performance of undergraduate and graduate students (whose expertise is similar to the expertise of the subjects in this experiment) at maintaining a UML design was not found to be very different from the performance of junior and intermediate professional consultants.

Another external validity issue, which is unfortunately inherent to controlled experiments, relates to the size and complexity of the system models, and concerns whether OCL would have an equal, weaker, or stronger impact if the system models had been vastly more complex. That question cannot be answered. The controlled experiment, such as the one presented here, is considered as a preliminary step in assessing whether actual case studies in industrial settings should even be considered.

\subsubsection{Construct validity}

Construct validity relates to the measurement instruments, including the defects seeded, the comprehension questionnaires, maintenance questionnaires, and the survey 
questionnaires. Threats to Construct validity is addressed in Section 3.6.1. The seeded defects are selected to achieve a balance: they have to be complex enough so as not to be obvious at a first sight; they also have to be detectable with or without OCL so as not to bias the results in favor of using OCL. The Maintenance and comprehension questions are also selected to be of certain level of complexity and to cover as many parts of the models as possible, while being answerable within the time available. During the survey, the following could have been a possible bias: when asked about their perception of the usefulness of OCL, the subjects could have answered what they thought was expected by the experimenters. Evidence shows this is, however, not the case, as discussed in Chapters 4.

\subsubsection{Conclusion validity}

Conclusion validity concerns issues that affect the ability to draw the correct conclusion about relations between the treatments and the outcome of an experiment. Conclusion validity relates to subject selection, data collection, measurement reliability, and the validity of the statistical tests, which are addressed in the design of the experiment (Sections 3.4 to 3.7). 


\subsection{Repeated experiment trial (Experiment II)}

A second experiment trial, Experiment II, is conducted, repeating the steps of the first experiment trial as an attempt to confirm its results. The same instruments are used, e.g., system analysis documents, questionnaires. There are a few differences in the second experiment trial: larger sample size, longer experiment duration, more prior training, and different task time allocation, as discussed in Section 3.9.1. Section 3.9.2 discusses the difference between the subjects of Experiment I and Experiment II, and how Blocking is applied in Experiment II. Section 3.9.3 discusses threats to validity that are unique to Experiment II.

\subsubsection{Differences in Experiment II}

\section{Sample size and experiment duration}

The number of subjects is doubled in Experiment II from Experiment I, to 89 in total, which enables performing a three-way ANOVA for the Method, Ability and System factors. Due to classroom space constraints, each group has to be further divided into two sub-groups, with each sub-group attending laboratory every two weeks. Experiment II covers a time period of eight weeks. 


\section{Prior training}

More training was administered before the second experiment trial than in the first trial. More examples were given to the subjects to familiarize themselves with UML system analysis documents, OCL expressions, and the tasks at hand.

\section{Task time allocation}

It was observed in Experiment I that, overall, the subjects did not use all the time allocated to perform the Comprehension task (90 minutes), while the subjects needed more time to perform the Maintenance task (60 minutes). Thus 75 minutes are allocated to each of the two tasks in Experiment II.

\subsubsection{Subjects' ability and Blocking}

\section{The subjects' ability}

There are differences in grade distributions between Experiment I and Experiment II, as shown in Figure 9. For instance, $6 \%$ of the subjects in Experiment I are A+ students, whereas they represent only $1 \%$ of the subjects in Experiment $\Pi$. The comparison of the two distributions suggests that the ability of the subjects participating in Experiment $\mathrm{I}$ is slightly better than that of those participating in Experiment II. 53\% of the subjects are categorized as "High ability" subjects (B- and above) in Experiment I, while only 45\% 
are in Experiment II. However, overall, Figure 9 also shows that the grade distributions are similar across the two experiment trials.

Figure 9. Grade distribution for Experiment I and II

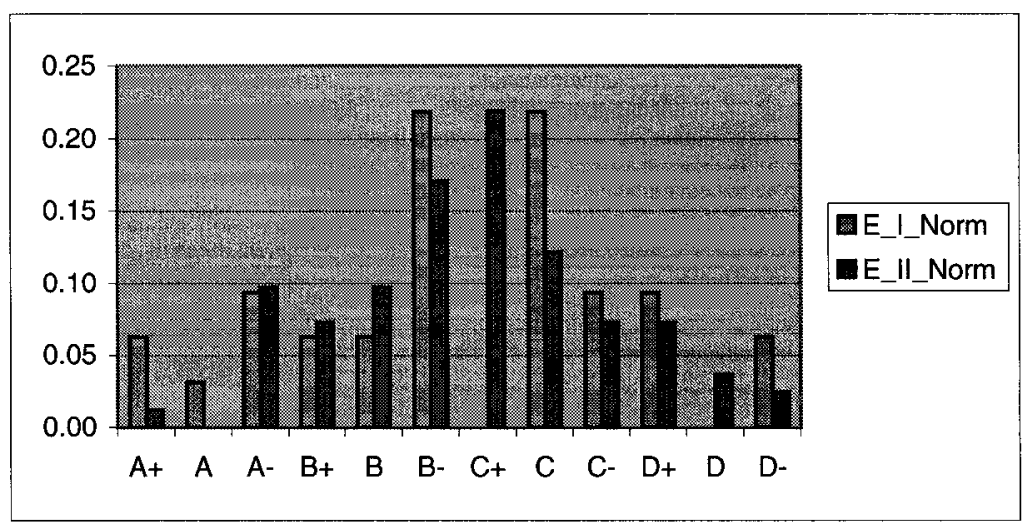

\section{Blocking}

There is only one Electrical Engineering student, thus the blocking for Academic Background is again not applied. The subjects are blocked according to their grades of the prerequisite course and their previous experience of OCL, as shown in Table 14. The threshold, which divides high and how ability subjects, remains at B- to be consistent with Experiment I. The sample sizes of groups are not equal, but the differences are small, as shown in Table 14. 
Table 14. Blocking in Experiment II

\begin{tabular}{|c|c|c|c|c|c|}
\hline & \multirow[b]{2}{*}{ Sub group } & \multicolumn{2}{|c|}{ OCL experience } & \multicolumn{2}{|c|}{ Ability } \\
\hline & & $\begin{array}{l}\text { Learned prior } \\
\text { to the course }\end{array}$ & $\begin{array}{l}\text { Learned in } \\
\text { the course }\end{array}$ & High & Low \\
\hline \multirow{4}{*}{ Group 1} & Group 1-1 & 1 & 10 & 5 & 6 \\
\hline & Group 1-2 & 0 & 10 & 4 & 6 \\
\hline & Group 1-3 & 1 & 9 & 5 & 5 \\
\hline & Group 1-4 & 0 & 11 & 5 & 6 \\
\hline \multirow{4}{*}{ Group 2} & Group 2-1 & 1 & 10 & 5 & 6 \\
\hline & Group 2-2 & 0 & 10 & 5 & 5 \\
\hline & Group 2-3 & 1 & 9 & 4 & 6 \\
\hline & Group 2-4 & 0 & 11 & 5 & 6 \\
\hline
\end{tabular}

\subsubsection{Threats to validity}

The differences between Experiment I and Experiment II introduce new threats to internal validity. For instance, since the subjects have to be divided into two sub-groups and perform the same tasks in two subsequent laboratories, it is possible that the subjects from the second sub-group may hear from the first sub-groups about the tasks and the analyzed systems. Cheating was actually observed in a few cases, as the students were monitored, and the corresponding data were discarded. Further, comparing the performance of the sub-group pairs, there are no significant differences in general. Furthermore, as discussed before, though the laboratories are compulsory, the students' attendance cannot be enforced. There are students who did not show up to some of the laboratories. However, the dropouts do not affect the grade distributions of the four laboratories much, as shown in Figure 10. Thus, internal validity still holds. 
Figure 10. Grade distribution for Experiment II

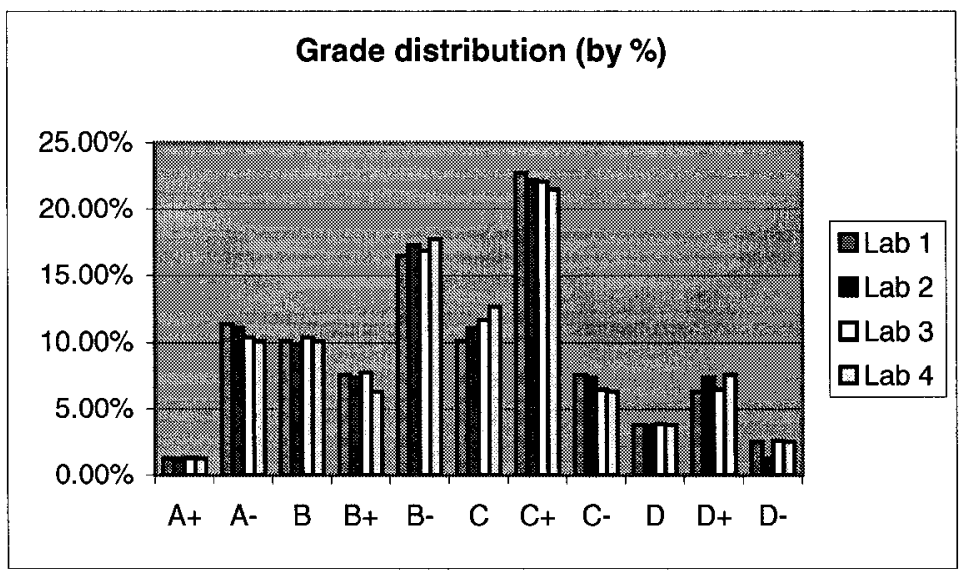




\title{
CHAPTER 4 THE EXPERIMENTAL RESULTS AND
}

\begin{abstract}
ANALYSIS
This chapter presents the results and the analysis for both experiment trials. Sections 4.1, 4.2, and 4.3 respectively provide the analysis results for the dependent variables Defect Detection, Comprehension and Maintenance. In each section, results of Experiment I, Experiment II, and their comparison, are reported. For each task in an experiment trial, the results from the two-step analysis (Section 3.7.2) are reported.
\end{abstract}

In the first step of data analysis, all observations for the two attempts at the tasks are analyzed together. The effect of Method is analyzed via the one-tailed paired t-test. The effect of Order of Method is analyzed via the one-tailed t-test. The performance difference between the two attempts at the tasks is analyzed via the one-tailed t-test as well.

In the second step of data analysis, the one-tailed t-test is used to analyze the effect of Method for each laboratory, followed by two two-way ANOVA (Method \& Ability, and Method \& System) results for Experiment I, and a three-way ANOVA (Method, Ability \& System) results for Experiment II. 
The supporting survey results are also presented. For brevity of the description presented herein, all survey results tables are captured in Appendix D. An overall discussion of results is presented in Section 4.4.

In order to simplify the presentation of the tasks performed during the different laboratories in the different experiment trials, the following convention is used: <Task>$<$ Lab $>-<$ Experiment trial $>$. For example, Comprehension-2-I $(C-2-I)$ refers to the Comprehension task performed during Laboratory 2 in Experiment I.

In order to simplify the presentation of the subjects, the following terms are used, as shown in Table 15:

Table 15. Types of subjects

\begin{tabular}{|l|l|}
\hline Type of subjects & Description \\
\hline OCL subjects & $\begin{array}{l}\text { The subjects who performed a task on UML+OCL version of system } \\
\text { analysis documents }\end{array}$ \\
\hline No_OCL subjects & $\begin{array}{l}\text { The subjects who performed a task on UML-only version of system analysis } \\
\text { documents }\end{array}$ \\
\hline N_O subjects & $\begin{array}{l}\text { The subjects who performed a task on UML-only version of a system } \\
\text { analysis document during the first attempt at the task, and on UML+OCL } \\
\text { version of the document during the second attempt }\end{array}$ \\
\hline O_N subjects & $\begin{array}{l}\text { The subjects who performed a task on UML+OCL version of a system } \\
\text { analysis document during the first attempt at the task, and on UML-only } \\
\text { version of the document during the second attempt }\end{array}$ \\
\hline
\end{tabular}




\subsection{Defect detection (D)}

\subsubsection{Experiment I}

\subsubsection{Analyzing data of both laboratories}

\section{All observations analysis}

$\mathrm{H}_{0}: \mathrm{D}(\mathrm{OCL})=\mathrm{D}($ no_OCL)

$\mathrm{H}_{\mathrm{a}}: \mathrm{D}(\mathrm{OCL})>\mathrm{D}($ no_OCL)

The one-tailed paired t-test is used to test the above hypothesis. Overall, the subjects perform slightly better when OCL is used than when OCL is not used, but the difference is not significant: the $t$-test does not yield any significant results for the Defect Detection task for Experiment I $(\mathrm{p}$-value $=0.302$, mean $(\mathrm{OCL})=11.67 \%$, mean (no_OCL) $=$ $10.28 \%$ ). Since the null hypothesis cannot be rejected at $\alpha=0.05$, there is no evidence that using OCL has a positive effect on the Defect Detection dependent variable in this experiment trial.

\section{Testing the effect of Order of Method}

$\mathrm{H}_{0}: \operatorname{Diff}\left(\mathrm{N} \_\mathrm{O}\right)=\operatorname{Diff}\left(\mathrm{O} \_\mathrm{N}\right)$

$\mathrm{H}_{\mathrm{a}}$ : Diff(N_O) $>$ Diff(O_N)

The one-tailed t-test is performed to determine if Order of Method matters (above hypothesis). The $t$-test yields a significant result: $\mathrm{p}$-value $=0.002$, which suggests that the order in which OCL is applied has a significant effect on the difference between the 
subjects' performance using and not using OCL. The N_O subjects perform better when they apply OCL compared to when they do not $\left(\right.$ mean $\left.\left(\operatorname{Diff}\left(\mathrm{N} \_\mathrm{O}\right)\right)=7.95 \%\right)$, while the O_N subjects perform worse $\left(\operatorname{mean}\left(\operatorname{Diff}\left(\mathrm{O} \_\mathrm{N}\right)\right)=-6.36 \%\right)$, as illustrated in Table 16 . It seems that using OCL has a negative effect during the first attempt at the task, but a positive effect during the second attempt. The two results cancel each other out, which leads to overall insignificant results.

However, the above interpretation may be short sighted as the learning effect is not considered. The learning effect may confound with the effect of using OCL. More precisely, the N_O subjects' performance difference between Laboratory 1 and Laboratory 3 may be due to the use of OCL (effect of Method), the learning effect, or both, denoted as: OCL effect + learning effect. On the other hand, if any performance improvement exists for the O_N subjects, the learning effect must be stronger than the possible OCL effect that may exist in Laboratory 1. The O_N subjects' performance difference between Laboratory 1 and Laboratory 3 can be denoted as learning effect OCL effect.

In accordance with this experiment, the O_N subjects' performance improves by $6.36 \%$ from Laboratory 1, where OCL is used, to Laboratory 3, where OCL is not used. It is possible that using OCL may have a negative impact causing the difference. It is also possible that the O_N subjects experience the learning effect only. Meanwhile, the N_O subjects' performance also improves from Laboratory 1 , where OCL is not used, to Laboratory 3, where OCL is used. The difference may be due to the positive effect of 
using OCL and/or the learning effect. The difference between the performance improvement of the N_O subjects and of the O_N subjects is rather small (1.59\%). Thus, it is likely that the N_O subjects' performance improvement over time is mainly due to the learning effect, but not much to the use of OCL. The existence of the learning effect finds support in the survey data. Table 83 on page 181 indicates that the subjects are significantly more confident in the type of defects to look for in Laboratory 3 than in Laboratory $1(p$-value $=0.027$, mean $(\mathrm{lab} 1)=2.96$, mean $(\mathrm{lab} 3)=3.4)$. Table 85 on page 181 shows that the subjects spend significantly more time in reading and using OCL in Laboratory 3 than in Laboratory 1.

Therefore, using OCL may not have any effect in both attempts at the task. Whether using OCL has opposite effects or no effect during the two attempts at the task can only be studied via the next step of data analysis, where observations from each attempt are analyzed respectively.

Table 16 Order of Methods applied

\begin{tabular}{|l|c|c|c|}
\hline & Lab 1 & Lab 3 & Diff(OCL - no_OCL) \\
\hline O_N & OCL & No_OCL & $-6.36 \%$ \\
\hline N_O & No_OCL & OCL & $7.95 \%$ \\
\hline
\end{tabular}

\section{Comparing performance between Laboratory 1 and Laboratory 3}

Although the absolute performance improvement of the subjects is small $(14.58 \%$ $7.36 \%=7.22 \%)$, the relative improvement is substantial $(98.1 \%)$ from Laboratory 1 to 
Laboratory 3, as shown in Table 17 . This improvement is statistically significant (p-value $=0.002)$, and it is particularly large for the low ability subjects $(109.94 \%)$.

Table 17. Improvement by Ability (D-1-I and D-3-I)

\begin{tabular}{|c|c|c|c|c|}
\hline & Mean (Laboratory 1) & Mean (Laboratory 3) & Improvement & p-value \\
\hline High Ability & $10 \%$ & $19.1 \%$ & $91 \%$ & \multirow{2}{*}{ - } \\
\cline { 1 - 4 } Low Ability & $5.13 \%$ & $10.77 \%$ & $109.94 \%$ & \\
\hline All subjects & $7.36 \%$ & $14.58 \%$ & $98.1 \%$ & $\mathbf{0 . 0 0 2}$ \\
\hline
\end{tabular}

Looking further into the observations, shown in Table 18, the only subjects that statistically improve their performance from Laboratory 1 to Laboratory 3 are the N_O low ability subjects (p-value $=0.019$ ). Further, the N_O low ability subjects have larger performance improvement $(9.07 \%)$ than the O_N low ability subjects $(1.67 \%)$. This suggests that, besides the learning effect, using OCL may have impact on the low subjects' performance during the second attempt at the task in this experiment trial.

Table 18. Comparing the same subjects (paired t-test)

\begin{tabular}{|c|c|c|c|c|}
\hline & Ability & Mean (Lab 1) & Mean (Lab 3) & p-value \\
\hline \multirow{2}{*}{ O_N } & High & $12.00 \%$ & $24.00 \%$ & 0.065 \\
\cline { 2 - 5 } & Low & $4.43 \%$ & $6.10 \%$ & 0.318 \\
\hline \multirow{2}{*}{ N_O } & High & $8.33 \%$ & $15.00 \%$ & 0.115 \\
\cline { 2 - 5 } & Low & $5.70 \%$ & $14.77 \%$ & $\mathbf{0 . 0 1 9}$ \\
\hline
\end{tabular}

The survey data also indicates a positive effect of using OCL on the low ability subjects: Table 86 on page 182 shows that the low ability subjects spend significantly more time in reading and using OCL in Laboratory 3 than in Laboratory 1 (p-value $=0.014$, mean $($ lab1) $=1.56$, mean $($ lab3 $)=2.67$, while the high ability subjects do not (not significant). Table 85 on page 182 also shows that the low ability subjects know what 
type of defect to look for only in Laboratory 3 , but not in Laboratory 1 (p-value $=0.008$, mean $(l a b 1)=2.64$, mean $(l a b 3)=3.5)$, whereas the high ability subjects always know what type of defects to look for (mean $=\sim 3.3$ in both labs). This further suggests that using OCL may have helped the low ability subjects to perform the task in Laboratory 3, which results in their performance improvement over time. ANOVA is then performed for each attempt at the task to further investigate the interaction between the subjects' ability and using OCL (discussed below).

\subsubsection{Defect Detection-1-I}

\section{Testing the hypothesis- D-1-I}

$\mathrm{H}_{0}: \mathrm{D}(\mathrm{OCL})=\mathrm{D}($ no_OCL)

$\mathrm{H}_{\mathrm{a}}: \mathrm{D}(\mathrm{OCL})>\mathrm{D}$ (no_OCL)

The $t$-test yields a p-value of $0.47($ mean $(\mathrm{OCL})=7.3 \%$, mean(no_OCL) $=7.1 \%)$. Although the OCL subjects perform slightly better than the no_OCL subjects, since the null hypothesis cannot be rejected at $\alpha=0.05$, there is no evidence that a difference exists between using and not using OCL in this laboratory. This also suggests that using OCL does not have any negative effect on the subjects' performance at all.

\section{Two-way ANOVA (Method \& Ability) - D-1-I}

In Laboratory 1, only Ability has a statistically significant effect $(\mathrm{p}$-value $=0.037)$ on the percentage of defects detected, as shown in Table 19, explaining $15 \%$ of the total variation $\left(E^{2}=0.15\right)$. Table 20 and Figure 11 show that the high ability subjects perform better than the low ability subjects, irrespective of the methods used. The use of OCL, 
Method, does not have a significant effect ( $\mathrm{p}$-value $=0.752$ ). Although Figure 11 seems to suggest an interaction between Method \& Ability, it is not found to be significant (pvalue $=0.308)$.

Table 19. Two-way ANOVA (Method \& Ability) - D-1-I

\begin{tabular}{|c|c|c|c|c|c|c|}
\hline Source & DF & Sum of Squares & Mean Square & F Ratio & Prob $>$ F & $E^{2}$ \\
\hline Ability & 1 & 0.01907212 & 0.01907212 & 4.7933 & $\mathbf{0 . 0 3 7}$ & 0.15 \\
\hline Method & 1 & 0.00040586 & 0.00040586 & 0.102 & 0.752 & 0.003 \\
\hline Ability*Method & 1 & 0.00429531 & 0.00429531 & 1.0795 & 0.308 & 0.03 \\
\hline Residual & 27 & 0.10742956 & 0.003979 & & & 0.82 \\
\hline Total & 30 & 0.13132095 & & & & \\
\hline
\end{tabular}

Table 20. Descriptive statistics (Method \& Ability) - D-1-I

\begin{tabular}{|c|c|c|c|c|c|c|}
\hline \multirow[b]{3}{*}{ Method } & \multicolumn{6}{|c|}{ Ability } \\
\hline & \multicolumn{2}{|c|}{ High } & \multicolumn{2}{|c|}{ Low } & \multicolumn{2}{|c|}{ All } \\
\hline & Mean & Size & Mean & Size & Mean & Size \\
\hline OCL & $11.43 \%$ & 7 & $4.07 \%$ & 9 & $7.3 \%$ & 16 \\
\hline No_OCL & $8.33 \%$ & 8 & $5.7 \%$ & 7 & $7.1 \%$ & 15 \\
\hline All Methods & $9.77 \%$ & 15 & $4.8 \%$ & 16 & $7.2 \%$ & 31 \\
\hline
\end{tabular}

Figure 11. Graph of means by Method and Ability - D-1-I

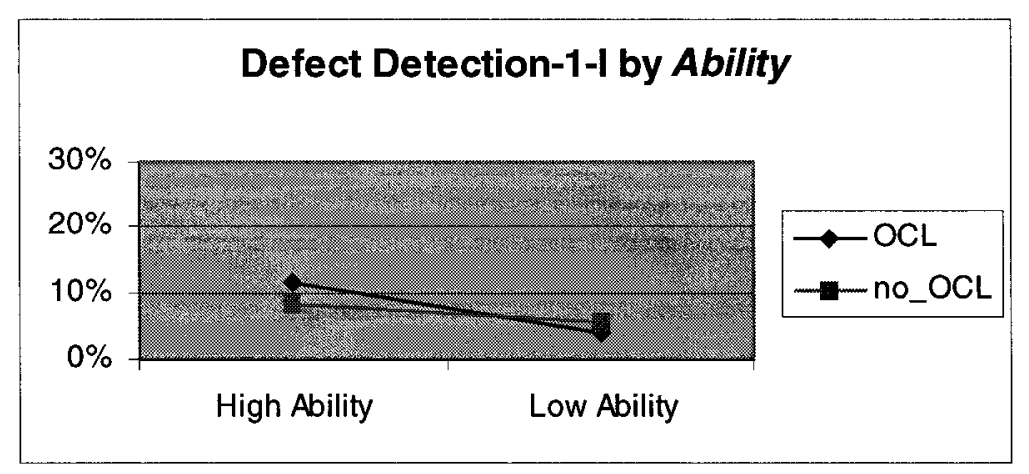

Two-way ANOVA (Method \& System) - D-1-I

Although Figure 12 and Table 22 show that the subjects working on the Cab Distribution system perform slightly better than the subjects working on the Video Store system, 
System does not have any significant main effect ( $\mathrm{p}$-value $=0.298$ ), as shown in Table 21 .

Neither the effect of Method (p-value $=0.827$ ), nor the interaction between Method and System $(\mathrm{p}$-value $=0.751)$ is found to be significant.

Table 21. Two-way ANOVA (Method \& System) - D-1-I

\begin{tabular}{|c|c|c|c|c|c|c|}
\hline Source & DF & Sum of Squares & Mean Square & F Ratio & Prob $>$ F & $E^{2}$ \\
\hline System & 1 & 0.0052359 & 0.0052359 & 1.1258 & 0.298 & 0.04 \\
\hline Method & 1 & 0.00022625 & 0.00022625 & 0.0486 & 0.827 & 0.002 \\
\hline System*Method & 1 & 0.00047996 & 0.00047996 & 0.1032 & 0.751 & 0.004 \\
\hline Residual & 27 & 0.12557276 & 0.004651 & & & 0.96 \\
\hline Total & 30 & 0.13132095 & & & & \\
\hline
\end{tabular}

Table 22. Descriptive statistics (Method \& System) - D-1-I

\begin{tabular}{|c|c|c|c|c|c|c|}
\hline \multirow[b]{3}{*}{ Method } & \multicolumn{6}{|c|}{ System } \\
\hline & \multicolumn{2}{|c|}{$\mathrm{CD}$} & \multicolumn{2}{|c|}{ VS } & \multicolumn{2}{|c|}{ All } \\
\hline & Mean & Size & Mean & Size & Mean & Size \\
\hline OCL & $9.43 \%$ & 6 & $6 \%$ & 10 & $7.3 \%$ & 16 \\
\hline No_OCL & $8.1 \%$ & 7 & $6.27 \%$ & 8 & $7.1 \%$ & 15 \\
\hline All Methods & $8.73 \%$ & 13 & $6.1 \%$ & 18 & $7.2 \%$ & 31 \\
\hline
\end{tabular}

Figure 12. Graph of Means by Method and System - D-1-I

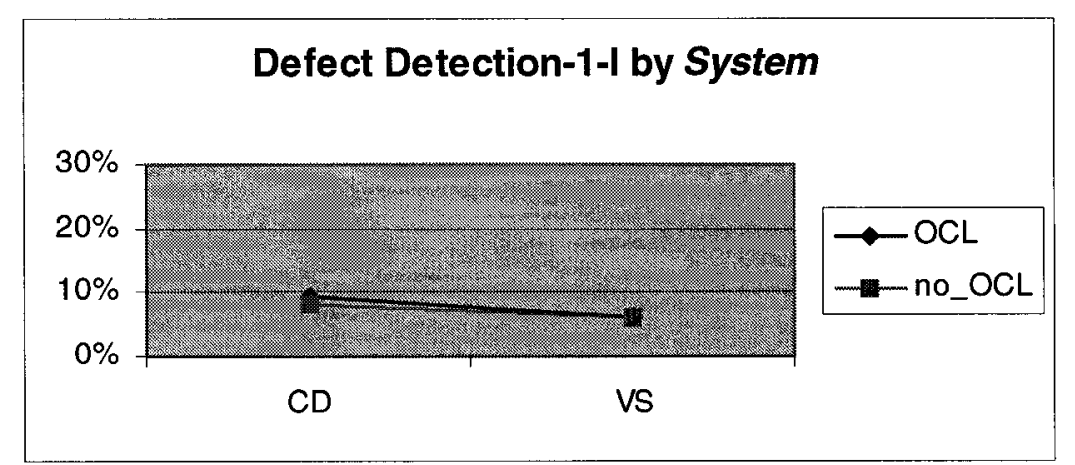


Combining both two-way ANOVA results - D-1-I

Combining the results of both two-way ANOVA tests, Ability is the only factor that has a significant main effect on Defect Detection in Laboratory 1 of Experiment I. Neither Method, nor System has any significant main effect on the subjects' performance. There are also no significant interactions among the factors.

However, the survey results do not support Ability having a significant main effect: there is no significant difference between the results of the high and the low ability subjects, as shown in Table 80 on page 180.

\subsubsection{Defect Detection-3-I}

\section{Testing the hypothesis- D-3-I}

$\mathrm{H}_{0}: \mathrm{D}(\mathrm{OCL})=\mathrm{D}($ no_OCL)

$\mathrm{H}_{\mathrm{a}}: \mathrm{D}(\mathrm{OCL})>\mathrm{D}($ no_OCL)

The $t$-test yields a $\mathrm{p}$-value of $0.442(\operatorname{mean}(\mathrm{OCL})=14.87 \%$, mean $($ no_OCL $)=14.23 \%)$.

Since the null hypothesis cannot be rejected at $\alpha=0.05$, it can be concluded that there is no significant difference between using and not using OCL in this laboratory. Using OCL does not have a positive or a negative effect.

Two-way ANOVA (Method \& Ability) - D-3-I

Table 23 shows that Ability has a significant main effect ( $\mathrm{p}$-value $=0.021)$, explaining $20 \%$ of the total variation $\left(E^{2}=0.2\right)$. Table 24 and Figure 13 show that the high ability subjects perform better than the low ability subjects overall. Method does not have a 
significant main effect ( $\mathrm{p}$-value $=0.962$ ), but has a significant interaction effect with Ability (p-value $=0.024)$ : Using OCL seems to have a negative effect on the high ability subjects, but a positive effect on the low ability subjects. This interaction explains $19 \%$ of the total variation $\left(\mathrm{E}^{2}=0.19\right)$.

Table 23. Two-way ANOVA (Method \& Ability) - D-3-I

\begin{tabular}{|c|c|c|c|c|c|c|}
\hline Source & DF & Sum of Squares & Mean Square & F Ratio & Prob $>$ F & $E^{2}$ \\
\hline Ability & 1 & 0.04858585 & 0.04858585 & 6.2976 & $\mathbf{0 . 0 2 1}$ & 0.20 \\
\hline Method & 1 & 0.00001799 & 0.00001799 & 0.0023 & 0.962 & 0.0001 \\
\hline Ability*Method & 1 & 0.04607644 & 0.04607644 & 5.9723 & $\mathbf{0 . 0 2 4}$ & 0.19 \\
\hline Residual & 20 & 0.15430028 & 0.007715 & & & 0.64 \\
\hline Total & 23 & 0.2418253 & & & & \\
\hline
\end{tabular}

Table 24. Descriptive statistics (Method \& Ability) - D-3-I

\begin{tabular}{|c|c|c|c|c|c|c|}
\cline { 2 - 8 } \multicolumn{1}{c|}{} & \multicolumn{5}{c|}{ Ability } & \multicolumn{2}{c|}{ All } \\
\cline { 2 - 8 } \multicolumn{1}{c|}{ High } & \multicolumn{2}{c|}{ Low } & Mean & Size & Mean & Size \\
\hline Method & Mean & Size & Meany & 13 \\
\hline OCL & $15 \%$ & 6 & $14.77 \%$ & 7 & $14.87 \%$ & 13 \\
\hline No_OCL & $24 \%$ & 5 & $6.1 \%$ & 6 & $14.23 \%$ & 11 \\
\hline All Methods & $19.1 \%$ & 11 & $10.77 \%$ & 13 & $14.6 \%$ & 24 \\
\hline
\end{tabular}

Figure 13. Graph of means (Method \& Ability) - D-3-I

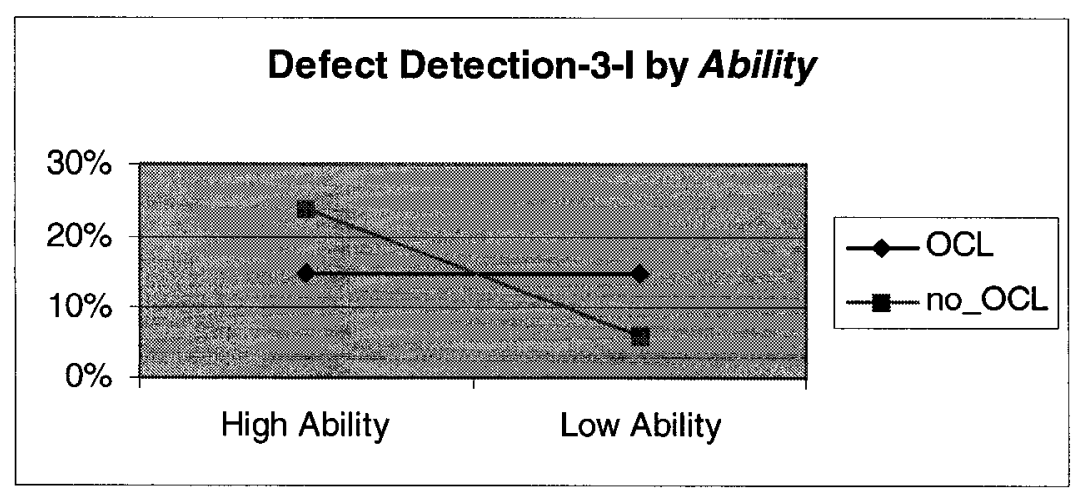


Two-way ANOVA (Method \& System) - D-3-I

Table 25 shows that System has a significant main effect (p-value $=0.008$ ), explaining $31 \%$ of the total variation $\left(E^{2}=0.31\right)$. Table 26 and Figure 14 show that the subjects perform better for the Cab Distribution system than for the Video Store system, irrespective of the methods used. Method, again, does not seem to have a significant effect. There is no significant interaction between Method and System, which is also apparent from Figure 14 and Table 26.

Table 25. Two-way ANOVA (Method \& System) - D-3-I

\begin{tabular}{|c|c|c|c|c|c|c|}
\hline Source & DF & Sum of Squares & Mean Square & F Ratio & Prob > F & $E^{2}$ \\
\hline System & 1 & 0.07378273 & 0.07378273 & 8.7972 & $\mathbf{0 . 0 0 8}$ & 0.31 \\
\hline Method & 1 & 0.00014799 & 0.00014799 & 0.0176 & 0.896 & 0.0006 \\
\hline System*Method & 1 & 0.0002212 & 0.0002212 & 0.0264 & 0.873 & 0.0009 \\
\hline Residual & 20 & 0.16774061 & 0.008387 & & & 0.69 \\
\hline Total & 23 & 0.2418253 & & & & \\
\hline
\end{tabular}

Table 26. Descriptive Statistics (Method \& System) - D-3-I

\begin{tabular}{|c|c|c|c|c|c|c|}
\cline { 2 - 7 } \multicolumn{1}{c|}{} & \multicolumn{4}{c|}{ System } & \multicolumn{2}{c|}{ All } \\
\cline { 2 - 8 } \multicolumn{1}{c|}{ CD } & \multicolumn{2}{c|}{ VS } & Mean & Size \\
\hline Method & Mean & Size & Mean & Size & M & \\
\hline OCL & $20.57 \%$ & 6 & $10 \%$ & 7 & $14.87 \%$ & 13 \\
\hline No_OCL & $20.67 \%$ & 5 & $8.9 \%$ & 6 & $14.23 \%$ & 11 \\
\hline All Methods & $20.6 \%$ & 11 & $9.5 \%$ & 13 & $14.6 \%$ & 24 \\
\hline
\end{tabular}


Figure 14. Graph of Means (Method \& System) - D-3-I

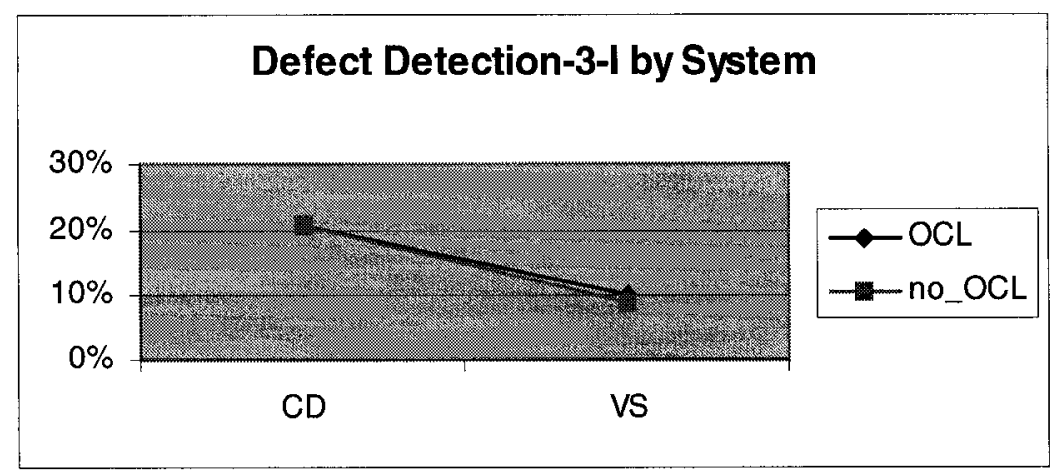

Combining both two-way ANOVA results - D-3-I

Combining both two-way ANOVA results in Laboratory 3, both Ability and System have significant main effects, while Method does not impact the subjects' performance significantly overall. However, Method has a significant interaction effect with Ability in Laboratory 3 representing $19 \%$ of the total variation $\left(E^{2}=0.19\right)$. The high ability subjects seem to do better without OCL, while the low ability subjects seem to do better with OCL.

The above interaction may be explained as follows: On one hand, OCL may have helped the low ability subjects to find more defects in a mechanical way. For example, by comparing statecharts and class invariants, inconsistencies between valid states can be observed without understanding what they really are. On the other hand, using OCL may have hindered the high ability subjects who have a more intuitive way to perform the task, based on a good understanding of object-oriented modeling, and who may be slowed down by trying to understand the OCL expressions. 
However, the survey results of Laboratory 3 do not support the above interpretation. Table 81 on page 180 shows that the low ability subjects who use OCL actually consider the systems to be relatively complex, compared to those who do not (mean(OCL)=2.14 vs. mean $($ no_OCL $)=3.29, \mathrm{p}$-value $=0.028)$. OCL does not seem to help the low ability subjects. On the other hand, the high ability subjects who use OCL have more confidence regarding the type of defects to look for, compared to those who do not $(\operatorname{mean}(\mathrm{OCL})=3.83$, mean $($ no_OCL $)=2.6, \mathrm{p}$-value $=0.030)$. This is inconsistent with the observed interaction between Method and Ability, and requires further investigation in further experiments.

Results from the survey questionnaire confirm the significant main effect of System. Table 83 on page 181 shows that, in Laboratory 3, the subjects working on the Cab Distribution system consider that they have enough time to finish the task, whereas the subjects working on the Video Store system do not $($ mean $(C D)=2.38$, mean $(V S)=3.39$, p-value $=0.028$ ). The subjects also consider the Video Store system to be more complex than the Cab Distribution system $($ mean $(V S)=2.33$, mean $(V D)=2.92$, p-value $(t$-test $)=$ 0.064, p-value (Wilcoxon test $)=0.053)$. However, the subjects consider the OCL expressions easy to understand for the Video Store system but not for the Cab Distribution system $($ mean $(C D)=3.5$, mean $(V S)=2.4$, p-value $=0.018)$. It is possible that OCL may add complexity to (considered) simpler systems, while making more complex systems easier to understand. This suggests an interaction between Method and System, which is not seen in the two-way ANOVA results of Experiment $\mathrm{I}$. 


\subsubsection{D-1-I and D-3-I analysis results discussion}

\section{Main effects}

Method does not have a significant main effect in either Laboratory 1 or Laboratory 3. This confirms that the subjects' performance improvement over time is likely due to the learning effect. It is possible that using OCL may not have any positive effect on a task like Defect Detection. However, further investigation shows that this is not the case as discussed below.

Ability has a significant main effect in both laboratories. The performance of the Defect Detection task seems to be determined by the ability of the subjects, regardless of which System is used or which Method is applied. System has a significant main effect in Laboratory 3 only, in which the subjects perform better for the Cab Distribution system than for the Video Store system. It is possible that the learning effect has a larger impact on simpler systems than on more complex systems.

\section{Interaction effects}

There is no significant interaction effect among the factors in Laboratory 1 . In Laboratory 3, the interaction effect between Method and Ability is significant. Using OCL seems to have a negative effect on the high ability subjects, but a positive effect on the low ability subjects. However, only the positive effect on the low ability subjects is statistically significant. More precisely, only the performance of the N_O low ability subjects' improves significantly from Laboratory 1 to Laboratory 3. 
However, there is an inconsistency in the survey results, which shows that the high ability subjects perceive to benefit from using OCL, whereas the low ability subjects do not. This inconsistency is difficult to explain at this point. More investigation is required to study the interaction between Method and Ability.

\subsubsection{Experiment II}

\subsubsection{Analyzing data of both laboratories}

\section{All observations analysis}

$\mathrm{H}_{0}: \mathrm{D}(\mathrm{OCL})=\mathrm{D}($ no_OCL $)$

$\mathrm{H}_{\mathrm{a}}: \mathrm{D}(\mathrm{OCL})>\mathrm{D}($ no_OCL $)$

A one-tailed paired t-test is used to test the above hypothesis, which shows that the performance of the subjects is significantly better when OCL is used compared to when OCL is not used for the Defect Detection task in Experiment II (p-value $=0.05$, mean $(\mathrm{OCL})=9.48 \%$, mean $($ no_OCL $)=7.76 \%$ ). Since the null hypothesis is rejected at $\alpha=0.05$, it can be concluded that using OCL has a positive effect on the dependent variable Defect Detection in this experiment trial.

\section{Testing effect of Order of Method}

$\mathrm{H}_{0}: \operatorname{Diff}\left(\mathrm{N} \_\mathrm{O}\right)=\operatorname{Diff}\left(\mathrm{O} \_\mathrm{N}\right)$

$\mathrm{H}_{\mathrm{a}}: \operatorname{Diff}\left(\mathrm{N} \_\mathrm{O}\right)>\operatorname{Diff}\left(\mathrm{O} \_\mathrm{N}\right)$

The one-tailed t-test is used to test the above hypothesis. The N_O subjects perform better when they use OCL compared to when they do not $\left(\operatorname{mean}\left(\operatorname{Diff}\left(\mathrm{N}_{-} \mathrm{O}\right)\right)=3.53 \%\right)$, 
while the O_N subjects perform slightly better $\left(\operatorname{mean}\left(\operatorname{Diff}\left(\mathrm{O}_{-} \mathrm{N}\right)\right)=0.09 \%\right)$, as shown in Table 27. The difference is significant ( $\mathrm{p}$-value $=0.05$ ). This suggests that the Order of Method has a confounding effect with Method: the subjects may have only been helped when they use OCL during the second attempt at the task.

The improvement of the O_N subjects' performance from Laboratory 1 to Laboratory 3 is almost negligible $(0.09 \%)$, which indicates that the learning effect may have overcome the positive effect of using OCL, if there is any. For the N_O subjects, the performance improvement $(3.53 \%)$ is likely due to the positive effect of using OCL, or to both the effect of using OCL and the learning effect. Whether or not a positive effect of using OCL exists in the second attempt at the task, but not in the first one, can only be confirmed in the second step of the data analysis, where the observations for each attempt are analyzed respectively.

Table 27 Order of Methods applied

\begin{tabular}{|c|c|c|c|}
\hline & Lab 1 & Lab 3 & Diff (OCL - no_OCL) \\
\hline O_N & OCL & No_OCL & $0.09 \%$ \\
\hline N_O & No_OCL & OCL & $3.53 \%$ \\
\hline
\end{tabular}

\section{Comparing performance between Laboratory 1 and Laboratory 3}

Although the subjects' performance improvement from Laboratory 1 to Laboratory 3 is not statistically significant $(\mathrm{p}$-value $=0.064$, mean $(\mathrm{lab} 1)=7.8 \%$, mean $(\mathrm{lab} 3)=9.42 \%$ ) overall, the performance of the N_O high ability subjects significantly improves over time, as shown in Table 28. This indicates that using OCL may have only helped the high 
ability subjects in the second attempt at the task. This can be further studied during the ANOVA analysis for each laboratory as discussed below.

The survey results suggest that the subjects' UML understanding is enhanced from Laboratory 1 to Laboratory 3 in this experiment trial. Table 92 on page 184 shows that the subjects are much more comfortable with the task and the systems in Laboratory 3 than in Laboratory 1: they feel less time pressure ( $\mathrm{p}$-value $<0.0001$, mean $(\mathrm{lab} 1)=3.27$, mean $($ lab3 $)=2.08$ ); have more confidence of the type of defects to look for ( $\mathrm{p}$-value = 0.031 , mean $(\operatorname{lab} 1)=2.75$, mean $(\mathrm{lab} 3)=3.02)$; have less problems to find defects $(\mathrm{p}-$ value $<0.0001$, mean $(\mathrm{lab} 1)=3.2$, mean $(\mathrm{lab} 3)=2.68)$; and perceive the system analysis to be less complex $(p$-value $=0.011$, mean $(\operatorname{lab} 1)=2.46$, mean $(\operatorname{lab} 3)=2.81$ ). Further analyzing the survey data, Table 96 on page 185 shows that only the high ability subjects consider OCL to be more helpful in Laboratory 3 than in Laboratory 1 ( $p$-value $=0.029$, mean $(\operatorname{lab} 1)=3.31$, mean $(\operatorname{lab} 3)=2.56)$, which supports that using OCL may have impact on the high ability subjects only.

Table 28. Comparing the same subjects (paired t-test)

\begin{tabular}{|c|c|c|c|c|}
\hline & Ability & Mean (Lab 1) & Mean (Lab 3) & p-value \\
\hline \multirow{2}{*}{ O_N } & High & $8.97 \%$ & $7.30 \%$ & 0.819 \\
\cline { 2 - 5 } & Low & $5.87 \%$ & $6.98 \%$ & 0.315 \\
\hline \multirow{2}{*}{ N_O } & High & $9.60 \%$ & $15.50 \%$ & $\mathbf{0 . 0 0 5}$ \\
\cline { 2 - 5 } & Low & $7.30 \%$ & $8.33 \%$ & 0.29 \\
\hline
\end{tabular}




\subsubsection{Defect Detection-1-II}

\section{Testing the hypothesis- D-1-II}

$\mathrm{H}_{0}: \mathrm{D}(\mathrm{OCL})=\mathrm{D}($ no_OCL $)$

$\mathrm{H}_{\mathrm{a}}: \mathrm{D}(\mathrm{OCL})>\mathrm{D}($ no_OCL)

The $t$-test yields a p-value of $0.661(\operatorname{mean}(\mathrm{OCL})=7.5 \%$, mean $($ no_OCL $)=8.13 \%)$. Since the null hypothesis cannot be rejected at $\alpha=0.05$, there is no difference between using and not using OCL in this laboratory. Even though the OCL subjects perform slightly worse than the no_OCL subjects, there is no evidence that using OCL has a negative effect either (corresponding $\mathrm{p}$-value $=0.339$ ).

\section{Three-way ANOVA (Method, Ability \& System) - D-1-II}

The three-way ANOVA results (Table 29) show that both Ability (p-value $=0.030$ ) and System $(\mathrm{p}$-value $=0.004)$ have significant main effects, while $\operatorname{Method}(\mathrm{p}$-value $=0.764)$ does not, in Laboratory 1 of Experiment II. System explains $11 \%$ of the total variation $\left(\mathrm{E}^{2}\right.$ $=0.11)$, while Ability explains $6 \%$ of the total variation $\left(E^{2}=0.06\right)$. The effect of Ability can be observed in Table 30 and Figure 15: the high ability subjects perform better than the low ability subjects for both systems. The effect of System is shown in Table 30 and Figure 16: the performance for the Cab Distribution system is better than that for the Video Store system, for both high and low ability subjects. There seems to be a mild interaction between Method and System (Figure 16), but it is not significant (p-value = 0.122 ). There is no significant interaction among the factors. 
Table 29. Three-way ANOVA (Method, Ability \& System)- D-1-II

\begin{tabular}{|c|c|c|c|c|c|c|}
\hline Source & DF & Sum of Squares & Mean Square & F Ratio & Prob $>$ F & $E^{2}$ \\
\hline System & 1 & 0.03641153 & 0.03641153 & 9.1445 & $\mathbf{0 . 0 0 4}$ & 0.11 \\
\hline Ability & 1 & 0.01950066 & 0.01950066 & 4.8975 & $\mathbf{0 . 0 3}$ & 0.06 \\
\hline Method & 1 & 0.00036177 & 0.00036177 & 0.0909 & 0.764 & 0.001 \\
\hline Ability*System & 1 & 0.00069328 & 0.00069328 & 0.1741 & 0.678 & 0.002 \\
\hline Method*System & 1 & 0.00974264 & 0.00974264 & 2.4468 & 0.122 & 0.03 \\
\hline Ability*Method & 1 & 0.00186359 & 0.00186359 & 0.468 & 0.496 & 0.006 \\
\hline Ability*Method *System & 1 & 0.00069201 & 0.00069201 & 0.1738 & 0.678 & 0.002 \\
\hline Residual & 68 & 0.27076071 & 0.003982 & & & 0.8 \\
\hline Total & 75 & 0.339007 & & & & \\
\hline
\end{tabular}

Table 30. Descriptive statistics (Method \& Ability) - D-1-II

\begin{tabular}{|c|c|c|c|c|}
\hline Method & Ability & System & Mean & Size \\
\hline \multirow{4}{*}{$\begin{array}{l}\text { OCL } \\
\text { Mean: } 7.5 \% \\
\text { Size: } 40\end{array}$} & \multirow{2}{*}{$\begin{array}{l}\text { High } \\
\text { Mean: } 9.8 \%, \text { Size: } 18\end{array}$} & $\mathrm{CD}$ & $11.48 \%$ & 9 \\
\hline & & VS & $8.15 \%$ & 9 \\
\hline & \multirow{2}{*}{$\begin{array}{l}\text { Low } \\
\text { Mean: } 5.6 \%, \text { Size } 22 \\
\end{array}$} & $\mathrm{CD}$ & $6.06 \%$ & 11 \\
\hline & & VS & $5.15 \%$ & 11 \\
\hline \multirow{4}{*}{$\begin{array}{l}\text { no_OCL } \\
\text { Mean: } 8.13 \% \\
\text { Size: } 36\end{array}$} & \multirow{2}{*}{$\begin{array}{l}\text { High } \\
\text { Mean: } 9.27 \%, \text { Size: } 18\end{array}$} & $\mathrm{CD}$ & $12.59 \%$ & 9 \\
\hline & & VS & $5.92 \%$ & 9 \\
\hline & \multirow{2}{*}{$\begin{array}{l}\text { Low } \\
\text { Mean: } 7.03 \%, \text { Size: } 18\end{array}$} & $\mathrm{CD}$ & $10.37 \%$ & 9 \\
\hline & & VS & $3.70 \%$ & 9 \\
\hline
\end{tabular}

Figure 15. Graph of Means by Method and Ability - D-1-II
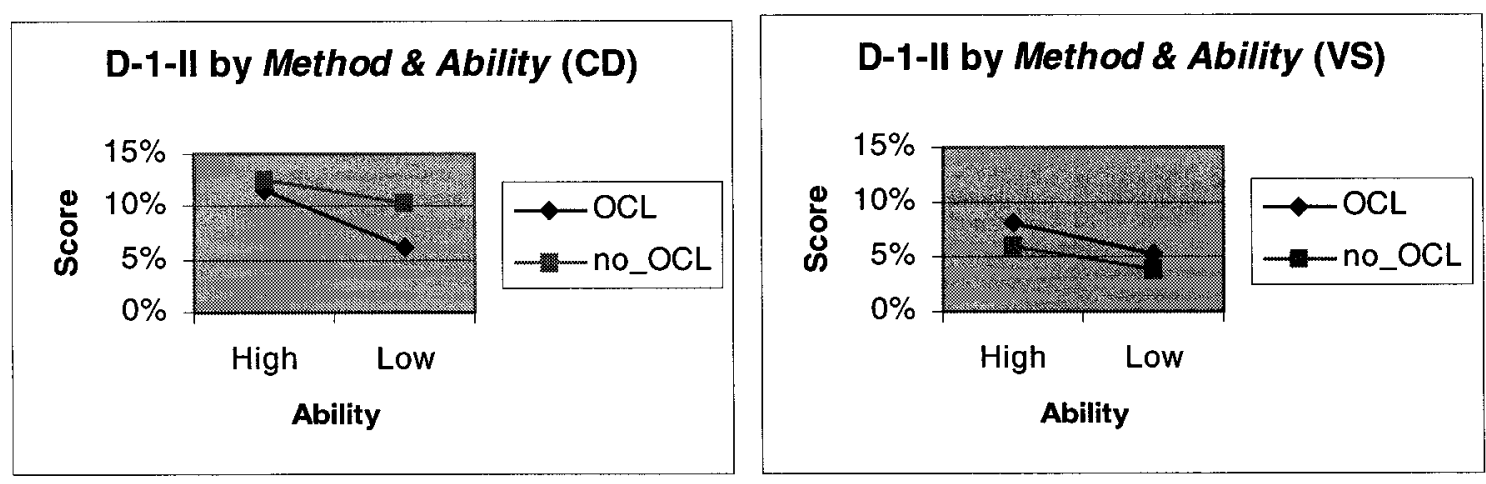
Figure 16. Graph of Means by Method and System - D-1-II
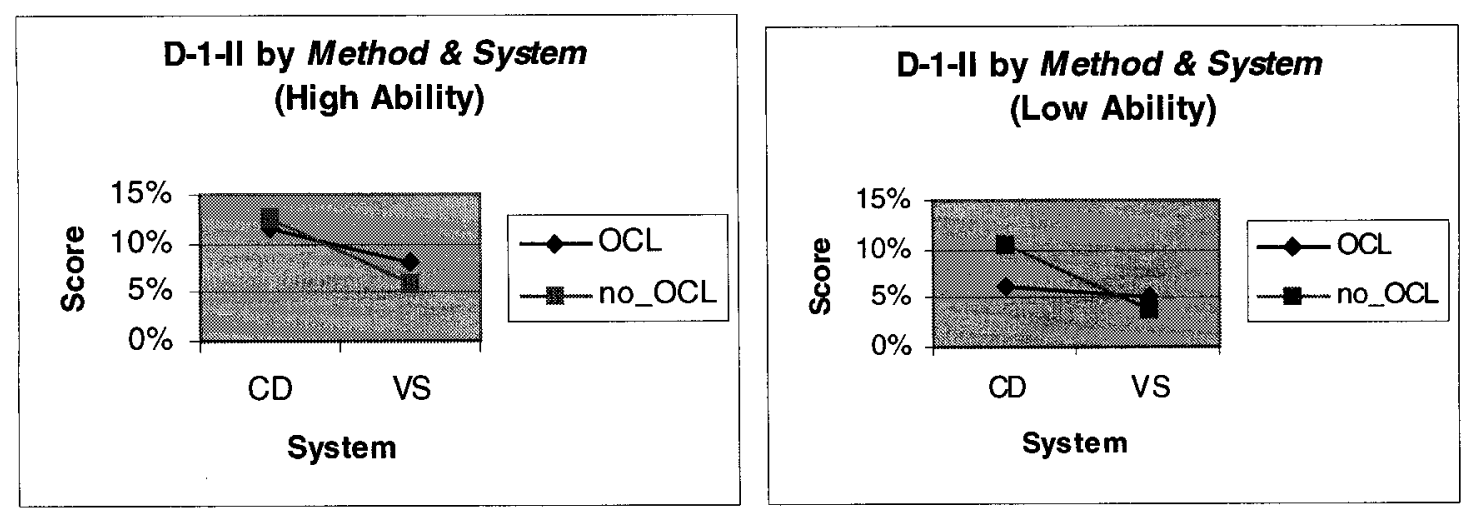

The survey results support the existence of significant effect of Ability. As shown in Table 87 on page 182, compared to the low ability subjects, the high ability subjects have less problems to find defects ( $\mathrm{p}$-value $=0.021$, mean (high ability) $=2.94$, mean (low ability) $=3.4$ ); and they know better what to look for in finding defects ( $p$-value $=0.003$, mean $($ high ability $)=3.14$, mean (low ability) $=2.42$ ). Moreover, the high ability subjects spend less time reading OCL expressions than the low ability subjects (p-value $=0.033$, mean(high ability) $=1.59$, mean (low ability $=2.09$ ). This suggests that the low ability subjects may have more difficulties to understand OCL compared to the high ability subjects.

The results of the survey questionnaire (Table 89) also support the main effect of System being significant: the subjects feel less time pressure when performing the task on the Cab Distribution system than on the Video Store system (p-value $=0.011$, mean $(C D)=$ 2.95$, mean $(\mathrm{VS})=3.55)$. 


\subsubsection{Defect Detection-3-II}

Testing the hypothesis - D-3-II

$\mathrm{H}_{0}: \mathrm{D}(\mathrm{OCL})=\mathrm{D}$ (no_OCL)

$\mathrm{H}_{\mathrm{a}}: \mathrm{D}(\mathrm{OCL})>\mathrm{D}$ (no_OCL)

The $t$-test yields a p-value of $0.003(\operatorname{mean}(\mathrm{OCL})=11.87 \%$, mean $($ no_OCL $)=7 \%)$. Since the null hypothesis is rejected at $\alpha=0.05$, using OCL has a significant positive effect on the dependent variable Defect Detection in this laboratory.

\section{Three-way ANOVA (Method, Ability \& System) - D-3-II}

Table 31 shows that Method has a significant main effect ( $\mathrm{p}$-value=0.002), explaining $11 \%$ of the total variation $\left(\mathrm{E}^{2}=0.11\right)$, which is particularly high. Ability also has a significant main effect $(p$-value $=0.024)$, explaining $5 \%$ of the total variation $\left(E^{2}=0.05\right)$.

Table 32 and Figure 18 show that using OCL has a positive effect for the high ability subjects, but not necessarily for the low ability subjects. This interaction between Method and Ability is significant ( $\mathrm{p}$-value $=0.038$ ), explaining $4 \%$ of the total variation $\left(\mathrm{E}^{2}=\right.$ 0.04).

Table 32 and Figure 17 show that using OCL has a positive effect for the Video Store system, but not necessarily for the Cab Distribution system. This interaction between Method and System is significant (p-value $=0.007$ ), explaining $8 \%$ of the total variation $\left(E^{2}=0.08\right)$.

Although Method has a significant main effect in this laboratory, there is no significant difference between the OCL subjects and the no_OCL subjects in the survey responses 
(Table 89 on page 183). All subjects are comfortable with the task. There are some differences between the high and the low ability subjects, as shown in Table 91 on page 183: compared to the low ability subjects, the high ability subjects are more confident in looking for defects ( $\mathrm{p}$-value $=0.033$, mean(high ability) $=3.24$, mean(low ability) $=$ 2.77); they also consider OCL to be more helpful (p-value(t-test) $=0.067$, pvalue $($ Wilcoxon test $)=0.047$, mean $($ high $)=2.56$, mean $($ low $)=3.05)$, as shown in Table 93 on page 184 . This supports the positive effect observed in the high ability subjects.

Table 31. Three-way ANOVA (Method, Ability \& System) - D-3-II

\begin{tabular}{|c|c|c|c|c|c|c|}
\hline Source & DF & Sum of Squares & Mean Square & F Ratio & Prob $>$ F & $E^{2}$ \\
\hline System & 1 & 0.01567961 & 0.01567961 & 3.6065 & 0.062 & 0.04 \\
\hline Ability & 1 & 0.02309928 & 0.02309928 & 5.3132 & $\mathbf{0 . 0 2 4}$ & 0.05 \\
\hline Method & 1 & 0.04698447 & 0.04698447 & 10.8071 & $\mathbf{0 . 0 0 2}$ & 0.11 \\
\hline Ability*System & 1 & 0.00498457 & 0.00498457 & 1.1465 & 0.288 & 0.01 \\
\hline Method*System & 1 & 0.03404013 & 0.03404013 & 7.8297 & $\mathbf{0 . 0 0 7}$ & 0.08 \\
\hline Ability*Method & 1 & 0.01949667 & 0.01949667 & 4.4845 & $\mathbf{0 . 0 3 8}$ & 0.04 \\
\hline Ability*Method *System & 1 & 0.00051305 & 0.00051305 & 0.118 & 0.732 & 0.001 \\
\hline Residual & 67 & 0.29128606 & 0.004348 & & & 0.66 \\
\hline Total & 74 & 0.44005072 & & & & \\
\hline
\end{tabular}

Table 32 Description statistics - D-3-II

\begin{tabular}{|l|l|c|c|c|}
\hline Method & Ability & System & Mean & Size \\
\hline OCL & High & CD & $14.15 \%$ & 8 \\
\cline { 2 - 5 } Mean: 11.87\%, Size: 36 & Mean: 15.55\%, Size: 17 & VS & $16.67 \%$ & 9 \\
\cline { 2 - 5 } & Low & CD & $8.49 \%$ & 11 \\
\cline { 2 - 5 } & Mean: 8.6\%, Size: 19 & VS & $8.75 \%$ & 8 \\
\hline \multirow{2}{*}{$\begin{array}{l}\text { no_OCL } \\
\text { Mean 7\%, Size: 39 }\end{array}$} & High & CD & $9.63 \%$ & 9 \\
\cline { 2 - 5 } & Mean: 7.27\%, Size: 17 & VS & $4.58 \%$ & 8 \\
\cline { 2 - 5 } & Low & CD & $11.52 \%$ & 11 \\
\cline { 2 - 5 } & Mean: 6.83\%, Size: 22 & VS & $2.12 \%$ & 11 \\
\hline
\end{tabular}


Figure 17. Graph of Means by Method and Ability - D-3-II
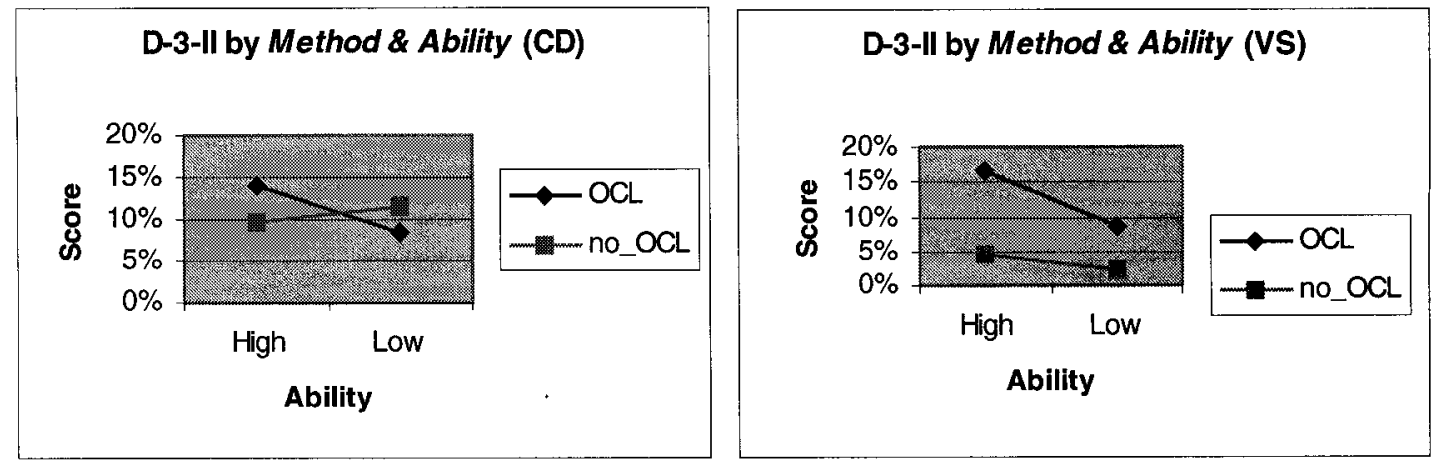

Figure 18. Graph of Means by Method and System - D-3-II
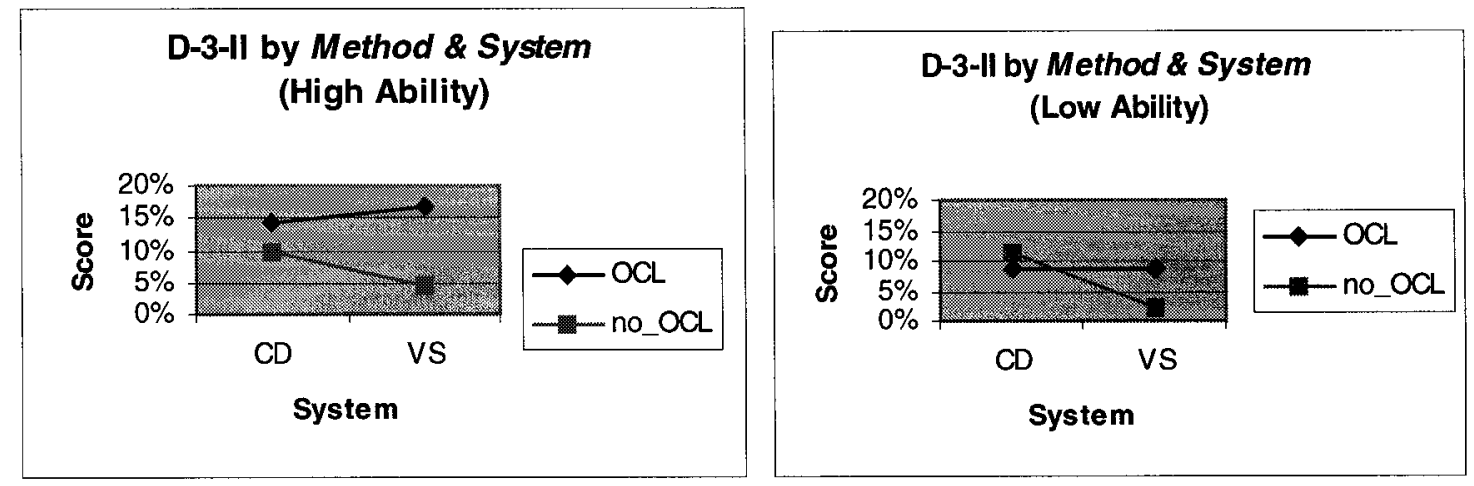

\subsubsection{D-1-II and D-3-II analysis results discussion}

\section{Main effects}

Method does not have a significant main effect in Laboratory 1, but has a significant positive effect in Laboratory 3: an effect of Order of Method exists. It is possible that the subjects are not prepared to use OCL effectively the first time they perform the task. After gaining more knowledge over time, the subjects start to take the advantage of using OCL. However, using OCL has different effects on the high and the low ability subjects (further discussed below). Ability has a significant main effect in both Laboratory 1 and Laboratory 3. System has a significant effect in Laboratory 1, but not in Laboratory 3. 


\section{Interaction effects}

There is no significant interaction effect in Laboratory 1. In Laboratory 3, both interaction effects in Method \& Ability and Method \& System are significant.

Using OCL has a positive effect on the high ability subjects, but not on the low ability subjects: only the performance of the $\mathrm{N} \_\mathrm{O}$ high ability subjects improves significantly from Laboratory 1 to Laboratory 3. This is also supported by the survey data: Table 96 on page 185 further shows that only the high ability subjects consider that OCL has helped more in Laboratory 3 than in Laboratory 1 (p-value $=0.029$, mean (high) $=3.31$, mean $($ low $)=2.56)$, such a difference is not present for the low ability subjects.

Using OCL also has a positive effect on the Video Store system, but not on the Cab Distribution system. OCL may have added complexity to a simpler system, but made the task easier in a more complex system.

Recall that Method has a significant positive effect for Laboratory 3 only. The significant interactions between Method and the other two factors are observed only for Laboratory 3 as well. Whether the interactions will always be visible whenever there is an effect of Method requires further investigation in future experiment, as it is possible that the interactions may not exist while the main effect of Method remains after the subjects gain more experience. 


\subsubsection{Experiment I and Experiment II}

This section compares the results of Experiment I and Experiment II. Section 4.1.3.1 describes the analysis results of all the observations (both attempts). Sections 4.1.3.2 and 4.1.3.3 provide a comparison of the two experiment trials for significant main and interaction effects for each laboratory (each attempt), respectively.

\subsubsection{All observations}

Table 33 summarizes the hypothesis testing results of all the observations for both experiment trials. Statistically, there is no difference in using and not using OCL in Experiment I (p-value $=0.302$ ), while using OCL has a significant positive effect in Experiment II (p-value $=0.05$ ). This result may be explained by the fact that the subjects of Experiment II have received more prior training in using OCL and in performing the tasks at hand than those of Experiment I: In Experiment I, the subjects are not yet prepared to apply OCL in detecting defects in UML system analysis documents; while in Experiment II, the subjects may be better prepared because of the extra OCL knowledge they have gained. Unfortunately, the survey results do not provide any supporting evidence. 
Table 33. All observation analysis of both experiment trials

\begin{tabular}{|l|l|c|}
\hline Experiment trial & Hypothesis & p-value \\
\hline \multirow{5}{*}{ Experiment I } & $\begin{array}{l}\mathrm{H}_{0}: \mathrm{D}(\mathrm{OCL})=\mathrm{D}(\text { no_OCL }) \\
\mathrm{H}_{\mathrm{a}}: \mathrm{D}(\mathrm{OCL})>\mathrm{D}(\text { no_OCL) }\end{array}$ & 0.302 \\
\cline { 2 - 3 } & $\begin{array}{l}\mathrm{H}_{0}: \text { Diff(N_O) }=\text { Diff(O_N) } \\
\mathrm{H}_{\mathrm{a}}: \text { Diff(N_O) }>\text { Diff(O_N) }\end{array}$ & $\mathbf{0 . 0 0 2}$ \\
\hline \multirow{5}{*}{ Experiment II } & $\begin{array}{l}\mathrm{H}_{0}: \mathrm{D}(\mathrm{OCL})=\mathrm{D}(\text { no_OCL) } \\
\mathrm{H}_{\mathrm{a}}: \mathrm{D}(\mathrm{OCL})>\mathrm{D}(\text { no_OCL })\end{array}$ & $\mathbf{0 . 0 5}$ \\
\cline { 2 - 3 } & $\begin{array}{l}\mathrm{H}_{0}: \text { Diff(N_O) }=\text { Diff(O_N) } \\
\mathrm{H}_{\mathrm{a}}: \text { Diff(N_O) }>\text { Diff(O_N) }\end{array}$ & $\mathbf{0 . 0 5}$ \\
\hline
\end{tabular}

In both experiment trials, the Order of Method has a significant effect: the difference between the performance of the subjects, when they use OCL and not use OCL, is larger for the N_O subjects than for the O_N subjects (p-value(Experiment $\mathrm{I}$ ) $=0.002, \mathrm{p}$ value $($ Experiment II $)=0.05)$, as shown in Table 34 .

In Experiment I, however, there is little evidence that using OCL has an effect overall, as there is a similar performance improvement from Laboratory 1 to Laboratory 3 for the O_N subjects and for the N_O subjects, which is probably due to the learning effect. The results of the second step of the analysis further show that using OCL does not have any significant main effect in either Laboratory 1 or Laboratory 3 in this experiment trial (discussed below).

In Experiment II, Order of Method has a confounding effect with Method. The N_O subjects' performance improvement from Laboratory 1 to Laboratory 3 is likely caused by the use of OCL. This indicates that using OCL may have helped the subjects in Laboratory 3 after more training is received. The results of the second step of data analysis confirm the above interpretation as discussed below. 
Table 34. Difference of OCL and no_OCL

\begin{tabular}{|c|c|c|c|c|c|c|}
\hline & & & \multicolumn{2}{|c|}{ Experiment I } & \multicolumn{2}{|c|}{ Experiment II } \\
\cline { 1 - 1 } & Lab 1 & Lab 3 & $\begin{array}{c}\text { Diff } \\
\text { (OCL - no_OCL) }\end{array}$ & p-value & $\begin{array}{c}\text { Diff } \\
\text { (OCL - no_OCL) }\end{array}$ & p-value \\
\hline O_N & OCL & No_OCL & $-6.36 \%$ & \multirow{2}{*}{$\mathbf{0 . 0 0 2}$} & $0.09 \%$ & \multirow{2}{*}{ 0.05 } \\
\cline { 1 - 3 } N_O & No_OCL & OCL & $7.95 \%$ & & $3.53 \%$ & \\
\hline
\end{tabular}

\subsubsection{Main effects}

The significant main effects of Experiment I and Experiment II are summarized in Table 35. Ability has significant main effects in both laboratories of both experiment trials for the Defect Detection dependent variable, which indicates that the individual's ability is key to such a task.

The factors which have significant main effects in D-3-I and D-1-II are the same: Ability and System. Recall that in Experiment II, the subjects have more time to mature their OCL knowledge compared to the subjects of Experiment I: they are trained more thoroughly in using OCL, and they have more time to receive further training between laboratories. Thus, it is possible that the subjects of Experiment II have a similar knowledge level of OCL in Laboratory 1 as that of the subjects of Experiment $\mathrm{I}$ in Laboratory 3.

Method has a significant main effect only for D-3-II, explaining $11 \%$ of the total variation, which is particularly high in practice. However, it does have significant interactions with Ability in both D-3-I and D-1-II (further discussed below). If the subjects' OCL knowledge level is considered similar for D-3-I as for D-1-II, a trend 
seems to exist: with more OCL knowledge gained, Method is likely to have a significant effect on the subjects' performance. This needs to be confirmed in future experiments.

System has significant main effects for D-3-I and D-1-II, but not for D-1-I or D-3-II. For D-3-II, there only exists a significant interaction between System and Method. It is possible that when the UML knowledge is lacking (in D-1-I), the complexity level of a system does not matter: the subjects are likely to perform poorly on any system. Once some UML knowledge is gained (in D-3-I), the gained knowledge impacts a simpler system (CD) more than a more complex system (VS). These are observed in Experiment I. In Experiment II, since the subjects received more prior training, the knowledge impacts a simpler system more than a more complex system right away during the first attempt at the task (in D-1-II). With further experience gained, System, in of itself, becomes less important (in D-3-II). Unfortunately, the only evidence provided by the survey results (Question 6 of Table 85 on page 182 and Table 95 on page 184) is that the low ability subjects consider the system to be less complex in D-3-II than D-1-II (p-value $=0.011, \operatorname{mean}(\mathrm{lab} 1)=2.32$, mean $(\mathrm{lab} 3)=2.8)$. More investigation is required to understand the trend in future experiments. 
Table 35. Comparing Experiment I and Experiment II - Main effects

\begin{tabular}{|c|c|c|c|c|c|c|}
\hline & \multicolumn{3}{|c|}{$\begin{array}{c}\text { Experiment I } \\
\text { (Two-way ANOVA) }\end{array}$} & \multicolumn{3}{c|}{$\begin{array}{c}\text { Experiment II } \\
\text { (Three-way ANOVA) }\end{array}$} \\
\hline & Effect & p-value & $\mathrm{E}^{2}$ & Effect & p-value & $\mathrm{E}^{2}$ \\
\hline Laboratory 1 & $\begin{array}{c}\text { Ability } \\
\text { (ANOVA with Method) }\end{array}$ & 0.038 & 0.15 & Ability & 0.03 & 0.06 \\
\cline { 5 - 8 } Laboratory 3 3 & $\begin{array}{c}\text { Ability } \\
\text { (ANOVA with Method) }\end{array}$ & 0.021 & 0.2 & Ability & 0.024 & 0.05 \\
\cline { 2 - 5 } & $\begin{array}{c}\text { System } \\
\text { (ANOVA with Method) }\end{array}$ & 0.008 & 0.31 & Method & 0.002 & 0.11 \\
\hline
\end{tabular}

\subsubsection{Interaction effects}

The significant interaction effects in Experiment $\mathrm{I}$ and Experiment $\mathrm{II}$ are summarized in Table 36. A significant interaction between Method and Ability is visible for Laboratory 3 of both experiment trials, and a significant interaction between Method and System is visible in Laboratory 3 of Experiment II only.

Table 36. Comparing Experiment I and Experiment II - Interaction effects

\begin{tabular}{|c|c|c|c|c|c|c|}
\hline & $\begin{array}{r}\text { ExI } \\
\text { (Two-v }\end{array}$ & $\begin{array}{l}\text { eriment } \\
\text { ay ANO }\end{array}$ & & $\begin{array}{r}\text { Expe } \\
\text { (Three-w }\end{array}$ & $\begin{array}{l}\text { iment II } \\
\text { ay ANO }\end{array}$ & \\
\hline & Effect & p-value & $E^{2}$ & Effect & p-value & $E^{2}$ \\
\hline \multirow{2}{*}{ Laboratory 3} & \multirow{2}{*}{$\begin{array}{l}\text { Interaction } \\
\text { of Method } \\
\text { and Ability }\end{array}$} & \multirow{2}{*}{0.024} & \multirow{2}{*}{0.19} & $\begin{array}{c}\text { Interaction of } \\
\text { Method and } \\
\text { Ability }\end{array}$ & 0.038 & 0.04 \\
\hline & & & & $\begin{array}{l}\text { Interaction of } \\
\text { Method and } \\
\text { System }\end{array}$ & 0.007 & 0.08 \\
\hline
\end{tabular}

There is a difference between Experiment I and Experiment II for the interaction between

Method and Ability in Laboratory 3. Recall that in Experiment I, using OCL seems to hinder the high ability subjects but help the low ability subjects; whereas in Experiment 
II, OCL helps the high ability subjects, but not the low ability subjects. Another difference is observed in the survey results: the survey results contradict laboratory observations from Experiment I, but support the laboratory observations from Experiment II.

The extra prior training and knowledge maturing time given to the subjects of Experiment II may explain the abovementioned difference. At first, the high ability subjects may have used their intuition to find the defects, without the help of OCL; whereas the low ability subjects may have used OCL in a mechanical way to detect document inconsistencies. When the subjects are much more comfortable with the tasks, UML system analysis, and OCL, the high ability subjects may have utilized OCL better than the low ability subjects. This needs further investigation in future experiments.

The significant interaction between Method and System is not present in any other laboratory, but D-3-II. Recall that there are indications from the survey data of D-3-I that there may exit interaction between Method \& System, although not visible in D-3-I, nor is Method. The interaction is only visible in D-3-II, and so is Method. It is possible that the significant interaction between Method \& System is visible whenever Method has a significant main effect. The fact, that using OCL has a positive effect only for the Video Store system, implies that OCL may have larger impact on a more complex system. These are left for future experiments to investigate further. 


\subsection{Comprehension (C)}

\subsubsection{Experiment I}

\subsubsection{Analyzing data of both laboratories}

All observations analysis

$\mathrm{H}_{0}: \mathrm{C}(\mathrm{OCL})=\mathrm{C}($ no_OCL $)$

$\mathrm{H}_{\mathrm{a}}: \mathrm{C}(\mathrm{OCL})>\mathrm{C}($ no_OCL)

A one-tailed paired t-test is used to test the above hypothesis. Overall, the subjects perform significantly better when OCL is used than when OCL is not used for the Comprehension task in Experiment I (p-value $=0.028$, mean $(\mathrm{OCL})=49.29 \%$, mean (no_OCL) $=42.86 \%$ ). Since the null hypothesis is rejected at $\alpha=0.05$, using OCL has a significant positive effect on the dependent variable Comprehension in this experiment trial.

\section{Testing effect of Order of Method}

$\mathrm{H}_{0}: \operatorname{Diff}\left(\mathrm{N} \_\mathrm{O}\right)=\operatorname{Diff}\left(\mathrm{O} \_\mathrm{N}\right)$

$\mathrm{H}_{\mathrm{a}}$ : Diff(N_O) $>$ Diff(O_N)

A one-tailed t-test is performed to test the above hypothesis. As shown in Table 37, the N_O subjects perform better when they apply OCL compared to when they do not $($ mean $($ Diff(N_O) $)=11.43 \%)$, while the O_N subjects perform only slightly better $\left(\right.$ mean $\left(\right.$ Diff $\left.\left.\left(\mathrm{O} \_\mathrm{N}\right)\right)=1.43 \%\right)$. Although the difference between the performance of using 
and not using OCL for the N_O subjects is much larger than for the O_N subjects, it is not statistically significant $(\mathrm{p}$-value $=0.061)$. (The $\mathrm{p}$-value is very close to 0.05 . A significant result may be observed if the sample size is larger.) The performance of the O_N subjects deteriorates by $1.43 \%$ from Laboratory 2 to Laboratory 4 . This indicates that the learning effect, if present, does not overcome the possible positive effect of using OCL. The absence of OCL may have hindered the subjects' performance in Laboratory 4. On the other hand, the N_O subjects' performance improves from Laboratory 2 to Laboratory 4 substantially. Thus, using OCL is likely to have a positive effect, at least during the second attempt at the task. The second step of the analysis is necessary to further investigate the effect of using OCL for each laboratory.

Table 37 Order of Methods applied

\begin{tabular}{|c|c|c|c|}
\hline & Lab 2 & Lab 4 & Diff(OCL - no_OCL) \\
\hline O_N & OCL & No_OCL & $1.43 \%$ \\
\hline N_O & No_OCL & OCL & $11.43 \%$ \\
\hline
\end{tabular}

\section{Comparing the performance between Laboratory 2 and Laboratory 4}

There is no significant difference between the subjects' overall performance in Laboratory 2 and in Laboratory 4 (p-value $=0.072$, mean $(1 \mathrm{lab} 2)=43.9 \%$, mean $(\mathrm{lab} 4)=$ $48.55 \%)$. Looking further into the laboratory observations, the N_O high ability subjects' performance improves significantly from Laboratory 2 to Laboratory 4 ( $\mathrm{p}$-value $=0.003$ ), as shown in Table 38. This suggests that the high ability subjects' performance improves faster than the low ability subjects when OCL is used during the second attempt at the task. 
Table 38. Comparing the same subjects (paired t-test)

\begin{tabular}{|c|c|c|c|c|}
\hline & Ability & Mean (Lab 2) & Mean (Lab 4) & p-value \\
\hline \multirow{2}{*}{ O_N } & High & $46.45 \%$ & $44.30 \%$ & 0.630 \\
\cline { 2 - 5 } & Low & $35.70 \%$ & $35.00 \%$ & 0.533 \\
\hline \multirow{2}{*}{ N_O } & High & $46.45 \%$ & $65.00 \%$ & $\mathbf{0 . 0 0 3}$ \\
\cline { 2 - 5 } & Low & $45.70 \%$ & $50.00 \%$ & 0.214 \\
\hline
\end{tabular}

The survey results (Table 101 on page 188, Table 103 on page 188, Table 105 on page 188, and Table 107 on page 189) support the above interpretation. There is not much difference between the two laboratories for the Comprehension task, except that the high ability subjects consider the questions much easier to answer in Laboratory 4 than in Laboratory $2(p$-value $=0.017$, mean $(\mathrm{lab} 2)=2.5$, mean $(\mathrm{lab} 4)=3.5)$. This indicates a learning effect for the high ability subjects.

\subsubsection{Comprehension-2-I}

\section{Testing the hypothesis -C-2-I}

$\mathrm{H}_{0}: \mathrm{C}(\mathrm{OCL})=\mathrm{C}($ no_OCL $)$

$\mathrm{H}_{\mathrm{a}}: \mathrm{C}(\mathrm{OCL})>\mathrm{C}($ no_OCL)

The $t$-test yields a p-value of $0.85($ mean $(\mathrm{OCL})=41.7 \%$, mean $($ no_OCL) $=45.9 \%)$.

Since the null hypothesis cannot be rejected at $\alpha=0.05$, there is no difference between using and not using OCL in this laboratory. Although the OCL subjects perform worse than the no_OCL subjects, there is no evidence that using OCL has a negative effect as well (corresponding p-value $=0.15$ ) 
Two-way ANOVA (Method \& Ability) - C-2-I

Table 39 shows that Ability does not have a significant effect ( $\mathrm{p}$-value $=0.082$ ) on Comprehension for Laboratory 2, nor does Method ( $\mathrm{p}$-value $=0.256$ ). Even though Figure 20 and Table 40 show a slight interaction between Method and Ability, it is not significant $(\mathrm{p}$-value $=0.293)$.

Table 39. Two-way ANOVA (Method and Ability) - C-2-I

\begin{tabular}{|c|c|c|c|c|c|c|}
\hline Source & DF & Sum of Squares & Mean Square & F Ratio & Prob > F & $E^{2}$ \\
\hline Ability & 1 & 0.03893545 & 0.03893545 & 3.2585 & 0.082 & 0.1 \\
\hline Method & 1 & 0.0161008 & 0.0161008 & 1.3475 & 0.256 & 0.04 \\
\hline Ability*Method & 1 & 0.01371399 & 0.01371399 & 1.1477 & 0.293 & 0.03 \\
\hline Residual & 28 & 0.33457341 & 0.011949 & & & 0.84 \\
\hline Total & 31 & 0.39867187 & & & & \\
\hline
\end{tabular}

Table 40. Descriptive statistics - C-2-I

\begin{tabular}{|c|c|c|c|c|c|c|}
\cline { 2 - 7 } \multicolumn{1}{c|}{} & \multicolumn{9}{c|}{ Ability } & \multicolumn{2}{c|}{ Low } & \multicolumn{2}{c|}{ All } \\
\cline { 2 - 7 } \multicolumn{1}{c|}{ High } & Mean & Size & Mean & Size & Mean & Size \\
\hline Method & $46.9 \%$ & 8 & $35.7 \%$ & 7 & $41.7 \%$ & 15 \\
\hline OCL & $47.2 \%$ & 9 & $44.4 \%$ & 8 & $45.9 \%$ & 17 \\
\hline No_OCL & 47 & $40.4 \%$ & 15 & $43.9 \%$ & 32 \\
\hline All Methods & $47.1 \%$ & 17 & 43 &
\end{tabular}

Figure 20. Graph of means - C-2-I

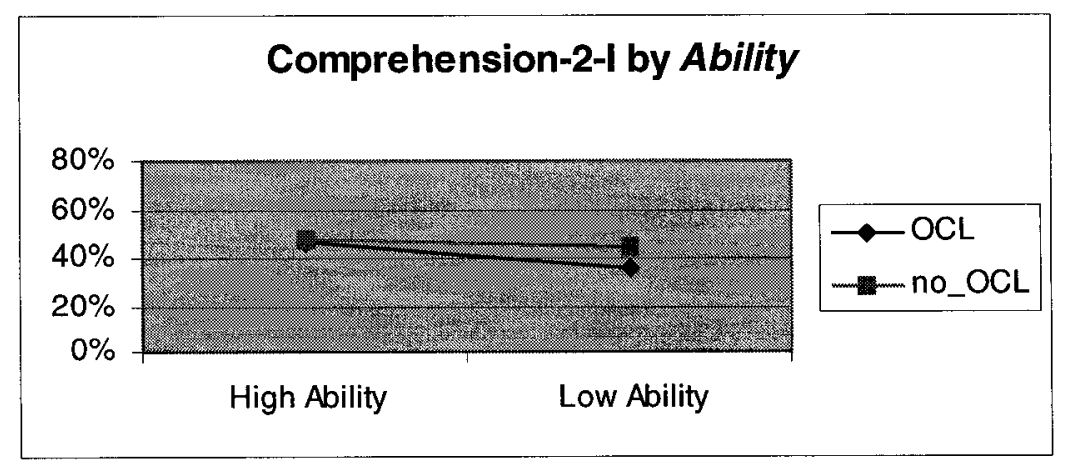


Two-way ANOVA (Method \& System) - C-2-I

Table 41 shows that neither Method nor System has significant effects on Comprehension for Laboratory 2 (p-value $=0.313$ and 0.271 , respectively). There is no significant interaction between Method and System either (p-value $=0.935)$, as shown in Figure 21 and Table 42.

Table 41. Two-way ANOVA (Method and System) - C-2-I

\begin{tabular}{|c|c|c|c|c|c|c|}
\hline Source & DF & Sum of Squares & Mean Square & F Ratio & Prob $>$ F & $E^{2}$ \\
\hline System & 1 & 0.01660113 & 0.01660113 & 1.2634 & 0.271 & 0.04 \\
\hline Method & 1 & 0.01384522 & 0.01384522 & 1.0537 & 0.313 & 0.03 \\
\hline System*Method & 1 & 0.0000903 & 0.0000903 & 0.0069 & 0.935 & 0.0002 \\
\hline Residual & 28 & 0.36790675 & 0.01314 & & & 0.92 \\
\hline Total & 31 & 0.39867187 & & & & \\
\hline
\end{tabular}

Table 42. Descriptive Statistics - C-2-I

\begin{tabular}{|c|c|c|c|c|c|c|}
\hline \multirow[b]{3}{*}{ Method } & \multicolumn{6}{|c|}{ System } \\
\hline & \multicolumn{2}{|c|}{$\mathrm{CD}$} & \multicolumn{2}{|c|}{ VS } & \multicolumn{2}{|c|}{ All } \\
\hline & Mean & Size & Mean & Size & Mean & Size \\
\hline OCL & $44.3 \%$ & 7 & $39.4 \%$ & 8 & $41.7 \%$ & 15 \\
\hline No_OCL & $48.1 \%$ & 8 & $43.9 \%$ & 9 & $45.9 \%$ & 17 \\
\hline All Methods & $46.4 \%$ & 15 & $41.8 \%$ & 17 & $43.9 \%$ & 32 \\
\hline
\end{tabular}

Figure 21. Graph of Means - C-2-I

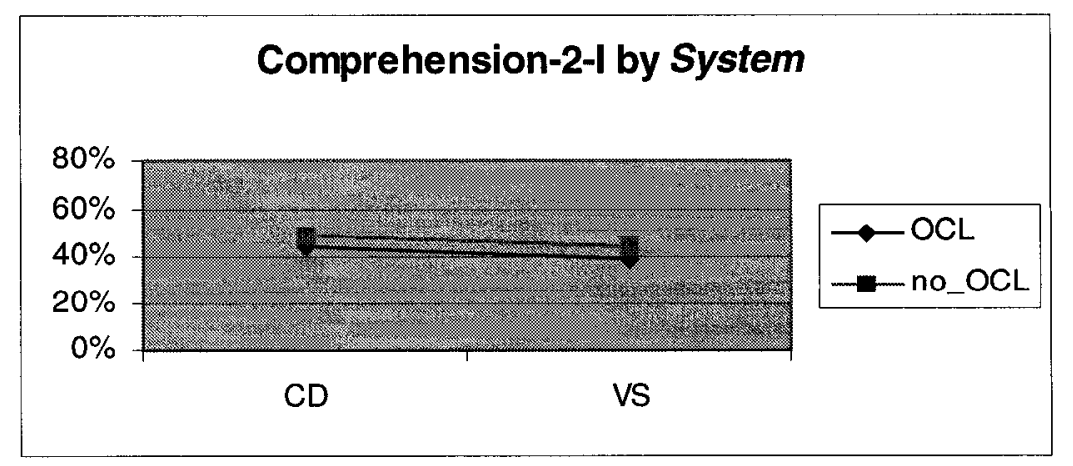




\section{Combining two ANOVA analyses - C-2-I}

Combining the results of both two-way ANOVA analyses, there are neither significant main effects, nor significant interaction effects for this laboratory.

\subsubsection{Comprehension-4-I}

Testing the hypothesis - C-4-I

$\mathrm{H}_{0}: \mathrm{C}(\mathrm{OCL})=\mathrm{C}($ no_OCL $)$

$\mathrm{H}_{\mathrm{a}}: \mathrm{C}(\mathrm{OCL})>\mathrm{C}($ no_OCL $)$

The $t$-test yields a p-value of $0.002($ mean $(\mathrm{OCL})=57.9 \%$, mean $($ no_OCL $)=39.65 \%)$.

Since the null hypothesis is rejected at $\alpha=0.05$, using OCL has a significantly positive effect on the Comprehension dependent variable in this laboratory.

\section{Two-way ANOVA (Method \& Ability) - C-4-I}

As shown in Table 43, both Method and Ability have significant main effects (p-values of 0.035 and 0.003 , respectively) on the subjects' capacity to answer questions about the systems' logic. As illustrated by Figure 22 and Table 44, using OCL has a positive effect on both high and low ability subjects; the high ability subjects perform better than the low ability subjects, irrespective of which method is used. Method explains $27 \%$ of the total variation $\left(E^{2}=0.27\right)$, which is particularly significant and even higher than the effect of Ability $\left(\mathrm{E}^{2}=0.12\right)$. Although only the N_O high ability subjects' performance improves significantly from Laboratory 2 to Laboratory 4 , there is no significant interaction between Method and Ability (p-value $=0.604$ ). 
Table 43. Two-way ANOVA (Method \& Ability) - C-4-I

\begin{tabular}{|c|c|c|c|c|c|c|}
\hline Source & DF & Sum of Squares & Mean Square & F Ratio & Prob $>$ F & $E^{2}$ \\
\hline Ability & 1 & 0.10321429 & 0.10321429 & 4.9828 & $\mathbf{0 . 0 3 5}$ & 0.12 \\
\hline Method & 1 & 0.22321429 & 0.22321429 & 10.7759 & $\mathbf{0 . 0 0 3}$ & 0.27 \\
\hline Ability*Method & 1 & 0.00571429 & 0.00571429 & 0.2759 & 0.604 & 0.007 \\
\hline Residual & 24 & 0.49714286 & 0.020714 & & & 0.6 \\
\hline Total & 27 & 0.82928571 & & & & \\
\hline
\end{tabular}

Table 44. Descriptive statistics - C-4-I

\begin{tabular}{|c|c|c|c|c|c|c|}
\cline { 2 - 7 } \multicolumn{1}{c|}{} & \multicolumn{9}{c|}{ Ability } & \multicolumn{2}{c|}{ Low } & \multicolumn{2}{c|}{ All } \\
\cline { 2 - 7 } \multicolumn{1}{c|}{ High } & Mean & Size & Mean & Size & Mean & Size \\
\hline Method & $65 \%$ & 7 & $50 \%$ & 7 & $57.5 \%$ & 14 \\
\hline OCL & $44.3 \%$ & 7 & $35 \%$ & 7 & $39.65 \%$ & 14 \\
\hline No_OCL & $54.7 \%$ & 14 & $42.5 \%$ & 14 & $48.55 \%$ & 28 \\
\hline All Methods & 54 & \multicolumn{4}{c}{} & \\
\hline
\end{tabular}

Figure 22. Graph of means - C-4-I

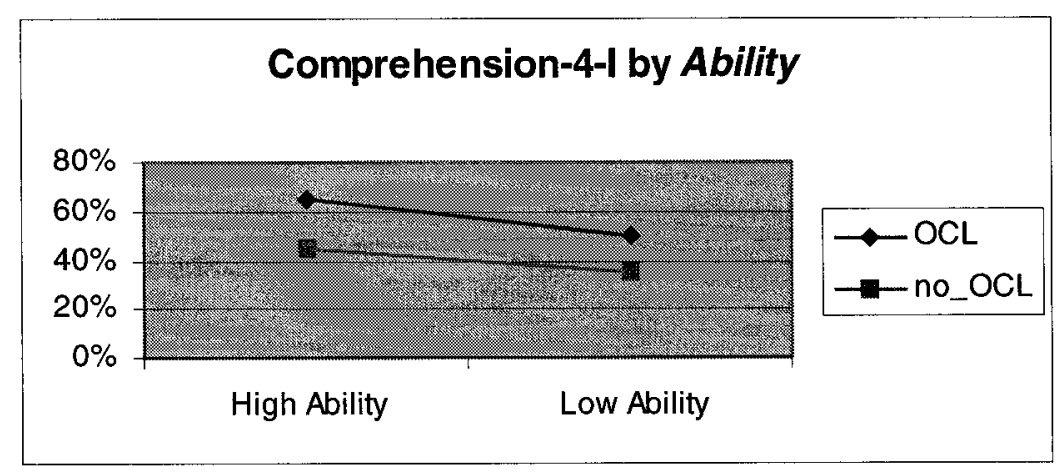

Two-way ANOVA (Method \& System) - C-4-I

Table 45 shows that both Method and System have significant main effects ( $\mathrm{p}$-value = 0.001 and 0.007 , respectively), explaining $28 \%\left(E^{2}=0.28\right)$ and $18 \%\left(E^{2}=0.18\right)$ of the total variation, respectively. Figure 23 and Table 46 show that using OCL has a positive effect on the subjects' performance for both systems; the subjects perform better for the Cab Distribution system than for the Video Store system, irrespective of the method used. 
Although using OCL seems to have a larger effect for the Video Store system, the interaction between Method and System is not significant (p-value=0.073).

Table 45. Two-way ANOVA (Method and System) - C-4-I

\begin{tabular}{|c|c|c|c|c|c|c|}
\hline Source & DF & Sum of Squares & Mean Square & F Ratio & Prob > F & $\mathrm{E}^{2}$ \\
\hline System & 1 & 0.14546288 & 0.14546288 & 8.8091 & $\mathbf{0 . 0 0 7}$ & 0.18 \\
\hline Method & 1 & 0.23467947 & 0.23467947 & 14.212 & $\mathbf{0 . 0 0 1}$ & 0.28 \\
\hline System*Method & 1 & 0.05815105 & 0.05815105 & 3.5216 & 0.073 & 0.07 \\
\hline Residual & 24 & 0.39630556 & 0.016513 & & & 0.48 \\
\hline Total & 27 & 0.82928571 & & & & \\
\hline
\end{tabular}

Table 46. Descriptive statistics - C-4-I

\begin{tabular}{|c|c|c|c|c|c|c|}
\hline \multirow[b]{3}{*}{ Method } & \multicolumn{6}{|c|}{ System } \\
\hline & \multicolumn{2}{|c|}{$\mathrm{CD}$} & \multicolumn{2}{|c|}{ VS } & \multicolumn{2}{|c|}{ All } \\
\hline & Mean & Size & Mean & Size & Mean & Size \\
\hline $\mathrm{OCL}$ & $59.45 \%$ & 9 & $54 \%$ & 5 & $57.5 \%$ & 14 \\
\hline No_OCL & $50 \%$ & 8 & $25.85 \%$ & 6 & $39.65 \%$ & 14 \\
\hline All Methods & $55 \%$ & 17 & $38.65 \%$ & 11 & $48.55 \%$ & 28 \\
\hline
\end{tabular}

Figure 23. Graph of Means - C-4-I

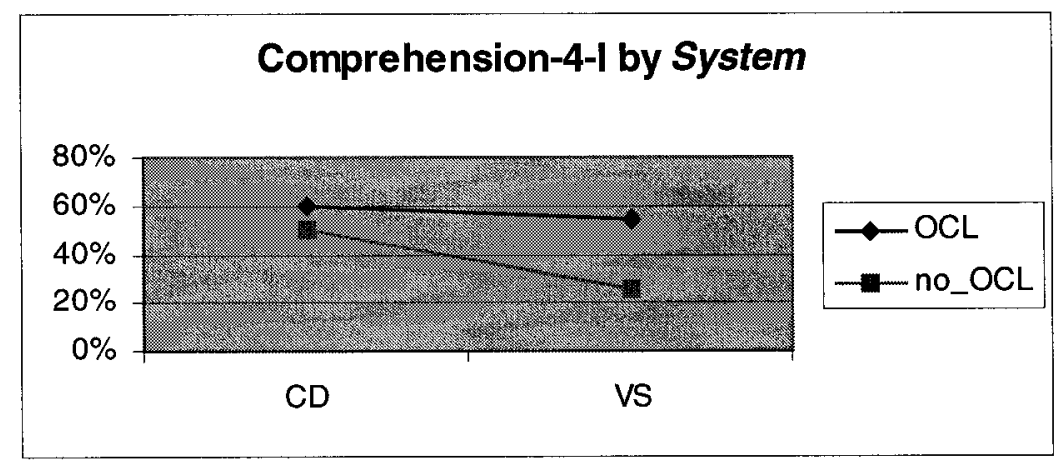

\section{Combining two ANOVA analyses- C-4-I}

Combining both two-way ANOVA results, the main effects of all the three factors, Method, System and Ability, are significant. There is no significant interaction among the factors. 
The results of laboratory 4 survey questionnaire (Table 98 on page 186) support Method having a significant main effect. Compared to the no_OCL subjects, the OCL subjects spend less time in understanding the system (p-value $=0.006$, mean $(\mathrm{OCL})=3.87$, mean $($ no_OCL $)=2.86$ ) and consider the Comprehension questions less difficult to answer $(\mathrm{p}$-value $=0.0002$, mean $(\mathrm{OCL})=3.5$, mean $($ non-OCL $)=1.92)$.

The survey results do not provide any support for Ability having a significant main effect. There is no difference between the low and the high ability subjects' responses, as shown in Table 100 on page 187.

Table 102 on page 187 shows that the subjects consider OCL easier to understand for the Cab Distribution system than for the Video Store system (p-value $=0.001$, mean $(C D)=$ 1.88 , mean (VS) $=3.33$, which confirms the significant main effect of System. However, recall that the perception is opposite in Laboratory 3 for the Defect Detection task (Section 4.1.1.2). Such difference is difficult to interpret, and it is possibly due to the difference between the tasks: the Defect Detection task is considered to be more complex than the Comprehension task, and using OCL may bring more benefits when a more complex task is performed on a more complex system. Furthermore, such difference between the abovementioned perceptions is not visible in Experiment II. The discrepancies can be investigated further through subject interview in future experiments. 


\subsubsection{C-2-I and C-4-I analysis results discussion}

\section{Main effects}

Although using OCL has a positive effect on Comprehension, considering all the observations from both attempts at the task, no significant main effect exists in C-2-I, while all the three factors have significant main effects in C-4-I. The OCL subjects perform slightly, but not significantly, worse than the no_OCL subjects overall in C-2-I, while significantly better in C-4-I. Order of Method does have an effect on the subjects' performance. There is no improvement in the $\mathrm{O} \_\mathrm{N}$ subjects' performance from Laboratory 2 to Laboratory 4 . The absence of OCL may have caused this performance deterioration. On the other hand, the N_O subjects' performance improves from Laboratory 2 to Laboratory 4 . The presence of OCL may, together with the learning effect, have helped the N_O subjects to perform better in Laboratory 4. These clearly demonstrate the positive effect of using OCL.

Both Ability and System have significant main effects only in Laboratory 4: the high ability subjects perform better than the low ability subjects; the subjects perform better for the Cab Distribution system than for the Video Store system. It is possible that the subjects are not familiar with the task in Laboratory 2 . As more experience is gained, the high ability subjects may have improved faster than the low ability subjects, resulting in better performance in Laboratory 4. Meanwhile, the gained experience may have larger positive effects on simpler systems. 


\section{Interaction effects}

There is no significant interaction effect for the Comprehension task when considering the observations from each attempt at the task in this experiment trial.

\subsubsection{Experiment II}

\subsubsection{Analyzing data of both Laboratory 2 and Laboratory 4}

All observations analysis

$\mathrm{H}_{0}: \mathrm{C}(\mathrm{OCL})=\mathrm{C}($ no_OCL $)$

$\mathrm{H}_{\mathrm{a}}: \mathrm{C}(\mathrm{OCL})>\mathrm{C}($ no_OCL)

The one-tailed paired t-test is used to test the above hypothesis. The subjects perform significantly better when OCL is used than when OCL is not used for the Comprehension task in Experiment $\Pi$ ( $\mathrm{p}$-value $=0.0001$, mean $(\mathrm{OCL})=49.87 \%$, mean (no_OCL) $=$ $42.36 \%$ ). Since the null hypothesis is rejected at $\alpha=0.05$, using OCL has a significant positive effect on the dependent variable Comprehension in this experiment trial.

\section{Testing order of Method of diff $\left(O C L-n o \_O C L\right)$}

$\mathrm{H}_{0}: \operatorname{Diff}\left(\mathrm{N} \_\mathrm{O}\right)=\operatorname{Diff}\left(\mathrm{O} \_\mathrm{N}\right)$

$\mathrm{H}_{\mathrm{a}}:$ Diff(N_O) $>$ Diff $\left(\mathrm{O} \_\mathrm{N}\right)$

The one-tailed t-test is used to test the above hypothesis. The N_O subjects perform better when they use OCL compared to when they do not $\left(\right.$ mean $\left.\left(\operatorname{Diff}\left(\mathrm{N} \_\mathrm{O}\right)\right)=10.79 \%\right)$. The O_N subjects also perform better when they use OCL compared to when they do not $\left(\right.$ mean $\left.\left(\operatorname{Diff}\left(\mathrm{O} \_\mathrm{N}\right)\right)=4.03 \%\right)$, as shown in Table 47 . The difference between them is 
significant ( $\mathrm{p}$-value $=0.04$ ). It is possible that the positive effect of using OCL is stronger for Laboratory 4 than for Laboratory 2.

Table 47 Order of Methods applied

\begin{tabular}{|c|c|c|c|}
\hline & Lab 2 & Lab 4 & Diff(OCL - no_OCL) \\
\hline O_N & OCL & No_OCL & $4.03 \%$ \\
\hline N_O & No_OCL & OCL & $10.79 \%$ \\
\hline
\end{tabular}

\section{Comparing performance between Laboratory 2 and Laboratory 4}

The subjects' performance is improved significantly overall from Laboratory 2 to Laboratory $4(\mathrm{p}$-value $=0.044$, mean $(\mathrm{lab} 2)=44 \%$, mean $(\mathrm{lab} 4)=47 \%)$. This is also supported by the survey results. Table 110 on page 191 and Table 112 on page 191 show that the subjects are more comfortable with the task: having less problems to understand the system $(p$-value $=0.02$, mean $(\operatorname{lab} 2)=2.68$, mean $(l a b 4)=2.43)$, requiring less time to complete the task (p-value $=0.002$, mean $(\operatorname{lab} 2)=2.99$, mean $($ lab4 $)=3.48)$ ), and spending more time on using OCL in Laboratory 4 than in Laboratory 2 (p-value $=0.039$, mean $(\mathrm{lab} 2)=1.62$, mean $(\mathrm{lab} 4)=2.04)$.

Further, Table 48 shows that only the N_O subjects' performance improves significantly from Laboratory 2 to Laboratory 4 (p-value(high ability) $=0.003$ p-value(low ability) $=$ 0.007). There is no such improvement for the O_N subjects. In fact, all the O_N subjects perform worse from Laboratory 2 to Laboratory 4 . This further demonstrates the positive effect of using OCL already observed. 
Table 48. Comparing the same subjects (paired t-test)

\begin{tabular}{|c|c|c|c|c|}
\hline & Ability & Mean (Lab 2) & Mean (Lab 4) & p-value \\
\hline \multirow{2}{*}{ O_N } & High & $50.56 \%$ & $44.06 \%$ & 0.963 \\
\cline { 2 - 5 } & Low & $44.30 \%$ & $42.25 \%$ & 0.68 \\
\hline \multirow{2}{*}{ N_O } & High & $42.94 \%$ & $56.77 \%$ & $\mathbf{0 . 0 0 3}$ \\
\cline { 2 - 5 } & Low & $40.71 \%$ & $49.05 \%$ & $\mathbf{0 . 0 0 7}$ \\
\hline
\end{tabular}

\subsubsection{Comprehension-2-II}

Testing the hypothesis- C-2-II

$\mathrm{H}_{0}: \mathrm{C}(\mathrm{OCL})=\mathrm{C}($ no_OCL $)$

$\mathrm{H}_{\mathrm{a}}: \mathrm{C}(\mathrm{OCL})>\mathrm{C}($ no_OCL $)$

The $t$-test yields a p-value of 0.017 (mean $(\mathrm{OCL})=46 \%$, mean $($ no_OCL $)=41 \%)$. Since the null hypothesis is rejected at $\alpha=0.05$, using OCL has a significantly positive effect on the Comprehension dependent variable in this laboratory.

\section{Three-way ANOVA (Method, Ability \& System) - C-2-II}

Table 49 shows that only Method has a significant main effect in this laboratory, explaining $7 \%$ of the total variation, while Ability and System do not. Using OCL has a positive effect on the subjects' performance, regardless of the subjects' ability or the system used, as shown in Table 50, Figure 24 and Figure 25. There is no significant interaction among the factors. 
Table 49. Three-way ANOVA (Method, Ability \& System)- C-2-II

\begin{tabular}{|c|c|c|c|c|c|c|}
\hline Source & DF & Sum of Squares & Mean Square & F Ratio & Prob > F & $\mathrm{E}^{2}$ \\
\hline System & 1 & 0.04166417 & 0.04166417 & 2.4801 & 0.112 & 0.03 \\
\hline Ability & 1 & 0.02268567 & 0.02268567 & 1.3504 & 0.249 & 0.02 \\
\hline Method & 1 & 0.09000712 & 0.09000712 & 5.3577 & $\mathbf{0 . 0 2 4}$ & 0.07 \\
\hline Ability*System & 1 & 0.0162834 & 0.0162834 & 0.9693 & 0.328 & 0.01 \\
\hline Method*System & 1 & 0.00070751 & 0.00070751 & 0.0421 & 0.838 & 0.0005 \\
\hline Ability*Method & 1 & 0.020805 & 0.020805 & 1.2384 & 0.27 & 0.02 \\
\hline Ability*Method *System & 1 & 0.01746169 & 0.01746169 & 1.0394 & 0.312 & 0.01 \\
\hline Residual & 69 & 1.1591761 & 0.0168 & & & 0.86 \\
\hline Total & 76 & 1.3536883 & & & & \\
\hline
\end{tabular}

Table 50. Descriptive statistics - C-2-II

\begin{tabular}{|c|c|c|c|c|}
\hline Method & Ability & System & Mean & Size \\
\hline \multirow{4}{*}{$\begin{array}{l}\text { OCL } \\
\text { Mean: } 47 \% \\
\text { Size: } 39\end{array}$} & \multirow{2}{*}{$\begin{array}{l}\text { High } \\
\text { Mean: } 51 \%, \text { Size: } 17\end{array}$} & $\mathrm{CD}$ & $49.4 \%$ & 9 \\
\hline & & VS & $53.6 \%$ & 8 \\
\hline & \multirow{2}{*}{$\begin{array}{l}\text { Low } \\
\text { Mean: } 44 \%, \text { Size } 22\end{array}$} & $\mathrm{CD}$ & $41.50 \%$ & 10 \\
\hline & & VS & $46.75 \%$ & 12 \\
\hline \multirow{4}{*}{$\begin{array}{l}\text { no_OCL } \\
\text { Mean: } 41 \% \\
\text { Size } 40\end{array}$} & \multirow{2}{*}{$\begin{array}{l}\text { High } \\
\text { Mean: } 41 \% \text {, Size: } 19\end{array}$} & $\mathrm{CD}$ & $41.67 \%$ & 9 \\
\hline & & VS & $41.00 \%$ & 10 \\
\hline & \multirow{2}{*}{$\begin{array}{l}\text { Low } \\
\text { Mean 35\%, Size } 21\end{array}$} & $\mathrm{CD}$ & $34.00 \%$ & 10 \\
\hline & & VS & $46.82 \%$ & 11 \\
\hline
\end{tabular}

Figure 24. Graph of Means by Method and Ability - C-2-II
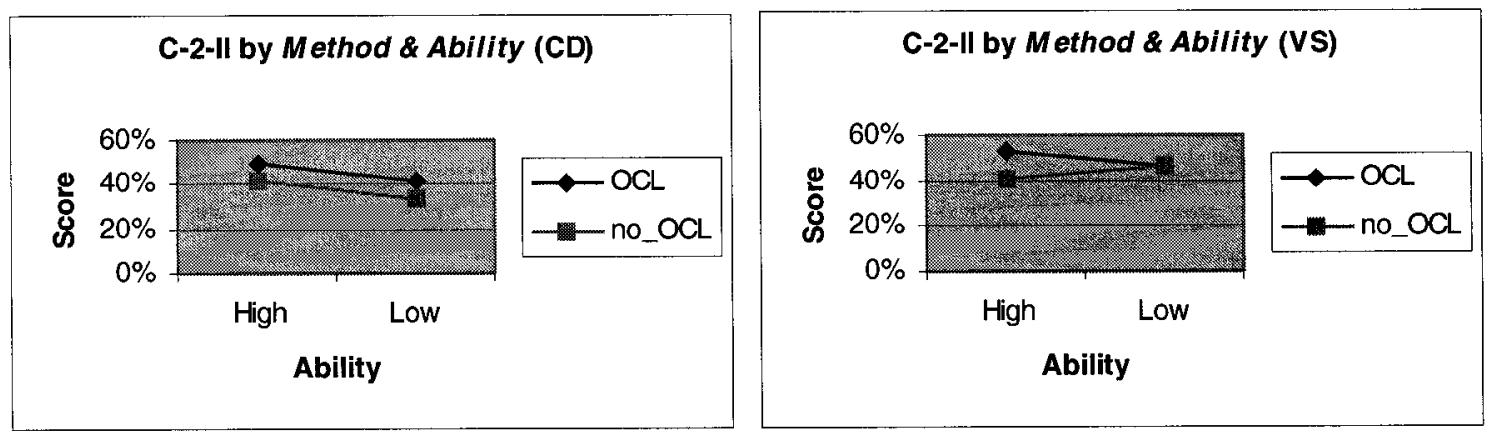
Figure 25. Graph of Means by Method and System - C-2-II
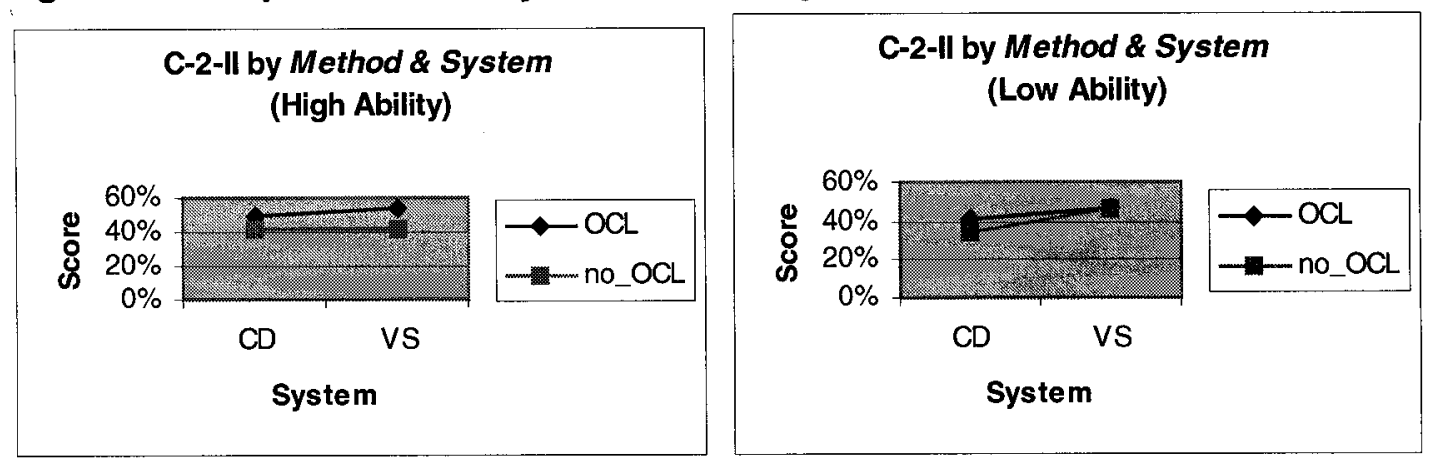

However, the survey results do not support Method having a significant effect. There is no significant difference between the OCL and the no_OCL subjects' responses to the survey questionnaire (Table 105 on page 189).

\subsubsection{Comprehension_4_II}

\section{Testing the hypothesis-C-4-II}

$\mathrm{H}_{0}: \mathrm{C}(\mathrm{OCL})=\mathrm{C}($ no_OCL $)$

$\mathrm{H}_{\mathrm{a}}: \mathrm{C}(\mathrm{OCL})>\mathrm{C}($ no_OCL $)$

The $t$-test yields a p-value of $0.013(\operatorname{mean}(\mathrm{OCL})=51 \%$, mean $($ no_OCL) $=44 \%)$. Since the null hypothesis is rejected at $\alpha=0.05$, using OCL has a significantly positive effect on the Comprehension dependent variable in this laboratory.

\section{Three-way ANOVA (Method, Ability \& System) - C-4-II}

Table 51, Table 52, Figure 26 and Figure 27 show that all the three factors, Method, System and Ability have significant main effects (p-value $=0.015,0.014$ and 0.018 , respectively): using OCL has positive effect on the subjects' performance overall; the high ability subjects perform better than the low ability subjects; and the subjects perform 
better for the Cab Distribution system than for the Video Store system. Each of them explains about $7 \%$ of the total variation $\left(E^{2}=0.07,0.07\right.$, and 0.06 , respectively). There is no significant interaction among them.

Table 51. Three-way ANOVA (Method, Ability \& System) - C-4-II

\begin{tabular}{|c|c|c|c|c|c|c|}
\hline Source & DF & Sum of Squares & Mean Square & F Ratio & Prob $>$ F & $E^{2}$ \\
\hline System & 1 & 0.10139793 & 0.10139793 & 6.3258 & $\mathbf{0 . 0 1 4}$ & 0.07 \\
\hline Ability & 1 & 0.09459393 & 0.09459393 & 5.9013 & $\mathbf{0 . 0 1 8}$ & 0.06 \\
\hline Method & 1 & 0.09998698 & 0.09998698 & 6.2378 & $\mathbf{0 . 0 1 5}$ & 0.07 \\
\hline Ability*System & 1 & 0.01610433 & 0.01610433 & 1.0047 & 0.32 & 0.01 \\
\hline Method*System & 1 & 0.00127611 & 0.00127611 & 0.0796 & 0.779 & 0.0009 \\
\hline Ability*Method & 1 & 0.02442935 & 0.02442935 & 1.524 & 0.221 & 0.02 \\
\hline Ability*Method *System & 1 & 0.02625548 & 0.02625548 & 1.638 & 0.205 & 0.02 \\
\hline Residual & 69 & 1.1060196 & 0.016029 & & & 0.76 \\
\hline Total & 76 & 1.4577273 & & & & \\
\hline
\end{tabular}

Table 52. Descriptive statistics - C-4-II

\begin{tabular}{|c|c|c|c|c|}
\hline Method & Ability & System & Mean & Size \\
\hline \multirow{4}{*}{$\begin{array}{l}\text { OCL } \\
\text { Mean: } 51 \% \text {, Size: } 40\end{array}$} & \multirow{2}{*}{$\begin{array}{l}\text { High } \\
\text { Mean: } 57 \% \text {, Size: } 17\end{array}$} & $\mathrm{CD}$ & $59.44 \%$ & 9 \\
\hline & & VS & $53.75 \%$ & 8 \\
\hline & \multirow{2}{*}{$\begin{array}{l}\text { Low } \\
\text { Mean: } 46 \% \text {, Size: } 23\end{array}$} & $C D$ & $49.58 \%$ & 12 \\
\hline & & VS & $42.27 \%$ & 11 \\
\hline \multirow{4}{*}{$\begin{array}{l}\text { no_OCL } \\
\text { Mean: } 44 \% \text {, Size: } 37\end{array}$} & \multirow{2}{*}{$\begin{array}{l}\text { High } \\
\text { Mean: 45\%, Size: } 17\end{array}$} & $\mathrm{CD}$ & $53.13 \%$ & 8 \\
\hline & & VS & $38.33 \%$ & 9 \\
\hline & \multirow{2}{*}{$\begin{array}{l}\text { Low } \\
\text { Mean: } 42 \% \text {, Size: } 20\end{array}$} & $\mathrm{CD}$ & $43.00 \%$ & 10 \\
\hline & & VS & $41.50 \%$ & 10 \\
\hline
\end{tabular}


Figure 26. Graph of Means by Method and Ability - C-4-II
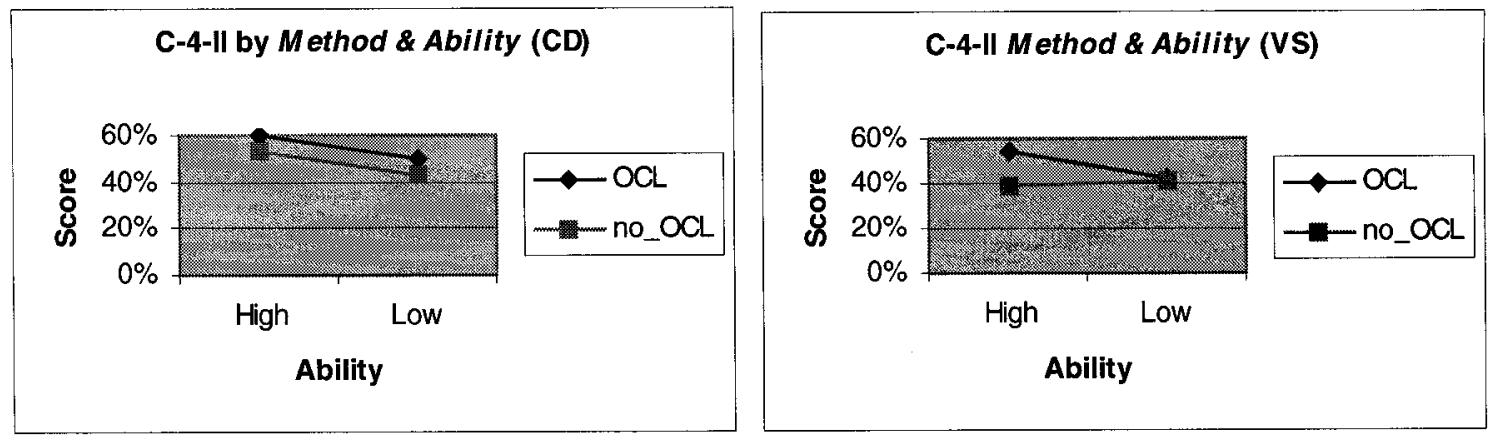

Figure 27. Graph of Means by Method and System - C-4-II
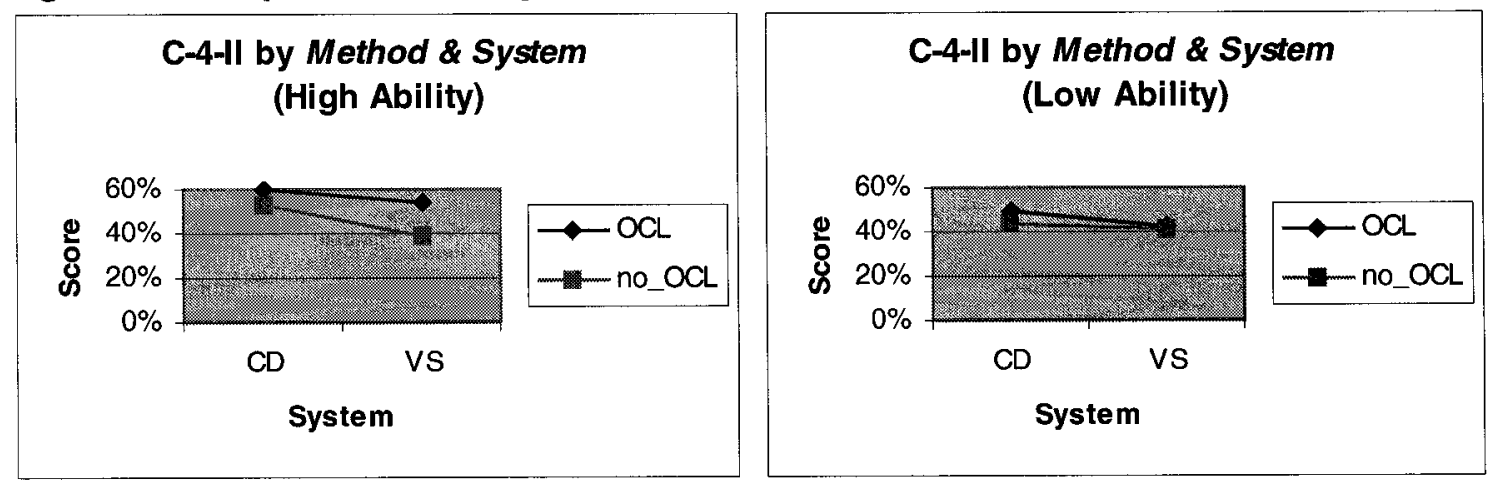

The survey results support all the three factors having significant main effects. Table 107 on page 190 shows that although the OCL subjects feel more time pressure (p-value $=$ 0.017 , mean $(\mathrm{OCL})=3.22$, mean $($ no_OCL $)=3.47)$, they consider the questions easier to answer compared to the no_OCL subjects $(\mathrm{p}$-value $=0.003$, mean $(\mathrm{OCL})=2.95$, mean $($ no_OCL $)=2.3$ ). This supports Method having a significant effect.

Table 109 on page 190 shows that there is little significant difference between the high and the low ability subjects, except that the high ability subjects spend less time reading and using OCL than the low ability subjects $(\mathrm{p}$-value $=0.018$, mean(high) $=1.56$, 
mean $($ low $)=2.2$. It is possible that the low ability subjects may not be as skilled in using OCL as the high ability subjects. This supports Ability having a significant effect.

Table 111 on page 191 shows that the subjects spend significantly less time in performing the task for the Cab Distribution system than for the Video Store system (p-value = 0.047 , mean $(C D)=3.36$, mean $(V S)=2.95)$. The subjects also spend more time in using OCL for the Video Store system than for the Cab Distribution system ( $\mathrm{p}$-value $=0.044$, mean $(C D)=1.63$, mean $(V S)=2.16)$. This supports System having a significant effect.

\subsubsection{C-2-II and C-4-II analysis results discussion}

\section{Main effects}

Method has a significant effect in both laboratories: the OCL subjects performed significantly better than the no_OCL subjects, irrespective of the order of the methods used. The performance of the O_N subjects does not improve from Laboratory 2 to Laboratory 4, which is probably due to the absence of OCL Both the high and the low ability N_O subjects' performance improves. Using OCL clearly brings tangible benefit for the Comprehension task in this experiment trial.

The effect of Order of Method is significant, which indicates different extent of the effect of using OCL in the two attempts at the task. However, Method explains about $7 \%$ of the total variation in both attempts. There is no evidence that using OCL has a larger positive effect during the second attempt. Thus, the effect of Order of Method and the learning effect may have confounded with each other. 
Both Ability and System have significant main effects only in Laboratory 4: the high ability subjects perform better than the low ability subjects; and the subjects perform better for the Cab Distribution system than for the Video Store system. It is possible that the subjects are not familiar with the task in Laboratory 2. When more experience is gained, the high ability subjects may have improved faster than the low ability subjects, resulting in better performance in Laboratory 4. Meanwhile, the experience gained may have larger positive effects on simpler systems.

\section{Interaction effects}

There is no significant interaction effect among the factors in this experiment trial.

\subsubsection{Experiment I and Experiment II}

This section compares the results of Experiment I and Experiment II for the Comprehension task. Section 4.2.3.1 describes the analysis results of all the observations (both attempts). Sections 4.2.3.2 and 4.2.3.3 provide comparisons of the two experiment trials for both significant main and interaction effects for each laboratory (each attempt), respectively.

\subsubsection{All observations}

Table 53 summarizes the results of the hypothesis testing which use all the observations from both attempts at the task for both experiments. Statistically, using OCL has 
significantly positive effects in both Experiment I and Experiment II (p-value $=0028$ and 0.0001 , respectively). There is strong evidence that using OCL brings benefits for the Comprehension task.

Table 53. All observations analysis of both experiment trials

\begin{tabular}{|l|l|l|}
\hline Experiment trial & Hypothesis & p-value \\
\hline \multirow{5}{*}{ Experiment I } & $\begin{array}{l}\mathrm{H}_{0}: \mathrm{C}(\mathrm{OCL})=\mathrm{C}(\text { no_OCL }) \\
\text { Experiment II }\end{array}$ & $\mathbf{0 . 0 2 8}$ \\
\cline { 2 - 3 } & $\mathrm{H}_{\mathrm{a}}: \mathrm{C}(\mathrm{OCL})>\mathrm{C}($ no_OCL) & \\
\hline & $\begin{array}{l}\mathrm{H}_{0}: \text { Diff(N_O) }=\text { Diff(O_N) } \\
\mathrm{H}_{\mathrm{a}}: \text { Diff(N_O) }>\text { Diff(O_N) }\end{array}$ & 0.0613 \\
\hline & $\begin{array}{l}\mathrm{H}_{0}: \mathrm{C}(\mathrm{OCL})=\mathrm{C}(\text { no_OCL) } \\
\mathrm{H}_{\mathrm{a}}: \mathrm{C}(\mathrm{OCL})>\mathrm{C}(\text { no_OCL })\end{array}$ & $\mathbf{0 . 0 0 0 1}$ \\
\cline { 2 - 3 } & $\begin{array}{l}\left.\mathrm{H}_{0}: \text { Diff(N_O) }=\text { Diff(O_N }\right) \\
\mathrm{H}_{\mathrm{a}}: \text { Diff(N_O) }>\text { Diff(O_N) }\end{array}$ & $\mathbf{0 . 0 3 9 4}$ \\
\hline
\end{tabular}

In both experiment trials, the difference between the performance of the N_O subjects when OCL is used and not used is larger than that in the O_N subjects, as shown in Table 54. The Order of Method seems to have a weak effect in Experiment I ( $\mathrm{p}$-value $=0.061$ ), and a significant effect in Experiment $\Pi(\mathrm{p}$-value $=0.04)$. Order of Method may have a confounding effect with Method: Using OCL may have larger effect during the second attempt than during the first attempt.

However, it is also possible that the learning effect may confound the effect of Order of Method. The smaller performance difference for the O_N subjects than for the N_O subjects may be because that the learning effect has overcome the positive effect of using OCL. Thus, it cannot be concluded that using OCL during the second attempt has larger effect compared to the first attempt, which may be artificial. Thus, if the effect of Order 
of Method is of interest, it is desirable to eliminate the learning effect in future experiments.

Table 54. Difference of OCL and no_OCL

\begin{tabular}{|c|c|c|c|c|c|c|}
\hline & & & Experimen & & Experimen & \\
\hline & Lab 2 & Lab 4 & $\begin{array}{c}\text { Diff } \\
(\mathrm{OCL}-\text { no_OCL) }\end{array}$ & p-value & $\begin{array}{c}\text { Diff } \\
\text { (OCL-no_OCL) }\end{array}$ & p-value \\
\hline O_N & OCL & No_OCL & $1.43 \%$ & \multirow{2}{*}{0.061} & $4.03 \%$ & \multirow{2}{*}{0.04} \\
\hline N_O & No_OCL & OCL & $11.43 \%$ & & $10.79 \%$ & \\
\hline
\end{tabular}

Further, in Experiment I, only the N_O high ability subjects' performance improves significantly from Laboratory 2 to Laboratory 4; whereas in Experiment II, the performance of the N_O subjects of both the high and the low abilities improves significantly. It is possible that the low ability subjects need more time to mature their OCL knowledge than the high ability subjects. Once the learning period is completed, using OCL is likely to have a positive effect on all the subjects.

\subsubsection{Main effects}

As shown in Table 55, no significant main effects exist in C-2-I, while all the three factors have significant main effects in C-4-I. In Experiment II, Method is the only factor that has significant main effect in both Laboratory 2 and Laboratory 4, whereas Ability and System significantly impact the subjects' performance in Laboratory 4, but not in Laboratory 2. The results of the two experiment trials are consistent, except that Method does not have a significant main effect in C-2-I, in which the subjects are believed to be less experienced in both OCL and UML system analysis. Method explains a large portion 
of the total variation for Laboratory 4 of Experiment I (27\%), which is particularly high. However, it only explains about $7 \%$ of the total variation in both laboratories of Experiment II. It is difficult to explain why OCL has a larger correlation ratio in Experiment I than in Experiment II, which requires more investigation in future experiments.

Table 55. Comparing Experiment I and Experiment II

\begin{tabular}{|c|c|c|c|c|c|c|}
\hline & \multicolumn{3}{|c|}{ Experiment I (two-way ANOVA) } & \multicolumn{3}{|c|}{ Experiment II (three-way ANOVA) } \\
\hline & Significant Effect & p-value & $\mathrm{E}^{2}$ & Significant Effect & p-value & $\mathrm{E}^{2}$ \\
\hline Laboratory 2 & - & - & - & Method & 0.024 & 0.07 \\
\hline \multirow{4}{*}{ Laboratory 4} & $\begin{array}{c}\text { Method } \\
\text { (ANOVA with Ability) }\end{array}$ & 0.003 & 0.27 & Method & 0.015 & 0.07 \\
\hline & $\begin{array}{c}\text { Ability } \\
\text { (ANOVA with Method) }\end{array}$ & 0.035 & 0.12 & Ability & 0.014 & 0.06 \\
\hline & $\begin{array}{c}\text { Method } \\
\text { (ANOVA with System) } \\
\end{array}$ & 0.001 & 0.28 & System & 0.018 & 0.07 \\
\hline & $\begin{array}{c}\text { System } \\
\text { (ANOVA with Method) }\end{array}$ & 0.007 & 0.18 & & & \\
\hline
\end{tabular}

Similarly, in both experiment trials, Ability and System have significant main effects only during the second attempt at the task, which is possibly due to the subjects' unfamiliarity with the task during the first attempt. More experiments could be performed to observe if a trend exists: whether Ability and System become more important for the Comprehension task as more OCL and UML system analysis knowledge is gained. 


\subsubsection{Interaction effects}

No significant interaction effect is observed in any of the laboratories. For the Comprehension task, using OCL has similar effect for high and low ability subjects, and for simpler and more complex systems.

\subsection{Maintenance (M)}

\subsubsection{Experiment I}

\subsubsection{Analyzing data of both laboratories}

\section{All observations analysis}

$\mathrm{H}_{0}: \mathrm{M}(\mathrm{OCL})=\mathrm{M}($ no_OCL $)$

$\mathrm{H}_{\mathrm{a}}: \mathrm{M}(\mathrm{OCL})>\mathrm{M}($ no_OCL $)$

The one-tailed paired t-test is used to test the above hypothesis. It does not yield any significant results for the Maintenance task in Experiment $\mathrm{I}$ ( $\mathrm{p}$-value $=0.159$, mean $(\mathrm{OCL})=36.78 \%$, mean $($ no_OCL $)=31.76 \%)$. Overall, the subjects perform slightly better when OCL is used than when OCL is not used, but the difference is not significant. Since the null hypothesis cannot be rejected at $\alpha=0.05$, there is no evidence that a difference exists in using and not using OCL in this experiment trial. 


\section{Testing the effect of Order of Method}

$\mathrm{H}_{0}: \operatorname{Diff}\left(\mathrm{N} \_\mathrm{O}\right)=\operatorname{Diff}\left(\mathrm{O} \_\mathrm{N}\right)$

$\mathrm{H}_{\mathrm{a}}$ : Diff(N_O) $>$ Diff(O_N)

The one-tailed t-test is used to test the above hypothesis. The N_O subjects perform better when they use OCL compared to when they do not (mean $\left.\left(\operatorname{Diff}\left(\mathrm{N} \_\mathrm{O}\right)\right)=13.75 \%\right)$, while the O_N subjects perform worse $\left(\operatorname{mean}\left(\operatorname{Diff}\left(\mathrm{O} \_\mathrm{N}\right)\right)=-4.32 \%\right)$, as shown in Table 37. The difference is significant $(\mathrm{p}$-value $=0.033$ ). The $\mathrm{O} \mathrm{N}$ subjects' performance improves overtime, which indicates that the learning effect must have overcome the positive effect of using OCL, if present. The N_O subjects' performance improvement is much larger than the O_N subjects by $9.43 \%$. This indicates that a positive effect of using OCL probably exists, at least during the second attempt at the task. This needs confirmation from the second step analysis, where the observations for each attempt are analyzed.

Table 56 Order of Methods applied

\begin{tabular}{|c|c|c|c|}
\hline & Lab 2 & Lab 4 & Diff(OCL - no_OCL) \\
\hline O_N & OCL & No_OCL & $-4.32 \%$ \\
\hline N_O & No_OCL & OCL & $13.75 \%$ \\
\hline
\end{tabular}

\section{Comparing performance between Laboratory 2 and Laboratory 4}

There is a significant overall improvement from Laboratory 2 to Laboratory 4 (p-value $=$ 0.031$, mean $(\operatorname{lab} 2)=30 \%$, mean $(\operatorname{lab} 4)=39 \%)$. Table 57 shows that the high ability subjects experience a weak $(0.05<\mathrm{p}$-value $<0.1)$ improvement in their performance irrespective of the order of the methods used. Using OCL does not seem to have an effect 
on the high ability subjects. On the other hand, the O_N low ability subjects' performance seems to deteriorate over time, while the $\mathrm{N} \_\mathrm{O}$ low ability subjects' performance improves slightly. It seems that using OCL has a positive effect on the low ability subjects. However, none of the abovementioned differences are statistically significant.

Table 57. Comparing the same subject (paired t-test)

\begin{tabular}{|c|c|c|c|c|}
\hline & Ability & Mean (Lab 2) & Mean (Lab 4) & p-value \\
\hline \multirow{2}{*}{ O_N } & High & $28 \%$ & $44.71 \%$ & 0.06 \\
\cline { 2 - 5 } & Low & $25.8 \%$ & $17.4 \%$ & 0.89 \\
\hline \multirow{2}{*}{ N_O } & High & $33.38 \%$ & $51 \%$ & 0.056 \\
\cline { 2 - 5 } & Low & $31.1 \%$ & $40.3 \%$ & 0.202 \\
\hline
\end{tabular}

The survey results suggest the existence of both the learning effect and the effect of using OCL. Table 101 on page 188 shows that the subjects have less difficulties to answer questions in Laboratory 4 than in Laboratory 2 (p-value $=0.012$, mean $(\mathrm{lab} 2)=3.1$, mean $($ lab4 $)=2.55)$. Furthermore, Table 103 on page 188 shows that the subjects perceive using OCL to be more helpful in performing the Maintenance task in Laboratory 4 than in Laboratory 2 (p-value $=0.037$ with mean $(1 \mathrm{ab} 2)=3.23$ and mean $(\mathrm{lab} 4)=2.5$ ).

\subsubsection{Maintenance-2-I}

\section{Testing the hypothesis- M-2-I}

$\mathrm{H}_{0}: \mathrm{M}(\mathrm{OCL})=\mathrm{M}($ no_oCL $)$

$\mathrm{H}_{\mathrm{a}}: \mathrm{M}(\mathrm{OCL})>\mathrm{M}($ no_OCL $)$

The $t$-test yields a p-value of $0.798($ mean $(O C L)=28 \%$, mean $($ no_OCL $)=32 \%)$. Since the null hypothesis cannot be rejected at $\alpha=0.05$, there is no evidence that a difference 
exists between using and not using OCL in this laboratory. Although the OCL subjects perform slightly worse than the no_OCL subjects, there is no evidence that using OCL has negative effect either (corresponding $\mathrm{p}$-value $=0.202$ ).

\section{Two-way ANOVA (Method \& Ability) - M-2-I}

Table 58 shows that neither Method $(\mathrm{p}$-value $=0.414)$ nor Ability $(\mathrm{p}$-value $=0.442)$ has a significant main effect on the subjects' performance for the Maintenance task in Laboratory 2. There is no significant interaction between Method and Ability (p-value = 0.97) either, as shown in Table 59 and Figure 28.

\section{Table 58. Two-way ANOVA (Method and Ability) - M-2-I}

\begin{tabular}{|c|c|c|c|c|c|c|}
\hline Source & DF & Sum of Squares & Mean Square & F Ratio & Prob $>$ F & $E^{2}$ \\
\hline Ability & 1 & 0.01246097 & 0.01246097 & 0.6091 & 0.442 & 0.02 \\
\hline Method & 1 & 0.01408302 & 0.01408302 & 0.6884 & 0.414 & 0.02 \\
\hline Ability*Method & 1 & 0.00003125 & 0.00003125 & 0.0015 & 0.97 & 0.0001 \\
\hline Residual & 28 & 0.57280675 & 0.020457 & & & 0.96 \\
\hline Total & 31 & 0.59922188 & & & & \\
\hline
\end{tabular}

Table 59. Descriptive statistics - M-2-I

\begin{tabular}{|c|c|c|c|c|c|c|}
\cline { 2 - 7 } \multicolumn{1}{c|}{} & \multicolumn{6}{c|}{ Ability } \\
\cline { 2 - 7 } \multicolumn{1}{c|}{ High } & \multicolumn{2}{c|}{ Low } & \multicolumn{2}{c|}{ All } \\
\hline Method & Mean & Size & Mean & Size & Mean & Size \\
\hline OCL & $30 \%$ & 8 & $26 \%$ & 7 & $28 \%$ & 15 \\
\hline No_OCL & $34 \%$ & 9 & $30 \%$ & 8 & $32 \%$ & 17 \\
\hline All Methods & $32 \%$ & 17 & $28 \%$ & 15 & $30 \%$ & 32 \\
\hline
\end{tabular}


Figure 28. Graph of means - M-2-I

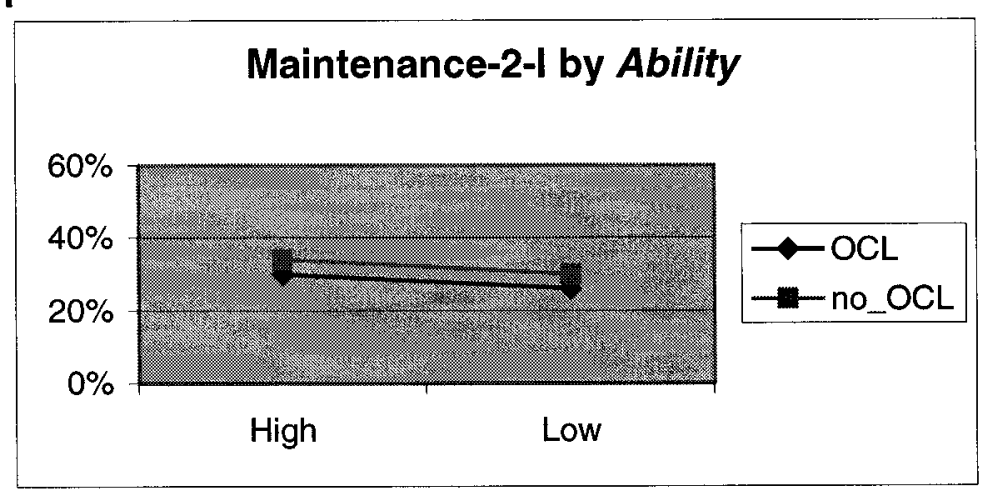

Two-way ANOVA (Method \& System) - M-2-I

Table 60 shows that only System has a significant main effect, explaining $26 \%$ of the total variation $\left(E^{2}=0.26\right)$. Table 61 and Figure 29 show that the subjects perform better for the Cab Distribution system than for the Video Store system. There is no significant interaction between Method and System (p-value $=0.742$ ).

Table 60. Two-way ANOVA (Method \& System) - M-2-I

\begin{tabular}{|c|c|c|c|c|c|c|}
\hline Source & DF & Sum of Squares & Mean Square & F Ratio & Prob $>$ F & $E^{2}$ \\
\hline System & 1 & 0.15343412 & 0.15343412 & 10.0495 & $\mathbf{0 . 0 0 4}$ & 0.26 \\
\hline Method & 1 & 0.01414278 & 0.01414278 & 0.9263 & 0.344 & 0.02 \\
\hline System*Method & 1 & 0.0016857 & 0.0016857 & 0.1104 & 0.742 & 0.003 \\
\hline Residual & 28 & 0.42749782 & 0.015268 & & & 0.71 \\
\hline Total & 31 & 0.59922188 & & & & \\
\hline
\end{tabular}

Table 61. Descriptive Statistics- M-2-I

\begin{tabular}{|c|c|c|c|c|c|c|}
\cline { 2 - 7 } \multicolumn{1}{c|}{} & \multicolumn{6}{c|}{ System } \\
\cline { 2 - 7 } \multicolumn{1}{c|}{} & \multicolumn{2}{c|}{ CD } & \multicolumn{2}{c|}{ VS } & \multicolumn{2}{c|}{ All } \\
\hline Method & Mean & Size & Mean & Size & Mean & Size \\
\hline OCL & $35 \%$ & 7 & $22 \%$ & 8 & $28 \%$ & 15 \\
\hline No_OCL & $40 \%$ & 8 & $25 \%$ & 9 & $32 \%$ & 17 \\
\hline All Methods & $38 \%$ & 15 & $24 \%$ & 17 & $30 \%$ & 32 \\
\hline
\end{tabular}


Figure 29. Graph of Means - M-2-I

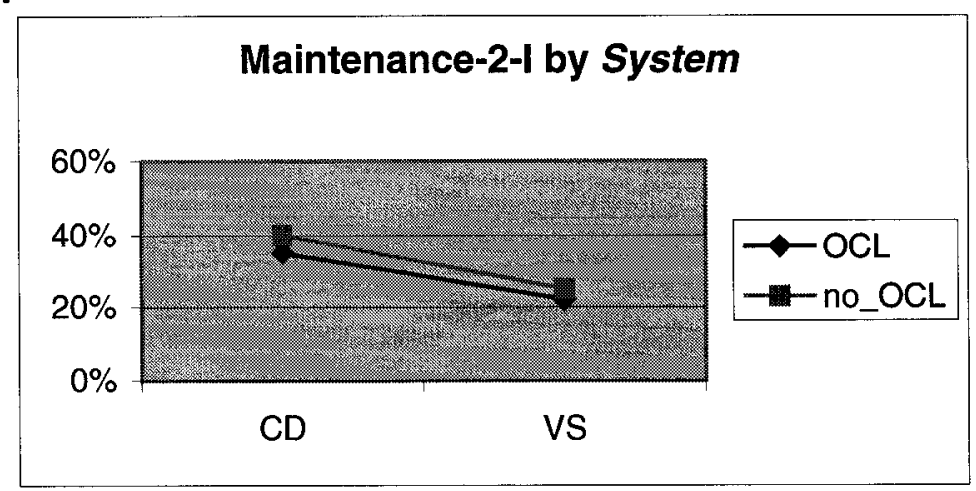

Combining two ANOVA analyses - M-2-I

Combing both two-way ANOVA results, System is the only factor that has a significant main effect. However, the survey results do not provide additional support, shown in Table 97 on page 186.

\subsubsection{Maintenance-4-I}

\section{Testing the hypothesis- M-4-I}

$\mathrm{H}_{0}: \mathrm{M}(\mathrm{OCL})=\mathrm{M}($ no_OCL $)$

$\mathrm{H}_{\mathrm{a}}: \mathrm{M}(\mathrm{OCL})>\mathrm{M}($ no_OCL $)$

The $t$-test yields a p-value of 0.024 (mean $(\mathrm{OCL})=46 \%$, mean $($ no_OCL) $=31 \%$ ). Since the null hypothesis is rejected at $\alpha=0.05$, using OCL has a significant positive effect on the dependent variable Maintenance in this laboratory.

Two-way ANOVA (Method \& Ability) - M-4-I

Table 62 shows that both Ability and Method have significant main effects (pvalue $($ Ability $)=0.006, \mathrm{p}$-value $($ Method $)=0.029)$, explaining $22 \%\left(\mathrm{E}^{2}=0.22\right)$ and $13 \%$ $\left(E^{2}=0.13\right)$ of the total variation, respectively, both of which are particularly high. Table 
63 and Figure 30 show that using OCL has a positive effect on the subjects' overall performance, and that the high ability subjects perform better than the low ability subjects. Although Table 63 and Figure 30 suggest an interaction between Method and Ability, it is not significant ( $\mathrm{p}$-value $=0.202$ ).

Table 62. Two-way ANOVA (Method \& Ability) - M-4-I

\begin{tabular}{|c|c|c|c|c|c|c|}
\hline Source & DF & Sum of Squares & Mean Square & F Ratio & Prob > F & E2 \\
\hline Ability & 1 & 0.255256 & 0.255256 & 9.0546 & $\mathbf{0 . 0 0 6}$ & 0.22 \\
\hline Method & 1 & 0.150416 & 0.150416 & 5.3357 & $\mathbf{0 . 0 2 9}$ & 0.13 \\
\hline Ability*Method & 1 & 0.048357 & 0.048357 & 1.7154 & 0.202 & 0.04 \\
\hline Residual & 25 & 0.704764 & 0.028191 & & & 0.61 \\
\hline Total & 28 & 1.159742 & & & & \\
\hline
\end{tabular}

Table 63. Descriptive statistics - M-4-I

\begin{tabular}{|c|c|c|c|c|c|c|}
\hline \multirow[b]{3}{*}{ Method } & \multicolumn{6}{|c|}{ Ability } \\
\hline & \multicolumn{2}{|c|}{ High } & \multicolumn{2}{|c|}{ Low } & \multicolumn{2}{|c|}{ All } \\
\hline & Mean & Size & Mean & Size & Mean & Size \\
\hline $\mathrm{OCL}$ & $51 \%$ & 8 & $40 \%$ & 7 & $46 \%$ & 15 \\
\hline No_OCL & $45 \%$ & 7 & $18 \%$ & 7 & $31 \%$ & 14 \\
\hline All Methods & $48 \%$ & 15 & $29 \%$ & 14 & $39 \%$ & 29 \\
\hline
\end{tabular}

Figure 30. Graph of means - M-4-I

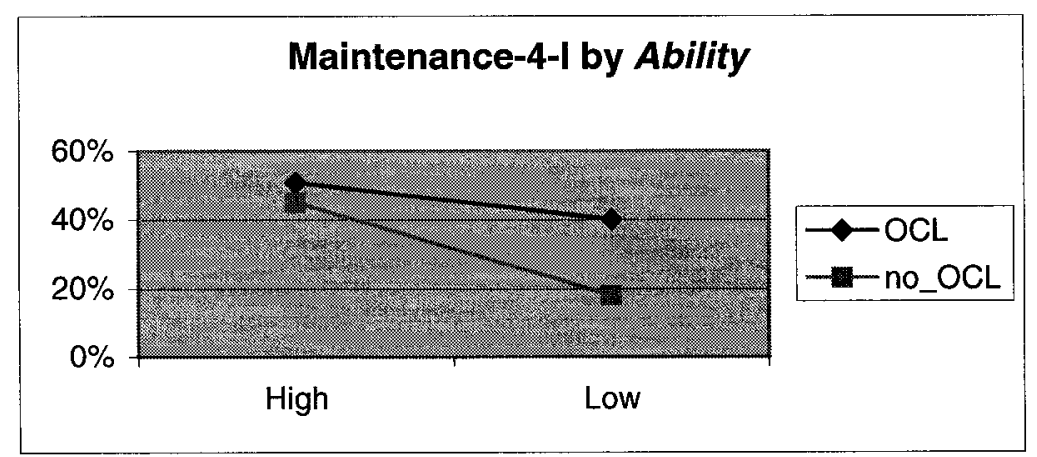


Two-way ANOVA (Method \& System) - M-4-I

Table 64 shows that both System and Method have significant main effects $(\mathrm{p}$-value $($ System $)<0.0001, \mathrm{p}$-value $($ Method $)=0.013)$, explaining $47 \%\left(\mathrm{E}^{2}=0.47\right)$ and $11 \%\left(E^{2}=0.11\right)$ of the total variation, respectively. The effects can be observed from Table 65 and Figure 31: using OCL has a positive effect on the subjects' overall performance, and the subjects perform better for the Cab Distribution system than for the Video Store system. There is no significant interaction between System and Method.

Table 64. Two-way ANOVA (Method and System) - M-4-I

\begin{tabular}{|c|c|c|c|c|c|c|}
\hline Source & DF & Sum of Squares & Mean Square & F Ratio & Prob > F & E2 \\
\hline System & 1 & 0.546971 & 0.546971 & 30.8789 & $<.0001$ & 0.47 \\
\hline Method & 1 & 0.126156 & 0.126156 & 7.1221 & $\mathbf{0 . 0 1 3}$ & 0.11 \\
\hline System*Method & 1 & 0.008127 & 0.008127 & 0.4588 & 0.504 & 0.01 \\
\hline Residual & 25 & 0.442836 & 0.017713 & & & 0.38 \\
\hline Total & 28 & 1.159742 & & & & \\
\hline
\end{tabular}

Table 65. Descriptive statistics- M-4-I

\begin{tabular}{|c|c|c|c|c|c|c|}
\hline \multirow[b]{3}{*}{ Method } & \multicolumn{6}{|c|}{ System } \\
\hline & \multicolumn{2}{|c|}{$\mathrm{CD}$} & \multicolumn{2}{|c|}{ VS } & \multicolumn{2}{|c|}{ All } \\
\hline & Mean & Size & Mean & Size & Mean & Size \\
\hline $\mathrm{OCL}$ & $59 \%$ & 9 & $27 \%$ & 6 & $46 \%$ & 15 \\
\hline No_OCL & $42 \%$ & 8 & $17 \%$ & 6 & $31 \%$ & 14 \\
\hline All Methods & $51 \%$ & 17 & $22 \%$ & 12 & $39 \%$ & 29 \\
\hline
\end{tabular}


Figure 31. Graph of Means - M-4-I

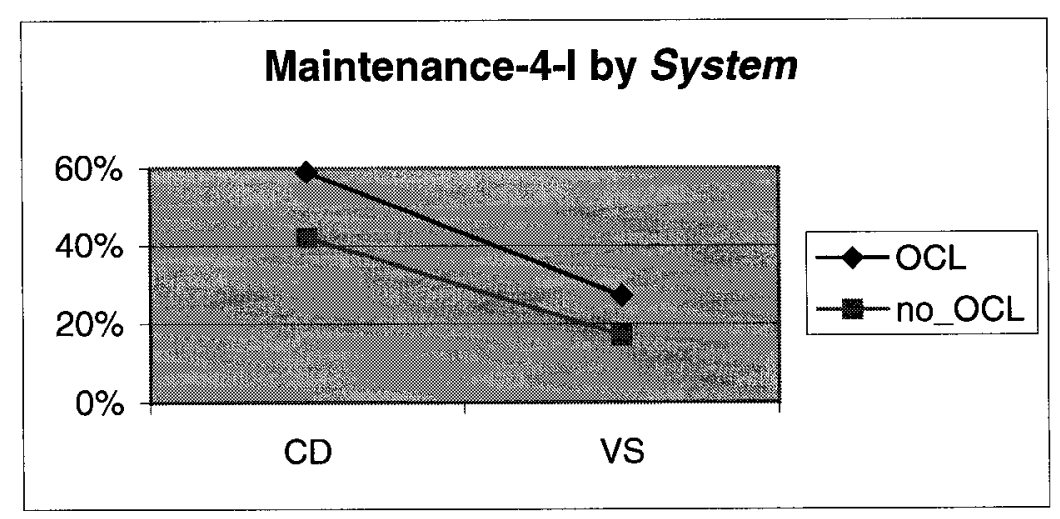

Combining two ANOVA analyses - M-4-I

Combining both two-way ANOVA results, all the three factors, System, Ability, and Method, have significant main effects in this laboratory. There is no significant interaction effect among the factors.

Other than the survey difference already discussed for the Comprehension task: the OCL subjects feel less time pressure, and the OCL subjects also consider the Maintenance questions to be easier to answer compared to the no_OCL subjects ( $\mathrm{p}$-value $=0.01$, mean $(\mathrm{OCL})=2.8$, mean $($ no_OCL $)=2$ ), as shown in Table 98 on page 186. This supports Method having a significant main effect.

Compared to the Video Store system, the subjects also perceive using OCL to be more helpful for the Cab Distribution system $(\mathrm{p}$-value $=0.031$, mean $(\mathrm{CD})=1.63$, mean $(\mathrm{VS})=$ 2.5 ), and the Cab Distribution system easier to understand ( $\mathrm{p}$-value $=0.001$, mean $(\mathrm{CD})=$ 1.88, mean $(\mathrm{VS})=3.33$ ), as shown in Table 102 on page 187. This supports System 
having a significant main effect. There is no evidence provided by the survey results for Ability having a significant main effect (Table 100 on page 187).

\subsubsection{M-2-I and M-4-I}

\section{Main effects}

System is the only factor that has a significant main effect in both Laboratory 2 and Laboratory 4.

Method has a significant main effect only in Laboratory 4, which may be due to the subjects' inexperience with using OCL the first time they perform the Maintenance task. Once more OCL knowledge is gained, Method is likely to make a difference. The learning effect exists, as all the subjects' performance improves from Laboratory 2 to Laboratory 4, especially for the high ability subjects. The improvement for the N_O subjects is larger than that of the O_N subjects, which suggests that the effect of using OCL exists, especially for the low ability subjects.

Ability has a significant main effect only in Laboratory 4: the high ability subjects perform better than the low ability subjects. It is possible that the high ability subjects gain more UML system analysis knowledge than the low ability subjects in the same period of time. This may result in a faster improvement in the performance of the high ability subjects than that of the low ability subjects. 


\section{Interaction effects}

There is no significant interaction effect among factors in this experiment trial.

\subsubsection{Experiment II}

\subsubsection{Analyzing data of both laboratories}

\section{All observations analysis}

$\mathrm{H}_{0}: \mathrm{M}(\mathrm{OCL})=\mathrm{M}($ no_OCL $)$

$\mathrm{H}_{\mathrm{a}}: \mathrm{M}(\mathrm{OCL})>\mathrm{M}($ no_OCL $)$

The one-tailed paired t-test is used to test the above hypothesis. The subjects perform significantly better when OCL is used than when OCL is not used for the Maintenance task in Experiment II (p-value $=0.003$, mean $(\mathrm{OCL})=41.37 \%$, mean (no_OCL) $=$ $33.25 \%$ ). Since the null hypothesis is rejected at $\alpha=0.05$, it can be concluded that using OCL has a significant positive effect on the dependent variable Maintenance in this experiment trial.

\section{Testing order of Method of diff(OCL - no_OCL)}

$\mathrm{H}_{0}: \operatorname{Diff}\left(\mathrm{N} \_\mathrm{O}\right)=\operatorname{Diff}\left(\mathrm{O} \_\mathrm{N}\right)$

$\mathrm{H}_{\mathrm{a}}: \operatorname{Diff}\left(\mathrm{N} \_\mathrm{O}\right)>\operatorname{Diff}\left(\mathrm{O} \_\mathrm{N}\right)$

The one-tailed t-test is used to test the above hypothesis. The N_O subjects perform better when they apply OCL than when they do not $\left(\right.$ mean $\left(\right.$ Diff $\left.\left.\left(\mathrm{N} \_\mathrm{O}\right)\right)=9.82 \%\right)$, whereas the O_N subjects also perform better $\left(\right.$ mean $\left.\left(\operatorname{Diff}\left(\mathrm{O} \_\mathrm{N}\right)\right)=6.06 \%\right)$, as shown in Table 66 . The difference is not statistically significant ( $p$-value $=0.258$ ). This suggests that using 
OCL first or later does not have an effect. Since Maintenance is the last task, after working with OCL during the Defect Detection and the Comprehension tasks, the subjects may have gained enough experience using OCL effectively the very first time they perform the Maintenance task in Laboratory 2. The learning effect, if present, does not seem to overcome the positive effect of using OCL for the O_N subjects.

Table 66 Order of Methods applied

\begin{tabular}{|c|c|c|c|}
\hline & Lab 2 & Lab 4 & Diff(OCL - no_OCL) \\
\hline O_N & OCL & No_OCL & $6.06 \%$ \\
\hline N_O & No_OCL & OCL & $9.82 \%$ \\
\hline
\end{tabular}

\section{Comparing performance between Laboratory 2 and Laboratory 4}

There is no significant overall performance difference between the two laboratories ( $p$ value $=0.199$, mean $(l a b 2)=36 \%$, mean $(l a b 4)=38 \%)$.

However, Table 67 shows that the N_O low ability subjects have a significant improvement in performance from Laboratory 2 to Laboratory 4 ( $p$-value $=0.003$ ), which demonstrates a significant positive effect of using OCL for the low ability subjects. It is possible that using OCL may have a larger effect on the low ability subjects than on the high ability subjects for the Maintenance task.

Table 67. Comparing the same subject (paired t-test)

\begin{tabular}{|c|c|c|c|c|}
\hline & Ability & Mean (Lab 2) & Mean (Lab 4) & p-value \\
\hline \multirow{2}{*}{ O_N } & High & $40.5 \%$ & $36.69 \%$ & 0.721 \\
\cline { 2 - 5 } & Low & $42.88 \%$ & $34.71 \%$ & 0.867 \\
\hline \multirow{2}{*}{ N_O } & High & $36 \%$ & $41.67 \%$ & 0.156 \\
\cline { 2 - 5 } & Low & $27.1 \%$ & $40.48 \%$ & $\mathbf{0 . 0 0 3}$ \\
\hline
\end{tabular}


Table 110 on page 191 shows that the subjects have significantly less problems to understand the system in Laboratory 4 than in Laboratory 2 (p-value $=0.02$, mean(lab2) $=2.68$, mean $(\mathrm{lab} 4)=2.43)$. This indicates the existence of the learning effect . However, Table 112 on page 191 shows that although the subjects spend significantly more time to use OCL $(p$-value $=0.039$, mean $(\mathrm{lab} 2)=1.62$, mean $(\mathrm{lab} 4)=2.04)$, in Laboratory 4 than in Laboratory 2, they do not find it more useful ( $\mathrm{p}$-value $=0.314$, mean $(\operatorname{lab} 2)=2.59$, mean $($ lab4) $=2.47)$. This does not seem to be consistent with the abovementioned laboratory observations, and needs further investigation in future experiments, possibly through post-lab interviews.

\subsubsection{Maintenance-2-II}

\section{Testing the hypothesis - M-2-II}

$\mathrm{H}_{0}: \mathrm{M}(\mathrm{OCL})=\mathrm{M}($ no_OCL $)$

$\mathrm{H}_{\mathrm{a}}: \mathrm{M}(\mathrm{OCL})>\mathrm{M}($ no_OCL $)$

The $t$-test yields a p-value of $0.003($ mean $(\mathrm{OCL})=40 \%$, mean $($ no_OCL $)=31 \%)$. Since the null hypothesis is rejected at $\alpha=0.05$, it can be concluded that using OCL has a significant positive effect on the dependent variable Maintenance in this laboratory. This confirms the results, discussed above, that using OCL has positive effect from the very first attempt at the task.

\section{Three-way ANOVA (Method, Ability \& System)}

Table 68 shows that both Method and System have significant main effects in this laboratory ( $p$-value $=0.0002$ and $<0.0001$, respectively). Method explains $9 \%$ of the total 
variation $\left(E^{2}=0.09\right)$, and System explains $39 \%$ of the total variation $\left(E^{2}=0.39\right)$. As shown in Table 68, Figure 32 and Figure 33, using OCL has a positive effect on the subjects' overall performance, and the subjects perform better for the Cab Distribution system than for the Video Store system.

Although Ability does not have a significant main effect $(\mathrm{p}$-value $=0.144)$, it interacts with Method significantly (p-value $=0.05$ ). As shown in Table 69 and Figure 33, using OCL benefits the low ability subjects more than the high ability subjects. There is also a significant interaction between System and Method (p-value $=0.004$ ). As shown in Table 69 and Figure 32, using OCL has more benefit for the Cab Distribution system than for the Video Store system.

Table 68. Three-way ANOVA (Method, Ability \& System) - M-2-II

\begin{tabular}{|c|c|c|c|c|c|c|}
\hline Source & DF & Sum of Squares & Mean Square & F Ratio & Prob $>$ F & $\mathrm{E}^{2}$ \\
\hline System & 1 & 0.71581679 & 0.71581679 & 67.352 & $<.0001$ & 0.39 \\
\hline Ability & 1 & 0.0231627 & 0.0231627 & 2.1794 & 0.144 & 0.01 \\
\hline Method & 1 & 0.16907168 & 0.16907168 & 15.9081 & $\mathbf{0 . 0 0 0 2}$ & 0.09 \\
\hline Ability*System & 1 & 0.02404459 & 0.02404459 & 2.2624 & 0.137 & 0.01 \\
\hline Method*System & 1 & 0.09447054 & 0.09447054 & 8.8888 & $\mathbf{0 . 0 0 4}$ & 0.05 \\
\hline Ability*Method & 1 & 0.04244559 & 0.04244559 & 3.9938 & $\mathbf{0 . 0 5}$ & 0.02 \\
\hline Ability*Method *System & 1 & 0.03920656 & 0.03920656 & 3.689 & 0.059 & 0.02 \\
\hline Residual & 69 & 0.733332 & 0.010628 & & & 0.40 \\
\hline Total & 76 & 1.8315247 & & & & \\
\hline
\end{tabular}


Table 69. Descriptive statistics- M-2-II

\begin{tabular}{|c|c|c|c|c|}
\hline Method & Ability & System & Mean & Size \\
\hline \multirow{4}{*}{$\begin{array}{l}\text { OCL } \\
\text { Mean:40\% } \\
\text { Size: } 39\end{array}$} & \multirow{2}{*}{$\begin{array}{l}\text { High } \\
\text { Mean:41\%, Size: } 18\end{array}$} & $C D$ & $53.00 \%$ & 9 \\
\hline & & VS & $29.67 \%$ & 9 \\
\hline & \multirow{2}{*}{$\begin{array}{l}\text { Low } \\
\text { Mean:40\%, Size: } 21\end{array}$} & $C D$ & $55.22 \%$ & 9 \\
\hline & & VS & $27.75 \%$ & 12 \\
\hline \multirow{4}{*}{$\begin{array}{l}\text { no_OCL } \\
\text { Mean: } \\
31 \% \\
\text { Size: } 40\end{array}$} & \multirow{2}{*}{$\begin{array}{l}\text { High } \\
\text { Mean:35\%, Size: } 19\end{array}$} & $\mathrm{CD}$ & $45.78 \%$ & 9 \\
\hline & & VS & $25.30 \%$ & 10 \\
\hline & \multirow{2}{*}{$\begin{array}{l}\text { Low } \\
\text { Mean:27\%, Size:21 }\end{array}$} & $\mathrm{CD}$ & $29.20 \%$ & 10 \\
\hline & & VS & $25.18 \%$ & 11 \\
\hline
\end{tabular}

Figure 32. Graph of Means by Method and Ability - M-2-II
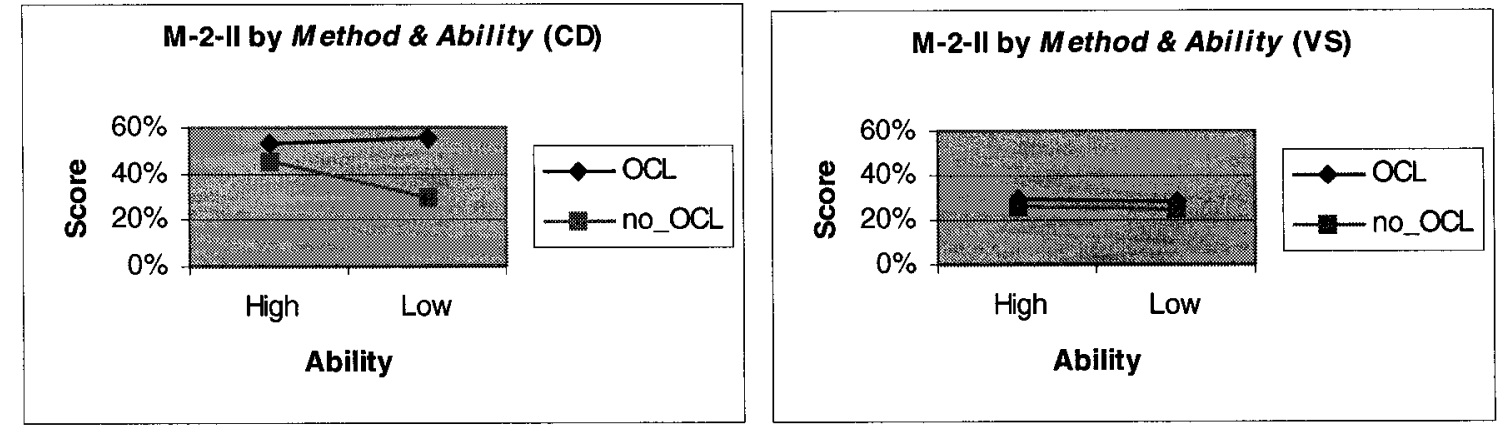

Figure 33. Graph of Means by Method and System - M-2-II
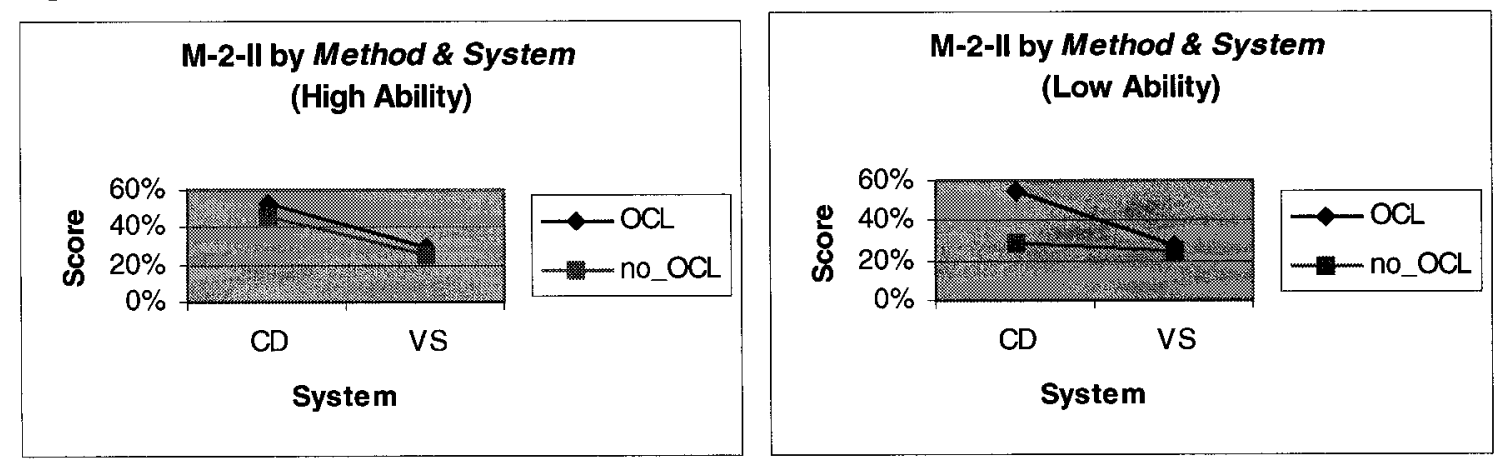

However, the survey results do not provide supporting evidence for Method having a significant main effect: there is no difference between the survey responses from the OCL and the no_OCL subjects, as shown in Table 105 on page 189. 
Although the subjects perform better for the Cab Distribution system than for the Video Store system, Table 106 on page 189 shows that the subjects consider that it is significantly more difficult to complete the Maintenance task for the Cab Distribution system than for the Video Store system (p-value $=0.003$, $\operatorname{mean}(C D)=3.23$, mean(VS)=2.6). The subjects also need more time to perform the task for the $\mathrm{Cab}$ Distribution system than for the Video Store system ( $\mathrm{p}$-value $=0.026$, mean $(C D)=2.82$, mean $(\mathrm{CS})=3.4$ ). These contradict the laboratory observations, and cannot be interpreted in this experiment. Further, the survey results do not provide additional support regarding using OCL having a larger positive effect on the Cab Distribution system than on the Video Store system, other than less time being spent on using OCL for the Cab Distribution system than for the Video Store system $(\mathrm{p}$-value $=0.019$, mean $(C D)=1.33$, mean $(V S)=1.89$ ), as shown in Table 106 on page 189 . More survey questions or postlab interviews may clarify the confusions in future experiments.

\subsubsection{Maintenenace-4-II}

\section{Testing the hypothesis}

$\mathrm{H}_{0}: \mathrm{M}(\mathrm{OCL})=\mathrm{M}($ no_OCL $)$

$\mathrm{H}_{\mathrm{a}}: \mathrm{M}(\mathrm{OCL})>\mathrm{M}$ (no_OCL)

The $t$-test yields a p-value of $0.043(\operatorname{mean}(\mathrm{OCL})=41 \%$, mean $($ no_OCL $)=35 \%)$. Since the null hypothesis is rejected at $\alpha=0.05$, it can be concluded that using OCL has a significant positive effect on the dependent variable Maintenance in this laboratory. 


\section{Three-way ANOVA (Method, Ability \& System)}

Both Method and System have significant main effects (p-value $=0.023$ and $<0.0001$, respectively), but not Ability (p-value $=0.291$ ), as shown in Table 70. Method and System explain $3.6 \%$ and $49.11 \%$ of the total variation, respectively. As shown in Table 71 , Figure 34 and Figure 35, using OCL has a positive effect on the subjects' overall performance, and the subjects perform better for the Cab Distribution system than for the Video Store system. There is no interaction among the three factors.

Table 70. Three-way ANOVA (Method Ability \& System) - M-4-II

\begin{tabular}{|c|c|c|c|c|c|c|}
\hline Source & DF & Sum of Squares & Mean Square & F Ratio & Prob $>$ F & $\mathrm{E}^{2}$ \\
\hline System & 1 & 0.83884456 & 0.83884456 & 74.061 & $<.0001$ & 0.49 \\
\hline Ability & 1 & 0.01284929 & 0.01284929 & 1.1345 & 0.291 & 0.01 \\
\hline Method & 1 & 0.06149857 & 0.06149857 & 5.4297 & $\mathbf{0 . 0 2 3}$ & 0.05 \\
\hline Ability*System & 1 & 0.02019945 & 0.02019945 & 1.7834 & 0.187 & 0.01 \\
\hline Method*System & 1 & 0.00028742 & 0.00028742 & 0.0254 & 0.874 & 0.0002 \\
\hline Ability*Method & 1 & 0.00131911 & 0.00131911 & 0.1165 & 0.734 & 0.001 \\
\hline Ability*Method *System & 1 & 0.00122231 & 0.00122231 & 0.1079 & 0.744 & 0.001 \\
\hline Residual & 65 & 0.7362162 & 0.011326 & & & 0.43 \\
\hline Total & 72 & 1.708063 & & & & \\
\hline
\end{tabular}


Table 71. Descriptive statistics - M-4-II

\begin{tabular}{|c|c|c|c|c|c|}
\hline Method & Ability & System & Mean & Size & Stdev \\
\hline \multirow{4}{*}{$\begin{array}{l}\text { OCL } \\
\text { Mean: } 41 \% \\
\text { Size: } 39\end{array}$} & \multirow{2}{*}{$\begin{array}{l}\text { High } \\
\text { Mean:42\%, Size: } 18\end{array}$} & $\mathrm{CD}$ & $50.56 \%$ & 9 & 0.11 \\
\hline & & VS & $32.78 \%$ & 9 & 0.07 \\
\hline & \multirow{2}{*}{$\begin{array}{l}\text { Low } \\
\text { Mean:40\%, Size:21 }\end{array}$} & $\mathrm{CD}$ & $52.91 \%$ & 11 & 0.14 \\
\hline & & VS & $26.80 \%$ & 10 & 0.09 \\
\hline \multirow{4}{*}{$\begin{array}{l}\text { no_OCL } \\
\text { Mean: } 35 \% \\
\text { Size: } 34\end{array}$} & \multirow{2}{*}{$\begin{array}{l}\text { High } \\
\text { Mean:37\%, Size: } 16\end{array}$} & $\mathrm{CD}$ & $46.00 \%$ & 8 & 0.11 \\
\hline & & VS & $27.38 \%$ & 8 & 0.07 \\
\hline & \multirow{2}{*}{$\begin{array}{l}\text { Low } \\
\text { Mean:33\%, Size:18 }\end{array}$} & $\mathrm{CD}$ & $45.00 \%$ & 9 & 0.14 \\
\hline & & VS & $21.33 \%$ & 9 & 0.1 \\
\hline
\end{tabular}

Figure 34. Graph of Means by Method and Ability - M-4-II
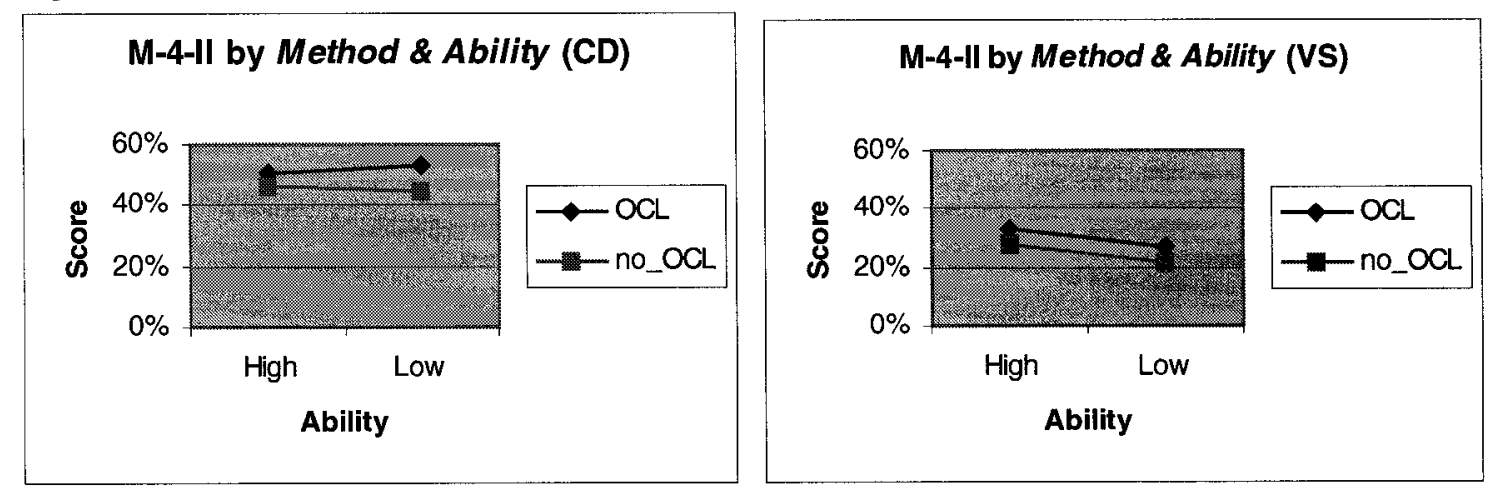

Figure 35. Graph of Means by Method and System - M-4-II
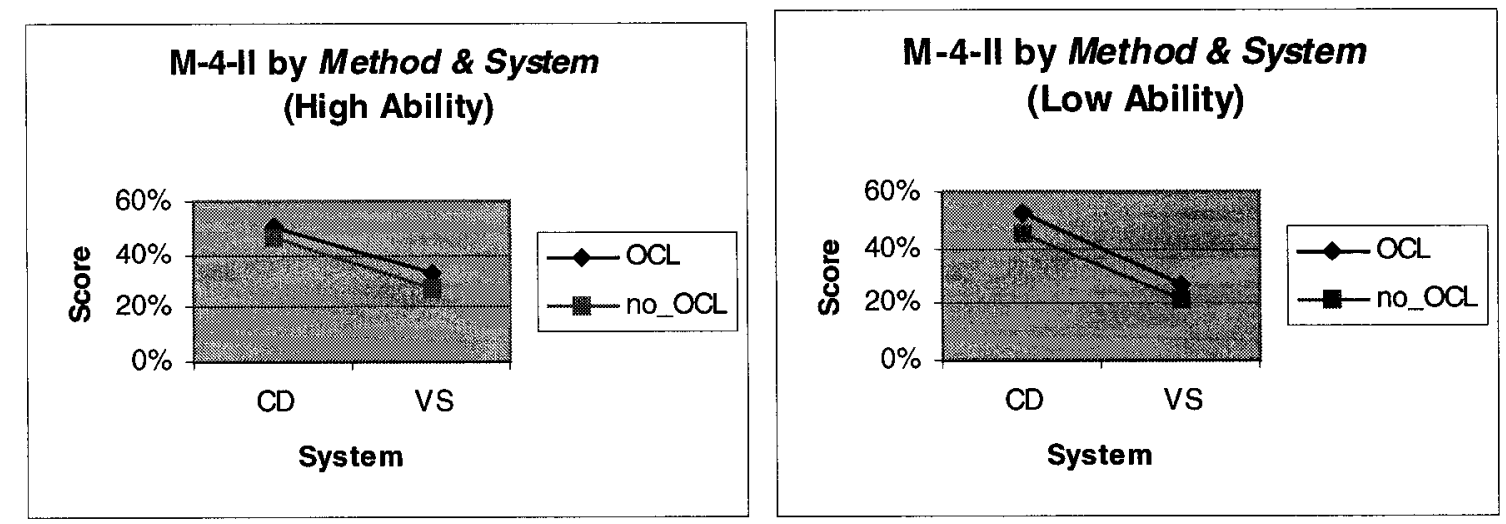

Other than the subjects spending significantly less time in reading and using OCL for the Cab Distribution system than for the Video Store system (p-value $=0.044$, mean $(C D)=$ 1.63 , mean $(\mathrm{VS})=2.16$ ), as shown in Table 107 on page 190 and Table 111 on page 191 , 
there is no other difference in the Maintenance task specific questions between groups. Thus, no further evidence is provided by the survey results to support Method and System having significant main effects.

\subsubsection{M-2-II and M-4-II analysis results discussion}

\section{Main effects}

Method has a significant main effect in both laboratories in this experiment trial: the subjects perform significantly better when OCL is used than when OCL is not used. The subjects seem to use OCL effectively from the first attempt at the task, which may be due to the experience gained in the preceding tasks. System also has a significant main effect in both laboratories: the subjects perform significantly better for the Cab Distribution system than for the Video Store system. However, the significant effects of the factors do not find supporting evidence in the survey responses. More survey questions and/or postlab interviews would be desirable for clarification in future experiments.

\section{Interaction effects}

Significant interactions between Method \& Ability and Method \& System are observed in Laboratory 2, but not in Laboratory 4 of Experiment II. The total variation explained by the interactions, in Laboratory 2, is rather small (2.19\% for Method * Ability, and $4.92 \%$ for Method * System). The impact of Method is smaller in Laboratory 4 than in Laboratory $2\left(E^{2}(\operatorname{lab} 2)=0.09, E^{2}(1 a b 4)=0.04\right)$. The absence of interactions may be due to the decrease of the effect of Method in Laboratory 4. It is difficult to explain why using 
OCL has a smaller impact in Laboratory 4 than in Laboratory 2. More study is required in future experiments to determine whether there is a trend that using OCL may have smaller effect for the Maintenance task over time or not.

\subsubsection{Experiment I and Experiment II}

This section compares the results from Experiment I and Experiment II for the Maintenance task. Section 4.3.3.1 describes the analysis results of all the observations (two attempts). Sections 4.3.3.2 and 4.3.3.3 provide comparisons of the two experiment trials with respect to significant main and interaction effects for each laboratory, respectively.

\subsubsection{All observations}

Table 72 summarizes the results of the hypothesis testing of all observations for both experiment trials for the Maintenance task. Statistically, there is no difference in using and not using OCL in Experiment I, while using OCL has a significant positive effect on the subjects' performance in Experiment II. This can be explained by the fact that the subjects of Experiment II have received more prior training in OCL and the tasks than those of Experiment I: in Experiment I, the subjects are not yet prepared to use OCL in maintaining UML system analysis documents; while in Experiment II, the subjects may be better prepared because of the extra OCL practice received. Unfortunately, the survey results do not provide any supporting evidence. 
Table 72. All observations analysis of both experiment trials

\begin{tabular}{|l|l|c|}
\hline Experiment trial & Hypothesis & p-value \\
\hline \multirow{5}{*}{ Experiment I } & $\begin{array}{l}\mathrm{H}_{0}: \mathrm{M}(\mathrm{OCL})=\mathrm{M}(\text { no_OCL }) \\
\mathrm{H}_{\mathrm{a}}: \mathrm{M}(\mathrm{OCL})>\mathrm{M}(\text { no_OCL })\end{array}$ & 0.159 \\
\cline { 2 - 3 } & $\begin{array}{l}\left.\mathrm{H}_{0}: \text { Diff(N_O) }=\text { Diff(O_N }\right) \\
\left.\mathrm{H}_{\mathrm{a}}: \text { Diff(N_O) }>\text { Diff(O_N }\right)\end{array}$ & $\mathbf{0 . 0 3 3}$ \\
\hline \multirow{5}{*}{ Experiment II } & $\begin{array}{l}\mathrm{H}_{0}: \mathrm{M}(\mathrm{OCL})=\mathrm{M}(\text { no_OCL }) \\
\mathrm{H}_{\mathrm{a}}: \mathrm{M}(\mathrm{OCL})>\mathrm{M}(\text { no_OCL })\end{array}$ & $\mathbf{0 . 0 0 3}$ \\
\cline { 2 - 3 } & $\begin{array}{l}\left.\mathrm{H}_{0}: \text { Diff(N_O }\right)=\text { Diff }(\mathrm{O} \text { ON }) \\
\left.\left.\mathrm{H}_{\mathrm{a}}: \text { Diff(N_O }\right)>\text { Diff(O_N }\right)\end{array}$ & 0.258 \\
\hline
\end{tabular}

Order of Method has a significant effect in Experiment I: the difference between the performance of the N_O subjects when they use OCL and not use OCL is larger than that of the O_N subjects ( $\mathrm{p}$-value $=0.033$ ); while in Experiment II, Order of Method does not have any effect ( $\mathrm{p}$-value $=0.258$ ). Table 73 further shows that using OCL does not have an effect on the subjects' performance in Laboratory 2 of Experiment I, while helps the subjects' performance in Laboratory 4 of Experiment I. On the other hand, in Experiment II, using OCL no longer has any negative impact even in Laboratory 2 . Recall that Maintenance is the last task of all tasks performed, and the subjects of Experiment II have much longer time to mature their OCL knowledge than their fellow subjects of Experiment I. It is possible that the subjects of Experiment II have gained enough OCL knowledge that using OCL first or later does not matter any more. 
Table 73. Difference of OCL and no_OCL

\begin{tabular}{|c|c|c|c|c|c|c|}
\hline & & & \multicolumn{2}{|c|}{ Experiment I } & \multicolumn{2}{|c|}{ Experiment II } \\
\cline { 1 - 1 } & Lab 1 & Lab 3 & $\begin{array}{c}\text { Diff } \\
\text { (OCL - no_OCL) }\end{array}$ & p-value & $\begin{array}{c}\text { Diff } \\
\text { (OCL - no_OCL) }\end{array}$ & p-value \\
\hline O_N & OCL & No_OCL & $-4.32 \%$ & \multirow{2}{*}{$\mathbf{0 . 0 3 3}$} & $6.06 \%$ & \multirow{2}{*}{$\mathbf{0 . 2 5 8}$} \\
\hline N_O & No_OCL & OCL & $13.75 \%$ & & $9.82 \%$ & \\
\hline
\end{tabular}

\subsubsection{Main effects}

Table 74 compares the significant main effects and their correlation ratios for Experiment I and Experiment II.

Method has significant main effects in all laboratories, except M-2-I, where the subjects' OCL knowledge is believed to be less than in other laboratories. This is consistent with the results of the Comprehension task.

Ability has a significant main effect in M-4-I only. It does not have any significant main effect in the Experiment II at all. The absence of Ability's effect in M-2-I may be explained by the subjects' unfamiliarity to the task. After a learning period, the high ability subjects may have improved faster than the low ability subjects, which explains Ability's effect in M-4-I. On the other hand, in Experiment II, it is possible that the low ability subjects have caught up with the high ability subjects with respect to UML and OCL knowledge after having gone through two preceding tasks (Defect Detection, Comprehension), which may result in the absence of Ability's effect in both laboratories. The above interpretation needs to be investigated further through future experiments. 
In all the laboratories, System has significant main effects with the highest correlation ratios $\left(0.26 \leq \mathrm{E}^{2} \leq 0.49\right)$, which indicates that the Maintenance task may be very much dependent on the analyzed system itself.

Table 74. Comparing Maintenance I and Maintenance II - Main effects

\begin{tabular}{|c|c|c|c|c|c|c|}
\hline & \multicolumn{3}{|c|}{ Experiment I (two-way ANOVA) } & \multicolumn{3}{|l|}{ Experiment II (three-way ANOVA) } \\
\cline { 2 - 7 } & Effect & $\mathrm{p}$-value & $\mathrm{E}^{2}$ & Effect & $\mathrm{p}$-value & $\mathrm{E}^{2}$ \\
\hline Laboratory 2 & $\begin{array}{c}\text { System } \\
\text { (ANOVA with Method) }\end{array}$ & 0.004 & 0.26 & System & $<0.0001$ & 0.39 \\
\hline & $\begin{array}{c}\text { System } \\
\text { (ANOVA with Method) }\end{array}$ & $<0.0001$ & 0.47 & System & $<0.0001$ & 0.49 \\
\cline { 2 - 7 } & $\begin{array}{c}\text { Method } \\
\text { (ANOVA with System) }\end{array}$ & 0.013 & 0.11 & Method & 0.023 & 0.04 \\
\cline { 2 - 7 } & $\begin{array}{c}\text { Ability } \\
\text { (ANOVA with Method) }\end{array}$ & 0.006 & 0.22 & & & \\
\cline { 2 - 8 } & $\begin{array}{c}\text { Method } \\
\text { (ANOVA Ability) }\end{array}$ & 0.029 & 0.13 & & & \\
\hline
\end{tabular}

\subsubsection{Interaction effects}

There are no significant interaction effects in Experiment I. Significant interaction effects between Method \& Ability and Method \& System are visible in M-2-II, but not in M-4-II. The presence and absence of these interaction effects are difficult to interpret. The interactions between Method and other factors need further investigation in future experiments. 


\subsection{Discussion of results}

This section summarizes and discusses the analysis of the results for both experiment trials. As Method is the factor of interest in this thesis, only the significant effects relating to Method are discussed below.

Table 75 summarizes the effects of using OCL. The rows list all the tasks performed in each experiment trial. There are two attempts for each task. The results of both attempts analysis and each attempt (per Laboratory) analysis are provided. The columns Mean $(O C L)$ and Mean(no_OCL) present the mean values of the subjects' performance using and not using OCL, respectively. The p-value columns show whether using OCL has a significant positive effect for a particular task. P-values $\leq 0.05$ indicate existence of significant positive effects.

It is observed that the percentages (scores) of correctly detected defects and correctly answered questions are rather low (Defect Detection: $<15 \%$; Comprehension: $<60 \%$; Maintenance: $<50 \%$ ). The low performance may be due to a lack of experience with the tasks or with their inherent complexity. Even after having gone through thorough UML analysis and design training, fourth year engineering students may not be comfortable with handling large system analysis models and documents of such complexity. The course, in the context of which this experiment took place, provides their first opportunity to do so. It is however interesting to note that the scores are consistent with the 
experimenters' subjective evaluation of the difficulty level of the tasks: Comprehension being easier, Defect Detection, and Maintenance being more difficult.

Table 75. Effect of using OCL

\begin{tabular}{|c|c|c|c|c|c|c|c|}
\hline & & \multicolumn{3}{|c|}{ Both attempts } & \multicolumn{3}{|c|}{ Each attempt } \\
\hline & Task & $\begin{array}{l}\text { Mean } \\
(\mathrm{OCL})\end{array}$ & $\begin{array}{c}\text { Mean } \\
\text { (no_OCL) }\end{array}$ & $\mathrm{p}$-value & $\begin{array}{l}\text { Mean } \\
(\mathrm{OCL})\end{array}$ & $\begin{array}{c}\text { Mean } \\
\text { (no_OCL) }\end{array}$ & $\mathrm{p}$-value \\
\hline \multirow{6}{*}{ 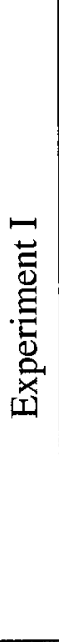 } & $\begin{array}{l}\text { Defect Detection } \\
\left(1^{\text {st }} \text { attempt: D-1-I }\right)\end{array}$ & \multirow{2}{*}{$11.67 \%$} & \multirow{2}{*}{$10.28 \%$} & \multirow{2}{*}{0.302} & $7.3 \%$ & $7.1 \%$ & 0.47 \\
\hline & $\begin{array}{l}\text { Defect Detection } \\
\left(2^{\text {nd }} \text { attempt: D-3-I }\right)\end{array}$ & & & & $\begin{array}{c}14.87 \\
\% \\
\end{array}$ & $14.23 \%$ & 0.44 \\
\hline & $\begin{array}{c}\text { Comprehension } \\
\left(1^{\text {st }} \text { attempt: C-2-I }\right)\end{array}$ & \multirow{2}{*}{$49.29 \%$} & \multirow{2}{*}{$42.86 \%$} & \multirow{2}{*}{0.028} & $41.7 \%$ & $45.9 \%$ & 0.85 \\
\hline & $\begin{array}{c}\text { Comprehension } \\
\left(2^{\text {nd }} \text { attempt: } \text { C-4-I }\right)\end{array}$ & & & & $57.5 \%$ & $39.65 \%$ & 0.002 \\
\hline & $\begin{array}{c}\text { Maintenance } \\
\left(1^{\text {st }} \text { attempt: M-2-I }\right)\end{array}$ & \multirow{2}{*}{$36.78 \%$} & \multirow{2}{*}{$31.76 \%$} & \multirow{2}{*}{0.159} & $28 \%$ & $32 \%$ & 0.8 \\
\hline & $\begin{array}{c}\text { Maintenance } \\
\left(2^{\text {nd }} \text { attempt: } M-4-I\right)\end{array}$ & & & & $46 \%$ & $31 \%$ & 0.02 \\
\hline \multirow{6}{*}{ 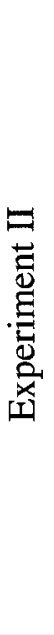 } & $\begin{array}{l}\text { Defect Detection } \\
\left.\text { (1 } 1^{\text {st }} \text { attempt: D-1-II }\right)\end{array}$ & \multirow{2}{*}{$9.48 \%$} & \multirow{2}{*}{$7.76 \%$} & \multirow{2}{*}{0.05} & $7.5 \%$ & $8.13 \%$ & 0.66 \\
\hline & $\begin{array}{c}\text { Defect Detection } \\
\left(2^{\text {nd }} \text { attempt: D-3-II) }\right.\end{array}$ & & & & $\begin{array}{c}11.87 \\
\%\end{array}$ & $7 \%$ & 0.003 \\
\hline & $\begin{array}{c}\text { Comprehension } \\
\left(1^{\text {st }} \text { attempt: C-2-II }\right)\end{array}$ & \multirow{2}{*}{$49.87 \%$} & \multirow{2}{*}{$42.36 \%$} & \multirow{2}{*}{0.0001} & $47 \%$ & $41 \%$ & 0.02 \\
\hline & $\begin{array}{c}\text { Comprehension } \\
\left(2^{\text {nd }} \text { attempt: } \mathrm{C}-4-\mathrm{II}\right)\end{array}$ & & & & $51 \%$ & $44 \%$ & 0.01 \\
\hline & $\begin{array}{c}\text { Maintenance } \\
\left(1^{\text {st }} \text { attempt: } \mathrm{M}-2-\mathrm{II}\right)\end{array}$ & \multirow{2}{*}{$41.37 \%$} & \multirow{2}{*}{$33.25 \%$} & \multirow{2}{*}{0.03} & $40 \%$ & $31 \%$ & 0.003 \\
\hline & $\begin{array}{c}\text { Maintenance } \\
\left(2^{\text {nd }} \text { attempt:M-4-II }\right)\end{array}$ & & & & $41 \%$ & $35 \%$ & 0.04 \\
\hline
\end{tabular}

The results show that, in Experiment I, when considering data from both attempts at the tasks together, using OCL has a significant positive effect for the Comprehension task only, but not for the Defect Detection or the Maintenance tasks. When considering data 
from each attempt, however, using OCL has positive effects for both Comprehension and Maintenance tasks in the second attempt, but not the first one. Although using OCL does not have any significant positive effect on the Defect Detection task, it has significantly different effects (interaction) on the high ability subjects and the low ability subjects in the second attempt as further discussed below.

On the other hand, in Experiment II, using OCL has significant positive effects for all the three tasks, whether taking all observations into account, or considering data from each attempt at the tasks, except the first attempt at the Defect Detection task. Further analysis shows that in the second attempt at the Defect Detection task, using OCL also has significantly different effects on the high ability subjects and the low ability subjects as further discussed below.

Therefore, there is strong evidence to suggest that using OCL results in significant benefits in terms of comprehension and maintenance. The effect for defect detection is more complex, as it depends on system characteristics, subjects' ability, and possibly other factors (further discussed below). Recall that the subjects of Experiment II received a more thorough OCL training than those of Experiment I. Therefore, in Experiment I, the subjects might have to make an additional effort to mature their OCL knowledge during the time period that the experiment trial was performed. This may explain why Method had significant main effects only for the last two tasks in Experiment I, and for most of the tasks in Experiment $\Pi$. It indicates that the subjects can take advantage of using OCL only after a substantial training in using OCL and UML. 
Table 75 also shows that the subjects' performance improvements from the first attempt to the second exist for some tasks, as illustrated in Figure 36. In Experiment I, there is likely a strong learning effect, as performance improvements are observed for most tasks, regardless of the order in which OCL is used. The learning effect confounds with the effect of OCL. In Experiment II, the learning effect seems to be weaker: performance improvement is only observed for the tasks when OCL is used in the second attempt, but not in the first attempt (N_O), which is consistent with what is observed in Experiment I; on the other hand, performance deterioration is observed when OCL is used in the reverse order $\left(\mathrm{O} \_\mathrm{N}\right)$, which is different from what is observed in Experiment $\mathrm{I}$.

\section{Figure 36. Illustration of learning effect}

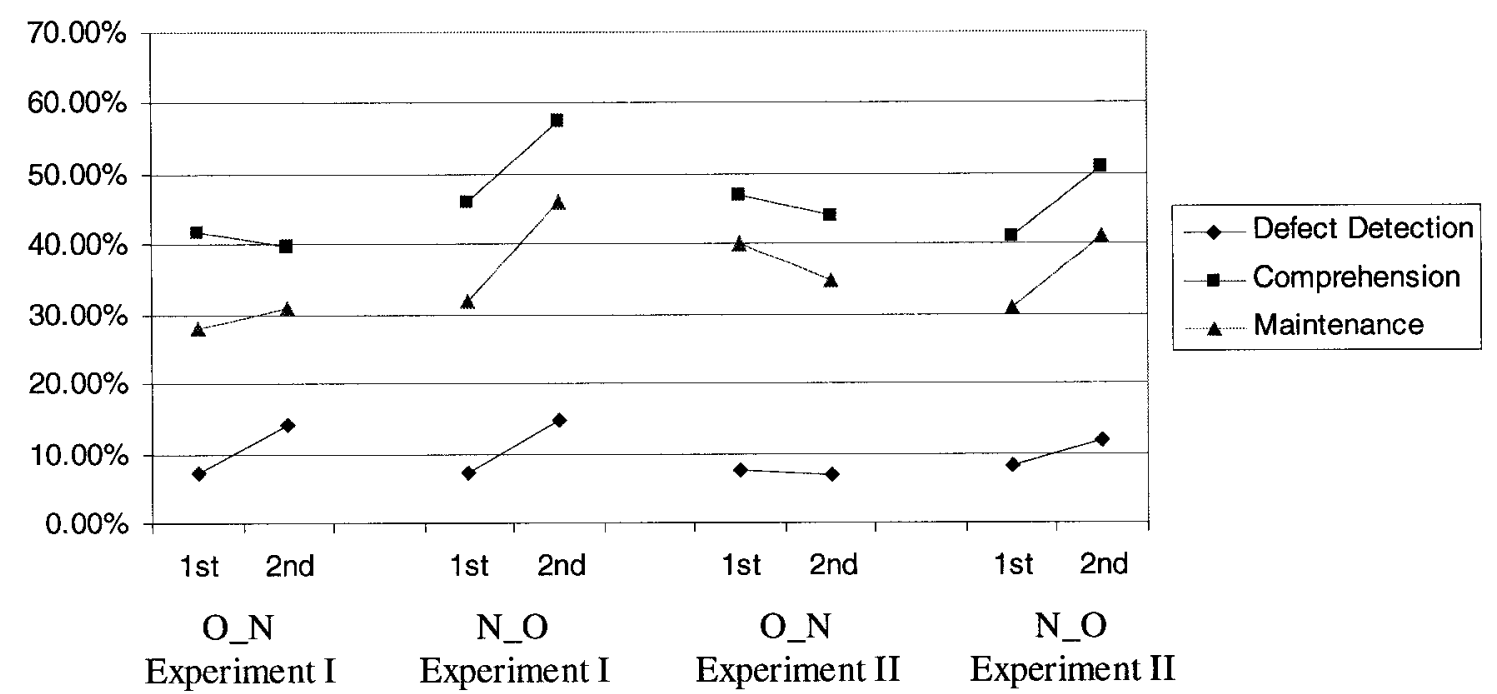

Statistics also show that the only significant performance improvement is experienced by those subjects who use OCL in the (N_O) order as shown in Table 76. This strongly 
indicates that the performance improvement is probably due to the presence of OCL expressions.

Table 76. Significant improvement by the subjects

\begin{tabular}{|l|l|l|}
\hline & Task & $\begin{array}{l}\text { Subjects with significant performance } \\
\text { improvement from } 1^{\text {st }} \text { attempt to } 2^{\text {nd }} \text { attempt }\end{array}$ \\
\hline \multirow{4}{*}{ Experiment I } & Defect Detection & Low ability, No_OCL first, then OCL (Table 18) \\
\cline { 2 - 3 } & Comprehension & High ability, No_OCL first, then OCL (Table 38) \\
\cline { 2 - 3 } & Maintenance & (None) \\
\hline \multirow{3}{*}{ Experiment II } & Defect Detection & High ability, No_OCL first, then OCL (Table 28) \\
\cline { 2 - 3 } & Comprehension & $\begin{array}{l}\text { High and low ability, No_OCL first, then OCL } \\
\text { (Table 48) }\end{array}$ \\
\cline { 2 - 3 } & Maintenance & Low ability, No_OCL first, then OCL (Table 67) \\
\hline
\end{tabular}

Table 76 also shows that using OCL seems to benefit the high ability subjects in some instances, and the low ability subjects in others instances. It indicates interactions between using OCL and subjects' modeling ability, which can only be investigated via the ANOVA analysis, as discussed below.

Table 77 summarizes the results of the ANOVA results for the three factors: Method, Ability, and System, for each laboratory of each experiment trial. The rows present all the main and interaction effects. The columns present all the tasks performed. For each task, if an effect is significant, an " $\mathrm{X}$ " is marked in the corresponding cell.

Method has significant main effects in C-4-I, M-4-I, D-3-II, C-2-II, C-4-II, M-2-II, and M-4-II. This is consistent with the results presented above. 
Table 77 Summary of the significant effects

\begin{tabular}{|c|c|c|c|c|c|c|}
\hline \multicolumn{7}{|c|}{ Experiment I } \\
\hline & D-1-I & D-3-I & $\mathrm{C}-2-\mathrm{I}$ & $\mathrm{C}-4-\mathrm{I}$ & $\mathrm{M}-2-\mathrm{I}$ & M-4-I \\
\hline Method & & & & $\mathrm{X}$ & & $\mathrm{X}$ \\
\hline Ability & $X$ & $\mathrm{X}$ & & $X$ & & $X$ \\
\hline System & & $\mathrm{X}$ & & $\mathrm{X}$ & $\mathrm{X}$ & $\mathrm{X}$ \\
\hline Method \& Ability & & $\mathrm{X}$ & & & & \\
\hline Method \& System & & & & & & \\
\hline \multicolumn{7}{|c|}{ Experiment II } \\
\hline & D-1-II & D-3-II & $\mathrm{C}-2-\mathrm{II}$ & C-4-II & M-2-II & M-4-II \\
\hline Method & & $\mathrm{X}$ & $\mathrm{X}$ & $\mathrm{X}$ & $X$ & $\mathrm{X}$ \\
\hline Ability & $\mathrm{X}$ & $\mathrm{X}$ & & $\mathrm{X}$ & & \\
\hline System & $\mathrm{X}$ & & & $\mathrm{X}$ & $\mathrm{X}$ & $X$ \\
\hline Method \& Ability & & $\mathrm{X}$ & & & $\mathrm{X}$ & \\
\hline Method \& System & & $\mathrm{X}$ & & & $\mathrm{X}$ & \\
\hline Ability \& System & & & & & & \\
\hline Method, Ability \& System & & & & & & \\
\hline
\end{tabular}

Method has significant interaction effects with Ability in D-3-I, D-3-II, and M-2-II. There are inconsistencies in the interactions between Method and Ability for Defect Detection that cannot be explained easily: using OCL hinders the high ability subjects in D-3-I, but helps them in D-3-II; while OCL helps the low ability subjects in D-3-I, but does not have any effect in D-3-II. However, the high ability subjects perceived OCL to be useful more than the low ability subjects in both D-3-I and D-3-II. A possible interpretation is that when the subjects are not familiar with using OCL, the high ability subjects do not need OCL to find defects, as their insight and intuition with respect to UML system analysis is sufficient for them to detect defects. Reading and analyzing OCL expressions therefore results in a time overhead without real benefit to them. On the other hand, the low ability subjects may use OCL in a mechanical way to detect inconsistencies in UML 
system analysis documents. Once more experience is gained in using OCL and UML system analysis, the high ability subjects eventually benefit from using OCL, while the low ability subjects do not need to rely on OCL mechanically to perform the tasks any more. Whether or not the low ability subjects would also benefit from using OCL after more training, needs to be further investigated in future experiments.

In M-2-II, using OCL seems to have a much greater positive effect on the low ability subjects than on the high ability subjects $(\operatorname{Diff}($ High $)=7 \%, \operatorname{Diff}($ Low $)=13 \%)$. This may be because the high ability subjects have a better understanding of UML system analysis than the low ability subjects. Thus the high ability subjects may obtain less benefit from using OCL than the low ability subjects for the Maintenance task. However, this interaction between Method and Ability is not visible in M-4-I. It is possible that the learning effect with respect to UML system analysis and the Maintenance task help the low ability subjects catch up with the high ability subjects during $\mathrm{M}-2-\mathrm{II}$, and resulting in a better performance in M-4-II. In turn, the positive effects of using OCL are similar for the high ability subjects as for the low ability subjects. The effect of the interaction, between Method and Ability, on different software engineering activities, such as detecting defects and maintenance, requires more investigation in future experiments.

Method also has significant interaction effects with System in D-3-II and M-2-II. In D-3II, using OCL results in a larger performance difference between the OCL subjects and the no_OCL subjects for the Video Store system than for the Cab Distribution system $(\operatorname{Diff}(\mathrm{VS})=10 \%, \operatorname{Diff}(\mathrm{CD})=0.6 \%)$. This suggests that with increased OCL knowledge, 
using OCL may have a larger impact on Defect Detection for more complex system models.

On the other hand, in M-2-II, using OCL results in a larger performance difference between the OCL subjects and the no_OCL subjects for the Cab Distribution system than for the Video Store system $(\operatorname{Diff}(C D)=17 \%, \operatorname{Diff}(V S)=3.9 \%)$. This suggests that using OCL may have larger impact on Maintenance for simpler system models. However, this interaction is not visible in M-4-II. The interaction effect between Method and System on different software engineering activities, such as detecting defects and maintenance, needs to be investigated further in future experiments.

The results of this study show that OCL, as a constraint language complementary to UML, is useful in helping understand system models, facilitate change and detect defects in such models. However, the interaction of subjects' modeling ability and system complexity with using OCL needs to be further studied and understood. Furthermore, the benefits of using OCL may only be observed if subjects have sufficient training and experience in UML and OCL. 


\section{CHAPTER 5 CONCLUSIONS}

This thesis investigates an important methodological aspect of the use of the Unified Modeling Language (UML) [2] for Object-Oriented Analysis and Design: Whether or not, the Object Constraint Language (OCL) [18], which is part of the UML standard, should be used to augment the precision of system model specifications. In the specific context of UML, this question is very much akin to the ongoing debate about the required level of formalism and precision in software development $[16,17]$. There was no known experiment conducted prior to the attempt presented in this thesis to investigate this question.

Two trials of the experiment (Experiment I and Experiment II) were performed with $4^{\text {th }}$ year Software/Computer Engineering students who received substantial training in UML and OCL. This experiment looked into the impact of using OCL on three software engineering activities: (1) answer questions about the underlying logic of modeled systems, (2) perform an impact analysis of changes to UML models, and (3) detect defects through model inspections. It was important to ensure that the controlled experiment would yield valid results, and that the trends observed were due to the use of OCL and not other extraneous factors. Careful experiment design was undertaken so as to prevent confounding effects, such as effects related to a widely varying ability of students to use UML system models and the different characteristics of the UML system models. 
In order to yield conservative results, all three tasks are designed such that questions can be answered, and defects can be detected with or without OCL. Further, not only the effect of using OCL was examined, but also its interactions with students' modeling ability and systems used to understand the relationship between using OCL and other factors.

As discussed in greater detail in Section 4.4, results show that using OCL has a positive impact on the effectiveness of performing software engineering activities, such as Comprehension and Maintenance. That impact is both statistically and practically significant. The effect of using OCL can be very large: e.g., differences between subjects using OCL and not using OCL could vary between $0 \%$ and $40 \%$ for Comprehension, and between $10 \%$ and $89 \%$ for Maintenance. The range of differences is large, as it also depends on other factors, such as students' modeling ability and system used. In practice, it is important to correctly understand and maintain a system during early analysis/design stages, as implementation errors caused by misunderstanding the analysis would be very expensive to correct at later stages. Therefore, the above differences have practical importance. This then suggests that the cost-benefit of using OCL is worth investigating further in industrial settings, especially considering that OCL is also potentially beneficial for other reasons not investigated here, e.g., its impact on the quality of the resulting application implementations. The difference for Defect Detection varies between $-42 \%$ and $313 \%$, which indicates that the effectiveness for Defect Detection may be lower ($42 \%$ ) or much higher (313\%) when OCL is used. Therefore, the impact of OCL for this 
task strongly depends on other factors and it is important that this issue be investigated in the future. Furthermore, the effects of using OCL take different forms in the two experiment trials. In Experiment I, the positive effect of using OCL was only observed the second time the students performed the tasks. This was in fact to be expected as the students' UML and OCL training did not include much experience with documents of the level of complexity exhibited in the UML models used in the experiment. The students also had little experience with respect to the specifics of the tasks performed.

In Experiment II, the students received more prior training in OCL, UML and the tasks, and also had an additional week to mature their OCL and UML knowledge between laboratories than their fellow students of Experiment I. In turn, more positive effects of using OCL were observed in Experiment $I I$ than in Experiment I. This suggests that substantial prior training is required to take the advantage of using OCL.

Furthermore, significant interactions between using OCL and the students' modeling ability were observed for Defect Detection in Experiment I, and for Defect Detection and Maintenance in Experiment II. Using OCL sometimes favors high ability students, and sometimes low ability students. Moreover, significant interactions between using OCL and different systems were also observed for Defect Detection and Maintenance in Experiment II. Using OCL sometimes favors a simpler system analysis, and sometimes a more complex system analysis. More investigation is required to study these interactions in future experiments. 
In conclusion, the results of the experiment show that using OCL has the potential to significantly improve engineers' ability to inspect and modify UML system analysis documents, but significant prior training and experience are required. This is not surprising as it is often the case that new methodologies or technologies take substantial time to assimilate before they start paying off [15]. From a practical perspective, this means that it is unlikely that the engineers will benefit from using OCL in UML models, unless they are properly trained. Based on the experimenters' observations, this requires that the level of training be much more substantial than what is typically observed across the software industry. This is, however, likely to become increasingly easier as new software engineering graduates, who have received substantial UML and OCL training, permeate software development organizations and as the UML system analysis standard attains a firmer penetration. 


\section{CHAPTER 6 FUTURE WORK}

This chapter describes possible future work to further investigate the impact of using OCL. Section 6.1 details the issues that could not be explained by the available experimental data. Section 6.2 provides suggestions of how these issues may be addressed in future experiments. Section 6.3 concerns other aspects of using OCL that may be of interest.

\subsection{Issues encountered in the experiment}

There are a few issues which could be investigated further, as the existing experimental data does not provide support for clear explanations.

Regarding the Defect Detection task, it is difficult to explain why OCL seemed to hinder high ability students in detecting defects in Experiment I, while help them in Experiment II. Regarding the Maintenance task, it is also difficult to interpret why significant interactions between using OCL and students' modeling ability, using OCL and the systems used, were visible only during the first attempt at the Maintenance task in Experiment II, but not during the second attempt.

Furthermore, with respect to the Maintenance task, it was observed that students' modeling ability had a significant effect in Experiment I, but not in Experiment II. It 
could not be determined whether a trend exists: the more OCL and UML knowledge the subjects gain, the less of an impact students' modelling ability has. It was also observed that the survey responses were not consistent with, or did not support the laboratory observations.

Lastly, the performance improvement from the first attempt at a task to the second was observed during the experiment, but could not always be precisely explained: Was the performance improvement due to an improved understanding of UML system analysis or more related to gained familiarity with the tasks to be performed? Was it perhaps due to an increased level of comfort with using OCL? Although tentative interpretation was given, further statistical evidence is desirable.

\subsection{Considerations for future experiments}

Future experiments could perhaps address the following aspects: subjects' experience with real-scale UML system analysis and post-lab interviews.

In addition to a thorough OCL and UML system analysis training, the subjects should ideally have prior experience in performing UML system analysis and using OCL on real-scale systems. By doing so, learning effect may be attenuated, so that effects of using OCL stand out from the data, and therefore, one may be able to provide better interpretations for the abovementioned issues. However, in an academic environment, this may not be achievable. An alternative is to have one more distinct experimental 
group of subjects to work on UML system analysis documents without (or with) OCL in all laboratories, as a reference group. This way, the performance improvement over time for each one of the existing four groups (formed in this experiment) can be analyzed in conjunction with the learning effect that may be observed for the reference group.

Moreover, a post-lab interview is also desirable as a supplement to the post-lab survey. Post-lab interviews would allow experimenters to ask more questions, which may be raised during the execution of the experiment, or to clarify survey responses from the subjects. For example, is there any difference between the perception of the usefulness of OCL for the first and the second attempt at a task? Do the subjects find it easier to perform the task during the second attempt than during the first attempt? Responses to these questions can be used to better understand learning effect.

\subsection{Other aspects of interest}

Although the presence of OCL in UML system analysis documents has positive effects on software engineering activities, as reported in this thesis, can the benefit justify the cost of documenting OCL expressions? Using OCL is only practical, if the gained benefit is larger than the cost. It is possible to study the cost of using OCL by comparing the effort used in creating a UML system analysis document with and without OCL. There are, however, other considerations which need to be taken into account, such as how to 
measure cost, the quality of implementation, the correctness and completeness of UML models, etc.

There are other areas which may be interesting. This experiment uses typical UML analysis documentation. If much more detailed descriptions are provided in natural language, will OCL's formality bring any extra benefit? Which one is more costeffective: detailed description in natural language or in OCL? Further, objects constraints can be described in OCL in many ways. The style of OCL documentation could have an impact on the effectiveness of using OCL. Furthermore, there may also exist tools to help generate OCL expressions, or to check analysis consistency automatically, which may or may not affect the experiment results. 


\section{References}

[1] E. Arisholm and D. Sjøberg, A Controlled Experiment with Professionals to Evaluate the Effect of a Delegated versus Centralized Control Style on the Maintainability of Object-Oriented Software, Simula Research Laboratory, Technical Report 2003-6, http://www.simula.no/, 2003.

[2] G. Booch, J. Rumbaugh and I. Jacobson, The Unified Modeling Language User Guide, Addison Wesley, 1999.

[3] L. C. Briand, Y. Labiche and H. Sun, Investigating the Use of Analysis Contracts to Improve the Testability of Object-Oriented Code, Software - Practice and Experience, vol. 33 (7), pp. 637-672, Wiley, 2003.

[4] B. Bruegge and A. H. Dutoit, Object-Oriented Software Engineering - Conquering Complex and Chalenging Systems, Prentice Hall, 2000.

[5] S. Cook, A. Kleppe, R. Mitchell, B. Rumpe, J. Warmer and A. Wills, The Amsterdam Manifesto on OCL, in T. Clark and J. Warmer, Eds., Object Modeling with the OCL, The Rationale behind the Object Constraint Language, vol. 2263, Lecture Notes In Computer Science (LNCS), Springer Verlag, 2002, pp. 115-149.

[6] J. L. Devore and N. Farnum, Applied Statistics for Engineers and Scientists, Duxbury, 1999.

[7] H. Gomaa, Designing Concurrent, Distributed, and Real-Time Applications with UML, Object Technology, Addison Wesley, 2000.

[8] P. Henderson, Mathematical Reasoning in Software Engineering Education, Communications of the ACM, vol. 46 (9), pp. 45-50, 2003.

[9] G. Iversen and H. Norpoth, Analysis of Variance, Sage Publications, second Ed., 1987.

[10] A. Kleppe, J. Warmer and W. Bast, MDA Explained - The Model Driven Architecture: Practice and Promise, Addison-Wesley, 2003.

[11] C. Larman, Applying UML and Patterns - An Introduction to Object-Oriented Analysis and Design and the Unified Process, Prentice Hall, second Ed., 2002. 
[12] R. Mitchell and J. McKim, Design by Contract, by Example, Addison-Wesley, 2001.

[13] OMG, Object Constraint Language - OCL, Object Management Group V1.4, 2001.

[14] OMG, Unified Modeling Language (UML), Object Management Group V1.4, www.omg.org/technology/uml/, 2001.

[15] A. N. Oppenheim, Questionnaire Design, Interviewing and Attitude Measurement, Pinter Publishers, 1992.

[16] S. L. Pfleeger, Understanding and improving technology transfer in software engineering, Journal of Systems and Software, vol. 47 (2-3), pp. 111-124, 1999.

[17] S. L. Pfleeger and L. Hatton, Investigating the Influence of Formal Methods, Computer, vol. 30 (2), pp. 33-43, 1997.

[18] J. Warmer, A. Kleppe, The Object Constraint Language: Precise Modeling with $U M L$, Addison Wesley Longman, Inc, 1999

[19] A. E. K. Sobel and M. R. Clarkson, Formal Methods Application: An Empirical Tale of Software Development, IEEE Transactions of Software Engineering, vol. 28 (3), pp. 308-320, 2002.

[20] C. Wohlin, P. Runeson, M. Host, M. C. Ohlsson, B. Regnell and A. Wesslen, Experimentation in Software Engineering - An Introduction, Kluwer, 2000.

[21] Rand R. Wilcox, Statistics for the Social Sciences, Academic Press, 1996

[22] Cook TD, Campbell DT, Experimental and Quasi-Experimental Designs for Research, Boston MA, Boughton Mifflin Company, 1979

[23] Natalia Juristo and Ana M. Moreno, Basics of Software Engineering Experimentation, Kluwer Academic Publishers, 2001

[24] Bertrand Meyer, Object-Oriented Software Construction, Prentice-Hall, 1988

[25] Jerome L. Myers, Fundamentals of Experimental Design, Second Edition, Allyn and Bacon, Inc., Boston, 1972

[26] V. R. Basili, R. W. Selby and D. H. Hutchens, Experimentation in Software Engineering, IEEE Transactions on Software Engineering, 1986 
[27] Dr. Jim Robison-Cox, Mathematical sciences department, Montana state university, Two factor ANOVA - Analysis when sample sizes are not equal, URL: http://www.math.montana.edu/ jimrc/classes/stat412/inclass/p69.unequalSamplSiz es.pdf 


\section{Appendix A Seeded Defects}

\section{Table 78. Defects seeded in the Cab Distribution System}

Class Diagram (Entity)

1. Remove "Subset" association between Job and Cab

2. Change the cardinality of association from Cab to Driver from "0..1-0..1" to "0..1 - *"

3. Remove the association between Job and Customer

4. Swap cardinality of association between Job and Cab (dispatchedTo)

5. Exchange role names "bookedCab" \& "performedBy"

Data Dictionary

6. Cab: remove "Off" of cabState

7. Cab::assignCab(j:PrebookedJob): change the description from "... other prebooked jobs in 2 hours" to "... other immediate jobs in 2 hours"

8. Cab::dispatchCab(j:ImmediateJob): change the description from "... other prebooked jobs in 2 hours" to "... other immediate jobs in 2 hours"

9. Customer::getNotInvoicedJobs(): change from "active jobs" to "all jobs" in the description

10. Driver::performedJobs(): Set(Job): change the description from "Returns all the Jobs..." to "Returns all the immediate jobs and prebooked jobs..."

11. PrebookedJob::verifyPreJob(): change description and return-list from "... confirmed..." to " ... dispatched..."

12. PrebookedJob::cancel(): change to "... be done 20 hours before..."

13. JobManagerControl::CheckDispatch(): change from "prebooked" jobs to "all" jobs.

14. JobManagerControl::SendCab(): add "or a prebooked Job" to the description

15. RequestCabForm::bookCab(): add "or an immediate Job" to the description

Sequence Diagram (HandleImmediateJob)

16: Change 2.1.2 from "dispatchCab(j)" to "assignCab(j)"

17. Immediate job constructor called by sendCab(), change to called by requestCab()

18. Other operations called by send $\mathrm{Cab}($ ), changet o called by the constructor of immediate job

Sequence Diagram (LogPrebookedJob)

19. Swap 1.2.2 and 1.2.3

Sequence Diagram (DispatchPrebookedJob)

20. 1.2.8 add [ $\mathrm{v}=$ true] guard condition

21. Remove "[v=true]" in 1.2.2 


\section{Swap 1.2.2 and 1.2.3}

23. Remove 1.2.6, and make all operations called by 1.2 .6 to be called by the Immediate Job constructor

State Chart (Prebooked Job)

24. Remove the transition from "Pending" to itself

25. Remove guard condition of transition form "Pending" to "Cancelled"

State Chart (Cab)

26. Remove the transition from "Idle" to "Busy"

27. Swap the 2 transitions of cancelCab() from "Assigned"

28. Swap the transitions of "assignCab" from Assigned to self and "dispacthCab" from Assigned to Busy

29. Remove the guard condition of the transition from "Busy" to "Idle"

30. Remove the transition from "Busy" to "Assigned"

\section{Table 79. Defect seeded in the Video Store System}

\section{Class Diagram (Entity)}

1. Change the association from "0..1 $-0 . .1$ " to "* $-0 . .1$ " connecting Reservation and Copy

2. Remove association "Subset" between 2 associations connecting Reservation and Title

3. Change the association from "oldestpending" to "outstanding" connecting Reservation and Title

4. Remove role name "reservation" connecting Reservation and Title

5. Swap role names of "borrower" and "rentals" connecting Member and Rental

Data Dictionary

6. Copy::checkCopy(): switch copy status 1 and 2

7. Copy::getReservation(): change the description to "Returns all the pending reservations." from "Returns the reservation that held the current copy if any"

8. Copy::makeAvailable(): remove "or rental" from the description

9. Member::checkMember(): switch member status 1 and 2

10. Member::removeReservation(reserve: Researvation): change the description from "Removes a reservation..." to "Removes a pending reservation..."

11. Reservation:cancel(), change description to " ... pending reservation..." from "... current reservation", remove "or Outstanding" at the end

12. Reservation::copyAvailable(c:Copy): change the description from "... another reservation", to "...another rental"

13. Reservation:expire(), change description to ".. 60 hours.." from " ... 48 hours"

14. Reservation::fulfill(r:Rental): change the description from "Fulfills the current reservation,..." to "Fulfills a pending or outstanding reservation, ..." 
15. Title::changeOldestPending(): change the description from "Changes the oldest pending reservation" to "Change the outstanding reservation to the oldest reservation" Sequence Diagram (Rent Video)

16. Remove condition "...(s2=1...)" from $1.2 .11,1.2 .12,1.2 .13$

17. Change to "Rental()" calls $1.2 .12,1.2 .13$, rather than "rentVideo()"

18. Remove 1.2.5

Sequence Diagram (Reserve Video)

19. Remove 1.2 .5

20. Add "or $\mathrm{s} 2=1$ " into 1.2.8, 1.2.9, and 1.2.10

21. Change from Reservation() calls 1.2.8.1, 1.2.8.2 to ReservationControl calls State Chart (Copy)

22, 23. Change from "Pending" to "Outstanding" on the 2 transitions from OnHold state

24. Swap 2 return() transitions from Rented state

State Chart (Reservation)

25. Change from "oldestPending" to "reservation" on the transition from Pending to Outstanding

26, 27. Change from "Pending" to "Outstanding" on 2 transitions from Outstanding state to Expired state

28. Remove the transition from "Pending" to "Cancelled"

29. Change the action to "title.getOldestPending()" from \#Pending to \#Outstanding (correct action: "title.changeOldestPending()")

30. Remove a transition from "Outstanding" to "Cancelled" 


\section{Appendix B Questionnaires}

\section{B.1 Cab Distribution System}

\section{B.1.1 Comprehension questionnaire}

1. Describe the state invariant for state assigned of class Cab
A. The cab has a current driver, the cab is dispatched to a job, the cab does not have a current job
B. The cab has a current driver, the current driver's mode is on, the cab is dispatched to a job
C. The cab has a current driver, the current driver's mode is on, the cab is dispatched to a job, the cab does not have a current job
D. The cab has a current driver, the cab is dispatched to a job
E. Other answer:

2. After a job is completed, what is the Cab's state?
A. idle
B. assigned
C. busy
D. idle or assigned
E. assigned or busy
F. idle or assigned or busy
G. Other answer:

3. After a job is cancelled, what is the associated Cab's state?
A. idle
B. assigned
C. busy
D. idle or assigned
E. assigned or busy
F. idle or assigned or busy
G. Other answer: 
4. What kind of Cab can be booked for a prebooked job?
A. Any idle cabs
B. Any idle or assigned cabs
C. Any cabs, that are not off
D. Any idle or assigned cabs, with no other jobs within 2 hours after the job's pickup time
E. Other answer:

5. What kind of Cab can be dispatched to an immediate job?
A. Any idle cabs
B. Any idle or assigned cabs
C. Any cabs, that are not in state of off
D. Any idle or assigned cabs, with no other jobs within 2 hours after the job's pickup time
E. Other answer:

6. Describe the state invariant for state dispatched of class ImmediateJob.
A. The immediate job is performed by a cab. The cab's current job is the immediate job. The cab's state is assigned.
B. The immediate job is performed by a cab. The cab's current job is the immediate job. The cab's state is busy.
C. The immediate job is performed by a cab. The cab's current job is the immediate job. The cab's state is assigned or busy.
D. Other answer:

7. Which attributes of class Job are to be checked before a PrebookedJob is confirmed?
A. pickuploc, pickupTime, jobstate
B. pickuploc, deliveryloc, pickupTime
C. pickuploc, deliveryloc, pickupTime, jobstate
D. pickupTime
E. Other answer

8. After a cab has been sent to service a job by the system, which association(s) and / or attribute(s) is (are) changed?
A. currentJob, performedBy
B. currentJob
C. cabstate, currentJob
D. cabstate, currentJob, performedBy
E. Other answer

9. Following the previous question, which attribute(s) of currentJob is (are) changed
A. pickuploc
B. pickuploc, pickupTime
C. pickuploc, jobstate
D. pickuploc, pickupTime, jobstate 
E. Other answer

10. Each time a checkDispatch() is performed by the Calendar and Clock System, the iteration of messages in the DispatchPrebookedJob sequence diagram is executed:
A. once
B. for all prebooked jobs
C. for prebooked jobs whose pickupTime is 15 minutes from the current time
D. for prebooked jobs whose pickupTime is $15-20$ minutes from the current time
E. Other answer

11. Which operation(s) access(es) the financialStatus attribute?
A. Customer::getNotInvoiceJobs(), InvoiceAccountCustomer::printList()
B. Customer::getNotInvoiceJobs(), CustomerControl::printList(), InvoiceAccountCustomer::printList()
C. Customer::getNotInvoiceJobs(), CustomerControl::printList(), CustomerControl::chargeAccount(), InvoiceAccountCustomer::printList()
D. Other answer

12. Which class(es) is(are) to be involved in the Print Invoice Account Customer use case? (Note: no sequence diagram is available for that use case)
A. InvoiceAccountCustomer
B. Customer, Job, InvoiceAccountCustomer
C. Customer, CustomerControl, InvoiceAccountCustomer
D. Customer, CustomerControl, Job, Invoice AccountCustomer
E. Other answer

13. What characterizes the "closest cab", when an immediate job is being dispatched?
A. A cab with the shortest ETA
B. An idle cab with the shortest ETA
C. An idle or assigned cab with the shortest ETA
D. A cab, with the shortest ETA, which hasn't been booked for a job in the next 2 hours.
E. Other answer

14. What does use case "Print invoice account customer" print out?
A. All jobs
B. All completed jobs
C. All jobs, that have not been invoiced
D. All completed jobs, that have not been invoiced
E. Other answer

15. After a cab is booked for a job, the cab's state should be:
A. idle or assigned
B. idle or busy
C. assigned or busy 
D. idle, or assigned, or busy

E. Other answer

16. When a cab is in state assigned, which one of the following statements is correct?

A. The cab's currentJob must be empty. The cab's dispatchedTo must be empty.

B. Both the cab's currentJob and dispacthedTo must not be empty.

C. The cab's currentJob must not be empty. The currentJob's state should be "dispatched".

D. None of the above

17. Which one of the following statements is correct?

A. When a job is created, the pickup location may be empty.

B. When a job is created, the pickup time must not be empty.

C. An immediate job can be cancelled.

D. A prebooked job is completed when the driver picks up the customer.

E. None of the above

18. When a cab is booked, which one of the following statements is correct?

A. The pickuploc may be empty, the pickupTime may be empty. The state of the job is "pending".

B. The pickuploc must not be empty, the pickupTime may be empty. The state of the job is "confirmed".

C. The pickuploc may be empty, the pickupTime must not be empty. The state of the job is "pending".

D. The pickuploc must not be empty, the pickupTime must not be empty. The state of the job is "pending".

E. The pickuploc must not be empty, the pickupTime must not be empty. The state of the job is "confirmed".

F. None of the above

19. Which one of the following statements is correct?

A. The satellite sends all cab's location to the system.

B. The satellite sends the idle cab's location to the system only.

C. The satellite sends the idle and assigned cabs' locations to the system only.

D. The satellite sends the cabs that are not in the state of "off" only.

E. None of the above

20. Which one of the following statements is correct?

A. A customer can have more than 1 account.

B. Cab service fee will be charged to the customer's account, if the customer asks the driver to do so.

C. When a cab is dispatched, the type of the associated job must be an immediate job.

D. None of the above 


\section{B.1.2 Maintenance questionnaire}

1. A constant, additional charge must be automatically added when the Job begins or ends during night time (e.g. 9:00pm - 7:00am). The time may be changed by the system administrator.

a. Which classes are affected?

b. Which attribute(s) / association(s) should be added?

c. Which operation(s) must be changed? Describe the new behaviour of these operations? (Please provide a complete answer.)

2. A location is currently an attribute of type string. Suppose we change it to an instance of class Location: class Location has attributes for the street number, the building, the phone number, etc.

a. Which classes are affected by this change?

b. Which attributes must be changed/added/removed?

c. Which operations are affected by this removal? (indicate both class and operation names, i.e., className::operationName)

3. A maintainer erroneously removed the currentJob association.

a. Which classes are affected by this removal?

b. Which operations are affected by this removal? (indicate both class and operation names, i.e., className::operationName)

c. Do you think this association is necessary? Why? 
4. A maintainer erroneously removed the dispatchedTo association.

a. Which classes are affected by this removal?

b. Which operations are affected by this removal?

(indicate both class and operation names, i.e., className::operationName)

5. The system has to handle the scenario when a cab is picking up customers in the streets. What change(s) should be made to the system? Which class(e)s will be affected?

6. In the original system, a job cannot be assigned to a cab that has a prebooked job within 2 hours after the pickup time. This is to ensure that drivers have enough time to perform jobs. However, it was overlooked that there might be prebooked job 2 hours before the pickup time. To fix this problem, what change(s) should be made to the system? Which operation(s) will be affected? (indicate both class and operation names, i.e., className::operationName)

\section{B.2 Video Store System}

\section{B.2.1 Comprehension Questionnaire}

1. Which attributes and / or associations should be checked to verify that a video copy is reserved?

A. The attribute onsale is false. There is no currentRental linked to the copy. There is reservation linked to the copy.

B. The attribute onsale is false. There is no currentRental linked to the copy. There is reservation linked to the copy.. The reservation is outstanding.

C. The attribute onsale is false. There is no currentRental linked to the copy. There is reservation linked to the copy.. The reservation is pending. 
D. The attribute onsale is false. There is no currentRental linked to the copy.

E. Other answer:

2. Which attributes and / or associations should be checked to verify if a video copy is available for rent?

A. The attribute onsale is false. There is no currentRental linked to the copy.

B. The attribute onsale is false. There is no currentRental linked to the copy. There is no heldcopy linked to the copy.

C. The attribute onsale is false. There is no currentRental linked to the copy. There is no reservation linked to the copy.

D. The state of the copy is ForRent. The attribute onsale is false. There is no heldcopy linked to the copy.

E. Other answer:

3. Which attributes and / or associations should be checked to verify that a reservation is pending?

A. The attribute whenOutstanding is undefined.

B. The attribute whenOutstanding is undefined. The attribute whenFulfilled is undefined.

C. The attribute whenOutstanding is undefined. The attribute whenFulfilled is undefined. The attribute whenCancelled is undefined.

D. The attribute whenOutstanding is undefined. The attribute whenFulfilled is undefined. The attribute whenCancelled is undefined. The attribute whenExpired is undefined. There is no heldcopy linked to the video copy.

E. There is a title associated with the reservation. The attribute whenOutstanding is undefined. The attribute attribute whenFulfilled is undefined. The attribute whenCancelled is undefined. The attribute whenExpired is undefined.

F. Other answer:

\section{Which attributes and / or associations should be checked to verify that a} reservation is outstanding?

A. The attribute whenOutstanding is set. The attribute whenFulfilled is undefined. The attribute whenCancelled is undefined. The attribute whenExpired is undefined.

B. The attribute whenOutstanding is set. The attribute whenFulfilled is undefined. The attribute whenCancelled is undefined. The attribute whenExpired is undefined. There is one heldcopy linked to the video copy.

C. The attribute whenOutstanding is set. The attribute whenFulfilled is undefined. The attribute whenCancelled is undefined. The attribute whenExpired is undefined. There is no heldcopy linked to the video copy. 

D. The attribute whenOutstanding is set. attribute The whenFulfilled is undefined. The attribute whenCancelled is undefined. The attribute whenExpired is undefined. There are one or more heldcopy linked to the video copy.
E. Other answer:

\section{Which attributes and / or associations should be checked to verify that a reservation has expired?}
A. The attribute whenExpired is less than the current time.
B. The attribute whenExpired is bigger than the current time.
C. The attribute whenExpired is set.
D. There is a link between the Title object and the Copy object.
E. Other answer:

6. Which attribute(s) in which class(es) are modified (and how are they modified) by a call to setForSale on a Title? (
A. The attribute onSale of Copy class is set to "True".
B. The Title state is set to ForSale.
C. The Title state is set to ForSale. The attribute salePrice is set to an amount.
D. All copies are transited to the state of ForSale. The attribute salePrice is set to an amount.
E. Other answer:

7. Suppose all the copies for Title $t$ are on hold. Can a member still rent a copy of $t$ ?
A. No, a member cannot rent a copy of $t$, until a reservation expires or is cancelled.
B. Yes, if the member has a reservation for $t$.
C. Yes, if the member has the oldest pending reservation for $t$.
D. Yes, if the member has an outstanding reservation for $t$.
E. Other answer:

\section{Which attributes and / or associations are affected by operation return() of Copy?}
A. The attribute whenCompleted will contain the current date and time.
B. The attribute whenCompleted will contain the current date and time. The attribute payment will contain the payment value.
C. The attribute whenCompleted will contain the current date and time. The attribute payment will contain the payment value. If the copy is overdue, the attribute payment value will be incremented by a fine.
D. The attribute whenCompleted will contain the current date and time. The attribute payment will contain the payment value. If the copy is overdue,


the attribute payment value will be incremented by a fine. The oldestPending reservation will become outstanding.

E. Other answer:

\section{Which attributes and / or associations must be checked in order for a Member $m$ to perform a reservation on Title $t$ ?}
A. $m$ must be active. $m$ must have reservation privilege. All copies must be rented or on hold.
B. $m$ must be active. $m$ must have reservation privilege. All copies must be rented.
C. $m$ must be active. $m$ must have reservation privilege. There must not exists a copy of the title that is for rent or on hold.
D. $m$ must be active. $m$ must have reservation privilege. There must exist a copy of the title that is for rent or on hold.
E. Other answer:

10. When a reservation, say $r$, is cancelled, are other reservations on that title affected?
A. No, no other instances of the class reservation will be affected.
B. Yes, if $r$ was outstanding, the oldest pending reservation becomes outstanding.
C. Yes, the oldest pending reservation becomes fulfilled.
D. Yes, if $r$ was the oldest pending reservation, the second oldest pending reservation becomes the oldest pending.
E. Yes, if $r$ was outstanding, the oldest pending reservation becomes outstanding. And, if $r$ was the oldest pending reservation, the second oldest pending reservation becomes the oldest pending.
F. Other answer:

\section{Which one of the following statements indicates that a member has valid status?}
A. The member's card is active.
B. The member has borrowing privilege.
C. The member's card is active and has borrowing privilege.
D. None of the above.

12. The following attributes and / or associations should be checked before a reservation can be cancelled:
A. The reservation exists. The state of the reservation is Pending.
B. The reservation exists. The state of the reservation is Outstanding.
C. The reservation exists. The state of the reservation is Pending or Outstanding. 
D. The reservation exists. The state of the reservation is Pending and the reservation should be the oldest pending, or the state of the reservation is Outstanding.

E. None of the above

13. What characterizes a video that is ready for reservation?

A. There exists at least one copy not on sale or sold, and no copy is for rent.

B. No copy is on sale or sold, and no copy is for rent.

C. There exists at least one copy not for rent.

D. No copy is on sale or sold, and there exists at least one copy not for rent.

E. Other answer:

14. The following attributes and / or associations should be changed when a video is reserved (reservation $\mathbf{r}$ is created):

A. Link the reservation to the member. Link the reservation to the video title. Set r's state to Pending. Set r's attribute whenMade to current time.

B. Link the reservation to the member. Link the reservation to the video title. Set r's state to Outstanding. Set r's attribute whenMade to current time.

C. Link the reservation to the member. Link the reservation to the video title. Set r's state to Pending. Link the reservation to a video copy. Set r's attribute whenMade to current time

D. None of the above

15. Which one of the following statements is correct?

A. Both outstanding and pending reservation can be expired.

B. An outstanding reservation can be expired, but not a pending reservation.

C. A pending reservation can be expired, but not an outstanding reservation.

D. Neither outstanding, nor pending reservation can be expired.

E. None of the above

16. What is the copy's state, after it is made available?
A. OnHold
B. ForRent
C. OnHold or ForRent
D. Other answer:

17. What kind of reservation can be removed from a member's reservation list by operation Member::removeReservation()?
A. Any type of reservations
B. Any type, but Outstanding
C. Any type, but Pending
D. Any type, but Outstanding or Pending
E. Other answer:

18. When a reservation is created, which one of the following statements is correct?

A. If there was no oldestPending reservation, it becomes oldestPending. 
B. If there was no outstanding reservation, it becomes outstanding

C. If there was no oldestPending, it becomes the oldestPending. If there was no Outstanding reservation, it becomes outstanding.

D. None of the above.

19. Which association(s) is (are) affected, when fulfil(r:Rental) is called on a reservation?
A. There is no link between the reservation and a video copy.
B. There is no link between the reservation and a video copy. The video copy does not have a reservation linked to it.
C. There is no link between the reservation and a video copy. There is a link between the rental and the reservation.
D. There is no link between the reservation and a video copy. There is a link between the rental and the reservation. The oldestPending becomes Outstanding
E. Other answer:

\section{What needs to be checked when class Title is in the state of \#ForSale?}
A. All copies of the title should be in the state of ForSale.
B. All copies of the title should be in the state of ForSale or Sold.
C. All copies of the title should be in the state of ForSale or Sold, with at least one copy in the state of ForSale.
D. Other answers:

\section{B.2.2 Maintenance Questionnaire}

1. Please use your own word to describe what should happen when a reservation is cancelled. (Do not copy / paste from the document.)

2. The video store company decides to modify Member's privileges, in order to allow/disallow the members to reserve and rent high-rated videos. It implies an additional Boolean attribute in class Title, in order to distinguish high-rated videos.

a. Which class(es) must be modified? 
b. Which modifications (addition, change of attributes, operations, and/or operations' contracts) must be made in this (these) class(es)?

c. Which message labels must be changed in the RentVideo sequence diagram? How?

d. Which message labels must be changed in the ReserveVideo sequence diagram? How?

3. The video store company decides to limit the maximum number of unfulfilled reservations that a Member can perform in a day. Each member may have a different limit.

a. Which class(es) must be modified?

b. Which modifications (addition, change of attributes, operations, and/or operations' contracts) must be made in this (these) class(es)?

4. An absent-minded maintainer accidentally removed the oldestPending association between Reservation and Title.

a. Which operations of which entity or control classes will be affected by this error?

5. It is required to add a new function to the system. When there are more than a given number of reservations on a given video at one time, the administrator should be notified to purchase more copies of this video.

a. Which class should be modified?

b. Which modifications (addition, change of attributes, operations, and/or operations' contract) must be made in this (these) class(es)? 


\section{Appendix C Survey Questionnaire}

Levels of agreement (Likert Scale):

1 - Strongly agree 2 - Agree 3 - Not certain 4 -Disagree 5 - Strongly disagree

\section{C.1 Pre-lab Survey Questionnaire}

1. Have you ever designed a "real-world" software system?
a) Yes
b) No

If "yes" to question 1), please answer question 1.1, otherwise jump to question 2.

1.1 How long have you worked on any "real-world" software systems accumulatively?

$$
\begin{aligned}
& <3 \text { months } \\
& >=3 \text { months, and }<6 \text { months } \\
& >=6 \text { months, and }<12 \text { months } \\
& >=12 \text { months }
\end{aligned}
$$

2. Did you have UML knowledge before this course?
a) Yes
b) No

If "yes" to question 3), please answer question 2.1 and 2.2, otherwise jump to question 3.

2.1 Have you ever used UML in analyzing / designing "real-world" software systems?
a) Yes
b) No

2.2 Which of the following statements is most appropriate to describe your experience of using UML?

a) I was introduced to UML in this course for the first time.

b) I have learned UML in school, but never applied it to a "real-world" system analysis.

c) I have used UML in analyzing "real-world" systems for just a few times.

d) I am very experienced in using UML and apply it to analyze "real-world" systems often.

e) None of the above, please specify:

3. Did you have any knowledge of OCL before this course?

a) Yes

b) No

If "no" to question 3, please skip the rest of the questions, otherwise continue.

3.1 How did you gain the knowledge of OCL?
a) I was taught in school.
b) I learned it by myself.
c) I was taught by co-workers.
d) None of the above, please specify

3.2 Have you ever used OCL in analyzing a system?

a) Never.

b) Yes, I have used it in my assignment at school only.

c) Yes, I have used it in analyzing "real-world" (commercial) systems. 
d) None of the above, please specify

\section{C.2 Defect Detection Survey Questionnaire}

\section{C.2.1 Common questions}

\begin{tabular}{|c|c|c|c|c|}
\hline & 1 & 2 & 3 & 4 \\
\hline 1. I had enough time to read through the analysis document. [] & $\square$ & $\square$ & $\square$ & $\square$ \\
\hline 2. I had no problems to find errors in the document. & $\square$ & $\square$ & $\square$ & $\square$ \\
\hline \multicolumn{2}{|c|}{ 3. I would have found more errors if there would have been more time. $\square$} & $\square$ & {[]} & [] \\
\hline 4. I fully understood what the system is trying to do. & $\square$ & $\square$ & $\square$ & $\square$ \\
\hline 5. I was not sure what type of errors I was looking for. & $\square$ & [ $]$ & $\square$ & $\square$ \\
\hline 6. The system analysis was fairly complex. & $\square$ & $\square$ & $\square$ & $\square$ \\
\hline 7. The lab instruction is clear and easy to follow. & $\square$ & $\square$ & $\square$ & $\square$ \\
\hline
\end{tabular}

\section{C.2.2 Extra questions for OCL groups}

$\begin{array}{lllll} & 1 & 2 & 3 & 4\end{array}$
10.How much time did you spend in understanding the OCL expressions during this lab?
A. $<25 \%$
B. $>=25 \%$ and $<50 \%$
C. $>=50 \%$ and $<75 \%$
D: $>=75 \%$

\section{D.3 Comprehension and Maintenance Survey Questionnaire}

\section{D.3.1 Common questions}

1. It took me a lot of time to understand the system, before I could answer any questions.

2. I did not have any problems to understand what the system is doing. $\square$ 
3. I had no difficulties to complete all the questions in this module.

$$
\begin{aligned}
& \text { Comprehension } \\
& \text { Maintenance }
\end{aligned}
$$

4. I hope there could have been more time for me to finish this module.

Comprehension

Maintenance

5. Some of the questions of this module were not easy to answer.

Comprehension

Maintenance

6. The lab instruction is clear and easy to follow.

\section{D.3.2 Extra questions for OCL groups}

7. The OCL expressions in the document were easy to understand.

8. The OCL expressions in the document made the system easier to understand.

9. The OCL expression in the document helped to clear up some ambiguities.

10. The OCL expression in the document helped me to answer the questions in this module.

$$
\begin{aligned}
& \text { Comprehension } \\
& \text { Maintenance }
\end{aligned}
$$

11. How much time did you spend in understanding the OCL expressions during this lab?
A. $<25 \%$
B. $>=25 \%$ and $<50 \%$
C. $>=50 \%$ and $<75 \%$
D: $>=75 \%$

\section{D.3.3 Extra questions for Lab 4 (last lab)}

12. Video system is more complex than Cab system

13. I would like to use $\mathrm{OCL}$ in my future system analyses.

Specify the reasons: 


\section{Appendix D Survey Results}

This appendix presents the survey results. The survey feedback was provided on a Likert scale [15]:

1. Strongly agree

2. Agree

3. Not certain

4. Disagree

5. Strongly disagree

The exception is question 10 of Defect Detection survey, and question 11 of Comprehension and Maintenance survey. The subjects' feedback of how time they spent on OCL was provided on the following scale:

1. $<25 \%$ of the lab time

2. $25 \sim 50 \%$ of the lab time

3. $50 \sim 75 \%$ of the lab time

4. $75 \sim 100 \%$ of the lab time 
Table 80. Defect Detection-1-I by Ability

\begin{tabular}{|l|l|c|c|c|c|}
\hline & High & Low & $\begin{array}{c}\text { p-value (t- } \\
\text { test) }\end{array}$ & $\begin{array}{c}\text { p-value (Wilconxon } \\
\text { test) }\end{array}$ \\
\hline 1 & Enough time & 3 & 2.94 & 0.4534 & 0.4919 \\
\hline 2 & No problem to find error & 3.2 & 3.19 & 0.4862 & 0.4671 \\
\hline 3 & Found more if more time & 2.4 & 2.19 & 0.3175 & 0.4103 \\
\hline 4 & Understand the system & 1.8 & 1.88 & 0.4138 & 0.3265 \\
\hline 5 & Not know what to look for & 3.2 & 2.69 & 0.10485 & 0.09875 \\
\hline 6 & System analysis is complex & 2.4 & 2.75 & 0.18295 & 0.1646 \\
\hline 7 & Instructions clear & 1.8 & 1.88 & 0.39085 & 0.22645 \\
\hline & & High & Low & & \\
\hline 8 & OCL easy to understand & 3 & 2.67 & 0.273 & 0.32885 \\
\hline 9 & OCL helped & 2.71 & 3.33 & 0.05695 & 0.06315 \\
\hline 10 & Time spent & 1.57 & 1.56 & 0.48665 & 0.33365 \\
\hline
\end{tabular}

Table 81. Defect Detection-3-I by Ability \& Method

\begin{tabular}{|c|l|c|c|c|c|c|c|c|c|}
\hline & & \multicolumn{4}{|c|}{ High ability } & \multicolumn{5}{c|}{ Low ability } \\
\hline & & OCL & no_OCL & $\begin{array}{c}\text { p-value (t } \\
\text { test) }\end{array}$ & $\begin{array}{c}\text { p-value } \\
\text { (Wilcoxon) }\end{array}$ & OCL & no_OCL & $\begin{array}{c}\text { p-value (t } \\
\text { test) }\end{array}$ & $\begin{array}{c}\text { p-value } \\
\text { (Wilcoxon) }\end{array}$ \\
\hline 1 & Enough time & 2.83 & 1.8 & 0.1269 & 0.1095 & 2.86 & 2.14 & 0.08165 & 0.07955 \\
\hline 2 & No problem to find error & 2.67 & 2.6 & 0.4500 & 0.4203 & 3.29 & 2.43 & 0.0757 & 0.09205 \\
\hline 3 & Found more if more time & 2.67 & 3.4 & 0.1828 & 0.1491 & 2.29 & 2.29 & 0.5 & 0.4736 \\
\hline 4 & Understand the system & 2.17 & 2 & 0.4038 & 0.3432 & 2 & 1.86 & 0.3409 & 0.38205 \\
\hline 5 & Not know what to look for & 3.83 & 2.6 & $\mathbf{0 . 0 2 9 8}$ & $\mathbf{0 . 0 4 2 4}$ & 3.29 & 3.71 & 0.18455 & 0.1664 \\
\hline 6 & System analysis is complex & 2.5 & 2.2 & 0.2459 & 0.3069 & 2.14 & 3.29 & $\mathbf{0 . 0 2 8 1}$ & $\mathbf{0 . 0 3 5 1}$ \\
\hline 7 & Instructions clear & 2 & 1.6 & 0.1484 & 0.1677 & 2.14 & 1.71 & 0.1448 & 0.14875 \\
\hline & & High & Low & p-value (t & $\begin{array}{c}\mathrm{p} \text {-value } \\
\text { test) }\end{array}$ & & & & \\
\hline & & 3 & 3.29 & 0.32555 & 0.2748 & & & & \\
\hline 8 & OCL easy to understand & 2.67 & 2.71 & 0.46265 & 0.4067 & & & & \\
\hline 9 & OCL helped & 2.17 & 2.67 & 0.2253 & 0.2279 & & & & \\
\hline 10 & Time spent & & & & & & & & \\
\hline
\end{tabular}


Table 83. Defect Detection-3-I by System

\begin{tabular}{|l|l|c|c|c|c|}
\hline \multicolumn{2}{|l|}{} & Cab & Video & $\begin{array}{c}\text { p-value (t) } \\
\text { test })\end{array}$ & $\begin{array}{c}\text { p-value } \\
\text { (Wilcoxon) }\end{array}$ \\
\hline 1 & Enough time & 2.38 & 3.39 & $\mathbf{0 . 0 2 7 7}$ & $\mathbf{0 . 0 2 9 5}$ \\
\hline 2 & No problem to find error & 2.92 & 3.39 & 0.0984 & 0.0690 \\
\hline 3 & Found more if more time & 2.54 & 2.11 & 0.1715 & 0.1015 \\
\hline 4 & Understand the system & 1.85 & 1.83 & 0.4854 & 0.4058 \\
\hline 5 & Not know what to look for & 3.15 & 2.78 & 0.1833 & 0.1884 \\
\hline 6 & System analysis is complex & 2.92 & 2.33 & 0.0638 & 0.0527 \\
\hline 7 & Instructions clear & 1.69 & 1.94 & 0.1772 & 0.2584 \\
\hline & & Cab & Video & & \\
\hline 8 & OCL easy to understand & 3.5 & 2.4 & $\mathbf{0 . 0 1 8 3}$ & $\mathbf{0 . 0 2 6 9}$ \\
\hline 9 & OCL helped & 3.17 & 3 & 0.3454 & 0.3423 \\
\hline 10 & Time spent & 1.67 & 1.5 & 0.3654 & 0.5000 \\
\hline
\end{tabular}

Table 83. Comparing Defect Detection-1-I and Defect Detection-3-I

\begin{tabular}{|c|l|c|c|c|}
\hline & & Lab 1 & Lab 3 & p-value \\
\hline 1 & Had enough time & 2.96 & 2.44 & 0.0569 \\
\hline 2 & No problems to find defects & 3.2 & 2.76 & 0.0887 \\
\hline 3 & More found if more time & 2.24 & 2.6 & 0.925 \\
\hline 4 & Understood the system & 1.88 & 2 & 0.2391 \\
\hline 5 & Not sure what type of defects & 2.96 & 3.4 & $\mathbf{0 . 0 2 6 7}$ \\
\hline 6 & System analysis complex & 2.48 & 2.56 & 0.3959 \\
\hline 7 & Instruction clear & 1.84 & 1.88 & 0.3939 \\
\hline
\end{tabular}

Table 85. Comparing D-1-I and D-3-I - attitudes of OCL

\begin{tabular}{|c|l|c|c|c|c|}
\hline & & Lab 1 & Lab 3 & p-value (t test) & p-value (Wilcoxon) \\
\hline 8 & OCL easy to understand & 2.81 & 3.15 & 0.1972 & 0.2176 \\
\hline 9 & OCL helped & 3.06 & 2.69 & 0.1157 & 0.1083 \\
\hline 10 & Time spent & 1.56 & 2.42 & $\mathbf{0 . 0 1 5 3}$ & $\mathbf{0 . 0 1 5 6}$ \\
\hline
\end{tabular}


Table 85. Comparing D-1-I and D-3-I by Ability

\begin{tabular}{|c|l|c|c|c|c|c|c|}
\hline & & \multicolumn{3}{|c|}{ High ability } & \multicolumn{3}{c|}{ Low ability } \\
\hline & & Lab 1 & Lab 3 & p-value & Lab 1 & Lab 3 & p-value \\
\hline 1 & Enough time & 3.1 & 2.36 & $\mathbf{0 . 0 2 6 9}$ & 2.86 & 2.5 & 0.2478 \\
\hline 2 & No problem to find error & 3.09 & 2.64 & 0.1354 & 3.29 & 2.86 & 0.1981 \\
\hline 3 & Found more if more time & 2.27 & 3 & $\mathbf{0 . 0 3 5 2}$ & 2.21 & 2.29 & 0.425 \\
\hline 4 & Understand the system & 1.91 & 2.09 & 0.2764 & 1.86 & 1.93 & 0.3601 \\
\hline 5 & Not know what to look for & 3.36 & 3.27 & 0.338 & 2.64 & 3.5 & $\mathbf{0 . 0 0 8 3}$ \\
\hline 6 & System analysis is complex & 2.18 & 2.36 & 0.2942 & 2.71 & 2.71 & 0.5 \\
\hline 7 & Instructions clear & 1.82 & 1.82 & 0.5 & 1.86 & 1.93 & 0.3601 \\
\hline
\end{tabular}

Table 86. Comparing D-1-I and D-3-I - attitudes of OCL by Ability

\begin{tabular}{|l|l|c|c|c|c|c|c|c|c|}
\hline & & \multicolumn{4}{|c|}{ High ability } & \multicolumn{4}{c|}{ Low ability } \\
\hline & & Lab 1 & Lab 3 & $\begin{array}{c}\text { p-value (t } \\
\text { test) }\end{array}$ & $\begin{array}{c}\text { p-value } \\
\text { (Wilcoxon) }\end{array}$ & Lab 1 & Lab 3 & $\begin{array}{c}\text { p-value (t } \\
\text { test) }\end{array}$ & $\begin{array}{c}\mathrm{p} \text {-value } \\
\text { (Wilcoxon) }\end{array}$ \\
\hline 8 & OCL easy to understand & 3 & 3 & 0.5000 & 0.4705 & 2.67 & 3.29 & 0.0783 & 0.0928 \\
\hline 9 & OCL helped & 2.71 & 2.67 & 0.4627 & 0.4067 & 3.33 & 2.71 & 0.0570 & 0.0632 \\
\hline 10 & Time spent & 1.57 & 2.17 & 0.1861 & 0.1366 & 1.56 & 2.67 & $\mathbf{0 . 0 1 4 4}$ & $\mathbf{0 . 0 2 3 6}$ \\
\hline
\end{tabular}

Table 87. Defect Detection-1-II by Ability

\begin{tabular}{|c|l|c|c|c|c|}
\hline & & High & Low & p-value (t-test) & p-value (Wilconxon test) \\
\hline 1 & Enough time & 3.26 & 3.27 & 0.4860 & 0.4522 \\
\hline 2 & No problem to find error & 2.94 & 3.4 & $\mathbf{0 . 0 2 0 8}$ & $\mathbf{0 . 0 1 4 3}$ \\
\hline 3 & Found more if more time & 2.11 & 2.16 & 0.4331 & 0.3901 \\
\hline 4 & Understand the system & 2 & 2.09 & 0.3293 & 0.3773 \\
\hline 5 & Not know what to look for & 3.14 & 2.42 & $\mathbf{0 . 0 0 2 9}$ & $\mathbf{0 . 0 0 3 2}$ \\
\hline 6 & System analysis is complex & 2.63 & 2.33 & 0.0826 & 0.0641 \\
\hline 7 & Instructions clear & 2 & 2.27 & 0.1243 & 0.2215 \\
\hline & & High & Low & & \\
\hline 8 & OCL easy to understand & 3.24 & 3.17 & 0.4248 & 0.3931 \\
\hline 9 & OCL helped & 3.31 & 2.96 & 0.1908 & 0.1969 \\
\hline 10 & Time spent & 1.59 & 2.09 & $\mathbf{0 . 0 3 3 4}$ & $\mathbf{0 . 0 1 6 0}$ \\
\hline
\end{tabular}


Table 89. Defect Detection-1-II by System

\begin{tabular}{|c|l|c|c|c|c|}
\hline & & CD & VS & p-value (t-test) & p-value (Wilconxon test) \\
\hline 1 & Enough time & 2.95 & 3.55 & $\mathbf{0 . 0 1 1 5}$ & $\mathbf{0 . 0 1 1 0}$ \\
\hline 2 & No problem to find error & 3.13 & 3.26 & 0.2817 & 0.2900 \\
\hline 3 & Found more if more time & 2.29 & 2 & 0.1160 & 0.1359 \\
\hline 4 & Understand the system & 1.95 & 2.14 & 0.1634 & 0.0758 \\
\hline 5 & Not know what to look for & 2.71 & 2.76 & 0.4235 & 0.4565 \\
\hline 6 & System analysis is complex & 2.61 & 2.33 & 0.0993 & 0.1025 \\
\hline 7 & Instructions clear & 2.03 & 2.26 & 0.1527 & 0.1234 \\
\hline 8 & OCL easy to understand & 3.11 & 3.29 & 0.2863 & 0.2718 \\
\hline 9 & OCL helped & 2.79 & 3.4 & 0.0616 & 0.0608 \\
\hline 10 & Time spent & 1.95 & 1.81 & 0.3081 & 0.2805 \\
\hline
\end{tabular}

Table 89 Defect Detection-3-II by Method

\begin{tabular}{|c|l|c|c|c|c|}
\hline & & OCL & no_OCL & p-value (t test) & p-value (Wilcoxon) \\
\hline 1 & Enough time & 2.08 & 2.1 & 0.46975 & 0.42545 \\
\hline 2 & No problem to find error & 2.74 & 2.67 & 0.36815 & 0.3428 \\
\hline 3 & Found more if more time & 3 & 3.12 & 0.31285 & 0.29005 \\
\hline 4 & Understand the system & 2.03 & 1.95 & 0.34125 & 0.4025 \\
\hline 5 & Not know what to look for & 2.95 & 3.02 & 0.3851 & 0.33725 \\
\hline 6 & System analysis is complex & 2.67 & 2.9 & 0.15435 & 0.16795 \\
\hline 7 & Instructions clear & 1.97 & 2.07 & 0.3364 & 0.29745 \\
\hline
\end{tabular}

Table 91 Defect Detection-3-II by Ability

\begin{tabular}{|c|l|c|c|c|c|}
\hline & & $\begin{array}{c}\text { High } \\
\text { ability }\end{array}$ & $\begin{array}{c}\text { Low } \\
\text { ability }\end{array}$ & $\begin{array}{c}\text { p-value (t } \\
\text { test) }\end{array}$ & $\begin{array}{c}\text { p-value } \\
\text { (Wilcoxon) }\end{array}$ \\
\hline 1 & Enough time & 2.32 & 1.89 & $\mathbf{0 . 0 3 3 7}$ & $\mathbf{0 . 0 2 8 4}$ \\
\hline 2 & No problem to find error & 2.68 & 2.73 & 0.41095 & 0.46055 \\
\hline 3 & Found more if more time & 3.08 & 3.05 & 0.4422 & 0.4531 \\
\hline 4 & Understand the system & 1.92 & 2.05 & 0.2404 & 0.30215 \\
\hline 5 & Not know what to look for & 3.24 & 2.77 & $\mathbf{0 . 0 3 2 6}$ & $\mathbf{0 . 0 3 6 5}$ \\
\hline 6 & System analysis is complex & 2.78 & 2.8 & 0.48025 & 0.451 \\
\hline 7 & Instructions clear & 2 & 2.05 & 0.4219 & 0.37525 \\
\hline 8 & OCL easy to understand & 2.89 & 2.9 & 0.4871 & 0.4332 \\
\hline 9 & OCL helped & 2.56 & 3.05 & 0.0668 & $\mathbf{0 . 0 4 7}$ \\
\hline 10 & Time spent & 1.83 & 2 & 0.2904 & 0.2709 \\
\hline
\end{tabular}


Table 93 Defect Detection-3-II - attitudes of OCL by Ability

\begin{tabular}{|c|l|c|c|c|c|}
\hline & & High & Low & $\begin{array}{c}\text { p-value }(t \\
\text { test) }\end{array}$ & $\begin{array}{c}\text { p-value } \\
\text { (Wilcoxon) }\end{array}$ \\
\hline 8 & OCL easy to understand & 2.89 & 2.9 & 0.4871 & 0.4332 \\
\hline 9 & OCL helped & 2.56 & 3.05 & 0.0668 & $\mathbf{0 . 0 4 7}$ \\
\hline 10 & Time spent & 1.83 & 2 & 0.2904 & 0.2709 \\
\hline
\end{tabular}

Table 92 Comparing Defect Detection-1-II and Defect Detection-3-II

\begin{tabular}{|c|l|c|c|c|}
\hline & & Lab 1 & Lab 3 & p-value \\
\hline 1 & Had enough time & 3.27 & 2.08 & $<.0001$ \\
\hline 2 & No problems to find defects & 3.2 & 2.68 & $<.0001$ \\
\hline 3 & More found if more time & 2.13 & 3.1 & $<.0001$ \\
\hline 4 & Understood the system & 2.06 & 1.99 & 0.2384 \\
\hline 5 & Not sure what type of defects & 2.75 & 3.02 & $\mathbf{0 . 0 3 1 3}$ \\
\hline 6 & System analysis complex & 2.46 & 2.81 & $\mathbf{0 . 0 1 1 1}$ \\
\hline 7 & Instruction clear & 2.16 & 2.04 & 0.1361 \\
\hline
\end{tabular}

Table 93 Comparing D-1-II and D-3-II - attitudes of OCL

\begin{tabular}{|c|l|c|c|c|c|}
\hline & & Lab 1 & Lab 3 & $\begin{array}{c}\text { p-value } \\
\text { (Wilcoxon) }\end{array}$ & $\begin{array}{c}\text { p-value (t } \\
\text { test) }\end{array}$ \\
\hline 8 & OCL easy to understand & 3.2 & 2.89 & 0.0989 & 0.0937 \\
\hline 9 & OCL helped & 3.1 & 2.81 & 0.1306 & 0.134 \\
\hline 10 & Time spent & 1.88 & 1.92 & 0.4467 & 0.4092 \\
\hline
\end{tabular}

Table 95 Comparing D-1-II and D-3-II by Ability

\begin{tabular}{|c|l|c|c|c|c|c|c|}
\hline & & \multicolumn{3}{|c|}{ High ability } & \multicolumn{3}{c|}{ Low ability } \\
\hline & & Lab 1 & Lab 3 & p-value & Lab 1 & Lab 3 & p-value \\
\hline 1 & Enough time & 3.26 & 2.31 & $\mathbf{0 . 0 0 0 6}$ & 3.27 & 1.89 & $<.0001$ \\
\hline 2 & No problem to find error & 2.94 & 2.63 & 0.0775 & 3.41 & 2.73 & $<.0001$ \\
\hline 3 & Found more if more time & 2.11 & 3.17 & $<.0001$ & 2.14 & 3.05 & $<.0001$ \\
\hline 4 & Understand the system & 2 & 1.91 & 0.3231 & 2.11 & 2.05 & 0.292 \\
\hline 5 & Not know what to look for & 3.14 & 3.34 & 0.1807 & 2.43 & 2.77 & $\mathbf{0 . 0 5}$ \\
\hline 6 & System analysis is complex & 2.63 & 2.83 & 0.2001 & 2.32 & 2.8 & $\mathbf{0 . 0 1 1}$ \\
\hline 7 & Instructions clear & 2 & 2.03 & 0.4302 & 2.3 & 2.05 & 0.062 \\
\hline
\end{tabular}


Table 96 Comparing D-1-II and D-3-II - attitudes of OCL by Ability

\begin{tabular}{|c|l|c|c|c|c|c|c|c|c|}
\hline & & \multicolumn{4}{|c|}{ High ability } & \multicolumn{4}{c|}{ Low ability } \\
\hline & & Lab 1 & Lab 3 & $\begin{array}{c}\text { p-value (t } \\
\text { test) }\end{array}$ & $\begin{array}{c}\text { p-value } \\
\text { (Wilcoxon) }\end{array}$ & Lab 1 & Lab 3 & $\begin{array}{c}\text { p-value (t } \\
\text { test) }\end{array}$ & $\begin{array}{c}\mathrm{p} \text {-value } \\
\text { (Wilcoxon) }\end{array}$ \\
\hline 8 & OCL easy to understand & 3.24 & 2.89 & 0.1406 & 0.1017 & 3.17 & 2.9 & 0.2089 & 0.2473 \\
\hline 9 & OCL helped & 3.31 & 2.56 & $\mathbf{0 . 0 2 8 6}$ & $\mathbf{0 . 0 3 1 4}$ & 2.96 & 3.05 & 0.3936 & 0.4152 \\
\hline 10 & Time spent & 1.59 & 1.83 & 0.2128 & 0.1877 & 2.09 & 2 & 0.3703 & 0.2936 \\
\hline
\end{tabular}

Table 96. Comprehesnion-2-I and Maintenance-2-I by Method

\begin{tabular}{|c|l|c|c|c|c|}
\hline & & OCL & No_OCL & $\begin{array}{c}\text { p-value (t } \\
\text { test) }\end{array}$ & $\begin{array}{c}\mathrm{p} \text {-value } \\
\text { (Wilcoxon) }\end{array}$ \\
\hline 1 & A lot of time to understand & 2.87 & 2.82 & 0.4604 & 0.5000 \\
\hline 2 & No problem to understand & 2.53 & 2.24 & 0.2326 & 0.1029 \\
\hline $3 \mathrm{C}^{3}$ & No difficulties to complete & 2.57 & 2.82 & 0.2485 & 0.3415 \\
\hline $3 \mathrm{M}$ & No difficulties to complete & 3 & 3.24 & 0.2718 & 0.3259 \\
\hline 4C & Hope more time & 3.2 & 3 & 0.3390 & 0.3413 \\
\hline 4M & Hope more time & 3.13 & 2.59 & 0.1255 & 0.1309 \\
\hline $5 \mathrm{C}$ & Question not easy & 2.4 & 2.65 & 0.2436 & 0.2680 \\
\hline $5 \mathrm{M}$ & Question not easy & 2.6 & 2.53 & 0.4215 & 0.4750 \\
\hline 6 & Instructions clear & 2 & 2.12 & 0.3706 & 0.4350 \\
\hline
\end{tabular}

\footnotetext{
${ }^{3}$ There are some questions asked for each task. C denotes the question was answered in the context of Comprehension task; $\mathrm{M}$ denotes the question was answered in the context of Maintenance task.
} 
Table 97. Comprehesnion-2-I and Maintenance-2-I by System

\begin{tabular}{|c|c|c|c|c|c|}
\hline & & $\mathrm{Cab}$ & Video & $\mathrm{p}$-value ( $\mathrm{t}$ test) & p-value (Wilcoxon) \\
\hline 1 & A lot of time to understand & 2.93 & 2.76 & 0.3485 & 0.3551 \\
\hline 2 & No problem to understand & 2.33 & 2.41 & 0.4241 & 0.4294 \\
\hline $3 \mathrm{C}$ & \multirow[t]{2}{*}{ No difficulties to complete } & 2.8 & 2.63 & 0.3183 & 0.3422 \\
\hline $3 \mathrm{M}$ & & 3.27 & 3 & 0.2454 & 0.2981 \\
\hline $4 \mathrm{C}$ & \multirow[t]{2}{*}{ Hope more time } & 3.2 & 3 & 0.3390 & 0.3202 \\
\hline $4 \mathrm{M}$ & & 2.53 & 3.12 & 0.1089 & 0.0726 \\
\hline $5 \mathrm{C}$ & \multirow[t]{2}{*}{ Question not easy } & 2.93 & 2.18 & 0.0136 & 0.0189 \\
\hline $5 \mathrm{M}$ & & 2.33 & 2.76 & 0.1102 & 0.0900 \\
\hline 6 & Instructions clear & 1.87 & 2.24 & 0.1483 & 0.2765 \\
\hline 7 & OCL easy to understand & 3.33 & 2.57 & 0.0996 & 0.1270 \\
\hline 8 & OCL made the system easier & 2.67 & 2.14 & 0.1745 & 0.2829 \\
\hline 9 & OCL cleared up ambiguities & 2.6 & 1.86 & 0.0671 & 0.0863 \\
\hline $10 \mathrm{C}$ & \multirow[t]{2}{*}{ OCL helped } & 1.83 & 2 & 0.3722 & 0.4686 \\
\hline $10 \mathrm{M}$ & & 3.33 & 3.14 & 0.3644 & 0.3263 \\
\hline 11 & Time spent & 2.17 & 1.71 & 0.1521 & 0.1591 \\
\hline
\end{tabular}

Table 98. Comprehesnion-4-I and Maintenance-4-I by Method

\begin{tabular}{|c|l|c|c|c|c|}
\hline & & OCL & No_OCL & p-value (t test) & p-value (Wilcoxon) \\
\hline 1 & A lot of time to understand & 3.87 & 2.86 & $\mathbf{0 . 0 0 6 0}$ & $\mathbf{0 . 0 0 7 0}$ \\
\hline 2 & No problem to understand & 2.07 & 2.71 & 0.0600 & $\mathbf{0 . 0 4 7 6}$ \\
\hline 3C & No difficulties to complete & 2.5 & 2.62 & 0.3869 & 0.3577 \\
\hline 3M & No difficulties to complete & 2.4 & 2.71 & 0.1700 & 0.1287 \\
\hline 4C & Hope more time & 3.29 & 3.25 & 0.4686 & 0.4243 \\
\hline 4M & Hope more time & 2.93 & 3.08 & 0.3838 & 0.3964 \\
\hline 5C & Question not easy & 3.5 & 1.92 & $\mathbf{0 . 0 0 0 2}$ & $\mathbf{0 . 0 0 0 7}$ \\
\hline $5 \mathrm{M}$ & Question not easy & 2.8 & 2 & $\mathbf{0 . 0 0 9 9}$ & $\mathbf{0 . 0 0 9 4}$ \\
\hline 6 & Instructions clear & 1.6 & 1.64 & 0.4446 & 0.4654 \\
\hline
\end{tabular}


Table 100. Comprehesnion-4-I and Maintenance-4-I by Ability

\begin{tabular}{|c|l|c|c|c|c|}
\hline & & High Ability & Low Ability & p-value (t test) & p-value (Wilcoxon) \\
\hline 1 & A lot of time to understand & 3.53 & 3.21 & 0.2258 & 0.2020 \\
\hline 2 & No problem to understand & 2.27 & 2.5 & 0.2914 & 0.2865 \\
\hline $3 \mathrm{C}$ & No difficulties to complete & 2.38 & 2.71 & 0.2044 & 0.1950 \\
\hline $4 \mathrm{C}$ & Hope more time & 3.33 & 3.21 & 0.3962 & 0.3116 \\
\hline 5C & Question not easy & 2.92 & 2.57 & 0.2395 & 0.2651 \\
\hline $3 \mathrm{M}$ & No difficulties to complete & 2.47 & 2.64 & 0.2974 & 0.3745 \\
\hline $4 \mathrm{M}$ & Hope more time & 3.07 & 2.93 & 0.3841 & 0.3875 \\
\hline $5 \mathrm{M}$ & Question not easy & 2.27 & 2.57 & 0.1978 & 0.1421 \\
\hline 6 & Instructions clear & 1.46 & 1.77 & 0.1127 & 0.1451 \\
\hline 12 & Video more complex & 2.73 & 2.38 & 0.2142 & 0.2727 \\
\hline 13 & Use OCL in the future & 2.53 & 2.23 & 0.2070 & 0.3423 \\
\hline & & High ability & Low ability & & \\
\hline 7 & OCL easy to understand & 2.38 & 2.67 & 0.3081 & 0.2935 \\
\hline 8 & OCL made the system easier & 2 & 2 & 0.5000 & 0.4170 \\
\hline 9 & OCL cleared up ambiguities & 2.13 & 1.8 & 0.2956 & 0.3504 \\
\hline $10 \mathrm{C}$ & OCL helped & 1.86 & 2.17 & 0.2278 & 0.2395 \\
\hline $10 \mathrm{M}$ & OCL helped & 2.38 & 2.67 & 0.3200 & 0.4459 \\
\hline 11 & Time spent & 2 & 2 & 0.5000 & 0.4699 \\
\hline
\end{tabular}

Table 102. Comprehesnion-4-I and Maintenance-4-I by System

\begin{tabular}{|c|l|c|c|c|c|}
\hline & & $\mathrm{CD}$ & $\mathrm{VS}$ & $\mathrm{p}$-value (t test) & $\mathrm{p}$-value (Wilcoxon) \\
\hline 1 & A lot of time to understand & 3.41 & 3.33 & 0.4280 & 0.4184 \\
\hline 2 & No problem to understand & 2.35 & 2.42 & 0.4414 & 0.4184 \\
\hline $3 \mathrm{C}$ & No difficulties to complete & 2.59 & 2.5 & 0.4159 & 0.3858 \\
\hline $3 \mathrm{M}$ & No difficulties to complete & 2.65 & 2.42 & 0.2461 & 0.2194 \\
\hline 4C & Hope more time & 3.24 & 3.33 & 0.4181 & 0.3766 \\
\hline 4M & Hope more time & 2.76 & 3.36 & 0.1105 & 0.1067 \\
\hline $5 \mathrm{C}$ & Question not easy & 3 & 2.3 & 0.0836 & 0.0883 \\
\hline $5 \mathrm{M}$ & Question not easy & 2.53 & 2.25 & 0.2216 & 0.2392 \\
\hline 6 & Instructions clear & 1.53 & 1.73 & 0.2273 & 0.1422 \\
\hline & & $\mathrm{CD}$ & $\mathrm{VS}$ & & \\
\hline 7 & OCL easy to understand & 1.88 & 3.33 & $\mathbf{0 . 0 0 1 4}$ & 0.0050 \\
\hline 8 & OCL made the system easier & 1.63 & 2.5 & $\mathbf{0 . 0 3 0 5}$ & 0.0467 \\
\hline 9 & OCL cleared up ambiguities & 1.75 & 2.4 & $\mathbf{0 . 1 3 6 1}$ & 0.1989 \\
\hline $10 \mathrm{C}$ & OCL helped & 1.88 & 2.2 & 0.2221 & 0.2341 \\
\hline $10 \mathrm{M}$ & OCL helped & 2.13 & 3 & 0.0719 & 0.1236 \\
\hline 11 & Time spent & 2 & 2 & 0.5000 & 0.4699 \\
\hline
\end{tabular}


Table 101. Comparing 2-I and 4-I

\begin{tabular}{|c|l|c|c|c|}
\hline & & Lab 2 & Lab 4 & p-value \\
\hline 1 & A lot of time to understand the system & 2.86 & 3.38 & 0.0615 \\
\hline 2 & No problems to understand & 2.34 & 2.4 & 0.4395 \\
\hline 3C & No difficulties to complete Comprehension & 2.81 & 2.5 & 0.1119 \\
\hline 3M & No difficulties to complete Maintenance & 3.1 & 2.55 & $\mathbf{0 . 0 1 1 8}$ \\
\hline 4C & Hope more time for Comprehension & 2.96 & 3.27 & 0.0938 \\
\hline 4M & Hope more time for Maintenance & 2.89 & 3 & 0.3467 \\
\hline $5 \mathrm{C}$ & Questions not easy in Comprehension & 2.37 & 2.74 & 0.105 \\
\hline $5 \mathrm{M}$ & Questions not easy in Maintenance & 2.55 & 2.41 & 0.2732 \\
\hline 6 & Instruction clear & 2.12 & 1.62 & $\mathbf{0 . 0 2 2 5}$ \\
\hline
\end{tabular}

Table 103. Comparing 2-I and 4-I - attitudes of OCL

\begin{tabular}{|c|l|c|c|c|c|}
\hline & & Lab 2 & Lab 4 & p-value ( $t$ test) & p-value (Wilcoxon) \\
\hline 7 & OCL easy to understand & 2.92 & 2.5 & 0.1478 & 0.1708 \\
\hline 8 & OCL made the system easier & 2.38 & 2 & 0.1436 & 0.1355 \\
\hline 9 & OCL cleared up ambiguities & 2.17 & 2 & 0.3284 & 0.3015 \\
\hline $10 \mathrm{C}$ & OCL helped - Comprehension & 1.92 & 2 & 0.4029 & 0.3255 \\
\hline $10 \mathrm{M}$ & OCL helped - Maintenance & 3.23 & 2.5 & $\mathbf{0 . 0 3 6 8}$ & $\mathbf{0 . 0 3 0 6}$ \\
\hline 11 & Time spent & 1.92 & 2 & 0.4029 & 0.4133 \\
\hline
\end{tabular}

Table 105. Comparing 2-I and 4-I by Ability

\begin{tabular}{|c|l|c|c|c|c|c|c|}
\hline & & \multicolumn{3}{|c|}{ High ability } & \multicolumn{3}{c|}{ Low ability } \\
\hline & & Lab 2 & Lab 4 & p-value & Lab 2 & Lab 4 & p-value \\
\hline 1 & A lot of time to understand & 3.2 & 3.53 & 0.1767 & 2.5 & 3.21 & 0.0532 \\
\hline 2 & No problem to understand & 2.6 & 2.27 & 0.1671 & 2.07 & 2.5 & 0.0693 \\
\hline 3C & No difficulties to complete & 2.77 & 2.38 & 0.158 & 2.85 & 2.62 & 0.2567 \\
\hline 3M & No difficulties to complete & 3 & 2.47 & 0.0749 & 3.21 & 2.64 & $\mathbf{0 . 0 4 3 8}$ \\
\hline 4C & Hope more time & 3 & 3.29 & 0.207 & 2.92 & 3.25 & 0.1525 \\
\hline 4M & Hope more time & 2.31 & 3.07 & 0.3175 & 2.57 & 2.93 & 0.2219 \\
\hline $5 \mathrm{C}$ & Question not easy & 2.5 & 3.5 & $\mathbf{0 . 0 1 6 5}$ & 2.23 & 1.92 & 0.1685 \\
\hline $5 \mathrm{M}$ & Question not easy & 2.67 & 2.27 & $\mathbf{0 . 1 2 6 3}$ & 2.43 & 2.57 & 0.3175 \\
\hline 6 & Instructions clear & 2.15 & 1.46 & $\mathbf{0 . 0 2 8 2}$ & 2.08 & 1.77 & 0.1962 \\
\hline
\end{tabular}


Table 107. Comparing 2-I and 4-I - attitudes of OCL by Ability

\begin{tabular}{|c|l|c|c|c|c|c|c|c|c|}
\hline & & \multicolumn{4}{|c|}{ High ability } & \multicolumn{4}{c|}{ Low ability } \\
\hline & Lab 2 & Lab 4 & $\begin{array}{c}\text { p-value (t } \\
\text { test) }\end{array}$ & $\begin{array}{c}\text { p-value } \\
\text { (Wilconxon) }\end{array}$ & Lab 2 & Lab 4 & $\begin{array}{c}\text { p-value (t } \\
\text { test) }\end{array}$ & $\begin{array}{c}\text { p-value } \\
\text { (Wilcoxon) }\end{array}$ \\
\hline 7 & OCL easy to understand & 2.71 & 2.38 & 0.2781 & 0.2685 & 3.17 & 2.67 & 0.2053 & 0.2488 \\
\hline 8 & OCL made the system easier & 2.14 & 2 & 0.3837 & 0.3104 & 2.67 & 2 & 0.1298 & 0.1698 \\
\hline 9 & OCL cleared up ambiguities & 1.83 & 2.13 & 0.2973 & 0.3671 & 2.5 & 1.8 & 0.1002 & 0.1149 \\
\hline $10 \mathrm{C}$ & OCL helped & 1.86 & 1.86 & 0.5000 & 0.4728 & 2 & 2.17 & 0.2998 & 0.3367 \\
\hline $10 \mathrm{M}$ & OCL helped & 3.57 & 2.38 & $\mathbf{0 . 0 1 4 7}$ & $\mathbf{0 . 0 2 1 5}$ & 2.83 & 2.67 & 0.3995 & 0.3599 \\
\hline 11 & Time spent & 1.57 & 2 & $\mathbf{0 . 1 3 4 0}$ & 0.1632 & 2.33 & 2 & 0.2578 & 0.2753 \\
\hline
\end{tabular}

Table 105. Comprehesnion-2-II and Maintenance-2-II by Method

\begin{tabular}{|c|l|c|c|c|c|}
\hline & & OCL & no_OCL & p-value (t test) & p-value (Wilcoxon) \\
\hline 1 & A lot of time to understand & 3.15 & 2.9 & 0.1681 & 0.1455 \\
\hline 2 & No problem to understand & 2.6 & 2.83 & 0.1265 & 0.1365 \\
\hline 3C & No difficulties to complete & 2.8 & 2.95 & 0.2632 & 0.2685 \\
\hline 3M & No difficulties to complete & 2.85 & 2.95 & 0.3309 & 0.2860 \\
\hline 4C & Hope more time & 3.18 & 2.83 & 0.1400 & 0.1563 \\
\hline 4M & Hope more time & 3.3 & 2.95 & 0.1251 & 0.1401 \\
\hline 5C & Question not easy & 2.38 & 2.29 & 0.3389 & 0.3754 \\
\hline $5 \mathrm{M}$ & Question not easy & 2.48 & 2.41 & 0.3913 & 0.3828 \\
\hline 6 & Instructions clear & 2.03 & 2.17 & 0.2540 & 0.2873 \\
\hline
\end{tabular}

\section{Table 106. Comprehesnion-2-II and Maintenance-2-II by System}

\begin{tabular}{|c|l|c|c|c|c|}
\hline & & $\mathrm{CD}$ & $\mathrm{VS}$ & $\mathrm{p}$-value (t test) & $\mathrm{p}$-value (Wilcoxon) \\
\hline 1 & A lot of time to understand & 3.08 & 2.98 & 0.3483 & 0.2818 \\
\hline 2 & No problem to understand & 2.69 & 2.74 & 0.4101 & 0.3084 \\
\hline 3C & No difficulties to complete & 2.95 & 2.81 & 0.2801 & 0.2815 \\
\hline 3M & No difficulties to complete & 3.23 & 2.6 & $\mathbf{0 . 0 0 2 5}$ & $\mathbf{0 . 0 0 2 9}$ \\
\hline 4C & Hope more time & 2.82 & 3.17 & $\mathbf{0 . 1 3 9 9}$ & $\mathbf{0 . 1 7 5 7}$ \\
\hline 4M & Hope more time & 2.82 & 3.4 & $\mathbf{0 . 0 2 5 7}$ & $\mathbf{0 . 0 4 1 2}$ \\
\hline $5 \mathrm{C}$ & Question not easy & 2.51 & 2.17 & 0.0597 & 0.1125 \\
\hline $5 \mathrm{M}$ & Question not easy & 2.36 & 2.52 & 0.2252 & 0.1964 \\
\hline 6 & Instructions clear & 2.08 & 2.12 & 0.4243 & 0.2557 \\
\hline 7 & OCL easy to understand & 2.44 & 2.37 & 0.4035 & 0.2068 \\
\hline 8 & OCL made the system easier & 2.44 & 2.21 & 0.2541 & 0.1346 \\
\hline 9 & OCL cleared up ambiguities & 2 & 2.44 & 0.1033 & 0.2155 \\
\hline 10C & OCL helped & 1.88 & 1.79 & 0.3684 & 0.1348 \\
\hline 10M & OCL helped & 2.39 & 2.79 & 0.1409 & 0.1444 \\
\hline 11 & Time spent & 1.33 & 1.89 & $\mathbf{0 . 0 1 8 7}$ & $\mathbf{0 . 0 2 3 2}$ \\
\hline
\end{tabular}


Table 107. Comprehesnion-4-II and Maintenance-4-II by Method

\begin{tabular}{|c|l|c|c|c|c|}
\hline & & OCL & No_OCL & p-value (t test) & p-value (Wilcoxon) \\
\hline 1 & A lot of time to understand & 3.25 & 3.05 & 0.2214 & 0.2364 \\
\hline 2 & No problem to understand & 2.41 & 2.49 & 0.3722 & 0.2601 \\
\hline 3C & No difficulties to complete & 2.71 & 2.57 & 0.2692 & 0.3176 \\
\hline 3M & No difficulties to complete & 2.98 & 2.7 & 0.1173 & 0.1300 \\
\hline 4C & Hope more time & 3.22 & 3.73 & $\mathbf{0 . 0 1 6 9}$ & $\mathbf{0 . 0 2 4 5}$ \\
\hline 4M & Hope more time & 3.1 & 3.47 & 0.0720 & 0.0768 \\
\hline 5C & Question not easy & 2.95 & 2.3 & $\mathbf{0 . 0 0 2 8}$ & $\mathbf{0 . 0 0 3 8}$ \\
\hline 5M & Question not easy & 2.51 & 2.62 & 0.3165 & 0.2822 \\
\hline 6 & Instructions clear & 1.78 & 2.11 & 0.0824 & 0.2517 \\
\hline
\end{tabular}

Table 109. Comprehesnion-4-II and Maintenance-4-II by Ability

\begin{tabular}{|c|l|c|c|c|c|}
\hline & & High & Low & p-value (t test) & p-value (Wilcoxon) \\
\hline 1 & A lot of time to understand & 3.11 & 3.19 & 0.3865 & 0.4065 \\
\hline 2 & No problem to understand & 2.57 & 2.35 & 0.1564 & 0.1584 \\
\hline $3 \mathrm{C}$ & No difficulties to complete & 2.74 & 2.56 & 0.2088 & 0.2316 \\
\hline 3M & No difficulties to complete & 2.86 & 2.84 & 0.4657 & 0.4979 \\
\hline $4 \mathrm{C}$ & Hope more time & 3.46 & 3.47 & 0.4870 & 0.4381 \\
\hline 4M & Hope more time & 3.35 & 3.21 & 0.2898 & 0.3445 \\
\hline $5 \mathrm{C}$ & Question not easy & 2.6 & 2.67 & 0.3796 & 0.3548 \\
\hline $5 \mathrm{M}$ & Question not easy & 2.6 & 2.54 & 0.3886 & 0.4263 \\
\hline 6 & Instruction0.3014s clear & 2.14 & 1.77 & 0.0560 & $\mathbf{0 . 0 1 7 3}$ \\
\hline & & High & Low & & \\
\hline 7 & OCL easy to understand & 2.28 & 2.67 & 0.1146 & 0.0801 \\
\hline 8 & OCL made the system easier & 2.11 & 2.33 & 0.2612 & 0.1368 \\
\hline 9 & OCL cleared up ambiguities & 2.11 & 2.43 & 0.1645 & 0.2224 \\
\hline $10 \mathrm{C}$ & OCL helped & 1.89 & 2.19 & 0.1634 & 0.3043 \\
\hline $10 \mathrm{M}$ & OCL helped & 2.5 & 2.45 & 0.4420 & 0.5000 \\
\hline 11 & Time spent & 1.56 & 2.2 & $\mathbf{0 . 0 1 7 7}$ & $\mathbf{0 . 0 1 5 2}$ \\
\hline
\end{tabular}


Table 111. Comprehesnion-4-II and Maintenance-4-II by System

\begin{tabular}{|c|l|c|c|c|c|}
\hline & & Cab & Video & & \\
\hline 1 & & Mean & Mean & p-value (t test) & p-value (Wilcoxon) \\
\hline 2 & No problem to understand & 2.41 & 2.49 & 0.3633 & 0.0601 \\
\hline 3C & No difficulties to complete & 2.49 & 2.79 & 0.0865 & 0.1165 \\
\hline 3M & No difficulties to complete & 2.92 & 2.77 & 0.2519 & 0.2051 \\
\hline $4 \mathrm{C}$ & Hope more time & 3.64 & 3.28 & 0.0689 & 0.0682 \\
\hline $4 \mathrm{M}$ & Hope more time & 3.31 & 3.24 & 0.3917 & 0.4024 \\
\hline $5 \mathrm{C}$ & Question not easy & 2.77 & 2.51 & 0.1434 & 0.1400 \\
\hline $5 \mathrm{M}$ & Question not easy & 2.54 & 2.59 & 0.4114 & 0.4433 \\
\hline 6 & Instruction0.3014s clear & 2.1 & 1.77 & 0.0784 & $\mathbf{0 . 0 2 5 2}$ \\
\hline & & Cab & Video & & \\
\hline 7 & OCL easy to understand & 2.32 & 2.65 & 0.1507 & 0.1726 \\
\hline 8 & OCL made the system easier & 2.37 & 2.1 & 0.2189 & 0.2223 \\
\hline 9 & OCL cleared up ambiguities & 2.42 & 2.15 & 0.2021 & 0.1478 \\
\hline $10 \mathrm{C}$ & OCL helped & 2.11 & 2 & 0.3665 & 0.3048 \\
\hline $10 \mathrm{M}$ & OCL helped & 2.56 & 2.4 & 0.3247 & 0.2954 \\
\hline 11 & Time spent & 1.63 & 2.16 & $\mathbf{0 . 0 4 4 4}$ & $\mathbf{0 . 0 3 8 0}$ \\
\hline
\end{tabular}

Table 110. Comparing 2-II and 4-II

\begin{tabular}{|c|l|c|c|c|}
\hline & & Lab 2 & Lab 4 & p-value \\
\hline 1 & A lot of time to understand the system & 2.97 & 3.41 & 0.0662 \\
\hline 2 & No problems to understand & 2.68 & 2.43 & $\mathbf{0 . 0 2 0 4}$ \\
\hline 3C & No difficulties to complete Comprehension & 2.82 & 2.64 & 0.1196 \\
\hline 3M & No difficulties to complete Maintenance & 2.9 & 2.84 & 0.3576 \\
\hline 4C & Hope more time for Comprehension & 2.99 & 3.48 & $\mathbf{0 . 0 0 1 9}$ \\
\hline 4M & Hope more time for Maintenance & 3.12 & 3.31 & 0.1356 \\
\hline $5 \mathrm{C}$ & Questions not easy in Comprehension & 2.36 & 2.64 & 0.0294 \\
\hline $5 \mathrm{M}$ & Questions not easy in Maintenance & 2.43 & 2.56 & 0.1863 \\
\hline 6 & Instruction clear & 2.06 & 1.94 & 0.1474 \\
\hline
\end{tabular}

Table 112. Comparing 2-II and 4-II - attitudes of OCL

\begin{tabular}{|c|l|c|c|c|c|}
\hline & & Lab 2 & Lab 4 & p-value (t test) & p-value (Wilcoxon) \\
\hline 7 & OCL easy to understand & 2.4 & 2.48 & 0.3563 & 0.3777 \\
\hline 8 & OCL made the system easier & 2.32 & 2.23 & 0.3508 & 0.2975 \\
\hline 9 & OCL cleared up ambiguities & 2.22 & 2.28 & 0.4003 & 0.3636 \\
\hline $10 \mathrm{C}$ & OCL helped - Comprehension & 1.83 & 2.05 & 0.1445 & 0.1405 \\
\hline $10 \mathrm{M}$ & OCL helped - Maintenance & 2.59 & 2.47 & 0.3139 & 0.3308 \\
\hline 11 & Time spent & 1.62 & 2.04 & $\mathbf{0 . 0 3 5 8}$ & $\mathbf{0 . 0 3 8 5}$ \\
\hline
\end{tabular}




\section{Appendix E Assessing equal variance}

Equal variance can be assessed using the F-test [9]. As shown in Table 112, the standard deviations for both the OCL and the no_OCL subjects are provided for each task. F Ratio is the ration between corresponding standard deviations for the OCL and the no_OCL observations. $P$-value $>0.05$ indicates that equal standard deviations (variance) of the two sets of observations (OCL vs. no_OCL) cannot be rejected at $\alpha=0.05$. Table 112 shows that the assumption of equal variance holds in this experiment.

Table 112. Standard deviations and testing equal variance

\begin{tabular}{|c|c|c|c|c|c|c|c|c|c|c|c|c|c|}
\hline & & D-1 & size & $\mathrm{D}-3$ & size & $\mathrm{C}-2$ & size & C-4 & size & M-2 & size & M-4 & size \\
\hline \multirow[b]{4}{*}{ Experiment I } & OCL & 0.08 & 16 & 0.09 & 13 & 0.13 & 15 & 0.15 & 14 & 0.13 & 15 & 0.2 & 15 \\
\hline & no_OCL & 0.05 & 15 & 0.12 & 11 & 0.1 & 17 & 0.16 & 16 & 0.15 & 17 & 0.19 & 14 \\
\hline & F Ratio & \multicolumn{2}{|c|}{1.60} & \multicolumn{2}{|c|}{1.33} & \multicolumn{2}{|c|}{1.30} & \multicolumn{2}{|c|}{1.07} & \multicolumn{2}{|c|}{1.15} & \multicolumn{2}{|c|}{1.05} \\
\hline & p-value & \multicolumn{2}{|c|}{0.19} & \multicolumn{2}{|c|}{0.32} & \multicolumn{2}{|c|}{0.30} & \multicolumn{2}{|c|}{0.46} & \multicolumn{2}{|c|}{0.40} & \multicolumn{2}{|c|}{0.47} \\
\hline \multirow[b]{4}{*}{ Experiment II } & OCL & 0.06 & 40 & 0.08 & 36 & 0.13 & 39 & 0.15 & 40 & 0.17 & 39 & 0.15 & 39 \\
\hline & no_OCL & 0.07 & 36 & 0.07 & 39 & 0.14 & 40 & 0.12 & 37 & 0.13 & 40 & 0.15 & 34 \\
\hline & F Ratio & \multicolumn{2}{|c|}{1.17} & \multicolumn{2}{|c|}{1.14} & \multicolumn{2}{|c|}{1.08} & \multicolumn{2}{|c|}{1.25} & \multicolumn{2}{|c|}{1.31} & \multicolumn{2}{|c|}{1.00} \\
\hline & p-value & \multicolumn{2}{|c|}{0.32} & \multicolumn{2}{|c|}{0.35} & \multicolumn{2}{|c|}{0.41} & \multicolumn{2}{|c|}{0.25} & \multicolumn{2}{|c|}{0.20} & \multicolumn{2}{|c|}{0.50} \\
\hline
\end{tabular}

